\title{
Onordon
}

Existing Default Values and Recommendations for Exposure Assessment

A Nordic Exposure Group Project 2011 
4 norden 



\section{Existing Default Values and Recommendations for Exposure Assessment}

A Nordic Exposure Group Project 2011 
Existing Default Values and Recommendations for Exposure Assessment

A Nordic Exposure Group Project 2011

TemaNord 2012:505

ISBN 978-92-893-2316-1

http://dx.doi.org/10.6027/TN2012-505

(C) Nordic Council of Ministers

Print: Kailow Express ApS

This publication has been published with financial support by the Nordic Council of Ministers. However, the contents of this publication do not necessarily reflect the views, policies or recommendations of the Nordic Council of Ministers.

\section{www.norden.org/en/publications}

\section{Nordic co-operation}

Nordic co-operation is one of the world's most extensive forms of regional collaboration, involving Denmark, Finland, Iceland, Norway, Sweden, and Faroe Islands, Greenland, and Åland.

Nordic co-operation has firm traditions in politics, the economy, and culture. It plays an important role in European and international collaboration, and aims at creating a strong Nordic community in a strong Europe.

Nordic co-operation seeks to safeguard Nordic and regional interests and principles in the global community. Common Nordic values help the region solidify its position as one of the world's most innovative and competitive.

\section{Nordic Council of Ministers}

Ved Stranden 18

1061 København K

Telefon (+45) 33960200

\section{www.norden.org}




\section{Content}

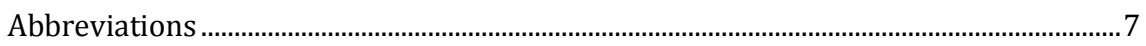

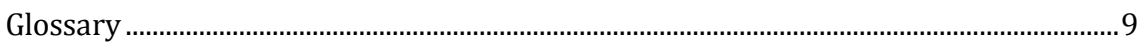

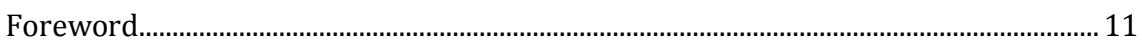

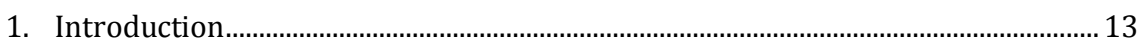

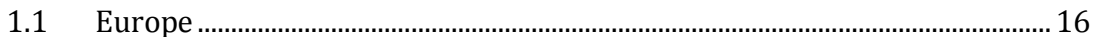

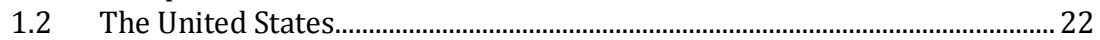

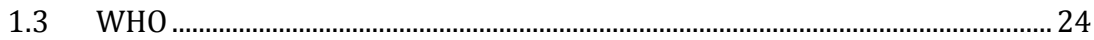

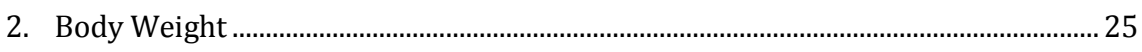

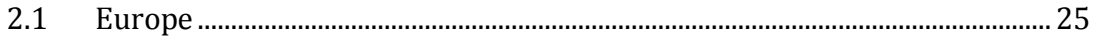

2.2 US-EPA...................................................................................................... 28

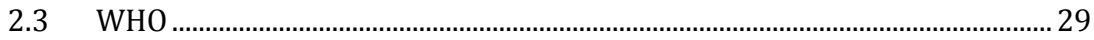

2.4 Conclusion and Recommendations................................................................ 29

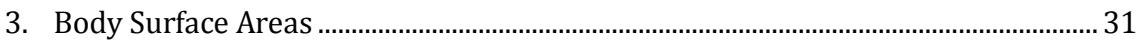

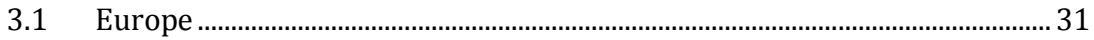

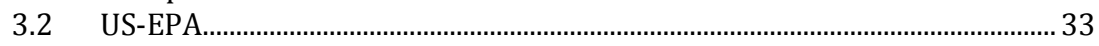

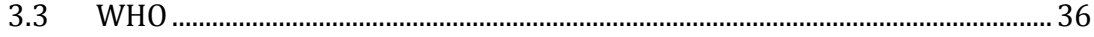

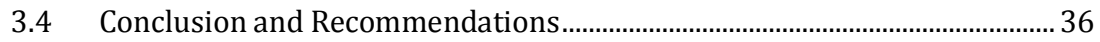

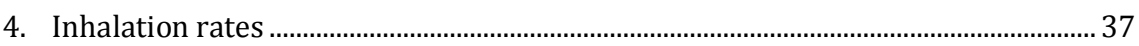

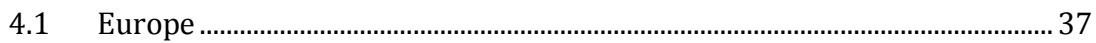

4.2 US-EPA

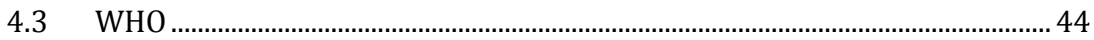

4.4 Conclusion and Recommendations.................................................................... 44

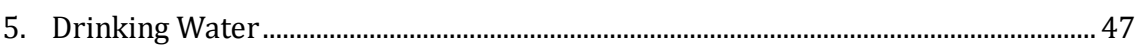

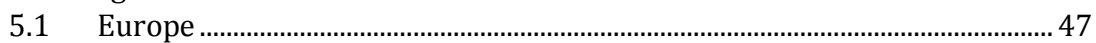

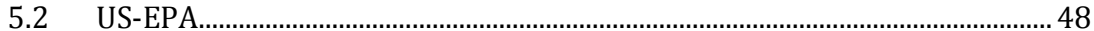

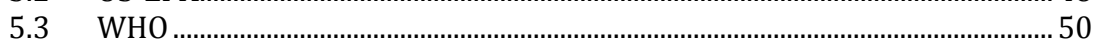

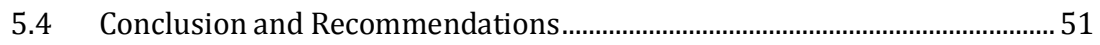

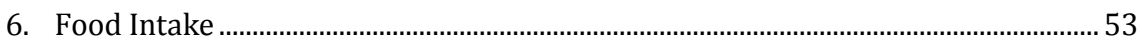

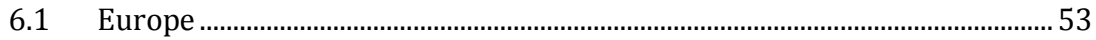

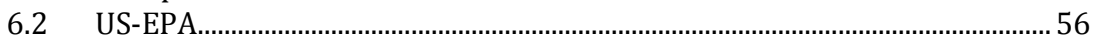

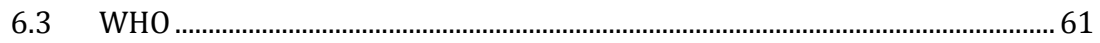

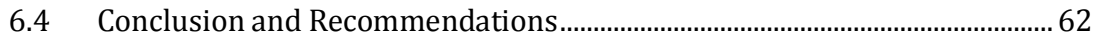

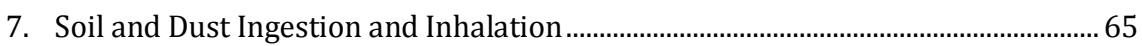

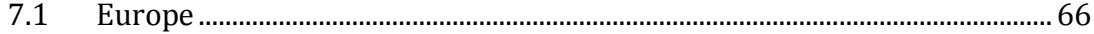

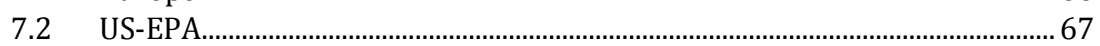

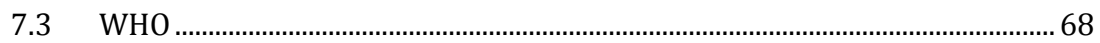

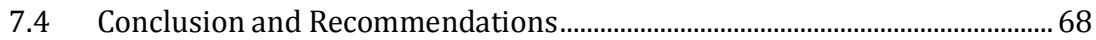

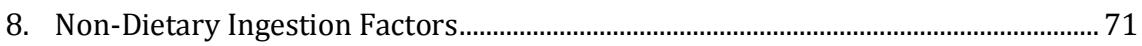

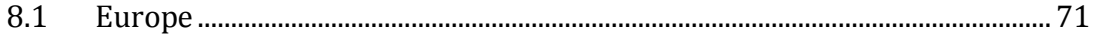

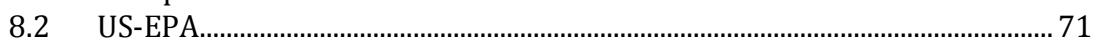

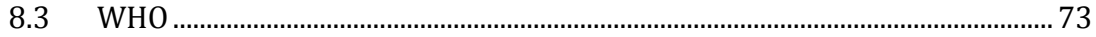

8.4 Conclusion and Recommendations................................................................... 73

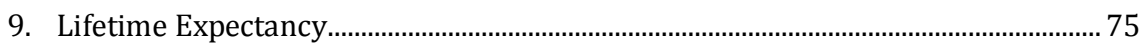

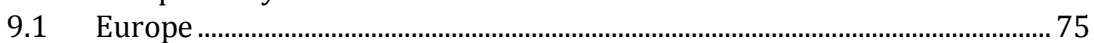

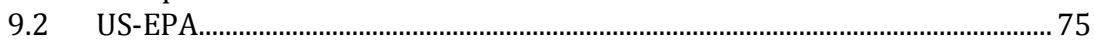

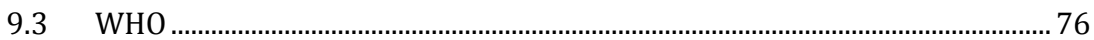

9.4 Conclusion and Recommendations................................................................ 76 


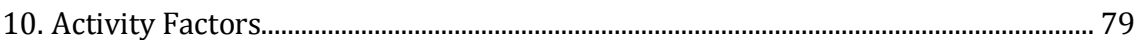

10.1 Europe

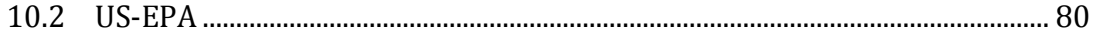

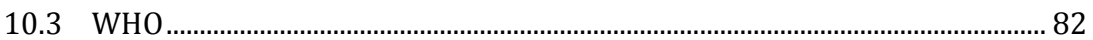

10.4 Conclusion and Recommendations................................................................... 82

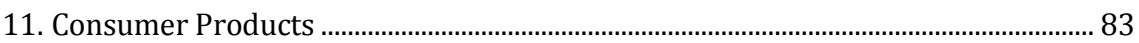

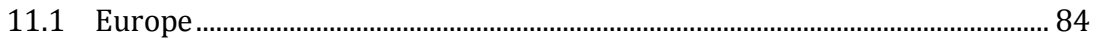

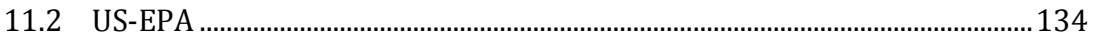

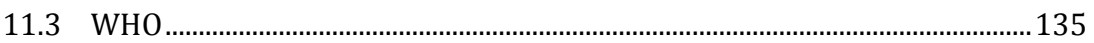

11.4 Conclusion and Recommendations...............................................................135

12. Summary, Conclusions and Recommendations......................................................137

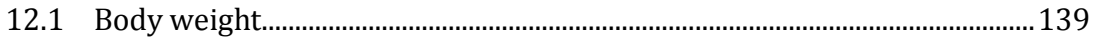

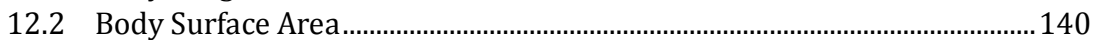

12.3 Inhalation rates............................................................................................. 140

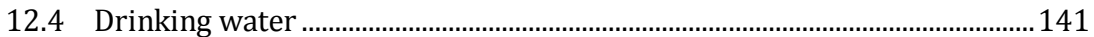

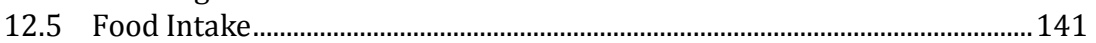

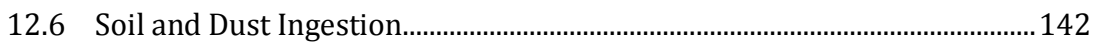

12.7 Non-Dietary Ingestion Factors ……............................................................142

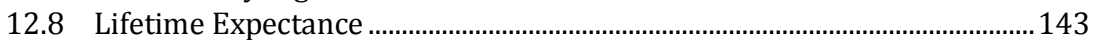

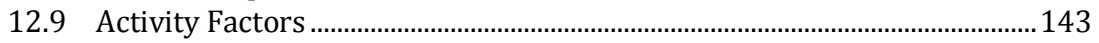

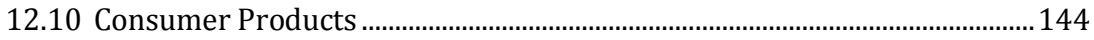

13. Annex A. Body Weight - Supplementary Tables ......................................................145

14. Annex B. Body Surface Area - Supplementary Tables ...............................................147

15. Annex C. Inhalation of air - Supplementary Tables .................................................151

16. Annex D. Drinking Water - Supplementary Tables.................................................157

17. Annex E. Food Intake - Supplementary Tables ....................................................159

18. Annex F. Soil and Dust ingestion - Supplementary Tables.....................................167

19. Annex G. Life Expectancy - Supplementary Tables..................................................169

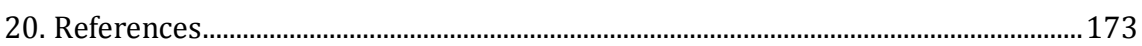




\section{Abbreviations}

\begin{tabular}{|c|c|}
\hline AlHC & American Industrial Health Council \\
\hline (C-)EFH & (Child-specific) Exposure Factors Handbook \\
\hline CEFIC-LRI & European Chemical Industry Council, Long Range Research Initiative \\
\hline CHAD & Consolidated Human Activity Database \\
\hline CSFII & Continuing Survey of Food Intake among Individuals \\
\hline D-EPA & The Danish Environmental Protection Agency \\
\hline DIY & Do-it-yourself \\
\hline ECETOC & European Centre for Ecotoxicology and Toxicology of Chemicals \\
\hline $\mathrm{ECHA}$ & European Chemicals Agency \\
\hline EFH & US-EPA Exposure Factors Handbook \\
\hline EFSA & European Food Safety Authority \\
\hline EUSES & European Union System for the Evaluation of Substances \\
\hline ExpoFacts & European Exposure Factors database \\
\hline GAF & General Assessment Factor \\
\hline ICRP & The International Commission on Radiological Protection \\
\hline IPCS & International Programme on Chemical Safety \\
\hline IRIS & Integrated Risk Information System \\
\hline METS & Metabolic equivalents \\
\hline NHANES & The National Health and Nutrition Examination Survey \\
\hline OECD & Organisation for Economic Co-operation and Development \\
\hline REACH & Registration, evaluation, authorisation and restriction of chemicals \\
\hline $\mathrm{RfC}$ & Reference Concentration \\
\hline $\mathrm{RfD}$ & Reference Dose \\
\hline RIVM & The National Institute for Public Health and Environment, Netherlands \\
\hline SCOOP & Scientific Co-operation on Questions relating to Food \\
\hline TGD & Technical Guidance Document \\
\hline TNsG & The Technical Notes for Guidance \\
\hline USDA & US Department of Agriculture \\
\hline US-EPA & The United States Environmental Protection Agency \\
\hline WHO & World Health Organization \\
\hline
\end{tabular}




\section{Glossary}

\begin{tabular}{|c|c|}
\hline Absorbed fraction & The fraction of a substance which is absorbed following exposure \\
\hline Activity factor & Factor used to characterise a given activity or time spent in a given activity \\
\hline Airborne fraction & Fraction of non-volatile material that becomes airborne in the form of droplets \\
\hline Applied dose & $\begin{array}{l}\text { Amount of a substance at the absorption barrier (skin, lung, gastrointestinal } \\
\text { tract) available for absorption }\end{array}$ \\
\hline Cloud volume & $\begin{array}{l}\text { The volume of a cloud of particles to which a person is exposed during spraying } \\
\text { of a product }\end{array}$ \\
\hline Coefficient of variation & Ratio of the standard deviation to the mean \\
\hline Confidence rating & $\begin{array}{l}\text { A qualitative rating of low, medium, or high to each recommended value in the } \\
\text { US-EPA EFH. }\end{array}$ \\
\hline ConsExpo & Computer software model for consumer exposure assessment \\
\hline Contact rate & Rate at which a product is applied to the skin, in weight per time unit \\
\hline $\begin{array}{l}\text { Contact rate aerosol } \\
\text { spray cans }\end{array}$ & The dermal contact rate during spraying of a product from an aerosol spray can \\
\hline $\begin{array}{l}\text { Contact rate trigger } \\
\text { sprays }\end{array}$ & The dermal contact rate during spraying of a product from a trigger spray \\
\hline Default value & Standard value \\
\hline Default parameter value & Standard value for a specific parameter \\
\hline Delivered dose & $\begin{array}{l}\text { The amount of a substance transported to an individual organ, tissue or fluid of } \\
\text { interest (the target) }\end{array}$ \\
\hline Density & $\begin{array}{l}\text { Measure of how much mass is contained in a given unit volume (density = } \\
\text { mass/volume) }\end{array}$ \\
\hline Dermal exposure & Direct exposure of / contact with the skin \\
\hline Diffusion & One way of transport through a membrane \\
\hline Dilution factor & Ratio of final volume/aliquot volume (final volume $=$ aliquot + diluent $)$ \\
\hline Dislodgeable amount & Amount which can be removed \\
\hline Effective (biologically) dose & Amount of a substance that actually reaches cells, sites or membranes \\
\hline $\begin{array}{l}\text { Emission Scenario Docu- } \\
\text { ment }\end{array}$ & $\begin{array}{l}\text { Document that describes the sources, production processes, pathways and use } \\
\text { patterns with the aim of quantifying the emissions (or releases) of a substance } \\
\text { into water, air, soil and/or solid waste }\end{array}$ \\
\hline Estuarine fish & $\begin{array}{l}\text { Fish living in a semi-enclosed body of water having a free connection with the } \\
\text { open sea }\end{array}$ \\
\hline Free-living & Opposite to staged activity measures \\
\hline General Fact Sheet & Fact sheet containing the general information in relation to ConsExpo \\
\hline Geometric mean & $\begin{array}{l}\text { In mathematics, a type of mean or average, which indicates the central tenden- } \\
\text { cy or typical value of a set of numbers }\end{array}$ \\
\hline Geophagy & Eating of soil \\
\hline
\end{tabular}




\begin{tabular}{|c|c|}
\hline Hazard assessment & Effect assessment, i.e. assessment of possible adverse effects of a substance \\
\hline $\begin{array}{l}\text { Inhalation cut-off diame- } \\
\text { ter }\end{array}$ & $\begin{array}{l}\text { Measure for the diameter of spray droplets that can be inhaled and reach the } \\
\text { lower areas of the lungs (alveoli, bronchioles, bronchia) }\end{array}$ \\
\hline Inhalation exposure & Direct exposure of / contact with the respiratory tract \\
\hline $\begin{array}{l}\text { Initial droplet/particle } \\
\text { distribution }\end{array}$ & The distribution of droplets/particles directly after spraying \\
\hline Internal dose & $\begin{array}{l}\text { Amount of a substance that has been absorbed and is available for interaction } \\
\text { with the biologically target organs and tissues }\end{array}$ \\
\hline Key study & In the US-EPA EFH, the most useful studies for deriving exposure factors \\
\hline Leachable fraction & Relative amount of chemical which can leach from a product \\
\hline Lognormal distribution & $\begin{array}{l}\text { Probability distribution of a random variable whose logarithm is normally } \\
\text { distributed }\end{array}$ \\
\hline $\begin{array}{l}\text { Lognormal body weight } \\
\text { distribution }\end{array}$ & $\begin{array}{l}\text { Probability distribution of body weight variables whose logarithm are normally } \\
\text { distributed }\end{array}$ \\
\hline Mass generation rate & $\begin{array}{l}\text { Mean mass generation rate during the entire duration of spraying calculated } \\
\text { from the sprayed amount and the total duration of spraying }\end{array}$ \\
\hline Molecular Weight Matrix & Parameter used to calculate the relative vapour pressure of a component \\
\hline Mouthing times & Duration of an object kept in the mouth \\
\hline Neonatal weight & Body weight of a new-born infant \\
\hline Oral exposure & Direct exposure of / contact with the gastro-intestinal tract \\
\hline Per capita & Per person \\
\hline Physiologic information & Information on physiological parameters \\
\hline Pica & Deliberate ingestion of larger amounts of non-nutritive substances \\
\hline Planimeter & $\begin{array}{l}\text { Measuring instrument used to determine the area of an arbitrary two- } \\
\text { dimensional shape }\end{array}$ \\
\hline Pneumatic & Mechanism activated, controlled, or powered by air or gas pressure \\
\hline Postpartum & Period just after delivery \\
\hline Potential dose & $\begin{array}{l}\text { Amount of a substance contained in material ingested, inhaled or in material } \\
\text { applied to the skin }\end{array}$ \\
\hline Product amount & Amount of a product used in a specific use scenario \\
\hline $\begin{array}{l}\text { Recreational marine } \\
\text { intake }\end{array}$ & $\begin{array}{l}\text { Consumption of marine fish catch as part of a sporting or recreational activity } \\
\text { and not for the purpose of providing a primary source of food }\end{array}$ \\
\hline Relevant study & $\begin{array}{l}\text { In the US-EPA EFH, studies that are applicable or pertinent, but not necessarily } \\
\text { the most important to derive exposure factors }\end{array}$ \\
\hline Spray duration & Duration of spraying \\
\hline Total duration & Entire duration of an activity \\
\hline Transfer coefficient & $\begin{array}{l}\text { The "transfer coefficient" is a quantification of the transfer of powder from the } \\
\text { carpet surface to human skin per unit time as e.g. a child is crawling across a } \\
\text { treated carpet, wiping some of the powder }\end{array}$ \\
\hline Use duration & Duration related to the use of a product \\
\hline Weight fraction & $\begin{array}{l}\text { Weight fraction of a solution/dilution is the weight fraction of the product } \\
\text { divided by the dilution factor }\end{array}$ \\
\hline
\end{tabular}




\section{Foreword}

The project was funded by the Nordic Council of Ministries through the Nordic Chemicals Group. The project was launched and coordinated by the Nordic Exposure Group, which also act as a project steering group. The practical work was done two consultants from Division of Toxicology and Risk Assessment National Food Institute, Technical University of Denmark

The draft version of the report was presented in the Nordic Workshop, on $3^{\text {th }}-4^{\text {th }}$ of May 2011 in Oslo. The report was finalised based on the major comments from the WS participants.

The members of the steering group and consultants are introduced below:

Project steering group (the Nordic Exposure Group)

\begin{tabular}{ll}
\hline Member & Country \\
\hline $\begin{array}{l}\text { Lena Höglund } \\
\text { Jouni Räisänen }\end{array}$ & $\begin{array}{l}\text { Danish Environmental Protection Agency, Denmark } \\
\text { Safety and Chemicals Agency, Finland } \\
\text { Anna-Maija Hämäläinen } \\
\text { Margareta Warholm } \\
\text { Marianne van der Hagen } \\
\text { Abdulqadir Suleiman }\end{array}$ \\
$\begin{array}{l}\text { Swedish Chemicals Agency, Finland } \\
\text { Climatr Kristjánsson and Pollution Agency, Norway }\end{array}$ \\
\hline & $\begin{array}{l}\text { Arbeidstilsynet, Norway } \\
\text { Administration of Occupational, Safety and Health, Iceland }\end{array}$ \\
Consultants & \\
\hline Names & \\
\hline Elsa Nielsen and & Organisation \\
Tine Iskov Kopp & Division of Toxicology and Risk Assessment \\
\end{tabular}




\section{Introduction}

The International Programme on Chemical Safety (IPCS) has defined exposure as the "contact of an organism with a chemical or physical agent, quantified as the amount of chemical available at the exchange boundaries of the organism and available for absorption." (WHO/IPCS, 2001). This means contact with the visible exterior of a person such as the skin, and openings such as the mouth, nostrils, and lesions.

The process of a chemical entering the body can be described in two steps: Contact (exposure) followed by entry (crossing the boundary, i.e., absorption). The process begins with a chemical being released from a source into the environment. Once in the environment, the chemical can be transformed and transported through the environment via air, water, soil, dust, and food. Individuals become in contact with the chemical in the environment through inhalation, ingestion, or skin/eye contact. Individuals may also come in contact with chemicals in consumer products as well as during work. The individual's activity patterns as well as the concentration of the chemical will determine the magnitude, frequency, and duration of the exposure. The exposure becomes an absorbed dose when the chemical crosses an absorption barrier (entry into the body). When the chemical or its metabolites interact with a target tissue, it becomes a target tissue dose, which may lead to an adverse health outcome, the biologically effective dose. Figure 1 illustrates the relationship between exposure and different types of dose.

Figure 1. Correlation between exposure concentration and internal dose. Modified from US-EPA (1997)

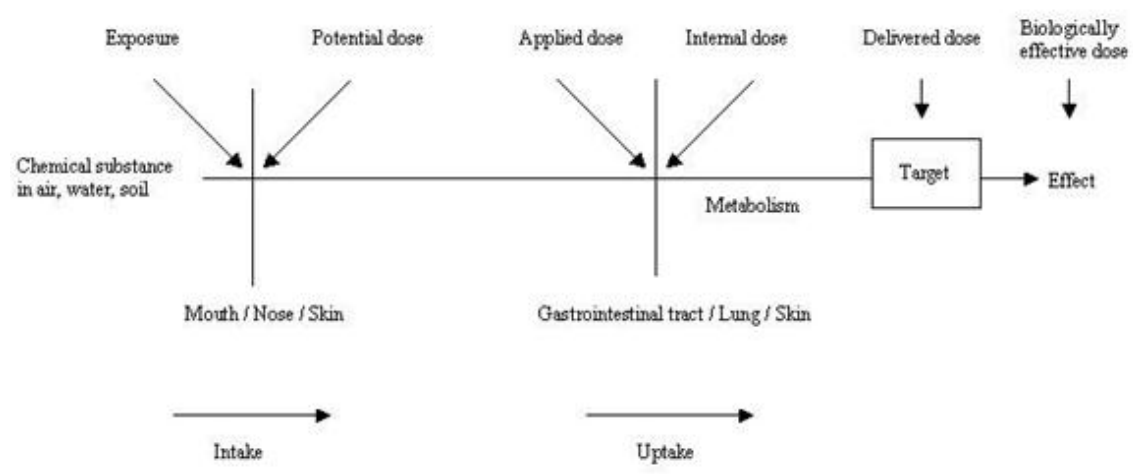

The applied dose is the amount of a substance at the absorption barrier (skin, lung, gastrointestinal tract) available for absorption. Usually it is very difficult to measure the applied dose directly at the absorption barrier. An approximation of the applied dose can be made using the poten- 
tial dose, which is the amount of the substance ingested, inhaled or in material applied to the skin. The applied dose, or the amount that reaches the exchange boundaries of the skin, lung or gastrointestinal tract, may often be less than the potential dose if the substance is only partly bioavailable. The amount of a substance that has been absorbed and is available for interaction with the biologically target organs and tissues is called the internal dose. The amount transported to an individual organ, tissue or fluid of interest (the target) is termed the delivered dose (or target tissue dose); the delivered dose may be only a part of the total internal dose. The biologically effective dose, or the amount that actually reaches cells, sites or membranes where adverse effects occur, may only be a part of the delivered dose. Doses are often presented as dose rates, or the amount of a chemical dose (applied or internal) per unit time (e.g., $\mathrm{mg} /$ day), or per-unit-body-weight-basis (e.g., $\mathrm{mg} / \mathrm{kg}$ body weight per day). (WHO/IPCS, 1999).

According to the REACH "Guidance on Information Requirements and Chemical Safety Assessment" published by the European Chemicals Agency (ECHA) (ECHA, 2010), "exposure to a particular substance should normally be understood as meaning external exposure. This can be defined as the amount of the substance ingested, the amount in contact with the skin, and/or the amount inhaled (which is represented by the airborne concentration of the substance in the breathing zone). It does not usually refer to concentrations within the body, which are determined by the amount of the substance absorbed from the digestive system, respiratory system, or entering the body through the skin." Exposure can be considered as a single event, as a series of repeated events or as continuous exposure.

The aim of an exposure assessment is to determine the nature and extent of contact with chemical substances experienced or anticipated under different conditions. An exposure assessment is the quantitative or qualitative evaluation of the amount of a substance that humans come into contact with and includes consideration of the intensity, frequency and duration of contact, the route of exposure (e.g., dermal, oral or respiratory), rates (chemical intake or uptake rates), the resulting amount that actually crosses the boundary (a dose), and the amount absorbed (internal dose). Depending on the purpose of an exposure assessment, the numerical output may be an estimate of the intensity, rate, duration and frequency of contact exposure or dose (the resulting amount that actually crosses the boundary). (WHO/IPCS, 1999).

Exposure data can be either measured or calculated. Measured exposure data are preferred, if they are valid. Often measured exposure data are not available, or they are not considered valid, and therefore modelgenerated data must be used. An exposure model is "a conceptual or mathematical representation of the exposure process" (WHO/IPCS, 2004); i.e., a tool to calculate an estimate (a figure) to use in the risk characterisation, e.g., where the outcome of the hazard assessment is compared with 
an exposure estimate. The output of an exposure model can be an exposure concentration; in practice, however, exposure often includes estimates of intake (e.g., amount of chemical inhaled or ingested) and the amount of a chemical that is absorbed into the body (e.g., amount of chemical that penetrates the skin or the lining of the lung) (WHO/IPCS, 2005). Exposure models can be developed to estimate exposures and doses of individuals, defined population groups, or entire populations. Exposure may be estimated as a continuous variable or integrated over time ranging from minutes to a lifetime. The modelled outputs may include mean or median values, distribution parameters (standard deviations, quartiles, ranges), or entire distributions. Consequently, exposure models vary widely in complexity, approach, inputs, and outputs (WHO/IPCS, 2005).

In order to perform an exposure assessment, it is necessary to apply various "non-chemical-specific" exposure related parameters such as e.g., body weight, body surface area, activity factors, ventilation rates, ingestion of water/food etc. These parameters are called non-chemicalspecific exposure factors and are generally drawn from the scientific literature or governmental statistics.

The approach to exposure assessment is not as internationally harmonised as hazard assessment. Although broad consistency in the overall approaches used by different bodies and countries in conducting exposure assessment exists, there is variation in the types of approaches and tools used, including the use of exposure factors.

The purpose of this report is to give an overview of non-chemicalspecific exposure factors to be used by the authorities during the process of assessing exposure to both adults and children as well as of risk assessment in relation to REACH, and to contribute towards a further harmonisation of such exposure factors to be used in exposure assessments. Thus, the process of exposure assessment in itself is not further addressed in this report. . The percentile chosen is a decision that has to be made by the assessor depending on the purpose of the assessment. For transparency, the reasoning for the choice of values should always be available in the assessment report.

The following non-chemical-specific exposure factors are addressed in this report:

- Body weight

- Body surface areas

- Inhalation rates

- Ingestion of drinking water

- Intake of food

- Ingestion of soil and dust

- Non-dietary ingestion factors

- Lifetime expectancy

- Activity factors

- Consumer products 
In the following, guidance and recommendations provided by various EU bodies, with the main focus on the information gathered in the REACH "Guidance on Information Requirements and Chemical Safety Assessment" (REACH GD IR\&CSA) published by the European Chemicals Agency (ECHA) (ECHA, 2010), will be addressed first as this report is directed primarily towards the exposure factors to be used in exposure assessments in the context of the EU chemical regulation "REACH". Then the US guidance and recommendations will be addressed using US-EPA Exposure Factor Handbook, at the time (December 2010) available in the form of an external review draft (US-EPA, 2009), which provides the most comprehensive overview and data basis, considerations, evaluations and recommendations in the area of non-chemical-specific exposure factors. The revised Exposure Factor Handbook was published in September 2011, after the finalisation of the work in this Nordic report. Finally, guidance and recommendations provided by the WHO will be addressed as this report also is meant to contribute towards a further harmonisation of exposure factors to be used in exposure assessments.

Below, the main EU, US and WHO sources of non-chemical-specific exposure factors will be addressed. The OECD has not published any guidance or recommendations on the use of non-chemical-specific exposure factors.

\subsection{Europe}

\subsubsection{ECHA}

The European Chemicals Agency (ECHA) under REACH has published a "Guidance on Information Requirements and Chemical Safety Assessment" (REACH TGD) that describes the information requirements under REACH with regard to substance properties, exposure, use and risk management measures, and the chemical safety assessment (ECHA, 2010). It is part of a series of guidance documents that are aimed to help all stakeholders with their preparation for fulfilling their obligations under the REACH regulation. These documents cover detailed guidance for a range of essential REACH processes as well as for some specific scientific and/or technical methods that industry or authorities need to make use of under REACH.

Part D of the REACH TGD details how to develop exposure scenarios and related exposure estimation. Part D provides also links to more indepth guidance on exposure assessment. This includes: Occupational exposure estimation (Chapter R.14), exposure estimation related to consumers (Chapter R.15), and exposure estimation related to the environment (Chapter R.16). Recommendations on exposure factors are generally limited although Chapter R.15 provides some information regarding default values for exposure factors to be used for consumer exposure 
assessment, such as amount of product used per application and exposure time. These recommendations are generally obtained from the RIVM (The National Institute for Public Health and the Environment, Netherlands) fact sheets for specific products, in order to build consistency with ConsExpo, a well-known computer tool for consumer exposure assessment (see Section 1.1.2). Chapter R.16 provides some information regarding default values to be used for exposure factors related to the environment, such as human intake rates of crops, meat and fish, dairy products and drinking water; these default values are used in EUSES, the computer tool used for exposure assessment related to the environment (see Section 1.1.3).

Chapter R8, Table R. 8-18, of the REACH TGD "Characterisation of dose [concentration]-response for human health" (ECHA, 2008a) also provides some information regarding default values for exposure factors to be used for exposure assessments of consumers and man exposed indirectly via the environment as well as for exposure assessments of workers. It should be noted that the recommendations in Table R.8-18 are stated as "taken from Gold et al., 1984 and ICRP, 1975"; however, it is not clear whether the recommendations are taken from one of these references or both. The reference "ICRP, 1975" is to the International Commission on Radiological Protection (ICRP) Publication No. 23 "Report of the Task Group on Reference Man", which was adopted in October 1974 and published in April 1975. It should be noted that other bodies, e.g., WHO (WHO/IPCS, 1994 and 1999) refer to this ICRP report as "ICRP, 1974."

\subsubsection{ConsExpo}

The National Institute for Public Health and the Environment, Netherlands (RIVM) is a Dutch research institute that is an independent agency of the Dutch Ministry of Health, Welfare and Sport. In order to mathematically predict human exposure to consumer products, RIVM has developed the software model ConsExpo (RIVM, 2010). ConsExpo, which can be downloaded from the internet (the link can be find in the reference list, reference "RIVM, 2010"), is a set of coherent, general models that enables the estimation and assessment of the exposure to substances from consumer products and their uptake by humans. ConsExpo models and ConsExpo data can be used, where appropriate, in order to assess also professional exposure. The assessor should though take the differences between professional and non-professional uses (e.g. use period, use frequency or use of PPEs) into consideration (HEEG (Human Exposure Expert Group) opinion, endorsed on the second Technical Meeting on Biocides 2008). Data about the application of products and data from mathematical models are used to build up the program. The program is based on relatively simple exposure and uptake models. The starting point for these models is the route of exposure, i.e. the inhalation, dermal or oral route. The most appropriate exposure scenario and 
uptake model is chosen for each route. The parameters needed for the exposure scenario and the uptake models are then filled in. It is possible that exposure and uptake occur simultaneously via different routes. In addition to data about the exposure and uptake, general scenario data is also needed, such as the frequency and body weight of the exposed person. Using the data mentioned above, ConsExpo calculates the exposure and uptake. For this purpose fact sheets are developed.

The fact sheets give information that is important for the consistent estimation and assessment of the exposure to, and the uptake of, substances from consumer products, such as paint, do-it-yourself products, cosmetics and cleaning products. In the fact sheets, information about exposure to chemical substances is bundled into certain product or exposure categories. These categories are chosen so that products with similar exposures can be combined. On the one hand, the fact sheet gives general background information, while on the other hand, it quantifies exposure parameters which, together with an exposure scenario, or a combination of the various exposure scenarios, produces a quantitative estimate of the exposure. (RIVM, 2010).

In the General Fact Sheet, (RIVM, 2006a) general information is presented that is needed for consumer exposure assessment in addition to the more specific information provided in the fact sheets on the different product groups. Information includes: The volume and surface area of rooms in Dutch dwellings, the air-change rate in various rooms in dwellings and the total body surface and surface of body parts of adults and children. Also, general information on the fact sheets is given, such as a description the format of the fact sheets, the connection with the ConsExpo program and the boundary conditions under which the defaults in the fact sheet are defined.

In addition to the General Fact Sheet, fact sheets are available for children's toys (RIVM, 2002), cleaning products (RIVM, 2006c), cosmetics (RIVM, 2006b), disinfectants (RIVM, 2006d), "do-it-yourself products" (RIVM, 2007a), paints products (RIVM, 2007b) and pest control products (RIVM, 2006e).

In the Exposure to chemicals via house dust, (RIVM, 2008), the potential of house dust being a health risk is investigated and in this connection, exposure to dust via ingestion and inhalation have been examined.

The consumer exposure estimation should normally address the intended uses of the products that contain the substances under investigation. However, since consumers may not accurately follow instructions for use of products, an estimation of other reasonably anticipated uses should be made (ECHA, 2008b).

In the fact sheets provided by the RIVM, the basis for the calculation and/or estimation of the default parameter values is a realistic worstcase scenario, and considers consumers who frequently use a certain product under relatively less favourable circumstances. For example, when using a cosmetic product, basic assumptions are: relatively fre- 
quent use, application of a relatively large amount in a small room with a low ventilation rate, and a relatively long stay in that room. The parameter values in the fact sheets are aimed at (Dutch) consumers. They are chosen such that a relatively high exposure and uptake are calculated, in the order of magnitude of a 99th percentile of the distribution. To achieve this goal, the $75^{\text {th }}$ or the $25^{\text {th }}$ percentile is calculated (or estimated) for each parameter. The $75^{\text {th }}$ percentile is used for parameters which give a higher exposure for higher values, and the $25^{\text {th }}$ percentile is used in the reverse case (RIVM, 2006a).

\subsubsection{EUSES}

The European Union System for the Evaluation of Substances (EUSES) is a decision-support instrument, which enables government authorities, research institutes and chemical companies to carry out rapid and efficient exposure assessments for chemical substances (ECB, 2010).

Indirect exposure of humans via the environment may occur by consumption of food (fish, crops, meat and milk) and drinking water, inhalation of ambient air, and ingestion of soil. For existing substance, measured levels in various environmental compartments may be available; however, for new substances, usually no relevant measured data are available and concentrations of a substance in the environment must be estimated.

The indirect exposure is estimated by the use of EUSES. EUSES estimates concentrations in food and the total daily intake of a substance based on predicted environmental concentrations for (surface) water, groundwater, soil, sediment and ambient air. The indirect exposure is principally assessed on two spatial scales: locally near a point source of the substance, and regionally using averaged concentrations over a larger area. A third spatial scale, the continental scale, is also assessed by EUSES; however, this scale is not included in the estimations of the indirect exposure.

EUSES is intended mainly for the initial (screening) and intermediate (refined) stages of exposure assessments rather than comprehensive assessments. On the basis of the screening, it can be decided whether more data need to be generated and whether a more refined assessment is necessary. EUSES can also be applied for refined assessments by allowing the replacement of default values, estimated parameter values, or intermediate results by more accurately estimated values or by measured data. EUSES is not specifically designed for site-specific assessments (defaults represent a standard region in EU), but adjustment of parameters may allow for insight into specific local or regional situations.

The system is fully described in the extensive EUSES documentation and is based on the EU Technical Guidance Documents (TGD) on Risk Assessment for New Notified Substances, Existing Substances and Biocides (EC, 2003). The new EUSES 2.1 version (2008) is an update of EUSES 2.0, containing all Emission Scenario Documents for biocides. The 
development of EUSES 2.1 was commissioned by the European Commission to the National Institute of Public Health and the Environment (RIVM) of The Netherlands. The documentation and the program can be downloaded (free of charge) from the Ex-ECB Website (ECB, 2010).

\subsubsection{TNsG}

According to the Biocidal Products Directive, a risk assessment of biocidal products is required before these can be placed on the European Market. The estimation of human exposure is fundamental for the risk assessment and requires quantification of the levels of exposure for both users of the biocidal product and others who may be exposed following its use. As there is still a paucity of exposure data on biocides, various approaches are used to estimate human exposure to them. From 1998 onwards the European Union funded a series of projects to both fill this knowledge gap and establish a harmonised approach for assessing human exposure to biocides. The outcome of these projects was the publication of the Technical Notes for Guidance on Human Exposure to Biocidal Products (TNsG). (TNsG, 2007).

The most recent version of the TNsG (TNsG, 2007) covers all product types and presents worked examples for each of them. This TNsG consists of a written part, as well as a computerised database (BEAT) of exposure data (largely for occupational settings), and the consumer exposure model ConsExpo. The worked examples are indicated in the written part and are described in detail in the database.

\subsubsection{ExpoFacts}

In 2002, the European Exposure Factors (ExpoFacts) database started as a two-year project funded by CEFIC-LRI (European Chemical Industry Council, Long Range Research Initiative) to create a European database of factors affecting exposure to environmental contaminants. The aim was to create a public access data source, similar to the US-EPA Exposure Factors Handbook, 1997, which has been widely used by European researchers, but with European data. Since 2007, the project is hosted by the European Commission's Joint Research Centre. (JRC, 2010).

When the project was started in 2002, European exposure factor data were scattered within numerous national and international institutions. The ExpoFacts database has created no new data, but instead compiled the existing data into one Internet database, where it can be easily found, screened and downloaded from. Data were collected from the EU 
countries, candidate countries to EU, and EFTA ${ }^{1}$ countries. As a result, the ExpoFacts database contains data from 30 European countries. In addition to the population time use patterns and exposure route information, e.g., dietary statistics, the database contains socio-demographic and physiologic information to enable database use as a tool for population-wide exposure modelling and risk assessment. (JRC, 2010).

The methodological information, which is provided in the ECETOC sourcebook (ECETOC, 2001), is not found in the ExpoFacts database. Therefore, the ExpoFacts database can not replace the ECETOC sourcebook, but adds new information and Internet accessibility. (JRC, 2010).

As the ExpoFacts database has compiled the existing data, but created no new data, this database has not been used as a primary source for exposure factors in this report.

\subsubsection{ECETOC Sourcebook}

ECETOC (European Centre for Ecotoxicology and Toxicology of Chemicals) has published a report with European exposure factors titled " $E x$ posure Factors Sourcebook for European Populations, with Focus on UK Data" (ECETOC, 2001). This document was the first broader compilation of European exposure factors and contains methodological information. The ECETOC sourcebook updates and builds upon other collections of exposure factor data including the 1994 American Industrial Health Council's Exposure Factors Sourcebook (AIHC) and the US-EPA Exposure Factors Handbook (US-EPA, 1997); the AIHC no longer exists. The information in the ECETOC sourcebook includes physiological parameters (body weight, body surface areas, life expectancy), time-activity patterns (work hours, indoor/outdoor time, etc.), and receptor contact rates (soil ingestion rates, food consumption rates, etc.). The ECETOC sourcebook is not publicly available.

\subsubsection{Other}

In addition to the above-mentioned sources of exposure factors, there are a number of other sources. These sources will not be described here but will appear in the following sections when appropriate.

\footnotetext{
${ }^{1}$ European Free Trade Association, presently comprising Iceland, Liechtenstein, Norway and Switzerland. European countries, that are not EU member states, can cooperate with EU via EFTA.
} 


\subsection{The United States}

The US-EPA Exposure Factors Handbook (EFH) (US-EPA, 2009) has been prepared to provide information and recommendations on various factors used in assessing exposure to both adults and children. The handbook provides non-chemical-specific data on a number of physiological and behavioural exposure factors such as e.g., body weight, dermal factors, life time, activity factors, inhalation rates, ingestion of water, food items and soil/dust, consumer products.

The EFH was first published in 1989, revised in 1997, and then in 2009. The 2009 version is currently (October 2010) available in the form of an external review draft (US-EPA, 2009). Recognizing that exposures among infants, toddlers, adolescents, and teenagers can vary significantly, the US-EPA published the Child-Specific Exposure Factors Handbook (C-EFH) in 2002 and its revision in 2008 (US-EPA, 2008). The 2009 version of the EFH incorporates new factors and data provided in the 2008 version of the C-EFH as well as other relevant information published through June 2009.

In order to promote scientific consensus on risk assessment issues, US-EPA has developed the Guidance for Selecting Age Groups for Monitoring and Assessing Childhood Exposures to Environmental Contaminants (US-EPA, 2005). This guidance document recommends a set of age groupings based on current understanding of differences in life stage behaviour and anatomy and physiology that can serve as a starting set for consideration by risk assessors and researchers. The 2008 version of the C-EFH as well as the 2009 version of the EFH reflect the age categories recommended in this guidance document: Birth to $<1$ month, 1 to $<3$ months, 3 to $<6$ months, 6 to $<12$ months, 1 to $<2$ years, 2 to $<3$ years, 3 to $<6$ years, 6 to $<11$ years, 11 to $<16$ years, 16 to $<21$ years. Adult data (i.e., for individuals $>21$ years old), on the other hand, are presented using the age groups defined by the authors of the individual studies.

The US-EPA Consolidated Human Activity Database (CHAD) (US-EPA, 2007a) contains data obtained from pre-existing human activity studies that were collected at city, state, and national levels. CHAD is intended to be an input file for exposure/intake dose modelling and/or statistical analysis. CHAD is a master database providing access to other human activity databases using a consistent format. This facilitates access and retrieval of activity/and questionnaire information from those databases that US-EPA currently has access to and uses in its various regulatory analyses undertaken by program offices. CHAD provides no recommendations on human activity, but their data are used in order to calculate recommendations by other studies cited in this report.

The data presented in the EFH (US-EPA, 2009) have been compiled from various sources, including government reports and information presented in the scientific literature. The studies presented in the EFH were chosen because they were considered by the US-EPA as being use- 
ful and appropriate for recommending exposure factors. When evaluating the scientific and technical information, the US-EPA has applied five so-called "general assessment factors" (GAFs):

- Soundness (adequacy of approach and minimal or defined bias)

- Applicability and utility (focus on the exposure factor of interest, representativeness of the population, currency of the information, and adequacy of the data collection period)

- Clarity and completeness (accessibility, reproducibility, and quality assurance)

- Variability and uncertainty (variability in the population and uncertainty in the results)

- Evaluation and review (level of peer review and number and agreement of studies)

These GAFs were adapted and expanded to include specific considerations deemed to be important during evaluation of exposure factors data, and were used to judge the quality of the underlying data used to derive recommendations.

The studies included in the EFH (US-EPA, 2009) were designated as "key studies" or "relevant studies." Key studies were generally defined as the most useful for deriving recommendations for exposure factors. The recommended values for most exposure factors in the EFH are based on the results of such key studies. Other studies were designated as "relevant studies" meaning that they provided applicable or pertinent data, but not necessarily the most important ones for a variety of reasons (e.g., data were outdated, limitations in study design). The distinction between key and relevant studies was on the GAFs. This report include predominantly the studies designated as "key studies" and only a few of the studies designated as "relevant studies" have been selected for inclusion.

The US-EPA (US-EPA, 2009) has assigned a confidence rating of low, medium, or high to each recommended value (US-EPA, 2009). It has been underscored that this qualitative rating was not intended to represent uncertainty analyses but to represent the US-EPA's judgment on the quality of the underlying data used to derive the recommendations. The judgment was made using the GAFs. It is noted that there is a continuum from low to high, and that judgment was used to assign a rating to each factor. The recommendations given in the EFH (US-EPA, 2009) are accompanied by a discussion of the rationale for their rating.

In the providing of recommendations for the various exposure factors, the US-EPA has made an attempt to present percentile values that are consistent with the exposure estimators defined in Guidelines for Exposure Assessment (US-EPA, 1992), i.e., mean and upper percentile (US-EPA, 2009). It is noted that this was, however, not always possible, because the available data were limited for some exposure factors, or the authors of the study did not provide such information. In the EFH 
(US-EPA, 2009), the term "upper percentile" is intended to represent values in the upper tail, i.e., between $90^{\text {th }}$ and $99.9^{\text {th }}$ percentile of the distribution of values for a particular exposure factor.

Finally, it is underscored that the recommendations provided in the EFH (US-EPA, 2009) should be interpreted as suggestions that exposure/risk assessors can consider and modify as needed based on their own evaluation of a given risk-assessment situation.

\subsection{WHO}

The WHO/IPCS has undertaken a project to harmonize approaches to the assessment of risk from exposure to chemicals through increased understanding. The project focuses on specific issues and attempts to achieve agreement on basic principles. Among the project's focus areas are exposure assessment and terminology for exposure assessment and risk assessment. The status for the activities of the harmonization project is published in a newsletter. (WHO/IPCS, 2010).

Under this project, an IPCS Harmonization Project Document on the Principles of Characterizing and Applying Human Exposure (WHO/IPCS, 2005) and an IPCS Harmonization Project Document on Characterizing and Communicating Uncertainty in Exposure Assessment (WHO/IPCS, 2008) have been published. None of these documents contain specific recommendations on default values for human exposure factors.

The WHO recommendations on exposure factors included in this report stem mainly from data published by the International Commission on Radiological Protection (ICRP, 1974). These recommendations are to be found in reports which are produced in cooperation with the IPCS, namely the Environmental Health Criteria 170 and 210 (WHO/IPCS, 1994 and 1999 respectively). The reference "ICRP, 1974" is to the ICRP Publication No. 23 "Report of the Task Group on Reference Man", which was adopted in October 1974 and published in April 1975. It should be noted that ECHA, in the REACH TGD (ECHA, 2010), refers to this ICRP report as "ICRP, 1975".

The WHO/IPCS Environmental Health Criteria 214 (WHO/IPCS, 2000) addresses human exposure assessment in general and a section on exposure factors are included; however, no recommendations on specific values are provided in this document. 


\section{Body Weight}

The average daily dose is a dose that is typically normalized to the average body weight of the exposed population. If exposure occurs only during childhood years, the average child body weight during the exposure period should be used to estimate risk. Conversely, if adult exposures are being evaluated, an adult body weight value should be used. (USEPA, 2009).

\subsection{Europe}

In the REACH Guidance on information requirements and chemical safety assessment Chapter R8 (ECHA, 2008a), a body weight of $70 \mathrm{~kg}$ (men and women combined) is recommended for exposure assessments of consumers and man exposed indirectly via the environment as well as for workers (Table R. 8-18).

In the REACH Guidance on information requirements and chemical safety assessment Chapter R15: Consumer exposure estimation (ECHA, 2008b), it is stated that default body weights of $70 \mathrm{~kg}$ for adult males and $60 \mathrm{~kg}$ for adult females may in principle be used. For further analyses, particularly for estimations of children's exposure, it is stated that more detailed compilations of body weights (including distributions) are available for Germany (AUH, 1995), The Netherlands (Bremmer et al., 2006; please note that this reference is to the ConsExpo General Fact Sheet RIVM, 2006a), as well as for the US (e.g., US-EPA, 1997), but no further recommendations are provided.

In the Preparation of a Guidance Document on Pesticide Exposure; Assessment for Workers, Operators, Bystanders and Residents (EFSA, 2010), it is proposed that the body weight of an adult should be taken as $60 \mathrm{~kg}$, and those for children aged 10 to $<12$ months, 1 to $<3$ years, 3 to $<6$ years, 6 to $<11$ years, and 11 to $<16$ years, as 8.7, 12.3, 17.5, 28.7 and $50.2 \mathrm{~kg}$ respectively. EFSA has cited ECETOC (2001) as the reference, see below. On request from EFSA the Scientific Committee has recently developed a guidance document on harmonisation of default values used by EFSA's Scientific Panels, Committees and Units (Draft guidance on Default assumptions 2011). The Scientific Committee recommends a body weight of $70 \mathrm{~kg}$ as default for European adults. For dietary exposure assessment, a body weight of $12 \mathrm{~kg}$ should be used as default for European infants and children. If deviation from the default value is required for the assessment of specific age groups, the median values identified in Table 2.1 should be used. 
According to the Report from the Commission on Dietary Food Additive Intake in the European Union, Commission of the European Communities (2001), the default value for young children (under three years), which is 15 $\mathrm{kg}$ of body weight and the default value for adults, which is $60 \mathrm{~kg}$, should be used when reporting monitored data. This report is the outcome of the SCOOP Task 4.2 on "Methodologies for monitoring of food additive intakes."

RIVM has calculated default values on body weight for children of various age groups, presented in Annex B, Tables B.2 and B.3. For adult men, a body weight of $74 \mathrm{~kg}$ is recommended and for adult women a weight of $61 \mathrm{~kg}$ is recommended. For both men and women, RIVM has provided a default value of $65 \mathrm{~kg}$. (RIVM, 2006a).

The Danish Environmental Protection Agency (D-EPA) has in their Guidance from the Environmental Protection Agency No. 5 (D-EPA, 2006) recommended a default value of $13 \mathrm{~kg}$ for children aged one to three years, and a default value of $70 \mathrm{~kg}$ for adults (D-EPA, 2006).

Table 2.1 presents a summary of body weights recommended by different European bodies.

Table 2.1: Default Values on Body Weights Recommended by Various European Bodies

\begin{tabular}{|c|c|}
\hline & Body Weight (kg) \\
\hline \multicolumn{2}{|l|}{ EFSA } \\
\hline $0-3$ months & 4.8 \\
\hline $3-6$ months & 6.7 \\
\hline $6-12$ months & 8.7 \\
\hline $1-3$ years & 11.6 \\
\hline $3-10$ years & 21.6 \\
\hline $10-14$ years & 42.0 \\
\hline $3-<6$ years & 17.5 \\
\hline $14-18$ years & 60.0 (both sexes combined), 65 (boys) / 56 (girls) \\
\hline Adults & 70 \\
\hline \multicolumn{2}{|c|}{ Commission of the European Communities (SCOOP data) } \\
\hline $0-<3$ years & 15 \\
\hline Adults & 60 \\
\hline \multicolumn{2}{|l|}{ ECHA } \\
\hline Adults & 70 (both sexes combined), 70 (men) / 60 (women) \\
\hline \multicolumn{2}{|r|}{ ק } \\
\hline 1.5 months & 4.30 \\
\hline 4.5 months & 6.21 \\
\hline 7.5 months & 7.62 \\
\hline 10.5 months & 8.69 \\
\hline 13.5 months & 9.47 \\
\hline 1.5 year & 9.85 \\
\hline 2.5 years & 12.5 \\
\hline 3.5 years & 14.1 \\
\hline 4.5 years & 16.3 \\
\hline 6.5 years & 20.6 \\
\hline 9.5 years & 28.4 \\
\hline 12.5 years & 39.3 \\
\hline 13.5 years & 43.9 \\
\hline 17.5 years & 58.2 \\
\hline Men & 74 \\
\hline Women & 61 \\
\hline Adults & 65 \\
\hline
\end{tabular}

Sources: EFSA (2011), Commission of the European Communities (2001), ECHA (2008a and 2008b) and RIVM (2006a) 
According to the Exposure Factors Sourcebook for European Populations, with Focus on UK Data (ECETOC, 2001), the recommended mean body weight for English adults aged 16 and older is $73.2 \mathrm{~kg}$. In Table 2.2, age and gender specific distributions of English adults are presented from data based on anthropometric measures. In Annex A, Table A.1, country specific estimates of adult (over 20 years of age) body weight are presented; the mean weights are based upon nationally representative data sets for about half of the countries listed, extrapolated to year 2000 based upon analysis of recent trends in body mass. For countries with no data, the WHO (which is the source of these data) used values from countries considered to be appropriate proxies (ECETOC, 2001).

Table 2.2: Recommended Values for Adult Body Weight - as Presented in the Exposure Factors Sourcebook for European Populations, with Focus on UK Data

\begin{tabular}{|c|c|c|c|c|c|c|}
\hline \multirow[t]{2}{*}{ Age (years) } & \multicolumn{3}{|c|}{ Men } & \multicolumn{3}{|c|}{ Women } \\
\hline & Mean (kg) & $5^{\text {th }}(\mathrm{kg})$ & $95^{\text {th }}(\mathrm{kg})$ & Mean (kg) & $5^{\text {th }}(\mathrm{kg})$ & $95^{\text {th }}(\mathrm{kg})$ \\
\hline $16-24$ & 72.8 & 54.5 & 97.1 & 62.7 & 47.8 & 85.9 \\
\hline $25-34$ & 80.7 & 60.9 & 105.6 & 67.0 & 50.0 & 91.3 \\
\hline $35-44$ & 82.4 & 63.1 & 107.6 & 67.8 & 50.4 & 93.7 \\
\hline $45-54$ & 82.7 & 62.3 & 106.5 & 69.3 & 50.9 & 94.3 \\
\hline $55-64$ & 82.8 & 63.4 & 107.3 & 70.7 & 52.0 & 95.0 \\
\hline $65-74$ & 78.9 & 60.0 & 100.5 & 68.2 & 48.2 & 92.0 \\
\hline $75+$ & 74.1 & 56.6 & 94.1 & 63.4 & 44.4 & 83.3 \\
\hline$\geq 16$ & 80.0 & 60.1 & 104.2 & 67.3 & 49.3 & 91.7 \\
\hline
\end{tabular}

Source: UK Office for National Statistics, 1998; modified from ECETOC, 2001

Table 2.3 presents mean body weight for children aged 2-15 years in England from the Exposure Factors Sourcebook for European Populations, with Focus on UK Data (ECETOC, 2001). The data are derived from the 1995-1997 Health Surveys for England. All data where measured during the survey and the recommended mean body weight is $33 \mathrm{~kg}$. For children younger than 2 years, ECETOC refers to the 1997 version of the Exposure Factors Handbook (US-EPA, 1997).

Table 2.3: Recommended Values for Child Body Weight - as Presented in the Exposure Factors Sourcebook for European Populations, with Focus on UK Data

\begin{tabular}{|c|c|c|c|c|c|c|}
\hline \multirow[t]{2}{*}{ Age (years) } & \multicolumn{3}{|c|}{ Males } & \multicolumn{3}{|c|}{ Females } \\
\hline & Mean (kg) & $5^{\text {th }}(\mathrm{kg})$ & $95^{\text {th }}(\mathrm{kg})$ & Mean (kg) & $5^{\text {th }}(\mathrm{kg})$ & $95^{\text {th }}(\mathrm{kg})$ \\
\hline 2 & 14.2 & 11.6 & 17.5 & 13.7 & 11.2 & 16.7 \\
\hline 3 & 16.4 & 13.4 & 20.2 & 16.0 & 12.8 & 20.2 \\
\hline 4 & 18.4 & 15.0 & 22.5 & 18.3 & 14.7 & 23.4 \\
\hline 5 & 20.4 & 16.3 & 25.5 & 20.4 & 16.2 & 25.5 \\
\hline 6 & 22.9 & 18.0 & 29.1 & 22.8 & 17.7 & 30.2 \\
\hline 7 & 25.8 & 20.5 & 33.7 & 25.9 & 19.8 & 37.9 \\
\hline 8 & 29.1 & 22.7 & 39.4 & 28.8 & 21.7 & 41.0 \\
\hline 9 & 32.0 & 24.0 & 43.9 & 32.7 & 24.2 & 45.7 \\
\hline 10 & 35.6 & 26.7 & 51.8 & 37.1 & 27.8 & 52.9 \\
\hline 11 & 40.2 & 29.3 & 55.2 & 42.4 & 28.9 & 62.3 \\
\hline 12 & 44.8 & 31.0 & 63.1 & 47.5 & 34.4 & 66.0 \\
\hline 13 & 50.8 & 35.0 & 71.6 & 51.8 & 38.9 & 70.6 \\
\hline 14 & 56.4 & 39.0 & 79.7 & 56.7 & 41.3 & 80.3 \\
\hline 15 & 62.9 & 47.1 & 85.6 & 58.4 & 43.6 & 79.2 \\
\hline $2-15$ & 32.9 & 14.3 & 64.6 & 32.8 & 13.8 & 62.6 \\
\hline
\end{tabular}

Source: UK Office for National Statistics, 1999, modified from ECETOC, 2001 


\subsection{US-EPA}

In the Exposure Factors Handbook (US-EPA, 2009), one "key study", a USEPA analysis of NHANES 1999-2006 data, is the basis for the recommended values for body weight, see Table 2.4. The data were collected through direct measurements during a physical examination. The mean body weight for all adults combined is $80.0 \mathrm{~kg}$. The US-EPA has noted that this mean recommended value is different from the $70 \mathrm{~kg}$ commonly assumed in the US-EPA risk assessments. The US-EPA therefore encouraged assessors to use the values which most accurately reflect the exposed population. When using values other than $70 \mathrm{~kg}$, however, the assessors should consider if the dose estimate will be used to estimate risk by combining it with a dose-response relationship which was derived assuming a body weight of $70 \mathrm{~kg}$. If such an inconsistency exists, the assessor should adjust the dose-response relationship. The Integrated Risk Information System (IRIS) does not use a $70 \mathrm{~kg}$ body weight assumption in the derivation of RfCs and RfDs, but does make this assumption in the derivation of cancer slope factors and unit risks. (US-EPA, 2009).

The values presented in Table 2.4 are well validated as the general assessment factors (GAFs, described in Section 1.2) were used to judge the quality of the underlying data used to derive the recommendations. The US-EPA has assigned the following confidence ratings for the five GAFs: 1. Soundness - high, 2. applicability and utility - high, 3. clarity and completeness - high, 4. variability and uncertainty - high, and 5. evaluation and review - medium; the overall rating was high. (US-EPA, 2009).

Values for body weight categorised by gender and for various adult age groups along with the $95^{\text {th }}$ percentiles are presented in the Annex A, Table A.2.

In addition to the key study, 11 "relevant studies" were identified and presented in the EFH; however, most of them are old and were therefore not considered as representative for the US population of today due to the steep increase in body weight. One study, from 2008, includes a large sample size and was therefore considered as representative of the US population for the age groups presented. Data from this study is presented in the Annex A, Table A.3. Data for birth weight are presented in the Annex A, Table A.4, based on another relevant study. 


\begin{tabular}{lccc}
\multicolumn{3}{l}{ Table 2.4: Recommended Values for Body Weight - as Presented in the Exposure Factors Handbook } \\
\hline Age group & Mean $\mathbf{( k g )}$ & $\mathbf{5}^{\text {th }}$ Percentiles & $\mathbf{9 5}^{\text {th }}$ Percentiles \\
\hline $0-<1$ month & 4.8 & 3.6 & 6.2 \\
$1-<$ months & 5.6 & 4.5 & 7.3 \\
$3-<6$ months & 7.4 & 5.7 & 9.1 \\
$6-<12$ months & 9.2 & 7.1 & 11.3 \\
$1-<2$ years & 11.4 & 8.9 & 14.0 \\
$2-<3$ years & 13.8 & 10.9 & 17.1 \\
$3-<6$ years & 18.6 & 13.5 & 26.2 \\
$6-<11$ years & 31.8 & 19.7 & 52.5 \\
$11-<16$ years & 56.8 & 34.0 & 88.8 \\
$16-<21$ years & 71.6 & 48.2 & 108.0 \\
Adults & 80.0 & - & -
\end{tabular}

Source: US-EPA analysis of NHANES, 1999-2006 data, modified from US-EPA, 2009

\section{$2.3 \mathrm{WHO}$}

WHO standard values for body weight are those recommended by the ICRP (ICRP, 1974, cited in WHO/IPCS, 1994 and 1999). These values are presented in Table 2.5. It should be noted that WHO uses $60 \mathrm{~kg}$ for calculation of acceptable daily intakes and water quality guidelines.

Table 2.5: WHO's Standard Values for Body Weight

\begin{tabular}{lr}
\hline Sex & Body Weight (kg) \\
\hline Men (Adults) & 70 \\
Women (Adults) & 58 \\
Men and Women Combined & $64^{\text {a }}$ \\
\hline
\end{tabular}

a) WHO uses $60 \mathrm{~kg}$ for calculation of acceptable daily intakes and water quality guidelines.

Source: Modified from WHO/IPCS, 1994 and 1999

\subsection{Conclusion and Recommendations}

The data presented in the US-EPA EFH (US-EPA, 2009) are the most comprehensive. The European data are more limited as are the WHO data.

In the REACH TGD Chapter R8 (ECHA, 2008a), the recommended values are stated as "taken from Gold et al., 1984 and ICRP, 1975"; however, it is not clear whether the recommendations are taken from one of these references or both. In the REACH TGD Chapter R15 (ECHA, 2008b) as well as in the report on the SCOOP data (Commission of the European Communities, 2001), no references for the recommended values are provided. The recommended values presented in the ConsExpo General Fact Sheet (RIVM, 2006a) are generated based upon data from the 1997 version of the EFH (US-EPA, 1997) and the recommendations from the ICRP (ICRP, 1992). The recommended values in ECETOC (ECETOC, 2001) are based on UK data published by the UK Office for National Statistics in 1998 (for adult body weight) and 1999 (for child body weight). The EFSA recommended values (EFSA, 2011) are taken from the EFSA 
Comprehensive European Food Consumption Database (Comprehensive Database) published on the EFSA website.

The US-EPA recommended values for body weights presented in Table 2.4 are well validated and valid as the general assessment factors (GAFs, described in Section 1.2) were used to judge the quality of the underlying data used to derive the recommendations and as the US-EPA overall confidence rating was high. (US-EPA, 2009).

WHO standard values for body weight are those recommended by the ICRP (ICRP, 1974).

It should be noted that the references "ICRP, 1974", "ICRP, 1975" and "ICRP, 1992" are to the same ICRP report (ICRP Report No. 23).

Americans tend, in average to weigh more than Europeans. Despite this, the US-EPA recommended values for children are considered as being representative for European children as well. Furthermore, the US-EPA recommended values are considered as being the most valid values for body weights as today as these values are based on an analyses of the most recent data (NHANES 1999-2006), whereas the European data are predominantly based on older references (ICRP, 1975; USEPA, 1997; UK Statistics from 1999) or no references have been provided for the recommended values (e.g., REACH TGD Chapter R15). For adults, the currently most used value for average body weight of $70 \mathrm{~kg}$ for men and women combined is probably more representative for Europeans than the average US adult body weight of $80 \mathrm{~kg}$. However, also in Europe, the adult body weight is increasing and therefore, probably approaching the American body weights of today.

In conclusion, the US-EPA recommended values presented in Table 2.4 are considered as being the most valid values for body weight as today and are therefore recommended as default exposure factors for assessments of European children in the context of REACH. For adults, however, the currently most used value for average body weight of $70 \mathrm{~kg}$ for men and women combined is still recommended as the default value for exposure assessments of the European population in the context of REACH. In addition to the average body weight for men and women combined, a default value of $70 \mathrm{~kg}$ for men and of $60 \mathrm{~kg}$ for women is recommended.

The recommended values can also be used in the context of other chemical regulations such as those for e.g., biocides, pesticides, cosmetics, toys etc. 


\section{Body Surface Areas}

Dermal exposure can occur during a variety of activities in different environmental media and microenvironments (US-EPA, 2009), including:

- Water (e.g., bathing, washing, swimming)

- Soil (e.g., outdoor recreation, gardening, construction)

- Sediment (e.g., wading, fishing)

- Liquids (e.g., use of commercial products)

- Vapours/fumes (e.g., use of commercial products) and

- Indoor dust (e.g., carpets, floors, counter tops)

Dermal exposure can also occur via contact with articles and materials.

Surface area of the skin can be determined using measurement or estimation techniques. Coating, triangulation, and surface integration are direct measurement techniques that have been used to measure total body surface area and the surface area of specific body parts. The coating method consists of coating either the whole body or specific body regions with a substance of known density and thickness. Triangulation consists of marking the area of the body into geometric figures, then calculating the figure areas from their linear dimensions. Surface integration is performed by using a planimeter and adding the areas. (US-EPA, 2009).

\subsection{Europe}

The recommendations provided in the REACH Guidance on information requirements and chemical safety assessment Chapter R15: Consumer exposure estimation (ECHA, 2008b) on body surface areas, taken from the 1997 version of the Exposure Factors Handbook (US-EPA, 1997), are presented in Table 3.1. 
Table 3.1: ECHA's Recommended Standard Values for Body Surface - as Presented in the REACH TGD Chapter R15

\begin{tabular}{lrr}
\hline Body Part & Men (mean, $\mathbf{c m}^{\mathbf{2}}$ ) & Women $\left(\mathbf{m e a n}, \mathbf{c m}^{\mathbf{2}}\right)$ \\
\hline Head (face) & 1,180 & 1,028 \\
Trunk & 5,690 & 4,957 \\
Upper Extremities & 3,190 & 2,779 \\
Arms & 2,280 & 1,984 \\
Upper Arms & 1,430 & 1,244 \\
Forearms & 1,140 & 992 \\
Hands (fronts and backs) & 840 & 731 \\
Lower Extremities & 6,360 & 5,533 \\
Legs & 5,060 & 4,402 \\
Thighs & 1,980 & 1,723 \\
Lower Legs & 2,070 & 1,801 \\
Feet & 1,120 & 1,001 \\
Total & 19,400 & 16,900 \\
\hline
\end{tabular}

Source: Exposure Factors Handbook, US-EPA, 1997, modified from ECHA, 2008b

In the General Fact Sheet (RIVM, 2006a), default values for body weight and surface area are generated based on data from the 1997 version of the Exposure Factors Handbook (US-EPA, 1997) and the ICRP Report of the Task Group on Reference Man (ICRP, 1992). Table B.1 in Annex B presents calculated default values generated upon the US-EPA bivariate equation (see below) and the US-EPA (1997) and ICRP data. The default values are $25^{\text {th }}$ percentiles.

For children, RIVM has also calculated default values for surface area, but by assuming that the relative surface area of the various parts of the body in children is dependent on age and not on weight or length, the default values are divided in age groups (RIVM, 2006a and 2002). Table B.2 in Annex B presents default values for children where the relative body surfaces "arms and legs" and "legs and feet" are not split up. Table B.3 presents default values for children where the data for "hands and arms" was split into the surface for the hands and the surface for the arms. For "legs and feet", the splitting up into "legs" and "feet" was carried out in a similar way. The data that these measurements are based upon include different age groups than in Table B.2, therefore, the age groups are different in Table B.3. This is also the reason for presenting two tables instead of just one.

According to the Exposure Factors Sourcebook for European Populations, with Focus on UK Data (ECETOC, 2001), skin surface area can be calculated using the US-EPA bivariate equation for populations in which both body weight and height are known:

\section{US-EPA bivariate equation:}

$\mathrm{SA}=\mathrm{a} \mathrm{H} \mathrm{H}^{\mathrm{b}} \mathrm{W}^{\mathrm{c}}$, where

$\mathrm{SA}=$ Surface Area $\left(\mathrm{m}^{2}\right)$

$\mathrm{H}=$ Height $(\mathrm{cm})$

$\mathrm{W}=$ Weight $(\mathrm{kg})$

$\mathrm{a}, \mathrm{b}$ and $\mathrm{c}$ are constants $(\mathrm{a}=0.0239, \mathrm{~b}=0.417$ and $\mathrm{c}=0.517)$

Source: US-EPA, 1989 - based on data from Murray and Burmaster, 1992. 
Alternatively, when only body weight data are available, skin surface area can be calculated using either the equation of Costeff or Burmaster:

\section{Costeff Equation:}

$S A=(4 W+7) /(W+90)$, where

$\mathrm{SA}=$ Surface Area $\left(\mathrm{m}^{2}\right)$

$\mathrm{W}=$ Weight $(\mathrm{kg})$

Source: Costeff, 1966 in US-EPA (1997), based upon 220 observations of children

\section{Burmasters Equation:}

$\mathrm{SA}=\mathrm{a} * \mathrm{BWc}$ or $\ln \mathrm{SA}=\ln \mathrm{a}+\mathrm{c} \ln \mathrm{BW}$, where

SA $=$ Skin Surface Area

BW $=$ Body Weight, and:

$\ln \mathrm{a}=-2.2781, \mathrm{c}=0.6821$ for all 401 people

$\ln \mathrm{a}=-2.2752, \mathrm{c}=0.6868$ for males

$\ln \mathrm{a}=-2.2678, \mathrm{c}=0.6754$ for females

Source: Burmaster, 1998, based upon 401 observations of adults and children

According to ECETOC (ECETOC, 2001), the equation of Burmaster may give a better estimate of central values than that of Costeff, but overestimates skin surface area at upper values. Based upon mean English adult body weight and the equation of Burmaster, total skin surface area is estimated as $2.07 \mathrm{~m}^{2}$ for males and $1.76 \mathrm{~m}^{2}$ for females, with an average of $1.92 \mathrm{~m}^{2}$ (ECETOC, 2001). Distributions for several gender and age groups are presented in Annex B, Table B.4. Furthermore, ECETOC refers to the 1997 version of the Exposure Factors Handbook (US-EPA, 1997) for the use of body surface area standard values, see Table 3.1.

The clothes people wear function as protection against various types of exposure. The fraction of exposed skin surface depends upon climate and other aspects specific to a given exposure scenario, such as type of activity. Suggested defaults values of the percentage of the body that is exposed during some exposure scenarios from Exposure Factors Sourcebook for European Populations, with Focus on UK Data (ECETOC, 2001), are listed in Annex B, Table B.5. However, all kind of clothes are not 100 percent protective. Therefore, in the Technical Notes for Guidance on Human Exposure to Biocidal Products, TNsG (2007), default values of the protection factor of different sort of outfits are given and are listed in Annex B, Table B.6.

\subsection{US-EPA}

In the Exposure Factors Handbook (EFH) (US-EPA, 2009), two "key studies" and 3 "relevant studies" were identified. The recommendations for total body surface area are based on the most recent key study, which is a USEPA analysis of weight and height 1999-2006 data from the National Health 
and Nutrition Examination Survey (NHANES) for children under age 21 years and are presented for the standard age groupings recommended by US-EPA (US-EPA, 2005) for male and female children combined. For adults 21 years and over, the recommendations for total body surface area are based on a US-EPA analysis of NHANES 2005-2006 data. Recommended values for total body surface area are presented In Table 3.2. The recommendations for the percentage of total body surface area represented by individual body parts are presented in Table 3.3.

The values presented in Table 3.2 and 3.3 are well validated as the general assessment factors (GAFs, described in Section 1.2) were used to judge the quality of the underlying data used to derive the recommendations. The US-EPA has assigned the following confidence ratings for the five GAFs: 1 . Soundness - medium, 2. applicability and utility - medium, 3. clarity and completeness - medium, 4. variability and uncertainty medium, and 5. evaluation and review - medium; the overall rating was medium for "total surface area" and low for "surface area of individual body parts." (US-EPA, 2009).

In Annex B, Table B.7 presents the mean percent of surface area for children categorized per year based upon data from the abovementioned studies and Table B.8 presents surface area of adults (21+ years) in square meters in more detailed body parts than those presented in Table 3.3.

\begin{tabular}{|c|c|c|}
\hline Age (group) & Mean $\left(\mathrm{m}^{2}\right)$ & $95^{\text {th }}$ Percentile $\left(\mathrm{m}^{2}\right)$ \\
\hline \multicolumn{3}{|l|}{ Children } \\
\hline 0-1 month & 0.29 & 0.34 \\
\hline $1-<3$ months & 0.33 & 0.38 \\
\hline $3-<6$ months & 0.38 & 0.44 \\
\hline $6-<12$ months & 0.45 & 0.51 \\
\hline $1-<2$ years & 0.53 & 0.61 \\
\hline $2-<3$ years & 0.61 & 0.70 \\
\hline $3-<6$ years & 0.76 & 0.95 \\
\hline $6-<11$ years & 1.08 & 1.48 \\
\hline $11-<16$ years & 1.59 & 2.06 \\
\hline $16-<21$ years & 1.84 & 2.33 \\
\hline \multicolumn{3}{|l|}{ Adult Men } \\
\hline $21-<30$ years & 2.05 & 2.52 \\
\hline $30-<40$ years & 2.10 & 2.50 \\
\hline $40-<50$ years & 2.15 & 2.56 \\
\hline $50-<60$ years & 2.11 & 2.55 \\
\hline $60-<70$ years & 2.08 & 2.46 \\
\hline $70-<80$ years & 2.05 & 2.45 \\
\hline$\geq 80$ years & 1.92 & 2.22 \\
\hline \multicolumn{3}{|l|}{ Adult Women } \\
\hline $21-<30$ years & 1.81 & 2.25 \\
\hline $30-<40$ years & 1.85 & 2.31 \\
\hline $40-<50$ years & 1.88 & 2.36 \\
\hline $50-<60$ years & 1.89 & 2.38 \\
\hline $60-<70$ years & 1.88 & 2.34 \\
\hline $70-<80$ years & 1.77 & 2.13 \\
\hline$\geq 80$ years & 1.69 & 1.98 \\
\hline
\end{tabular}

Source: US-EPA Analysis of NHANES 1999-2006 data, modified from US-EPA, 2009. 
Table 3.3: The values in the table have been changed according to the final EPA report. Recommended Values of Surface Area of Body Parts for Children (Genders Combined) and Adults by Gender - as Presented in the Exposure Factors Handbook

\begin{tabular}{|c|c|c|c|c|c|c|}
\hline \multirow[t]{2}{*}{ Age } & Head & Trunk & Arms & Hands & Legs & Feet \\
\hline & \multicolumn{6}{|c|}{ Mean Percent of Total Surface Area (\%) } \\
\hline \multicolumn{7}{|l|}{ Children } \\
\hline $0-1 \mathrm{~m}$ & 18.2 & 35.7 & 13.7 & 5.3 & 20.6 & 6.5 \\
\hline $1-<3 \mathrm{~m}$ & 18.2 & 35.7 & 13.7 & 5.3 & 20.6 & 6.5 \\
\hline $3-<6 \mathrm{~m}$ & 18.2 & 35.7 & 13.7 & 5.3 & 20.6 & 6.5 \\
\hline $6-<12 m$ & 18.2 & 35.7 & 13.7 & 5.3 & 20.6 & 6.5 \\
\hline $1-<2 y$ & 16.5 & 35.5 & 13.0 & 5.7 & 23.1 & 6.3 \\
\hline $2-3 y$ & 8.4 & 41.0 & 14.4 & 4.7 & 25.3 & 6.3 \\
\hline $3-<6 y$ & 8.0 & 41.2 & 14.0 & 4.9 & 25.7 & 6.4 \\
\hline $6-<11$ y & 6.1 & 39.6 & 14.0 & 4.7 & 28.8 & 6.8 \\
\hline $11-<16 y$ & 4.6 & 39.6 & 14.3 & 4.5 & 30.4 & 6.6 \\
\hline $16-<21 y$ & 4.1 & 41.2 & 14.6 & 4.5 & 29.5 & 6.1 \\
\hline \multicolumn{7}{|c|}{ Adult Men } \\
\hline$\geq 21 \mathrm{y}$ & 6.6 & 40.1 & 15.2 & 5.2 & 33.1 & 6.7 \\
\hline \multicolumn{7}{|c|}{ Adult Women } \\
\hline$\geq 21 \mathrm{y}$ & 6.2 & 35.4 & 12.8 & 4.8 & 32.3 & 6.6 \\
\hline
\end{tabular}

\begin{tabular}{|c|c|c|c|c|c|c|c|c|c|c|c|c|}
\hline \multicolumn{13}{|c|}{ Surface Area $\left(\mathrm{m}^{2}\right)$} \\
\hline & Mean $^{a}$ & $95^{\text {th b }}$ & Mean $^{a}$ & $95^{\text {th b }}$ & Mean $^{\mathrm{a}}$ & $95^{\text {th b }}$ & Mean $^{\mathrm{a}}$ & $95^{\text {th b }}$ & Mean $^{\mathrm{a}}$ & $95^{\text {th b }}$ & Mean $^{a}$ & $95^{\text {th b }}$ \\
\hline \multicolumn{13}{|l|}{ Children } \\
\hline 0-1 m & 0.053 & 0.062 & 0.104 & 0.121 & 0.040 & 0.047 & 0.015 & 0.018 & 0.060 & 0.070 & 0.019 & 0.022 \\
\hline $1-<3 m$ & 0.060 & 0.069 & 0.118 & 0.136 & 0.045 & 0.052 & 0.017 & 0.020 & 0.068 & 0.078 & 0.021 & 0.025 \\
\hline $3-<6 \mathrm{~m}$ & 0.069 & 0.080 & 0.136 & 0.157 & 0.052 & 0.060 & 0.020 & 0.023 & 0.078 & 0.091 & 0.025 & 0.029 \\
\hline $6-<12 \mathrm{~m}$ & 0.082 & 0.093 & 0.161 & 0.182 & 0.062 & 0.070 & 0.024 & 0.027 & 0.093 & 0.105 & 0.029 & 0.033 \\
\hline $1-<2 y$ & 0.087 & 0.101 & 0.188 & 0.217 & 0.069 & 0.079 & 0.030 & 0.035 & 0.122 & 0.141 & 0.033 & 0.038 \\
\hline $2-<3 y$ & 0.051 & 0.059 & 0250 & 0.287 & 0.088 & 0101 & 0.028 & 0.033 & 0.154 & 0.177 & 0.038 & 0.044 \\
\hline $3-<6$ y & 0.061 & 0.076 & 0.313 & 0.391 & 0.106 & 0.133 & 0.037 & 0.046 & 0.195 & 0.244 & 0.049 & 0.061 \\
\hline $6-<11$ y & 0.066 & 0.090 & 0.428 & 0.586 & 0.151 & 0.207 & 0.051 & 0.070 & 0.311 & 0.426 & 0.073 & 0.100 \\
\hline $11-<16$ y & 0.073 & 0.095 & 0630 & 0.816 & 0.227 & 0.295 & 0.072 & 0.093 & 0.483 & 0.626 & 0.105 & 0.136 \\
\hline $16-<21 y$ & 0.075 & 0.096 & 0.759 & 0.960 & 0.269 & 0340 & 0.083 & 0.105 & 0.543 & 0.687 & 0.112 & 0.142 \\
\hline \multicolumn{13}{|c|}{ Adult Men } \\
\hline$\geq 21 \mathrm{y}$ & 0.136 & 0.154 & 0.827 & 1.100 & 0.314 & 0.399 & 0.107 & 0.131 & 0.682 & 0.847 & 0.137 & 0.161 \\
\hline \multicolumn{13}{|c|}{ Adult Women } \\
\hline$\geq 21 \mathrm{y}$ & 0.114 & 0.121 & 0.654 & 0.850 & 0.237 & 0.266 & 0.089 & 0.106 & 0.598 & 0.764 & 0.122 & 0.146 \\
\hline
\end{tabular}

a) Calculated as mean percentage of body part times mean total body surface area.

b) Calculated as mean percentage of body part times $95^{\text {th }}$ percentile total body surface area.

Note: Surface area values reported in $\mathrm{m}^{2}$ can be converted to $\mathrm{cm}^{2}$ by multiplying by $10,000 \mathrm{~cm}^{2} / \mathrm{m}^{2}$.

Source: US-EPA Analysis of NHANES 1999-2006 data and US-EPA, 1985, modified from US-EPA, 2009.

Please note that there are no changes for adults. 


\section{$3.3 \mathrm{WHO}$}

No recommendations on body surface area are provided by WHO.

\subsection{Conclusion and Recommendations}

The data presented in the US-EPA EFH (US-EPA, 2009) as well as the European data are comprehensive. No recommendations have been provided by the WHO.

In the REACH TGD Chapter R15 (ECHA, 2008b), the recommended values presented in Table 3.1 are taken from the 1997 version of the EFH (US-EPA, 1997). The recommended values presented in the ConsExpo General Fact Sheet (RIVM, 2006a) are generated based upon data from the 1997 version of the EFH (US-EPA, 1997) and the recommendations from the ICRP (ICRP, 1992). It should be noted that the reference "ICRP, 1992" is to the "ICRP, 1974" / "ICRP, 1975" report (ICRP Report No. 23). The recommended values from ECETOC (ECETOC, 2001) are calculated based upon UK body weight data and different equations. Furthermore, ECETOC refers to the 1997 version of the EFH (US-EPA, 1997) for the use of body surface area standard values.

The US-EPA recommended values for body surface areas presented in Table 3.2 and 3.3 are well validated as the general assessment factors (GAFs, described in Section 1.2) were used to judge the quality of the underlying data used to derive the recommended values. The US-EPA overall rating was medium for "total surface area" and low for "surface area of individual body parts". (US-EPA, 2009).

Americans tend, in average, to weigh more and thus to have a greater body surface area than Europeans. Despite this, the US-EPA recommended values are considered as being representative for Europeans as well. Furthermore, the US-EPA recommended values are considered as being the most valid values for body surface areas as today as these values are based on analyses of the most recent data (NHANES 19992006), whereas the European data are predominantly based on older data, primarily the 1997 version of the EFH (US-EPA, 1997). However, also in Europe, the adult body weight is increasing and thus, also the body surface area and therefore, probably approaching the American body weights and body surface area of today.

In conclusion, the US-EPA recommended values presented in Table 3.2 and 3.3 are considered as being the most valid values for body surface areas as today and are therefore recommended as default exposure factors for assessments of the European population in the context of REACH.

The recommended values can also be used in the context of other chemical regulations such as those for e.g., biocides, pesticides, cosmetics, toys etc. 


\section{Inhalation rates}

Outdoor (ambient) and indoor air are potential sources of exposure to toxic substances. Adults and children can be exposed to contaminated air during a variety of activities in different environments. They may be exposed to contaminants in ambient air, and may also inhale chemicals from the indoor use of various consumer products. (US-EPA, 2009).

Due to their size, physiology, and activity level, the inhalation rates of children differ from those of adults. Infants and children have a higher resting metabolic rate and oxygen consumption rate per unit of body weight than adults, because of their rapid growth and relatively larger lung surface area per unit of body weight that requires cooling. Thus, while greater amounts of air and pollutants are inhaled by adults than children over similar time periods on an absolute basis, the volume of air passing through the lungs of a resting infant is up to twice that of a resting adult on a body weight basis. (US-EPA, 2009).

\subsection{Europe}

In the REACH Guidance on information requirements and chemical safety assessment Chapter R8 (ECHA, 2008a), an inhalation volume of 20 $\mathrm{m}^{3}$ /day is recommended for exposure assessments of consumers and man exposed indirectly via the environment, and of $10 \mathrm{~m}^{3} / 8$ hours light work for exposure assessments of workers (Table R. 8-18).

The recommendations provided in the REACH Guidance on information requirements and chemical safety assessment Chapter R8 (ECHA, 2008a) Table R. 8-2 and in the REACH Guidance on information requirements and chemical safety assessment Chapter R15: Consumer exposure estimation (ECHA, 2008b) Tables R.15-14 to R.15-16 on respiratory volumes are presented in Table 4.1. 
Table 4.1: ECHA's Recommended Standard Values for Respiratory Volume - as Presented in the REACH TGD Chapter R8 and R15

\begin{tabular}{|c|c|}
\hline Body Weight ${ }^{a, b}$ & $70 \mathrm{~kg}$ \\
\hline Respiratory Volume ${ }^{b}$ & $0.2 \mathrm{~L} / \mathrm{min} / \mathrm{kg}$ \\
\hline \multicolumn{2}{|l|}{ For relevant duration ${ }^{b}$} \\
\hline 6 hour exposure & $5 \mathrm{~m}^{3} /$ person \\
\hline 8 hour exposure & $6.7 \mathrm{~m}^{3} /$ person \\
\hline 24 hour exposure & $20 \mathrm{~m}^{3} /$ person \\
\hline \multicolumn{2}{|c|}{ Respiratory Volume Light Activity for Worker ${ }^{a, b}$} \\
\hline 8 hour exposure & $10 \mathrm{~m}^{3} /$ person \\
\hline \multicolumn{2}{|c|}{ Respiratory Volume Related to Activity Level ${ }^{c}$} \\
\hline \multicolumn{2}{|c|}{ (Men:20-30 years; Women: $20-33$ years) } \\
\hline \multicolumn{2}{|l|}{ Resting } \\
\hline Men & $6.5-10.8 \mathrm{~m}^{3} /$ day \\
\hline Women & $6.5-8.6 \mathrm{~m}^{3} /$ day \\
\hline Pregnant Women & $14 \mathrm{~m}^{3} /$ day \\
\hline \multicolumn{2}{|l|}{ Light Activity } \\
\hline Men & $29-42 \mathrm{~m}^{3} /$ day \\
\hline Women & $23-27 \mathrm{~m}^{3} / \mathrm{day}$ \\
\hline \multicolumn{2}{|l|}{ Medium Activity } \\
\hline Men & $62 \mathrm{~m}^{3} /$ day \\
\hline Women & $36 \mathrm{~m}^{3} /$ day \\
\hline \multicolumn{2}{|l|}{ Heavy Activity } \\
\hline Men & $160 \mathrm{~m}^{3} /$ day \\
\hline Women & $130 \mathrm{~m}^{3} /$ day \\
\hline \multicolumn{2}{|c|}{ Respiratory Volume for Short-Term Exposure ${ }^{c}$} \\
\hline \multicolumn{2}{|l|}{ Resting, Children } \\
\hline$<1$ year & $1.4 \mathrm{~m}^{3} / \mathrm{day}$ \\
\hline $1-3$ years & $2.9 \mathrm{~m}^{3} / \mathrm{day}$ \\
\hline $4-6$ years & $5.8 \mathrm{~m}^{3} / \mathrm{day}$ \\
\hline $7-9$ years & $8.6 \mathrm{~m}^{3} /$ day \\
\hline $10-14$ years & $12 \mathrm{~m}^{3} /$ day \\
\hline \multicolumn{2}{|l|}{ Resting, Adolescents } \\
\hline $15-19$ years & $13 \mathrm{~m}^{3} /$ day \\
\hline \multicolumn{2}{|l|}{ Resting, Adults } \\
\hline 20-75 years & $13 \mathrm{~m}^{3} /$ day \\
\hline \multicolumn{2}{|l|}{ Light Activity, Children } \\
\hline$<1$ year & $2.9 \mathrm{~m}^{3} / \mathrm{day}$ \\
\hline $1-3$ years & $5.8 \mathrm{~m}^{3} / \mathrm{day}$ \\
\hline $4-6$ years & $12 \mathrm{~m}^{3} /$ day \\
\hline $7-9$ years & $12 \mathrm{~m}^{3} /$ day \\
\hline $10-14$ years & $23 \mathrm{~m}^{3} /$ day \\
\hline \multicolumn{2}{|c|}{ Light Activity, Adolescents } \\
\hline $15-19$ years & $26 \mathrm{~m}^{3} /$ day \\
\hline \multicolumn{2}{|l|}{ Light Activity, Adults } \\
\hline 20-75 years & $26 \mathrm{~m}^{3} /$ day \\
\hline \multicolumn{2}{|c|}{ Medium Activity, Children } \\
\hline$<1$ year & $5.8 \mathrm{~m}^{3} /$ day \\
\hline $1-3$ years & $12 \mathrm{~m}^{3} /$ day \\
\hline $4-6$ years & $23 \mathrm{~m}^{3} /$ day \\
\hline $7-9$ years & $35 \mathrm{~m}^{3} /$ day \\
\hline $10-14$ years & $46 \mathrm{~m}^{3} / \mathrm{day}$ \\
\hline Medium Activity, Adole & \\
\hline $15-19$ years & $51 \mathrm{~m}^{3} /$ day \\
\hline Medium Activity, Adult & \\
\hline 20-75 years & $51 \mathrm{~m}^{3} /$ day \\
\hline Heavy Activity, Childrer & \\
\hline$<1$ year & $10 \mathrm{~m}^{3} /$ day \\
\hline $1-3$ years & $20 \mathrm{~m}^{3} /$ day \\
\hline $4-6$ years & $40 \mathrm{~m}^{3} /$ day \\
\hline $7-9$ years & $61 \mathrm{~m}^{3} /$ day \\
\hline $10-14$ years & $81 \mathrm{~m}^{3} /$ day \\
\hline Heavy Activity, Adolesc & \\
\hline $15-19$ years & $91 \mathrm{~m}^{3} /$ day \\
\hline Heavy Activity, Adults & \\
\hline $20-75$ years & $91 \mathrm{~m}^{3} /$ day \\
\hline
\end{tabular}




\begin{tabular}{lr}
\hline Body Weight $^{\text {a,b }}$ & $\mathbf{7 0 ~} \mathbf{k g}$ \\
\hline $\begin{array}{lr}\text { Respiratory Volume for a Whole Day Exposure }{ }^{\mathrm{c}} \\
\text { Children }\end{array}$ \\
$<1$ year & $3 \mathrm{~m}^{3} / \mathrm{day}$ \\
$1-3$ years & $7 \mathrm{~m}^{3} / \mathrm{day}$ \\
$4-6$ years & $11 \mathrm{~m}^{3} / \mathrm{day}$ \\
$7-9$ years & $14 \mathrm{~m}^{3} / \mathrm{day}$ \\
$10-14$ years & $18 \mathrm{~m}^{3} / \mathrm{day}$ \\
Adolescents & \\
$15-19$ years & $20 \mathrm{~m}^{3} /$ day \\
Adults & \\
$20-75$ years & $18 \mathrm{~m}^{3} / \mathrm{day}$ \\
\hline
\end{tabular}

Sources: a: ECHA, 2008a (Taken from Gold et al., 1984 and ICRP, 1975 - cited in ECHA, 2008a); b: ECHA, 2008a (no reference(s) provided for default physiological parameters in Table R. 8-2 - cited in ECHA, 2008a); and c: ECHA, 2008b (Taken from AUH, 1995 - cited in ECHA, 2008b)

In the Preparation of a Guidance Document on Pesticide Exposure; Assessment for Workers, Operators, Bystanders and Residents (EFSA, 2010), the recommendations regarding inhalation rates are based upon the data from the 2009 version of the US-EPA Exposure Factors Handbook (US-EPA, 2009) and are presented in Table 4.2.

Table 4.2: Inhalation Rates recommended by EFSA

\begin{tabular}{lc}
\hline Age group & Inhalation rate \\
\hline Daily Inhalation Rate; long term exposure & \\
$<1$ year & $1.14 \mathrm{~m}^{3} /$ day $/ \mathrm{kg}$ \\
$1-3$ years & $1.07 \mathrm{~m}^{3} /$ day $/ \mathrm{kg}$ \\
$3-<6$ years & $0.70 \mathrm{~m}^{3} /$ day $/ \mathrm{kg}$ \\
$6-<11$ years & $0.44 \mathrm{~m}^{3} /$ day $/ \mathrm{kg}$ \\
$11-<16$ years & $0.27 \mathrm{~m}^{3} /$ day $/ \mathrm{kg}$ \\
Adults & $0.23 \mathrm{~m}^{3} /$ day $/ \mathrm{kg}$ \\
Short term exposure (<30 min); high intensity hourly & exposure \\
& \\
$<1$ year & $0.196 \mathrm{~m}^{3} / \mathrm{hour} / \mathrm{kg}$ \\
$1-<3$ years & $0.190 \mathrm{~m}^{3} / \mathrm{hour} / \mathrm{kg}$ \\
$3-<6$ years & $0.120 \mathrm{~m}^{3} / \mathrm{hour} / \mathrm{kg}$ \\
$6-<11$ years & $0.082 \mathrm{~m}^{3} / \mathrm{hour} / \mathrm{kg}$ \\
$11-<16$ years & $0.055 \mathrm{~m}^{3} / \mathrm{hour} / \mathrm{kg}$ \\
Adults & $0.040 \mathrm{~m}^{3} / \mathrm{hour} / \mathrm{kg}$ \\
\hline
\end{tabular}

Source: EFSA, 2010

The Danish Environmental Protection Agency (D-EPA) has in their Guidance from the Environmental Protection Agency No. 5 (D-EPA, 2006) based their recommended inhalation rate upon data in the 1997 version of the US-EPA Exposure Factors Handbook (US-EPA, 1997). A default value $0.5 \mathrm{~m}^{3}$ air $/ \mathrm{kg}$ bw for children aged one to five is recommended (DEPA, 2006).

In the Exposure Factors Sourcebook for European Populations, with Focus on UK Data (ECETOC, 2001), the recommended long-term and short-term inhalation rates are taken from the 1997 version of the Exposure Factors Handbook (US-EPA, 1997). ECETOC has noted that these values are based upon US data, but are probably representative of Europeans as well, see Table 4.3 and 4.4. 
Table 4.3: Values for Long-term Inhalation Rates recommended by ECETOC - as Presented in the Exposure Factors Sourcebook for European Populations, with Focus on UK Data

\begin{tabular}{llr}
\hline Population & Gender & Mean $\mathbf{m}^{\mathbf{3}} /$ day \\
\hline Infants: & & 4.5 \\
$<1$ year & - & \\
Children: & - & 6.8 \\
$1-2$ years & - & 8.3 \\
$3-5$ years & - & 10.0 \\
$6-8$ years & Female & 13.0 \\
$9-11$ years & Male & 14.0 \\
$9-11$ years & Female & 12.0 \\
$12-14$ years & Male & 15.0 \\
$12-14$ years & Female & 12.0 \\
$15-18$ years & Male & 17.0 \\
$15-18$ years & & \\
Adults: & Female & 11.3 \\
$19-65$ years & Male & 15.2 \\
$19-65$ years & &
\end{tabular}

Source: US-EPA, 1997, modified from ECETOC, 2001

Table 4.4: Values for Short-term Inhalation Rates recommended by ECETOC - as Presented in the Exposure Factors Sourcebook for European Populations, with Focus on UK Data

\begin{tabular}{llr}
\hline Population & Activity Level & Mean $\mathbf{m}^{\mathbf{3}} /$ hour \\
\hline Adults: & & \\
& Rest & 0.4 \\
& Sedentary & 0.5 \\
& Light & 1.0 \\
& Moderate & 1.6 \\
& Heavy & 3.2 \\
Children: & Rest & \\
& Sedentary & 0.3 \\
& Light & 0.4 \\
& Moderate & 1.0 \\
& Heavy & 1.2 \\
& & 1.9 \\
Outdoor Workers: & Slow & 1.1 \\
& Moderate & 1.5 \\
& Heavy & 2.5 \\
& Hourly Average & 3.3 )
\end{tabular}

Source: US-EPA, 1997, modified from ECETOC, 2001

\subsection{US-EPA}

In the Exposure Factors Handbook (EFH) (US-EPA, 2009), four "key studies" and 13 "relevant studies" were identified. The recommended inhalation rates for adults and children are based on three recent key studies as well as an additional recent study of children. The US-EPA has noted that these key studies represent an improvement upon those previously used for recommended inhalation rates in previous versions of the EFH, because they have used a large data set that is representative of the US as a whole and consider the correlation between body weight and inhalation rate (US-EPA, 2009). It was also noted that the selection of inhalation rates to be used for exposure assessments depends on the age of the 
exposed population and the specific activity levels of this population during various exposure scenarios (US-EPA, 2009).

The recommended long-term inhalation rates $\left(\mathrm{m}^{3} /\right.$ day) for adults and children (including infants) for use in various exposure scenarios are presented in Table 4.5. The values are at least averages of two of the key studies. The US-EPA (US-EPA, 2009) has defined long-term exposure as "repeated exposure for more than 30 days, up to approximately $10 \%$ of the life span in humans (more than 30 days)" The US-EPA has noted that all of the $95^{\text {th }}$ percentile values represent unusually high inhalation rates for long-term exposures, even for the upper end of the distribution, but that they were included in the EFH to provide exposure assessors a sense of the possible range of inhalation rates for adults and children. Therefore, the US-EPA advices that these values should be used with caution when estimating long-term exposures.

Table 4.5: Recommended Long-Term Exposure Values for Inhalation - as Presented in the Exposure Factors Handbook

\begin{tabular}{|c|c|c|}
\hline & Mean $\mathrm{m}^{3} /$ day & $95^{\text {th }}$ Percentile $\mathrm{m}^{3} /$ day \\
\hline \multicolumn{3}{|l|}{ Infants: } \\
\hline Birth to 1 month & 3.6 & 7.1 \\
\hline $1-<3$ months & 3.5 & 5.8 \\
\hline $3-<6$ months & 4.1 & 6.1 \\
\hline $6-<12$ months & 5.4 & 8.0 \\
\hline Birth to $<1$ year & 5.4 & 9.2 \\
\hline \multicolumn{3}{|l|}{ Children: } \\
\hline $1-<2$ years & 8.0 & 12.8 \\
\hline $2-<3$ years & 8.9 & 13.7 \\
\hline $3-<6$ years & 10.1 & 13.8 \\
\hline $6-<11$ years & 12.0 & 16.6 \\
\hline $11-<16$ years & 15.2 & 21.9 \\
\hline $16-<21$ years & 16.3 & 24.6 \\
\hline \multicolumn{3}{|c|}{ Adults (over 21 years): } \\
\hline $21-<31$ years & 15.7 & 21.3 \\
\hline $31-<41$ years & 16.0 & 21.4 \\
\hline $41-<51$ years & 16.0 & 21.2 \\
\hline $51-<61$ years & 15.7 & 21.3 \\
\hline $61-<71$ years & 14.2 & 18.1 \\
\hline $71-<81$ years & 12.9 & 16.6 \\
\hline$\geq 81$ years & 12.2 & 15.7 \\
\hline
\end{tabular}

Source: Modified from US-EPA, 2009

In order to present more detailed information of long-term inhalation rates stratified by sex and weight, additional tables are included in Annex C: Table C.1 presents daily inhalation rates from free-living (opposite to staged activity measures) normal-weight men and women. Table C. 2 and C. 3 present daily physiological inhalation rates (a specific method for estimation of inhalation rates, calculated as described in footnote $\mathrm{b}$ to Table C.2) for free-living normal-weight men and women aged 2.6 months to 96 years, and for free-living normal-weight and overweight/obese men and women aged 4 to 96 years, respectively. Table C.4 and C.5 are equal to Table C.2 and C.3, but adjusted by body weight. Table C.6 presents daily physiological inhalation rates for newborns 
aged one month or less. Table C.1 to C.6 are all based upon one single key study (Brochu et al., 2006, in the 2009 version of the EFH). Table C.7 and C.8 present inhalation rates for men and women unadjusted and adjusted for body weight, respectively, based upon another single key study (US-EPA, 2009, in the 2009 version of the EFH).

The recommended short-term inhalation rates $\left(\mathrm{m}^{3} /\right.$ minute) are presented in Table 4.6 for men and women combined, for adults and children for which activity patterns are known. These values represent averages of the activity level data from the one key study from which short-term inhalation rate data were available. The US-EPA (US-EPA, 2009) has defined short-term exposure as "repeated exposure for more than 24 hours, up to 30 days."

Each activity pattern was assigned a metabolic equivalents (METS) value based on statistical sampling of the distribution assigned by CHAD (the US-EPA's Consolidated Human Activity Database) to each activity code. The inhalation rate for each activity within the 24-hour simulated activity pattern for each individual was estimated as a function of $\mathrm{VO}_{2}$ (oxygen consumption rate), body weight, age, and gender. Following this, the average inhalation rate was calculated for each individual for the entire 24-hour period, as well as for four separate classes of activities based on METS value: Sedentary/passive, METS less than or equal to 1.5; light intensity, METS greater than 1.5 and less than or equal to 3.0; moderate intensity, METS greater than 3.0 and less than or equal to 6.0; and high intensity, METS greater than 6.0 (US-EPA, 2009). In the Annex, short-term inhalation rates for gender and age categories on a volumetric $\left(\mathrm{m}^{3} /\right.$ day) and body-weight adjusted $\left(\mathrm{m}^{3} /\right.$ day- $\left.\mathrm{kg}\right)$ basis are presented Table C.9 and C.10, respectively. 
Table 4.6: Recommended Short-Term Exposure Values for Inhalation Rates ${ }^{a}$ - as Presented in the Exposure Factors Handbook

\begin{tabular}{|c|c|c|c|c|c|c|c|c|c|c|}
\hline \multirow[t]{2}{*}{ Age (years) } & \multicolumn{2}{|c|}{ Sleep or Nap } & \multicolumn{2}{|c|}{ Sedentary/Passive } & \multicolumn{2}{|c|}{ Light Intensity } & \multicolumn{2}{|c|}{ Moderate Intensity } & \multicolumn{2}{|c|}{ High Intensity } \\
\hline & Mean $\mathrm{m}^{3} / \mathrm{min}$ & $95^{\text {th }} \mathrm{m}^{3} / \mathrm{min}$ & Mean $\mathrm{m}^{3} / \mathrm{min}$ & $95^{\text {th }} \mathrm{m}^{3} / \mathrm{min}$ & Mean $\mathrm{m}^{3} / \mathrm{min}$ & $95^{\text {th }} \mathrm{m}^{3} / \mathrm{min}$ & Mean $\mathrm{m}^{3} / \mathrm{min}$ & $95^{\text {th }} \mathrm{m}^{3} / \mathrm{min}$ & Mean $\mathrm{m}^{3} / \mathrm{min}$ & $95^{\text {th }} \mathrm{m}^{3} / \mathrm{min}$ \\
\hline $0-<1$ & $3.0 \mathrm{E}-03$ & $4.6 \mathrm{E}-03$ & $3.1 \mathrm{E}-03$ & $4.7 \mathrm{E}-03$ & $7.6 \mathrm{E}-03$ & $1.1 \mathrm{E}-02$ & $1.4 \mathrm{E}-02$ & $2.2 \mathrm{E}-02$ & $2.6 \mathrm{E}-02$ & 4.1E-02 \\
\hline 1 & 4.5E-03 & $6.4 \mathrm{E}-03$ & $4.7 \mathrm{E}-03$ & $6.5 \mathrm{E}-03$ & $1.2 \mathrm{E}-02$ & $1.6 \mathrm{E}-02$ & $2.1 \mathrm{E}-02$ & $2.9 \mathrm{E}-02$ & $3.8 \mathrm{E}-02$ & $5.2 \mathrm{E}-02$ \\
\hline 2 & $4.6 \mathrm{E}-03$ & $6.4 \mathrm{E}-03$ & $4.8 \mathrm{E}-03$ & $6.5 \mathrm{E}-03$ & $1.2 \mathrm{E}-02$ & $1.6 \mathrm{E}-02$ & $2.1 \mathrm{E}-02$ & $2.9 \mathrm{E}-02$ & $3.9 E-02$ & $5.3 \mathrm{E}-02$ \\
\hline $3-<6$ & $4.3 \mathrm{E}-03$ & $5.8 \mathrm{E}-03$ & $4.5 \mathrm{E}-03$ & $5.8 \mathrm{E}-03$ & $1.1 \mathrm{E}-02$ & $1.4 \mathrm{E}-02$ & $2.1 \mathrm{E}-02$ & $2.7 \mathrm{E}-02$ & $3.7 \mathrm{E}-02$ & $4.8 \mathrm{E}-02$ \\
\hline $6-<11$ & $4.5 \mathrm{E}-03$ & $6.3 \mathrm{E}-03$ & $4.8 \mathrm{E}-03$ & $6.4 \mathrm{E}-03$ & $1.1 \mathrm{E}-02$ & $1.5 \mathrm{E}-02$ & $2.2 \mathrm{E}-02$ & $2.9 \mathrm{E}-02$ & $4.2 \mathrm{E}-02$ & $5.9 \mathrm{E}-02$ \\
\hline $11-<16$ & $5.0 \mathrm{E}-03$ & 7.4E-03 & $5.4 \mathrm{E}-03$ & $7.5 \mathrm{E}-03$ & $1.3 \mathrm{E}-02$ & $1.7 \mathrm{E}-02$ & $2.5 \mathrm{E}-02$ & $3.4 \mathrm{E}-02$ & $4.9 \mathrm{E}-02$ & $7.0 \mathrm{E}-02$ \\
\hline $16-<21$ & $4.9 \mathrm{E}-03$ & $7.1 \mathrm{E}-03$ & $5.3 \mathrm{E}-03$ & $7.2 \mathrm{E}-03$ & $1.2 \mathrm{E}-02$ & $1.6 \mathrm{E}-02$ & $2.6 \mathrm{E}-02$ & $3.7 \mathrm{E}-02$ & $4.9 E-02$ & $7.3 \mathrm{E}-02$ \\
\hline $21-<31$ & $4.3 \mathrm{E}-03$ & $6.5 \mathrm{E}-03$ & $4.2 \mathrm{E}-03$ & $6.5 \mathrm{E}-03$ & $1.1 \mathrm{E}-02$ & $1.6 \mathrm{E}-02$ & $2.6 \mathrm{E}-02$ & $3.8 \mathrm{E}-02$ & $5.0 \mathrm{E}-02$ & $7.6 \mathrm{E}-02$ \\
\hline $41-<51$ & $5.0 \mathrm{E}-03$ & $7.1 \mathrm{E}-03$ & $4.8 \mathrm{E}-03$ & $7.0 \mathrm{E}-03$ & $1.2 \mathrm{E}-02$ & $1.6 \mathrm{E}-02$ & $2.8 \mathrm{E}-02$ & $3.9 \mathrm{E}-02$ & $5.2 \mathrm{E}-02$ & 7.6E-02 \\
\hline $51-<61$ & $5.2 \mathrm{E}-03$ & $7.5 \mathrm{E}-03$ & $5.0 \mathrm{E}-03$ & $7.3 \mathrm{E}-03$ & $1.2 \mathrm{E}-02$ & $1.7 \mathrm{E}-02$ & $2.9 \mathrm{E}-02$ & $4.0 \mathrm{E}-02$ & $5.3 \mathrm{E}-02$ & $7.8 \mathrm{E}-02$ \\
\hline $61-<71$ & $5.2 \mathrm{E}-03$ & $7.2 \mathrm{E}-03$ & $4.9 \mathrm{E}-03$ & $7.3 \mathrm{E}-03$ & $1.1 \mathrm{E}-02$ & $1.6 \mathrm{E}-02$ & $2.6 \mathrm{E}-02$ & $3.4 \mathrm{E}-02$ & $4.7 \mathrm{E}-02$ & $6.6 \mathrm{E}-02$ \\
\hline $71-<81$ & $5.3 \mathrm{E}-03$ & $7.2 \mathrm{E}-03$ & $5.0 \mathrm{E}-03$ & $7.2 \mathrm{E}-03$ & $1.1 \mathrm{E}-02$ & $1.5 \mathrm{E}-02$ & $2.5 \mathrm{E}-02$ & $3.2 \mathrm{E}-02$ & $4.7 \mathrm{E}-02$ & $6.5 \mathrm{E}-02$ \\
\hline$\geq 81$ & $5.2 \mathrm{E}-03$ & $7.0 \mathrm{E}-03$ & $4.9 \mathrm{E}-03$ & $7.0 \mathrm{E}-03$ & $1.1 \mathrm{E}-02$ & $1.6 \mathrm{E}-02$ & $2.5 \mathrm{E}-02$ & $3.1 \mathrm{E}-02$ & $4.8 \mathrm{E}-02$ & $6.8 \mathrm{E}-02$ \\
\hline
\end{tabular}

a) An individual's ventilation rate for the given activity category equals the weighted average of the individual's activity-specific ventilation rates for activities falling within the category, estimated using a multiple linear regression model, with weights corresponding to the number of minutes spent performing the activity. Numbers in these two columns represent averages, calculated across individuals in the specified age category, of these weighted averages. These are weighted averages, with the weights corresponding to the 4-year sampling weights assigned within NHANES 1999-2002.

Source: US-EPA, 2009, modified from US-EPA, 2009 
The US-EPA recommended values for inhalation rates presented in Table 4.5 and 4.6 for long-term and short-term exposure, respectively, are well validated as the general assessment factors (GAFs, described in Section 1.1) were used to judge the quality of the underlying data used to derive the recommendations. The US-EPA has assigned the following confidence ratings for the five GAFs: 1 . Soundness - medium, 2. applicability and utility - high, 3 . clarity and completeness - medium, 4 . variability and uncertainty - medium, and 5. evaluation and review - high; the overall rating was medium. (US-EPA, 2009).

\subsection{WHO}

WHO standard values for respiratory volumes (average figures) are those recommended by the ICRP (ICRP, 1974, cited in WHO/IPCS, 1994 and 1999). These values are presented in Table 4.7.

\begin{tabular}{ll} 
Table 4.7: WHO's Standard Values for Respiratory Volumes & \\
\hline Condition & Respiratory Volume (Average) \\
\hline Long-term exposure (8 hours resting, 16 hours light/non-occupational activity): & $15 \mathrm{~m}^{3} / \mathrm{day}^{3}$ \\
Child (10 Years) & $23 \mathrm{~m}^{3} / \mathrm{day}$ \\
Adult Man & $21 \mathrm{~m}^{3} / \mathrm{day}^{3}$ \\
Adult Woman & $22 \mathrm{~m}^{3} /$ day \\
Men and Women Combined & \\
Short-Time Exposure: & \\
Resting & $2300 \mathrm{~L} / 8$ hour \\
Child (10 Years) & $3600 \mathrm{~L} / 8$ hour \\
Adult Man & $2900 \mathrm{~L} / 8$ hour \\
Adult Woman & \\
Light/Nonoccupational Activity & $6240 \mathrm{~L} / 8$ hour \\
Child (10 Years) & $9600 \mathrm{~L} / 8$ hour \\
Adult Man & $9100 \mathrm{~L} / 8$ hour \\
Adult Woman & \\
\hline
\end{tabular}

Source: Modified from WHO/IPCS, 1994 and 1999

\subsection{Conclusion and Recommendations}

The data presented in the US-EPA EFH (US-EPA, 2009) are the most comprehensive. The European data are limited as are the WHO data.

In the REACH TGD Chapter R8 (ECHA, 2008a), the recommended values in Table R. 8-18 are stated as "taken from Gold et al., 1984 and ICRP, 1975"; however, it is not clear whether the recommended values are taken from one of these references or both; there are no references provided for the default physiological parameters presented in Table R. 8-2. In the REACH TGD Chapter R15 (ECHA, 2008b), the recommended values presented in Table 4.1 are taken from a German document (AUH, 1995).

The EFSA (EFSA, 2010) recommended values (Table 4.2) are based upon data from the 2009 version of the EFH (US-EPA, 2009). The Danish Environmental Protection Agency (D-EPA, 2006) has based their recom- 
mended values on the 1997 version of the EFH (US-EPA, 1997). The recommended values from ECETOC (ECETOC, 2001) presented in Table 4.3 and 4.4 for long-term and short-term exposure, respectively, are taken from the 1997 version of the EFH (US-EPA, 1997) as ECETOC has considered that these values are probably representative of Europeans as well.

The US-EPA recommended values for inhalation rates presented in Table 4.5 and 4.6 for long-term and short-term exposure, respectively, are well validated and valid as the general assessment factors (GAFs, described in Section 1.1) were used to judge the quality of the underlying data used to derive the recommendations and as the US-EPA overall confidence rating was medium. (US-EPA, 2009).

The WHO's standard values (Table 4.7) are based on recommendations from the ICRP (ICRP, 1974).

It should be noted that the references "ICRP, 1974" and "ICRP, 1975" are to the same ICRP report (ICRP Report No. 23).

Activity levels might be different among US individuals compared to Europeans. Despite this, the US-EPA recommended values are considered as being representative for Europeans as well. Furthermore, the US-EPA recommended values are considered as being the most valid values for inhalation rates as today as these recommendations are based on four recent key studies published in the period from 2006-2009. In addition, the US-EPA has noted that these key studies represent an improvement upon those previously used for recommended inhalation rates in previous versions of the EFH. In contrast, the European data are predominantly based on older references (ICRP, 1975; AUH 1995, USEPA, 1997) or no references have been provided for the recommended values (e.g., REACH TGD Chapter R8, Table R. 8-2). One European body, namely EFSA (EFSA; 2010) has based their recommended values on the most recent version of the EFH (US-EPA, 2009). It should also be noted that ECETOC has considered that the American values are probably representative of Europeans as well.

In conclusion, the US-EPA recommended values presented in Table 4.5 and 4.6 are considered as being the most valid values for inhalation rates as today and are therefore recommended as default exposure factors for assessments of the European population in the context of REACH.

The recommended values can also be used in the context of other chemical regulations such as those for e.g., biocides, pesticides, cosmetics, toys etc. 


\section{Drinking Water}

Drinking water is a potential source of human exposure to chemical substances, naturally occurring or contaminants. Contamination of drinking water may occur by, for example, percolation of chemicals through the soil to ground water that is used as a source of drinking water; runoff or discharge to surface water that is used as a source of drinking water, intentional or unintentional addition of substances to treat water (e.g., chlorination), and leaching of materials from plumbing systems (e.g., lead). The exposure is usually expressed as an average amount of drinking water consumed per unit time (e.g., litre/day).

For the estimation of the magnitude of the potential dose of chemicals from drinking water, information on the quantity of water consumed per unit time is required. The intake of drinking water depends on age, level of physical activity (working, running, walking, or resting), and the ambient temperature.

For the purposes of exposure assessments involving site-specific contaminated drinking water, ingestion rates based on the community supply are most appropriate. Given the assumption that bottled water, and purchased foods and beverages that contain water are widely distributed and less likely to contain source-specific water, the use of total water ingestion rates may overestimate the potential exposure to toxic substances present only in local water supplies (US-EPA, 2009); therefore, tap water ingestion rather than total water ingestion, is emphasized in this report.

\subsection{Europe}

In the REACH Guidance on information requirements and chemical safety assessment Chapter R8 (ECHA, 2008a) the standard value for daily water intake is 2.0 litres. This is also recommend by The EFSA Scientific Committee as a conservative default value for daily total liquid intake in adults (Draft Guidance on Default Assumptions 2011).

The Danish Environmental Protection Agency (D-EPA) has in their Guidance from the Environmental Protection Agency No. 5 (D-EPA, 2006) based their recommended intake of tap water upon data in the Exposure Factors Handbook (US-EPA, 1997). For newborns, the mean intake is 35 $\mathrm{ml} / \mathrm{kg}$ bw/day, while the $95^{\text {th }}$ percentile is $127 \mathrm{ml} / \mathrm{kg}$ bw/day. For children aged one to ten years, the mean intake is set to $31 \mathrm{ml} / \mathrm{kg} \mathrm{bw} /$ day and the $95^{\text {th }}$ percentile is $79.4 \mathrm{ml} / \mathrm{kg} \mathrm{bw} /$ day (D-EPA, 2006). It was considered most applicable to use the values for children (one to ten years) 
due to possible differences in breastfeeding patterns in Denmark and USA. These differences across countries may be important for the intake of tap water for newborns (D-EPA, 2006).

In the Exposure Factors Sourcebook for European Populations, with Focus on UK Data (ECETOC, 2001) an average value of 1.1 litre/day for adults and 0.5 litre/day for 1-11 year olds is recommended based upon a 1980 survey of Great Britain (Hopkin and Ellis, 1980, as cited in the 1997 version of the EFH (US-EPA, 1997)), see Table 5.1. This survey, included in the 2009 version of the EFH (US-EPA, 2009) as a "relevant study" was based on questionnaires and diaries indicating the type and quantity of beverages consumed over a one-week period. In the survey, drinking water is estimated as total tap water ingestion, which includes tap water consumed directly and in prepared items such as tea, coffee and other drinks, excludes water ingestion from bottled beverages. Because bottled items are likely to originate from different water sources (US-EPA, 1997), total tap water ingestion is therefore, according to ECETOC a better estimation of intake from the greatest single water source. (ECETOC, 2001).

$\begin{aligned} & \text { Table 5.1: Summary of Total Tap water Intake for Men and Women (litre/day) - as Presented in } \\
& \text { the Exposure Factors Sourcebook for European Populations, with Focus on UK Data }\end{aligned}$
\begin{tabular}{lcccc}
\hline Age Group (years) & \multicolumn{2}{l}{ Mean Intake (litre/day) } & 10 and $\mathbf{9 0}$ Percentiles (litre/day) \\
\cline { 2 - 5 } & Men & Women & Men & Women \\
\hline $1-4$ & 0.477 & 0.464 & $0.17-0.85$ & $0.15-0.89$ \\
$5-11$ & 0.550 & 0.533 & $0.22-0.90$ & $0.22-0.93$ \\
$12-17$ & 0.805 & 0.725 & $0.29-1.35$ & $0.31-1.16$ \\
$18-30$ & 1.006 & 0.991 & $0.45-1.62$ & $0.50-1.55$ \\
$31-54$ & 1.201 & 1.091 & $0.64-1.88$ & $0.62-1.68$ \\
$55+$ & 1.133 & 1.027 & $0.62-1.72$ & $0.54-1.57$ \\
\hline
\end{tabular}

Source: US-EPA (1997) citation of Hopkin and Ellis, 1980, modified from ECETOC, 2001

\subsection{US-EPA}

In the Exposure Factors Handbook (EFH) (US-EPA, 2009) consumption of food and water is divided into "per capita" and "consumers only." Per capita intake rate is "the average quantity of food consumed per person in a population composed of both individuals who ate the food during a specified time period and those that did not." Consumer-only intake rate on the other hand, is "the average quantity of food consumed per person in a population composed only of individuals who ate the food item of interest during a specified period."

Historically, the US-EPA has assumed a drinking water ingestion rate of $2 \mathrm{~L}$ per day for adults and $1 \mathrm{~L}$ per day for infants and children less than 10 years of age (US-EPA, 2000). This rate includes water consumed in the form of juices and other beverages containing tap water.

In the EFH, two "key studies" and 22 "relevant studies" were identified. The key studies (Kahn, 2008 and Kahn \& Stralka, 2008a) were iden- 
tified based on the applicability of the survey design to exposure assessments of the entire US population. A total of approximately 20,000 individuals were included in the surveys. The data were collected by an in-home interviewer on a recall basis on two non-consecutive days within ten days using standardized volumes of drinking water containers. The recommended exposure factors for drinking water - based on the two key studies - are presented in Table 5.2. A third (Kahn \& Stralka, $2008 \mathrm{~b}$ ) forms the basis for the recommended exposure factors for pregnant and lactating women.

The recommended values presented in Table 5.2 are well validated as the general assessment factors (GAFs, described in Section 2.1) were used to judge the quality of the underlying data used to derive the recommendations. For children and adults, the US-EPA has assigned the following confidence ratings for the five GAFs: 1 . Soundness - medium to high, 2. applicability and utility - medium, 3. clarity and completeness high, 4. variability and uncertainty - high, and 5. evaluation and review medium; the overall rating was medium to high. For pregnant/lactating women, the US-EPA has assigned the following confidence ratings for the five GAFs: 1 . Soundness - low, 2. applicability and utility - lowmedium, 3. clarity and completeness - medium, 4. variability and uncertainty - low, and 5. evaluation and review - medium; the overall rating was low. (US-EPA, 2009).

One of the relevant studies (Heller et al., 2000, cited from US-EPA, 2009) provides information on tap water and total water consumption by age and sex using the same survey as the two key studies, see Table D.1 in Annex D.

\begin{tabular}{|c|c|c|c|c|}
\hline Age group & $\begin{array}{r}\text { Mean } \\
\text { (ml/day) }\end{array}$ & $\begin{array}{r}\text { Mean } \\
(\mathrm{ml} / \mathrm{kg} \text { bw/day) }\end{array}$ & $\begin{array}{r}95^{\text {th }} \text { Percentile } \\
(\mathrm{ml} / \text { day })\end{array}$ & $\begin{array}{r}95^{\text {th }} \text { Percentile } \\
\text { (ml/kg bw/day) }\end{array}$ \\
\hline \multicolumn{5}{|l|}{ Per Capita } \\
\hline \multicolumn{5}{|l|}{ Children } \\
\hline Birth to $<1$ month & 184 & 52 & 839 & 232 \\
\hline 1 to $<3$ month & 227 & 48 & 896 & 205 \\
\hline 3 to $<6$ month & 362 & 52 & 1,056 & 159 \\
\hline 6 to $<12$ month & 360 & 41 & 1,055 & 126 \\
\hline 1 to $<2$ years & 271 & 23 & 837 & 71 \\
\hline 2 to $<3$ years & 317 & 23 & 877 & 60 \\
\hline 3 to $<6$ years & 380 & 22 & 1,078 & 61 \\
\hline 6 to $<11$ years & 447 & 16 & 1,235 & 43 \\
\hline 11 to $<16$ years & 606 & 12 & 1,727 & 34 \\
\hline 16 to $<18$ years & 731 & 11 & 1,983 & 31 \\
\hline 18 to $<21$ years & 826 & 12 & 2,540 & 35 \\
\hline \multicolumn{5}{|l|}{ Adults } \\
\hline$\geq 21$ years & 1,104 & 15 & 2,811 & 39 \\
\hline$>65$ years & 1,127 & 15 & 2,551 & 37 \\
\hline All ages & 926 & 16 & 2,544 & 43 \\
\hline Pregnant women & 819 & 13 & 2,503 & 43 \\
\hline Lactating women & 1,379 & 21 & 3,434 & 55 \\
\hline
\end{tabular}




\begin{tabular}{lrrrr}
\hline Age group & $\begin{array}{r}\text { Mean } \\
\text { (ml/day) }\end{array}$ & $\begin{array}{r}\text { Mean } \\
\text { (ml/kg bw/day) }\end{array}$ & $\begin{array}{r}\mathbf{9 5}^{\text {th }} \text { Percentile } \\
\text { (ml/day) }\end{array}$ & $\begin{array}{r}\mathbf{9 5}^{\text {th }} \text { Percentile } \\
\text { (ml/kg bw/day) }\end{array}$ \\
\hline Children & & & & 238 \\
Birth to $<1$ month & 470 & 137 & 858 & 285 \\
1 to $<3$ month & 552 & 119 & 1,053 & 173 \\
3 to $<6$ month & 556 & 80 & 1,171 & 129 \\
6 to $<12$ month & 467 & 53 & 1,147 & 75 \\
1 to $<2$ years & 308 & 27 & 893 & 62 \\
2 to $<3$ years & 356 & 26 & 912 & 65 \\
3 to $<6$ years & 417 & 24 & 1,099 & 45 \\
6 to $<11$ years & 480 & 17 & 1,251 & 34 \\
11 to $<16$ years & 652 & 13 & 1,744 & 32 \\
16 to $<18$ years & 792 & 12 & 2,002 & 35 \\
18 to $<21$ years & 895 & 13 & 2,565 & \\
Adults & & & & 39 \\
$\geq 21$ years & 1,183 & 16 & 2,848 & 37 \\
$>65$ years & 1,242 & 18 & 2,604 & 43 \\
All ages & 1,000 & 17 & 2,601 & 43 \\
Pregnant women & 872 & 14 & 2,589 & 55 \\
Lactating women & 1,665 & 26 & 3,588 & \\
\hline
\end{tabular}

a) Ingestion rates for combined direct (direct consumption of water as a beverage) and indirect (includes water added during food preparation, but not water intrinsic to purchased foods (i.e. water that is naturally contained in foods)) water from community water supply.

Source: Kahn, 2008 and Kahn \& Stralka, 2008a, b, modified from US-EPA, 2009.

\subsection{WHO}

Table 5.3 presents WHO's standard values of daily fluid intake for different conditions based on recommendations from the ICRP (ICRP, 1974 cited in WHO/IPCS, 1994 and 1999).

In developing drinking water guideline values for potentially hazardous chemicals, the WHO (WHO, 2008) assumes a daily per capita consumption of 2 litres of drinking water by a person weighing $60 \mathrm{~kg}$. This standard value is considered to be on the safe side in most situations. But under certain circumstances, this assumption may underestimate the consumption of water per unit weight, and thus exposure, for those living in hot climates as well as for infants and children, who consume more fluid per unit weight than adults. Where it was judged that children were at a particularly high risk from exposure to certain chemicals, the drinking water guideline value was derived on the basis of a $10-\mathrm{kg}$ child consuming 1 litre of drinking water per day, or a 5-kg infant consuming 0.75 litre per day. The corresponding daily fluid intakes are higher than for adults on a body weight basis (WHO, 2008). 
Table 5.3: WHO's Standard Values for Daily Fluid Intake

\begin{tabular}{lr}
\hline Condition & Daily Fluid Intake (ml/day) (milk, tap water, other beverages) \\
\hline Normal Conditions & $1000-2400$, representative figure $=1900^{\text {a }}\left(\right.$ excluding milk: $\left.1400^{\mathrm{b}}\right)$ \\
Adults & 1950 \\
Adult Men & 1400 \\
Adult Women & 1400 \\
Child $(10$ years) & \\
High Average Temperature $\left(\mathbf{3 2}^{\circ} \mathbf{C}\right)$ & $2840-3410$ \\
Adults & 3700 \\
Moderate Activity & \\
Adults & \\
\hline
\end{tabular}

a) WHO uses a daily per capita drinking-water consumption of 2 litres in calculating water quality guidelines.

b) From Health and Welfare Canada, 1992.

Source: Modified from WHO/IPCS, 1994 and 1999.

\subsection{Conclusion and Recommendations}

The data presented in the US-EPA EFH (US-EPA, 2009) are the most comprehensive. The European data are limited as are the WHO data.

In the REACH TGD Chapter R8 (ECHA, 2008a), the recommended values are stated as "taken from Gold et al., 1984 and ICRP, 1975"; however, it is not clear whether the recommendations are taken from one of these references or both. The Danish Environmental Protection Agency (DEPA, 2006) has based their recommendations on the 1997 version of the EFH (US-EPA, 1997).

The recommended values from ECETOC (ECETOC, 2001) presented in Table 5.1 are based upon a thirty-year old survey as it has been described in the 1997 version of the EFH (US-EPA, 1997). This survey is included in the 2009 version of the EFH (US-EPA, 2009) as a "relevant study" and the US-EPA has evaluated that the advantage of the data in this survey is that the responses were not generated on a recall basis, but by recording daily intake in diaries, which may result in more accurate responses being generated. But as the data were based on the population of Great Britain and not the United States and as drinking patterns may differ among these populations as a result of varying weather conditions and socio-economic factors, this survey was not considered as a "key study" in the 2009 version of the EFH.

The US-EPA recommended values for intake of drinking water presented in Table 5.2 are well validated and valid as the general assessment factors (GAFs, described in Section 2.1) were used to judge the quality of the underlying data used to derive the recommendations. For children and adults, the US-EPA overall rating was medium to high. For pregnant/lactating women, the US-EPA overall rating was low. (US-EPA, 2009).

The WHO's standard values of daily fluid intake (Table 5.3) are based on recommendations from the ICRP (ICRP, 1974). 
It should be noted that the references "ICRP, 1974" and "ICRP, 1975" are to the same ICRP report (ICRP Report No. 23).

The intake of bottled beverages may be more common today than it was thirty years ago. Therefore, the more recent recommendations from the US-EPA may be more representative of the intake of tap water as today compared to the recommended values from ECETOC, which have been based upon a thirty-year old survey as it has been described in the 1997 version of the EFH (US-EPA, 1997). However, it is possible that the US population consumes more bottled beverages than the European population and that the US data therefore underestimate the actual intake of tap water consumed by the European population. Despite this, the US-EPA recommended values are considered as being representative for Europeans as well. Furthermore, the US-EPA recommended values are considered as being the most valid values for intake of drinking water as today as these recommendations are based on two very recent key studies published in 2008. In contrast, the European data are predominantly based on older references (ICRP, 1975; US-EPA, 1997).

In conclusion, the US-EPA recommended values presented in Table 5.2 are considered as being the most valid values for intake of drinking water as today and are therefore recommended as default exposure factors for assessments of the European population in the context of REACH.

The recommended values can also be used in the context of other chemical regulations such as those for e.g., biocides, pesticides etc. 


\section{Food Intake}

Even though food supply in the western world is generally considered to be safe, contamination of foods may occur as a result of environmental pollution of the air, water, or soil, or the intentional use of chemicals such as pesticides or other agrochemicals. Ingestion of contaminated foods is a potential pathway of exposure to such contaminants. To assess chemical exposure through this pathway, information on food ingestion rates is needed. (US-EPA, 2009).

\subsection{Europe}

The standard value for total food intake in the REACH Guidance on information requirements and chemical safety assessment Chapter R8 (EC$\mathrm{HA}, 2008 \mathrm{a}$ ) is appointed to $1.4 \mathrm{~kg}$ per day.

The National Food Institute, Technical University of Denmark, has investigated the dietary habits of the Danish population in a document called Dietary habits in Denmark 2003-2008 - Main Results (DTUF00D, 2010) (Danskernes Kostvaner 2003-2008 - Hovedresultater). In this survey, dietary data from 4431 individuals aged 4-75 years has been collected through a seven day diary between 2003 and 2008. Table 6.1 presents main results from the Danish survey and Table E.1 in Annex E presents the detailed results.

\begin{tabular}{lcc} 
Table 6.1: Dietary Habits in Denmark 2003-2008 & & \\
\hline Group (years) & Mean & $\mathbf{9 0}^{\text {th }}$ percentiles \\
\hline Cheese and cheese products (g/day) & 19 & 37 \\
All children 4-9 & 26 & 52 \\
All children 10-17 & 34 & 65 \\
All adults 18-75 & 31 & 62 \\
Total (all) & & 276 \\
Cereals including bread (g/day) & 199 & 298 \\
All children 4-9 & 212 & 320 \\
All children 10-17 & 214 & 314 \\
All adults 18-75 & 212 & 207 \\
Total (all) & & 230 \\
Vegetables exclusive potatoes (g/day) & 122 & 278 \\
All children 4-9 & 131 & 265 \\
All children 10-17 & 162 & \\
All adults 18-75 & 153 & 139 \\
Total (all) & & 198 \\
Potatoes and potato products (g/day) & 74 & 219 \\
All children 4-9 & 95 & 189 \\
All children 10-17 & 112 & \\
All adults 18-75 & 94 & \\
Total (all) & &
\end{tabular}




\begin{tabular}{|c|c|c|}
\hline Group (years) & Mean & $90^{\text {th }}$ percentiles \\
\hline \multicolumn{3}{|c|}{ Fruits and fruit products (g/day) } \\
\hline All children 4-9 & 260 & 469 \\
\hline All children $10-17$ & 274 & 541 \\
\hline All adults $18-75$ & 283 & 548 \\
\hline Total (all) & 280 & 541 \\
\hline \multicolumn{3}{|c|}{ Meat and meat products (g/day) } \\
\hline All children 4-9 & 83 & 126 \\
\hline All children $10-17$ & 97 & 162 \\
\hline All adults $18-75$ & 109 & 188 \\
\hline Total (all) & 105 & 179 \\
\hline \multicolumn{3}{|c|}{ Poultry and poultry products (g/day) } \\
\hline All children 4-9 & 16 & 37 \\
\hline All children $10-17$ & 22 & 51 \\
\hline All adults $18-75$ & 23 & 55 \\
\hline Total (all) & 22 & 53 \\
\hline \multicolumn{3}{|c|}{ Fish and fish products (g/day) } \\
\hline All children 4-9 & 12 & 31 \\
\hline All children 10-17 & 11 & 28 \\
\hline All adults $18-75$ & 22 & 47 \\
\hline Total (all) & 19 & 44 \\
\hline \multicolumn{3}{|l|}{ Eggs (g/day) } \\
\hline All children 4-9 & 13 & 26 \\
\hline All children 10-17 & 12 & 28 \\
\hline All adults $18-75$ & 17 & 35 \\
\hline Total (all) & 16 & 33 \\
\hline \multicolumn{3}{|l|}{ Fats (g/day) } \\
\hline All children 4-9 & 33 & 55 \\
\hline All children $10-17$ & 30 & 52 \\
\hline All adults $18-75$ & 35 & 63 \\
\hline Total (all) & 34 & 60 \\
\hline \multicolumn{3}{|c|}{ Sugar and candy (g/day) } \\
\hline All children 4-9 & 39 & 67 \\
\hline All children 10-17 & 42 & 78 \\
\hline All adults $18-75$ & 34 & 69 \\
\hline Total (all) & 36 & 70 \\
\hline \multicolumn{3}{|l|}{ Energy (MJ/day) } \\
\hline All children 4-9 & 8.0 & 10.6 \\
\hline All children 10-17 & 8.6 & 11.6 \\
\hline All adults $18-75$ & 9.1 & 12.9 \\
\hline Total (all) & 8.9 & 12.5 \\
\hline
\end{tabular}

Source: Modified from Dietary habits in Denmark 2003-2008 - Main Results, National Food Institute, Technical University of Denmark, (DTU-FOOD, 2010)

The recommended food consumption rates from the Exposure Factors Sourcebook for European Populations, with Focus on UK Data (ECETOC, 2001) are presented in Table 6.2. 
Table 6.2: Recommendations on the Average Food Consumption in the European Community and its Member Countries - as Presented in the Exposure Factors Sourcebook for European Populations, with Focus on UK Data

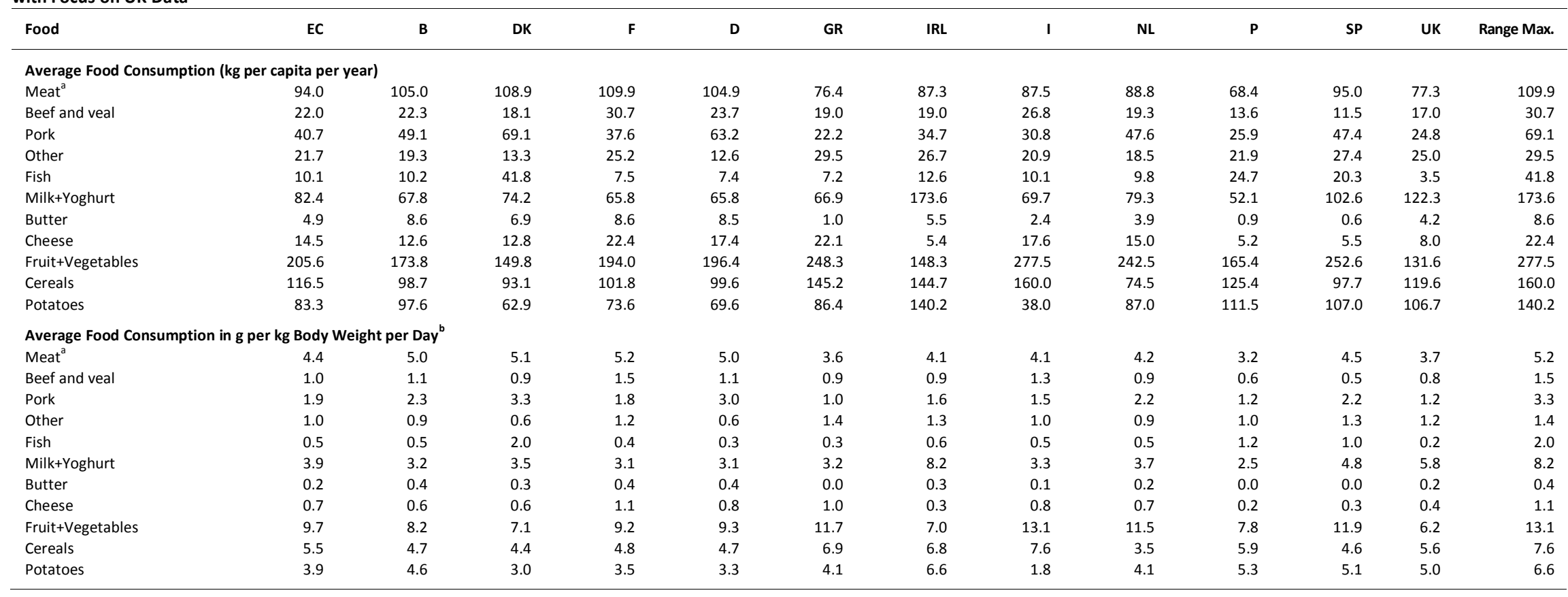

a) Total does not reflect the sum of the subgroups.

b) Averages over lifetime assuming average bodyweight of $58 \mathrm{~kg}$ (Table 1 values divided by $58 \mathrm{~kg}$ and 365 days/year).

Source: ECETOC, 1994 citation of Euromonitor 1992, European Marketing Data and Statistics, modified from ECETOC, 2001 
According to the Exposure Factors Sourcebook for European Populations, with Focus on UK Data (ECETOC, 2001), the recommendations for breast milk intake are the same as presented in the 1997 version of the Exposure Factors Handbook (US-EPA, 1997). No UK studies were cited in USEPA (1997), but breast milk consumption values for Swedish infants were similar to those of US infants. The data from US-EPA (1997), is presented in Table 6.3.

Table 6.3: Standard Breast Milk Intake Rates - as Presented in the Exposure Factors Sourcebook for European Populations, with Focus on UK Data

\begin{tabular}{lrr}
\hline Age & Mean (ml/day) & Upper percentile (mean + 2 SD) \\
\hline $1-6$ months & 742 & 1033 \\
12 months & 688 & 980 \\
\hline
\end{tabular}

Source: US-EPA, 1997, modified from ECETOC, 2001.

\subsection{US-EPA}

In the Exposure Factors Handbook (US-EPA, 2009), recommendations on intake of food are divided into different food categories: Fruits and Vegetables, Fish, Meats, Dairy and Fats, Grains, Total food intake, and Human Breast Milk and Lipids.

\subsubsection{Fruits and Vegetables}

The US-EPA analysis of data from the 1994-96 and 1998 Continuing Survey of Food Intake by Individuals (CSFII) was used in selecting recommended intake rates for the general population and children in the Exposure Factors Handbook (US-EPA, 2009). Table 6.4 presents a summary of the recommended values for per capita and consumer-only intake of fruits and vegetables, on an as-consumed basis. Total fruit intake refers to the sum of all fruits consumed in a day including canned, dried, frozen, and fresh fruits. Likewise, total vegetable intake refers to the sum of all vegetables consumed in a day including canned, dried, frozen, and fresh vegetables.

The US-EPA recommended values presented in Table 6.4 are well validated as the general assessment factors (GAFs, described in Section 1.1) were used to judge the quality of the underlying data used to derive the recommendations. The US-EPA has assigned the following confidence ratings for the five GAFs: 1 . Soundness - high, 2. applicability and utility - medium, 3. clarity and completeness - high, 4 . variability and uncertainty - medium, and 5. evaluation and review - medium; the overall rating was medium-high. (US-EPA, 2009). 
Table 6.4: Recommended Values for Intake of Fruits and Vegetables, As Consumed - as Presented in the Exposure Factors Handbook

\begin{tabular}{|c|c|c|c|c|c|c|c|c|}
\hline \multirow[t]{3}{*}{ Age (Years) } & \multicolumn{4}{|c|}{ Total Fruits (g/kg-day) } & \multicolumn{4}{|c|}{ Total Vegetables (g/kg-day) } \\
\hline & \multicolumn{2}{|c|}{ Per Capita } & \multicolumn{2}{|c|}{ Consumers Only } & \multicolumn{2}{|c|}{ Per Capita } & \multicolumn{2}{|c|}{ Consumers Only } \\
\hline & Mean & $95^{\text {th }}$ & Mean & $95^{\text {th }}$ & Mean & $95^{\text {th }}$ & Mean & $95^{\text {th }}$ \\
\hline $0-1$ & 5.7 & 21.3 & 10.1 & 26.4 & 4.5 & 14.8 & 6.2 & 16.1 \\
\hline $1-<2$ & 6.2 & 18.5 & 6.9 & 19.0 & 6.9 & 17.1 & 6.9 & 17.1 \\
\hline $2-<3$ & 6.2 & 18.5 & 6.9 & 19.0 & 6.9 & 17.1 & 6.9 & 17.1 \\
\hline $3-<6$ & 4.6 & 14.4 & 5.1 & 15.0 & 5.9 & 14.7 & 5.9 & 14.7 \\
\hline $6-<11$ & 2.4 & 8.8 & 2.7 & 9.3 & 4.1 & 9.9 & 4.1 & 9.9 \\
\hline $11-<16$ & 0.8 & 3.5 & 1.1 & 3.7 & 2.9 & 6.9 & 2.9 & 6.9 \\
\hline $16-<21$ & 0.8 & 3.5 & 1.1 & 3.7 & 2.9 & 6.9 & 2.9 & 6.9 \\
\hline $20-<50$ & 0.9 & 3.9 & 1.2 & 4.4 & 2.9 & 6.8 & 2.9 & 6.8 \\
\hline$\geq 50$ & 1.4 & 4.8 & 1.6 & 5.0 & 3.1 & 7.0 & 3.1 & 7.0 \\
\hline
\end{tabular}

Source: US-EPA Analysis of CSFII, 1994-96 and 1998, based on USDA, 2000 and US-EPA, 2000, modified from US-EPA, 2009

\subsubsection{Fish}

Due to considerable variation in the studies presented in the Exposure Factors Handbook (US-EPA, 2009), fish consumption studies were classified into the following categories:

- General Population (total, marine, freshwater/estuarine)

- Recreational Marine Intake

The key study identified in the Exposure Factors Handbook (US-EPA, 2009) for mean fish intake among the general population is based on a US-EPA (2002) analysis of data from the US Department of Agriculture (USDA) Continuing Survey of Food Intake among Individuals (CSFII) 1994-1996, 1998 (USDA, 2000). Data from this key study is presented in Table 6.5.

The US-EPA recommended values presented in Table 6.5 are well validated as the general assessment factors (GAFs, described in Section 1.1) were used to judge the quality of the underlying data used to derive the recommendations. The US-EPA has assigned the following confidence ratings for the five GAFs: 1 . Soundness - medium, 2. applicability and utility - medium, 3. clarity and completeness - high, 4. variability and uncertainty - medium, and 5. evaluation and review - medium; the overall rating was medium. (US-EPA, 2009). 
Table 6.5: Recommended Values for General Population and Recreational Population Fish Intake as Presented in the Exposure Factors Handbook

\begin{tabular}{|c|c|c|c|c|c|c|c|c|}
\hline \multirow[t]{3}{*}{ Age (Years) } & \multicolumn{4}{|c|}{ Per Capita } & \multicolumn{4}{|c|}{ Consumers Only } \\
\hline & \multicolumn{2}{|c|}{ Mean } & \multicolumn{2}{|c|}{$95^{\text {th }}$ Percentile } & \multicolumn{2}{|c|}{ Mean } & \multicolumn{2}{|c|}{$95^{\text {th }}$ Percentile } \\
\hline & g/day & $\begin{array}{l}\text { g/kg- } \\
\text { day }\end{array}$ & g/day & $\begin{array}{l}\text { g/kg- } \\
\text { day }\end{array}$ & g/day & $\begin{array}{l}\text { g/kg- } \\
\text { day }\end{array}$ & g/day & $\begin{array}{l}\text { g/kg- } \\
\text { day }\end{array}$ \\
\hline \multicolumn{9}{|c|}{ General Population - Total Fish ${ }^{\mathrm{a}}$} \\
\hline $3-<6$ & 7.7 & 0.43 & 51.0 & 3.0 & 74 & 4.2 & 184 & 10 \\
\hline $6-<11$ & 8.5 & 0.28 & 56.4 & 1.9 & 95 & 3.2 & 313 & 8.7 \\
\hline $11-<16$ & 12.0 & 0.23 & 87.4 & 1.5 & 113 & 2.2 & 308 & 6.2 \\
\hline $16-<18$ & 10.6 & 0.16 & 83.5 & 1.3 & 136 & 2.1 & 357 & 6.6 \\
\hline$>18$ & 19.9 & 0.27 & 111.3 & 1.5 & 127 & 1.8 & 334 & 4.5 \\
\hline \multicolumn{9}{|c|}{ General Population - Marine Fish ${ }^{a}$} \\
\hline $3-<6$ & 5.5 & 0.31 & 39.4 & 2.3 & 66 & 3.7 & 165 & 9.3 \\
\hline $6-<11$ & 5.6 & 0.20 & 38.4 & 1.5 & 78 & 2.8 & 202 & 8.0 \\
\hline $11-<16$ & 7.6 & 0.15 & 56.5 & 1.3 & 102 & 2.0 & 262 & 5.2 \\
\hline $16-<18$ & 6.1 & 0.10 & 29.5 & 0.5 & 126 & 2.0 & 353 & 6.5 \\
\hline$>18$ & 12.4 & 0.17 & 80.7 & 1.1 & 108 & 1.5 & 270 & 3.7 \\
\hline \multicolumn{9}{|c|}{ General Population - Freshwater/Estuarine Fish ${ }^{\mathrm{a}}$} \\
\hline $3-<6$ & 2.2 & 0.12 & 12.2 & 0.7 & 40 & 2.3 & 129 & 7.2 \\
\hline $6-<11$ & 3.0 & 0.08 & 13.1 & 0.4 & 61 & 1.8 & 248 & 6.2 \\
\hline $11-<16$ & 4.3 & 0.08 & 25.8 & 0.5 & 71 & 1.3 & 199 & 4.4 \\
\hline $16-<18$ & 4.6 & 0.07 & 19.3 & 0.3 & 100 & 1.4 & 242 & 3.3 \\
\hline$>18$ & 7.5 & 0.10 & 49.6 & 0.7 & 81 & 1.1 & 279 & 3.7 \\
\hline \multicolumn{9}{|c|}{ Recreational Population - Marine Fish - Atlantic } \\
\hline $3-<6$ & 2.5 & & 8.2 & & & & & \\
\hline $6-<11$ & 2.5 & & 9.1 & & & & & \\
\hline $11-<16$ & 3.4 & & 14.1 & & & & & \\
\hline $16-<18$ & 2.8 & & 13.5 & & & & & \\
\hline$>18$ & 5.6 & & 18.0 & & & & & \\
\hline \multicolumn{9}{|c|}{ Recreational Population - Marine Fish - Gulf } \\
\hline $3-<6$ & 3.2 & & 12.0 & & & & & \\
\hline $6-<11$ & 3.3 & & 13.2 & & & & & \\
\hline $11-<16$ & 4.4 & & 20.5 & & & & & \\
\hline $16-<18$ & 3.5 & & 19.6 & & & & & \\
\hline$>18$ & 7.2 & & 26.1 & & & & & \\
\hline \multicolumn{9}{|c|}{ Recreational Population - Marine Fish - Pacific } \\
\hline $3-<6$ & 0.9 & & 3.1 & & & & & \\
\hline $6-<11$ & 0.9 & & 3.4 & & & & & \\
\hline $11-<16$ & 1.2 & & 5.3 & & & & & \\
\hline $16-<18$ & 1.0 & & 5.1 & & & & & \\
\hline$>18$ & 2.0 & & 6.8 & & & & & \\
\hline
\end{tabular}

a) Rates are for uncooked fish.

Source: US-EPA, 2002 and US-EPA analysis of NMFS, 1993, modified from US-EPA, 2009.

\subsubsection{Meats, Dairy Products and Fats}

In the Exposure Factors Handbook (US-EPA, 2009), US-EPA analyses of data from the 1994-96 and 1998 CSFII (USDA, 2000) were used in selecting recommended intake rates for the general population. Table 6.6 presents a summary of the recommended values for per capita and consumers-only intake of meats, dairy products, and fats, on an asconsumed basis.

The US-EPA recommended values presented in Table 6.6 are well validated as the general assessment factors (GAFs, described in Section 1.1) were used to judge the quality of the underlying data used to derive the recommendations. The US-EPA has assigned the following confidence 
ratings for the five GAFs: 1 . Soundness - high, 2. applicability and utility medium, 3. clarity and completeness - high, 4. variability and uncertainty - medium, and 5. evaluation and review - medium; the overall rating was high. (US-EPA, 2009).

\begin{tabular}{|c|c|c|c|c|c|c|c|c|c|c|c|c|}
\hline \multirow[t]{3}{*}{ Age } & \multicolumn{4}{|c|}{ Total Meats (g/kg-day) } & \multicolumn{4}{|c|}{ Total Dairy Products (g/kg-day) } & \multicolumn{4}{|c|}{ Total Fats (g/kg-day) } \\
\hline & \multicolumn{2}{|c|}{ Per Capita } & \multicolumn{2}{|c|}{ Consumers } & \multicolumn{2}{|c|}{ Per Capita } & \multicolumn{2}{|c|}{ Consumers } & \multicolumn{2}{|c|}{ Per Capita } & \multicolumn{2}{|c|}{ Consumers } \\
\hline & Mean & $95^{\text {th }}$ & Mean & $95^{\text {th }}$ & Mean & $95^{\text {th }}$ & Mean & $95^{\text {th }}$ & Mean & $95^{\text {th }}$ & Mean & $95^{\text {th }}$ \\
\hline $0-<1 \mathrm{~m}$ & & & & & & & & & 5.2 & 16 & 7.8 & 16 \\
\hline $1-<3 m$ & & & & & & & & & 4.5 & 11 & 6.0 & 12 \\
\hline $3-6<m$ & & & & & & & & & 4.1 & 8.2 & 4.4 & 8.3 \\
\hline $6-12<m$ & & & & & & & & & 3.7 & 7.0 & 3.7 & 7.0 \\
\hline $0-1$ y & 1.2 & 6.7 & 3.0 & 9.2 & 12.6 & 48.7 & 15.9 & 57.5 & & & & \\
\hline $1-<2 y$ & 4.1 & 9.8 & 4.2 & 9.8 & 36.7 & 88.3 & 36.8 & 88.3 & 4.0 & 7.1 & 4.0 & 7.1 \\
\hline $2-<3 y$ & 4.1 & 9.8 & 4.2 & 9.8 & 36.7 & 88.3 & 36.8 & 88.3 & 3.6 & 6.4 & 3.6 & 6.4 \\
\hline $3-<6 y$ & 4.1 & 9.4 & 4.2 & 9.4 & 23.3 & 49.4 & 23.3 & 49.4 & 3.4 & 5.8 & 3.4 & 5.8 \\
\hline $6-<11 y$ & 2.9 & 6.5 & 2.9 & 6.5 & 13.6 & 31.5 & 13.6 & 31.5 & 2.6 & 4.2 & 2.6 & 4.2 \\
\hline $11-<16 y$ & 2.1 & 4.8 & 2.1 & 4.8 & 5.6 & 15.5 & 5.6 & 15.5 & 1.6 & 3.0 & 1.6 & 3.0 \\
\hline $16-<21 y$ & 2.1 & 4.8 & 2.1 & 4.8 & 5.6 & 15.5 & 5.6 & 15.5 & 1.3 & 2.7 & 1.3 & 2.7 \\
\hline $21-<31$ y & & & & & & & & & 1.2 & 2.3 & 1.2 & 2.3 \\
\hline $31-<41$ y & & & & & & & & & 1.1 & 2.1 & 1.1 & 2.1 \\
\hline $41-<51$ y & & & & & & & & & 1.0 & 1.9 & 1.0 & 1.9 \\
\hline $20-<50 y$ & 1.9 & 4.2 & 1.9 & 4.2 & 3.3 & 9.9 & 3.3 & 9.9 & & & & \\
\hline$\geq 50 y$ & 1.5 & 3.3 & 1.5 & 3.3 & 3.2 & 8.9 & 3.2 & 8.9 & & & & \\
\hline $51-<61$ y & & & & & & & & & 0.9 & 1.7 & 0.9 & 1.7 \\
\hline $61-<71$ y & & & & & & & & & 0.9 & 1.7 & 0.9 & 1.7 \\
\hline $71-<81$ y & & & & & & & & & 0.8 & 1.5 & 0.8 & 1.5 \\
\hline$\geq 81 \mathrm{y}$ & & & & & & & & & 0.9 & 1.5 & 0.9 & 1.5 \\
\hline
\end{tabular}

Source: US-EPA analysis of CSFII, 1994-96 and 1998, based on USDA, 2000 and US-EPA, 2000 and 2007b, modified from US-EPA, 2009.

\subsubsection{Grains}

The US-EPA analyses of data from the 1994-96 and 1998 CSFII were used in selecting recommended intake rates of grains for the general population in the Exposure Factors Handbook (US-EPA, 2009). Table 6.7 presents a summary of the recommended values for per capita and consumer-only intake of grain products, on an as-consumed basis.

The US-EPA recommended values presented in Table 6.7 are well validated as the general assessment factors (GAFs, described in Section 1.1) were used to judge the quality of the underlying data used to derive the recommendations. The US-EPA has assigned the following confidence ratings for the five GAFs: 1 . Soundness - high, 2. applicability and utility - medium, 3. clarity and completeness - high, 4. variability and uncertainty - medium, and 5. evaluation and review - medium; the overall rating was medium-high. (US-EPA, 2009). 
Table 6.7: Recommended Values for Intake of Grains, As Consumed - as Presented in the Exposure Factors Handbook

\begin{tabular}{|c|c|c|c|c|}
\hline \multirow[t]{2}{*}{ Age (year) } & \multicolumn{2}{|c|}{ Per Capita } & \multicolumn{2}{|c|}{ Consumers Only } \\
\hline & Mean (g/kg-day) & $95^{\text {th }}$ (g/kg-day) & Mean (g/kg-day) & $95^{\text {th }}$ (g/kg-day) \\
\hline $0-1$ & 2.5 & 8.6 & 3.6 & 9.2 \\
\hline $1-<2$ & 6.4 & 12 & 6.4 & 12 \\
\hline $2-<3$ & 6.4 & 12 & 6.4 & 12 \\
\hline $3-<6$ & 6.3 & 12 & 6.3 & 12 \\
\hline $6-<11$ & 4.3 & 8.2 & 4.3 & 8.2 \\
\hline $11-<16$ & 2.5 & 5.1 & 2.5 & 5.1 \\
\hline $16-<21$ & 2.5 & 5.1 & 2.5 & 5.1 \\
\hline $20-<50$ & 2.2 & 4.7 & 2.2 & 4.7 \\
\hline$\geq 50$ & 1.7 & 3.5 & 1.7 & 3.5 \\
\hline
\end{tabular}

Source: US-EPA Analysis of CSFII, 1994-96 and 1998, based on USDA, 2000 and US-EPA, 2000, modified from US-EPA, 2009.

\subsubsection{Total Food Intake}

A summary of recommended values for total food intake, on an asconsumed basis from the Exposure Factors Handbook (US-EPA, 2009), is presented in Table 6.8. The recommended intake rates are based on data from the US-EPA (2007b) analysis of CSFII data.

The US-EPA recommended values presented in Table 6.8 are well validated as the general assessment factors (GAFs, described in Section 1.1) were used to judge the quality of the underlying data used to derive the recommendations. The US-EPA has assigned the following confidence ratings for the five GAFs: 1 . Soundness - high, 2 . applicability and utility - medium, 3. clarity and completeness - medium, 4. variability and uncertainty - medium, and 5. evaluation and review - medium; the overall rating was medium. (US-EPA, 2009).

Table 6.8: Recommended Values for Per Capita Total Food Intake, As Consumed - as Presented in the Exposure Factors Handbook

\begin{tabular}{lcr}
\hline Age Group & Mean (g/kg-day) & $\mathbf{9 5}^{\text {th }}$ Percentile $(\mathbf{g} / \mathbf{k g}$-day) \\
\hline Children & & \\
$0-1$ month & 20 & 61 \\
$1-<3$ months & 16 & 40 \\
$3-<6$ months & 28 & 65 \\
$6-<12$ months & 56 & 134 \\
$1-<2$ years & 90 & 161 \\
$2-<3$ years & 74 & 126 \\
$3-<6$ years & 61 & 102 \\
$6-<11$ years & 40 & 70 \\
$11-<16$ years & 24 & 45 \\
$16-<21$ years & 18 & 35 \\
Adults & & \\
$20-<40$ years & & 30 \\
$40-<70$ years & 16 & 26 \\
$\geq 70$ years & 14 & 27
\end{tabular}

Note: Total food intake was defined as intake of the sum of all foods in the following major food categories: dairy, meats, fish, eggs, grains, vegetables, fruits, and fats. Beverages, sugar, candy, and sweets, and nuts and nut products were not included because they could not be categorized into the major food groups. Also, human milk intake was not included.

Source: US EPA re-analysis of CSFII 1994-96, 98 data (Based on US EPA, 2007b), modified from USEPA, 2009 


\subsubsection{Human Breast Milk and Lipids}

According to the Exposure Factors Handbook (US-EPA, 2009), among infants born in 2004, 73.8\% were breastfed postpartum, $41.5 \%$ at 6 months, and $20.9 \%$ at 12 months.

Seven key studies were identified which gave rise to the recommended values for human milk and lipid intake as presented in Table 6.9. These key studies among nursing mothers in industrialized countries have shown that average intakes among infants ranged from approximately 500 to $800 \mathrm{ml} /$ day, with the highest intake reported for infants 3 to below 6 months old. It should be noted that the decrease in human milk intake with age is likely a result of complementary foods being introduced as the child grows and not necessarily a decrease in total energy intake. Recommendations were converted to $\mathrm{ml} /$ day using a density of human milk of $1.03 \mathrm{~g} / \mathrm{ml}$ rounded up to two significant figures (US-EPA, 2009).

The US-EPA recommended values presented in Table 6.9 are well validated as the general assessment factors (GAFs, described in Section 1.1) were used to judge the quality of the underlying data used to derive the recommendations. The US-EPA has assigned the following confidence ratings for the five GAFs: 1 . Soundness - medium, 2. applicability and utility - medium, 3. clarity and completeness - medium, 4. variability and uncertainty - low, and 5. evaluation and review - high; the overall rating was medium. (US-EPA, 2009).

Table 6.9: Recommended Values for Human Breast Milk and Lipid Intake Rates for Exclusively Breastfed Infant - as Presented in the Exposure Factors Handbook

\begin{tabular}{|c|c|c|c|c|c|c|c|c|}
\hline \multirow[t]{3}{*}{ Age (months) } & \multicolumn{4}{|c|}{ Human Milk Intake } & \multicolumn{4}{|c|}{ Lipid Intake $^{a}$} \\
\hline & \multicolumn{2}{|c|}{ Mean } & \multicolumn{2}{|c|}{$95^{\text {th }}$ Percentile ${ }^{\mathrm{b}}$} & \multicolumn{2}{|c|}{ Mean } & \multicolumn{2}{|c|}{$95^{\text {th }}$ Percentile ${ }^{b}$} \\
\hline & $\mathrm{ml} /$ day & $\mathrm{ml} / \mathrm{kg}$-day & $\mathrm{ml} /$ day & $\mathrm{ml} / \mathrm{kg}$-day & $\mathrm{ml} /$ day & $\mathrm{ml} / \mathrm{kg}$-day & $\mathrm{ml} /$ day & $\mathrm{ml} / \mathrm{kg}$-day \\
\hline $0-<1$ & 510 & 150 & 950 & 220 & 20 & 6.0 & 38 & 8.7 \\
\hline $1-<3$ & 690 & 140 & 980 & 190 & 27 & 5.0 & 40 & 8.0 \\
\hline $3-<6$ & 770 & 110 & 1,000 & 150 & 30 & 4.2 & 42 & 6.1 \\
\hline $6-<12$ & 620 & 83 & 1,000 & 130 & 25 & 3.3 & 42 & 5.2 \\
\hline
\end{tabular}

a) The recommended value for lipid content of human milk is 4.0 percent.

b) Upper percentile is reported as mean plus 2 standard deviations.

Source: Neville et al., 1988, Pao et al., 1980, Butte et al., 1984, Dewey and Lönnerdal, 1983, Butte et al., 2000, Dewey et al., 1991b and Arcus-Arth et al., 2005, modified from US-EPA, 2009.

Note: All references are cited in US-EPA, 2009

\section{$6.3 \mathrm{WHO}$}

The WHO standard values for food intake are those recommended by the ICRP (ICRP, 1974, cited in WHO/IPCS, 1994 and 1999). These values are presented in Table 6.10. 
Table 6.10: WHO's Standard Values on Dietary Intake

\begin{tabular}{ll}
\hline Food & Daily Intake in per Day $^{\text {a }}$ \\
\hline Cereals & 323 (flour and milled rice) \\
Starchy Roots & 225 (sweet potatoes, cassava and other) \\
Sugar & 72 (includes raw sugar, excludes syrups and honey) \\
Pulses and Nuts & 33 (includes cocoa beans) \\
Vegetables and Fruits & 325 (fresh equivalent) \\
Meat & 125 (includes offal, poultry and game in terms of carcass weight, excluding slaughter fats) \\
Eggs & 19 (fresh equivalent) \\
Fish & 23 (landed weight) \\
Milk & 360 (excludes butter; includes milk products as fresh milk equivalent) \\
Fats and Oils & 31 (pure fat content) \\
\hline
\end{tabular}

a) Based on average of estimates for seven geographical regions.

Source: Modified from WHO/IPCS, 1994.

\subsection{Conclusion and Recommendations}

The data presented in the US-EPA EFH (US-EPA, 2009) as well as the European data are comprehensive, whereas the WHO data are limited.

In the REACH TGD Chapter R8 (ECHA, 2008a), the recommended values are stated as "taken from Gold et al., 1984 and ICRP, 1975"; however, it is not clear whether the recommendations are taken from one of these references or both. The recommended food consumption rates from ECETOC (ECETOC, 2001) are based on European data published in 1992. The Danish survey on dietary habits (DTU-FOOD, 2010) has been performed in the period 2003-2008. The recommended standard breast milk intake rates from ECETOC (ECETOC, 2001) are taken from the 1997 version of the Exposure Factors Handbook (US-EPA, 1997).

The US-EPA recommended values for food intake are well validated as the general assessment factors (GAFs, described in Section 2.1) were used to judge the quality of the underlying data used to derive the recommendations. In general, the US-EPA overall ratings were medium to high.

The WHO standard values on dietary intake (Table 6.10) are those recommended by the ICRP (ICRP, 1974).

It should be noted that the references "ICRP, 1974" and "ICRP, 1975" are to the same ICRP report (ICRP Report No. 23).

The US-EPA recommendations as well as the Danish data on dietary habits are considered as being the most valid values for food consumption rates today as these recommendations are based on analyses of the most recent data. The Danish data are considered as being more representative for Europeans in general.

There might be some differences between US and European infants regarding breast milk intake. However, according to ECETOC (ECETOC, 2001), breast milk consumption values for Swedish infants were similar to those of US infants. Thus, the more recent US-EPA recommendations (US-EPA, 2009) are considered as being representative for European infants as well. 
In conclusion, the Danish data on dietary habits presented in Table 6.1 are considered as being the most valid values for food consumption rates and are therefore recommended as default exposure factors for assessments of the European population in the context of REACH. For breast milk intake, the US-EPA recommendations presented in Table 6.9 are considered as being the most valid values today and are therefore recommended as default exposure factors for assessments of European infants in the context of REACH.

The recommended values can also be used in the context of other chemical regulations such as those for e.g., biocides, pesticides etc. 


\section{Soil and Dust Ingestion and Inhalation}

The ingestion of soil is a potential source of human exposure to chemical substances. The exposure is usually expressed as an average amount of soil ingested per unit time (e.g., mg/day).

The potential for exposure to contaminants via soil is greater for small children because they are naturally curious and often examine new objects by putting them into their mouth; this mouthing behaviour is considered to be a normal phase of childhood development. In addition to ingestion of soil through the mouthing of objects or hands, true eating of soil (geophagy) is also a source of oral exposure to chemicals for children. Furthermore, children tend to play on the ground and on the floor, which also may lead to an increased exposure to chemicals in soil and dust particles when compared to adults. Soil ingestion among children may be uneven as most children only ingest relatively small amounts, while a few children may consume larger amounts. Deliberate ingestion of larger amounts of soil is termed "pica" (pica, which is the Latin word for the magpie, refers to "the persistent eating of nonnutritive substances"). Adults may also ingest soil and dust particles that adhere to food, cigarettes, or their hands thereby being exposed to contaminants in soil and dust particles.

There are several methodologies presented in the literature related to soil and dust ingestion (US-EPA, 2009). Three methodologies combine biomarker measurements with measurements of the biomarker substance's presence in environmental media. The "tracer element" method measures quantities of specific elements present in faeces, urine, food and medications, yard soil, house dust, and sometimes also community soil and dust, and combines this information using certain assumptions about the elements' behaviour in the gastrointestinal tract to produce estimates of soil and dust quantities ingested. The "bio-kinetic model comparison" methodology compares results from a bio-kinetic model of lead exposure and uptake that predict blood lead levels, with biomarker measurements of lead in blood. The "lead isotope ratio" methodology involves measurements of different lead isotopes in blood and/or urine, food, water, and house dust and compares the ratio of different lead isotopes to infer sources of lead exposure that may include dust or other environmental exposures. A fourth methodology, the "survey response" methodology, offers indirect evidence of soil/dust ingestion behaviours where responses to survey questions regarding soil and dust ingestion of adults, caregivers, and/or children are analysed. 


\subsection{Europe}

In the Exposure to chemicals via house dust (RIVM, 2008), values for ingestion and inhalation of house dust is provided. Table 7.1 presents the default values, which have been based on several studies. In section 11.2.6, Table 11.3, intake of dust from chalk is presented.

Table 7.1: Default Values for House Dust Ingestion and Inhalation

\begin{tabular}{lrrr}
\hline & $\begin{array}{r}\text { Ingestion of House Dust } \\
(\mathbf{m g} / \text { day) }\end{array}$ & $\begin{array}{r}\text { Inhalation of House Dust } \\
\text { (mg/day) }\end{array}$ & $\begin{array}{r}\text { Total Intake of House } \\
\text { Dust (mg/day) }\end{array}$ \\
\hline $\begin{array}{l}\text { Adult } \\
\text { Child }\end{array}$ & 50 & 0.8 & 50 \\
& 100 & 2.0 & 100
\end{tabular}

Source: RIVM, 2008.

The Danish Environmental Protection Agency (D-EPA) has in their Guidance from the Environmental Protection Agency No. 5 (D-EPA, 2006) recommended values for intakes of soil as presented in Table 7.2. The recommendations are based on the 1997 version of the EFH (US-EPA, 1997).

Table 7.2: Recommended Values for Intake of Soil - as Presented in Guidance from the Danish Environmental Protection Agency No. 5, 2006.

\begin{tabular}{lr}
\hline Route of Exposure & Default Value \\
\hline Oral, maximum single intake & $10 \mathrm{~g}$ \\
Oral, mean daily intake & $0.1 \mathrm{~g} /$ day \\
Oral, $95^{\text {th }}$ percentile & $0.2 \mathrm{~g} /$ day \\
Dermal contact, mean daily & $1 \mathrm{~g} /$ day \\
Dermal contact, maximum & $10 \mathrm{~g} /$ day \\
\hline
\end{tabular}

Source: D-EPA, 2006.

In the Exposure Factors Sourcebook for European Populations, with Focus on UK Data (ECETOC, 2001), median soil ingestion rates have been estimated as $40 \mathrm{mg} /$ day and $1 \mathrm{mg} /$ day for children and adults, respectively. The data were based upon US studies ( Calabrese et al., 1997 and Stanek et al., 1997), but represented the best available information according to ECETOC (2001). Upper limits for soil ingestion of $200 \mathrm{mg} /$ day for children and $300 \mathrm{mg} /$ day for adults were considered to be supported by the available data. ECETOC has noted that the higher upper limit for adults is related to data variability and indicates the uncertainty in current estimates. It was also noted that the recommended values were based upon studies performed in the summer or autumn and that soil ingestion during winter is likely lower. 


\subsection{US-EPA}

In the Exposure Factors Handbook (EFH) (US-EPA, 2009), nine "key studies" and 28 "relevant studies" were identified. The key studies were used to recommend values for soil and dust ingestion for adults and children. The US-EPA has noted that studies estimating adult soil ingestion were extremely limited, and only two of these were considered to be key studies. Furthermore, there were no available studies estimating the ingestion of dust by adults and therefore, no recommended values were provided for adults for either dust or soil + dust ingestion. The USEPA has also noted that the key studies pre-dated the recommendations on age groups in the US-EPA's Guidance on Selecting Age Groups for Monitoring and Assessing Childhood Exposures to Environmental Contaminants (US-EPA 2005), and were performed on groups of children of varying ages.

Table 7.3 presents the central tendency recommendations for daily ingestion of soil, dust, or soil + dust, in $\mathrm{mg} /$ day and rounded to one significant figure. The soil ingestion recommendations represent ingestion of a combination of soil and outdoor settled dust, without distinguishing between these two sources. The dust ingestion recommendations include soil tracked into the indoor setting, indoor settled dust and airsuspended particulate matter that is inhaled and swallowed. The soil + dust recommendations are the sum of the soil and dust recommendations. The recommended values represent mass of ingested soil or dust on a dry weight basis. A summary of the nine key studies is presented in Table F.1 in Annex F.

Table 7.3 also presents the soil-pica or geophagy recommendations for daily ingestion of soil, in $\mathrm{mg} /$ day. No data were available on which to base comparable upper percentile recommendations for dust or soil + dust for adults or children. The US-EPA has noted that, due to the current state of research on soil and dust ingestion, the upper percentile recommendations are called "soil-pica" (the recurrent ingestion of unusually high amounts of soil, i.e., on the order of 1000-5000 mg/day or more) or "geophagy" (the intentional ingestion of earths, usually associated with cultural practises) recommendations that are likely to represent high soil ingestion episodes or behaviours at an unknown point on the high end of the distribution of soil ingestion. 
Table 7.3: Recommendation for Soil and Dust Ingestion - as Presented in the Exposure Factors Handbook

\begin{tabular}{|c|c|c|c|c|c|}
\hline \multirow[t]{3}{*}{ Age } & \multicolumn{3}{|l|}{ Soil $^{a}$} & Dust $^{b}$ & Soil and Dust \\
\hline & \multirow[b]{2}{*}{$\begin{array}{r}\text { Central Tendency } \\
\mathrm{mg} / \mathrm{day}\end{array}$} & \multicolumn{2}{|c|}{ Upper Percentile } & \multirow[b]{2}{*}{$\begin{array}{r}\text { Central Tendency } \\
\mathrm{mg} / \mathrm{day}\end{array}$} & \multirow[b]{2}{*}{$\begin{array}{r}\text { Central Tendency } \\
\mathrm{mg} / \text { day }\end{array}$} \\
\hline & & $\begin{array}{l}\text { Soil-Pica } \\
\mathrm{mg} / \text { day }\end{array}$ & $\begin{array}{r}\text { Geophagy } \\
\mathrm{mg} / \text { day }\end{array}$ & & \\
\hline $6<12$ months & 30 & - & - & 30 & 60 \\
\hline $1-<6$ years & 50 & 1,000 & 50,000 & 60 & $100^{c}$ \\
\hline $6-21$ years & 50 & 1,000 & 50,000 & 60 & $100^{c}$ \\
\hline Adult & 50 & - & 50,000 & - & - \\
\hline
\end{tabular}

-) No recommendation.

a) Includes soil and outdoor settled dust.

b) Includes indoor settled dust only.

c) Total soil and dust ingestion rate is $110 \mathrm{mg} /$ day; rounded to one significant figure it is $100 \mathrm{mg} / \mathrm{day}$.

Source: Modified from US-EPA, 2009.

The US-EPA recommended values for intake of soil and presented in Table 7.3 are well validated as the general assessment factors (GAFs, described in Section 2.1) were used to judge the quality of the underlying data used to derive the recommendations. The US-EPA has assigned the following confidence ratings for the five GAFs: 1 . Soundness - low, 2 . applicability and utility - low, 3. clarity and completeness - low, 4. variability and uncertainty - low, and 5. evaluation and review - medium; the overall rating was low. (US-EPA, 2009).

\subsection{WHO}

WHO (WHO, 1994) has specified the daily amount of soil ingested as 20 $\mathrm{mg} /$ day. This is a median value from the national Department of Health of the Government of Canada (Health and Welfare Canada, 1992). It has not been specified whether this standard value is valid for adults or for children.

\subsection{Conclusion and Recommendations}

The data presented in the US-EPA EFH (US-EPA, 2009) are the most comprehensive. There are some European data, but the WHO data are limited.

The Danish Environmental Protection Agency (D-EPA, 2006) has based their recommended values on the 1997 version of the EFH (USEPA, 1997).

The recommendations from ECETOC (ECETOC, 2001) are based upon two US studies. The recommendation for children is based on a study on children residing on a grassy Superfund site (Calabrese et al., 1997), included in the 2009 version of the EFH (US-EPA, 2009) as one of the nine key studies. According to ECETOC, it is possible that the physical 
nature of the site and possible changes in activity patterns associated with Superfund designation could have led to a depression in soil ingestion rates in this study. The recommendation for adults is based on a pilot study (Stanek et al., 1997); this study is not included in the 2009 version of the EFH (US-EPA, 2009).

The US-EPA recommended values for intake of soil (presented in Table 7.3) are well validated as the general assessment factors (GAFs, described in Section 2.1) were used to judge the quality of the underlying data used to derive the recommendations. The US-EPA overall rating was low. (US-EPA, 2009).

The WHO standard value on soil ingestion is a median value from the national Department of Health of the Government of Canada (Health and Welfare Canada, 1992). It has not been specified whether this standard value is valid for adults or for children.

Data on soil and dust ingestion rates are generally limited and variable, and region specific differences in child play and hygienic patterns will likely affect typical soil and dust ingestion rates. Thus, there might be some differences between the US and European population regarding soil and dust ingestion. Even though the US-EPA recommendations are considered as being the most valid values for soil and dust ingestion rates, the recommended values for house dust ingestion and inhalation rates for adults and children from RIVM (RIVM 2008) are considered as being representative for the European population. For children's dust ingestion the ECHA Guidance on information recuiremments and chemical safety assessment, Chapter R.15: Consumer exposure estimation (ECHA 2008b) also refer to the Dutch data. In conclusion, the RIVM recommended values wich are also referred in ECHA's REACH guidance R15, are presented in Table 7.1 and are considered as being the most valid values for dust ingestion rates in Europe as today and are therefore recommended as default exposure factors for assessments of the European population in the context of REACH. 


\section{Non-Dietary Ingestion Factors}

Adults and children have the potential for exposure to toxic substances through non-dietary ingestion pathways other than soil and dust ingestion, e.g., ingesting pesticide residues that have been transferred from treated surfaces to the hands or objects that are mouthed. Adult's mouth objects such as cigarettes, pens/pencils, or their hands. Young children mouth objects, surfaces or their fingers as they explore their environment. Mouthing behaviour includes all activities in which objects, including fingers, are touched by the mouth or put into the mouth except for eating and drinking, and includes licking, sucking, chewing, and biting (US-EPA, 2009).

\subsection{Europe}

In the Children's Toys Fact Sheet (RIVM, 2002), default values of hand-tomouth contact and direct ingestion are presented. As these values are based on various sorts of toys, they are described in the section on Consumer Products (Section 11.2.6).

In the Preparation of a Guidance Document on Pesticide Exposure; Assessment for Workers, Operators, Bystanders and Residents (EFSA, 2010), the default value for hand-to-mouth activity for children is set to 9.5 events per hour with a surface area of $20 \mathrm{~cm}^{2}$ of the mouthed hands.

\subsection{US-EPA}

In the Exposure Factors Handbook (EFH) (US-EPA, 2009) nine "key studies" and four "relevant studies" were identified regarding mouthing frequency, and three "key studies" and five "relevant studies" were identified regarding mouthing duration. The key studies were used to develop recommended values for mouthing frequency and duration, respectively, among children. No studies were located that provided data on mouthing frequency or duration for adults. US-EPA has noted that, in several cases, the key studies predated the recommendations on age groups in the US-EPA's Guidance on Selecting Age Groups for Monitoring and Assessing Childhood Exposures to Environmental Contaminants (US-EPA 2005), and were performed on groups of children of varying ages. In the cases in which age groups of children in the key studies did not correspond exactly to the US-EPA's recommended age groups, the closest age group was used.

Table 8.1 presents the recommended mouthing frequencies, expressed in units of contacts per hour, between either an object or surface and the 
mouth (object-to-mouth), or between any part of the hand (including fingers and thumbs) and the mouth (hand-to-mouth). The recommended hand-to-mouth frequencies are based on data from a recent study (Xue et al., 2007, cited in US-EPA, 2009) in which a secondary analysis of data from several of the studies summarized in the EFH, as well as data from unpublished studies, was conducted. The recommended object-to-mouth frequencies are based on data from a very recent study (Xue et al., 2009, cited in US-EPA, 2009) in which a secondary analysis of data from several of the studies summarized in the EFH was conducted. Recommendations for duration of object-to-mouth contacts are based on data from the three key studies. Recommendations for hand-to-mouth duration are not provided in the EFH since it was considered that those estimates may not be relevant to environmental exposures. (US-EPA, 2009).

Table 8.1: Recommendations on Mouthing Frequency and Duration among Children - as Presented in the Exposure Factors Handbook

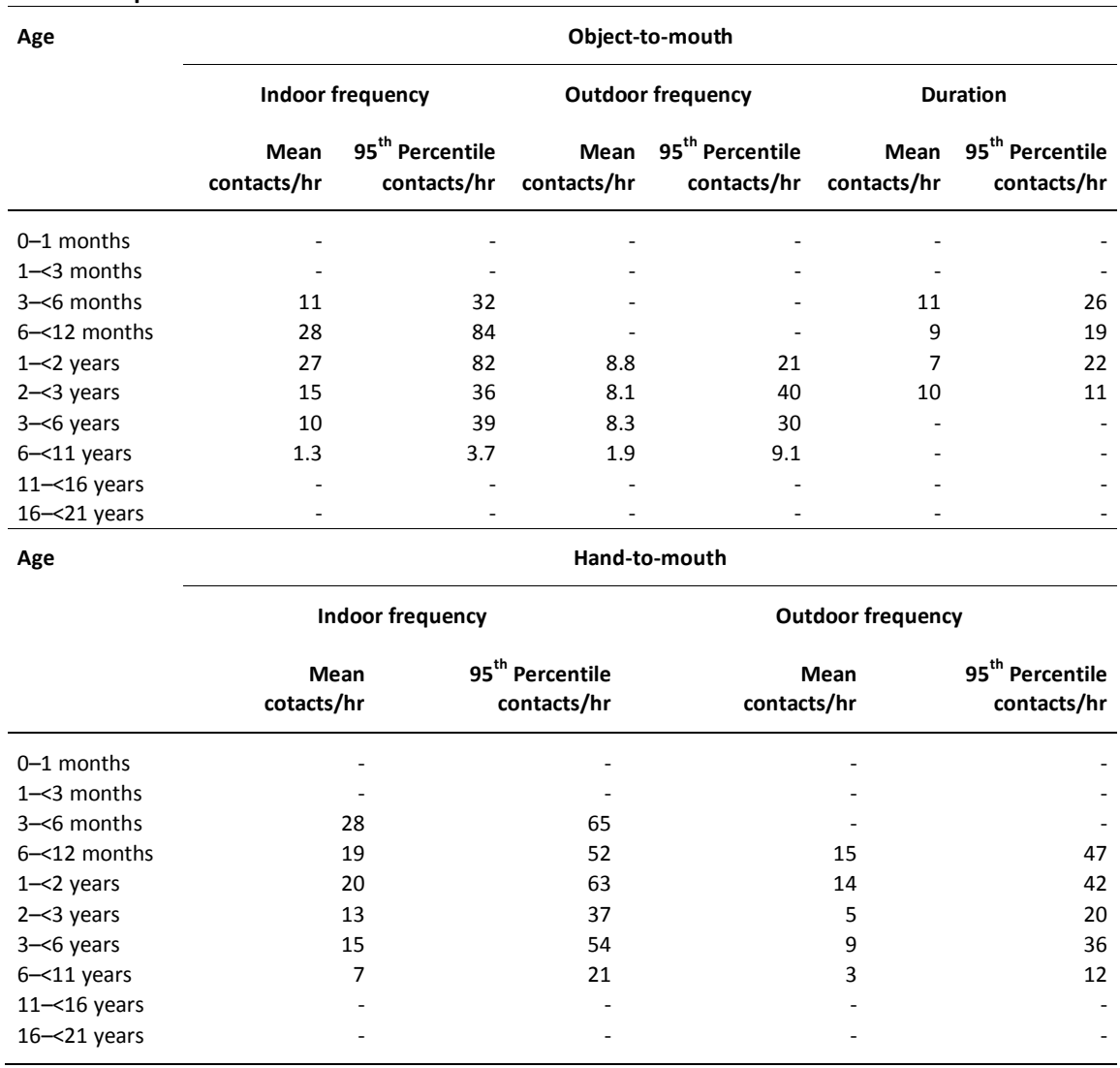

Sources: "Hand-to-mouth" frequencies: Xue et al., 2007. “Object-to-mouth" frequencies: Xue et al. 2009. "Mouthing duration": Juberg et al., 2001; Greene et al., 2002 and Beamer et al., 2008, modified from US-EPA, 2009.

Note: All references are cited in US-EPA, 2009.

The US-EPA recommended values for non-dietary ingestion factors presented in Table 8.1 are well validated as the general assessment factors (GAFs, described in Section 2.1) were used to judge the quality of the underlying data used to derive the recommendations. The US-EPA has 
assigned the following confidence ratings for the five GAFs: 1 . Soundness - low, 2. applicability and utility - low, 3. clarity and completeness - low, 4. variability and uncertainty - low, and 5. evaluation and review - medium; the overall rating was low for both frequency and duration of hand-to-mouth and object-to-mouth. (US-EPA, 2009).

\subsection{WHO}

No recommendations on non-dietary ingestion factors are provided by the WHO.

\subsection{Conclusion and Recommendations}

The data presented in the US-EPA EFH (US-EPA, 2009) are the most comprehensive. The European data are limited to single values for specific assessment purposes. No recommendations have been provided by the WHO.

There are no references provided for the recommended value for hand-to-mouth activity for children from EFSA (EFSA, 2008).

The US-EPA recommended values for non-dietary ingestion factors presented in Table 8.1 are well validated as the general assessment factors (GAFs, described in Section 2.1) were used to judge the quality of the underlying data used to derive the recommendations. The US-EPA overall rating was low for both frequency and duration of hand-tomouth and object-to-mouth. (US-EPA, 2009).

There might be some differences between US and European children regarding hand-to-mouth and hand-to-object frequency and duration. Despite this, the US-EPA recommended values are considered as being representative for European children as well. Furthermore, the US-EPA recommendations are considered as being the most valid values for nondietary ingestion factors as today despite the overall rating as low, as these recommendations are based on relatively recent key studies published in the period 2001-2008.

In conclusion, the US-EPA recommended values presented in Table 8.1 are considered as being the most valid values for non-dietary ingestion factors as today and are therefore recommended as default exposure factors for assessments of European children in the context of REACH. No studies have been located on mouthing frequency or duration for adults and therefore, no default exposure factors can be recommended. 


\section{Lifetime Expectancy}

The length of an individual's life is an important factor to consider when evaluating cancer risk because the dose estimate is averaged over an individual's lifetime. Since the averaging time is found in the denominator of the dose equation, a shorter lifetime would result in a higher potential risk estimate, and conversely, a longer life expectancy would produce a lower potential risk estimate. (US-EPA, 2009).

\subsection{Europe}

In the REACH Guidance on information requirements and chemical safety assessment Chapter R8 (ECHA, 2008a), the recommended lifespan is 75 years for exposure assessments of consumers and man exposed indirectly via the environment.

According to the Exposure Factors Sourcebook for European Populations, with Focus on UK Data (ECETOC, 2001), the average life expectancy of a UK child born in 1998 is 78 years. Life expectancies for European countries are presented in Annex G, Table G.1 and recent statistics on life expectancy for England are provided presented by sex in Table G.2.

\subsection{US-EPA}

According to the Exposure Factors Handbook (US-EPA, 2009), current data suggest that 78 year is an appropriate value to reflect the average life expectancy of the general population and is therefore the recommended value. It should be noted, that if gender is a factor considered in the assessment, the average life expectancy value for females is higher than for males. It is recommended that the assessor use the appropriate value of 75 years for males or 80 years for females, based on life expectancy data from 2005 (US National Centre for Health Statistics, 2008). Table 9.1 presents recommended values for life expectancy at birth.

The US-EPA recommended values for lifetime expectancy are well validated as the general assessment factors (GAFs, described in Section 2.1) were used to judge the quality of the underlying data used to derive the recommendations. The US-EPA has assigned the following confidence ratings for the five GAFs: 1 . Soundness - medium to high, 2 . applicability and utility - high, 3. clarity and completeness - high, 4. variability and uncertainty - medium, and 5. evaluation and review - high. The overall rating was high. 
Table 9.1: Recommended Values for Expectation of Life at Birth: 2005 - as Presented in the Exposure Factors Handbook

\begin{tabular}{lr}
\hline Population & Life Expectancy (years) \\
\hline Men & 75 \\
Women & 80 \\
Total & 78 \\
\hline
\end{tabular}

Source: US National Centre for Health Statistics, 2008; modified from US-EPA, 2009

\subsection{WHO}

WHO has estimated worldwide life expectancy at birth for the year 2008 (WHO, 2010). Table 9.2 presents life expectancy at birth for all European countries combined and global. In Annex F, Table F.3., life expectancy at birth for all European countries in 2008, is presented.

Table 9.2: Life Expectancy at Birth for the European Countries and Global, WHO 2008

\begin{tabular}{lr}
\hline Population & Life Expectancy (years) \\
\hline European Region & 71 \\
Men & 79 \\
Women & 75 \\
Men and women & 66 \\
Global & 70 \\
Men & 68 \\
Women & \\
Men and women & \\
\hline
\end{tabular}

Source: WHO, 2010.

\subsection{Conclusion and Recommendations}

In the REACH TGD Chapter R8 (ECHA, 2008a), the recommended values are stated as "taken from Gold et al., 1984 and ICRP, 1975"; however, it is not clear whether the recommendations are taken from one of these references or both. The recommendation from ECETOC is based on a WHO report from 1999.

The US-EPA recommended values for lifetime expectancy are well validated as the general assessment factors (GAFs, described in Section 2.1) were used to judge the quality of the underlying data used to derive the recommendations. The US-EPA overall rating was high.

The WHO standard values are from very recent statistics published in 2010.

Life expectancy is very different for various countries in the World. The very recent data published by the WHO in 2010 are considered as being the most representative for European lifetime expectancy as today. 
In conclusion, the WHO recommended values for the European Region presented in Table 9.2 are considered as being the most valid values for lifetime expectancy as today and are therefore recommended as default exposure factors for assessments of the European population in the context of REACH. 


\section{Activity Factors}

Individual or group activities are important determinants of potential exposure, because toxic chemicals introduced into the environment may not cause harm to an individual until an activity is performed that subjects the individual to contact with those contaminants. An activity or time spent in a given activity will vary among individuals on the basis of, for example, culture, ethnicity, hobbies, location, gender, age, socioeconomic characteristics, and personal preferences. (US-EPA, 2009).

\subsection{Europe}

In the REACH Guidance on information requirements and chemical safety assessment Chapter R8 (ECHA, 2008a), the recommended length of a workday is set to 8 hours, working days per week are 5 , working weeks per year are recommended to 48 weeks, and worklife is 40 years.

Table 10.1. presents recommended values for activity factors for UK adults and children according to the Exposure Factors Sourcebook for European Populations, with Focus on UK Data (ECETOC, 2001).

\begin{tabular}{|c|c|}
\hline Activity Scenario & Mean/Median Time Spent \\
\hline Work Hours per Week & $38^{\mathrm{a}}$ hours/day \\
\hline Indoors (Adults) & 22 hours/day \\
\hline Outdoors (Adults) & 2 hours/day \\
\hline School Day for Children & $7^{\mathrm{b}}$ hours/day (for 190 day per year) \\
\hline Indoors & 5 hours/day \\
\hline Outdoors & 2 hours/day \\
\hline \multicolumn{2}{|l|}{ Time Spent at Home } \\
\hline For Full-Time Workers & 15 hours/day (14 indoors, 1 outdoors) \\
\hline For individuals not employed outside of the home & 20.5 hours/day (18.5 indoors, 2 outdoors) \\
\hline \multicolumn{2}{|l|}{ Time Spent Away } \\
\hline For Full-Time Workers & 9 hours/day \\
\hline For individuals not employed outside of the home & 3.5 hours/day \\
\hline Outdoor Recreation (for Adults) & 0.3 hours/day (for 365 day per year) \\
\hline Employment Tenure $^{\mathrm{c}}$ & 8 years \\
\hline Residential Tenure & 9 years \\
\hline \multirow[t]{2}{*}{ School Tenure } & 7 years primary school \\
\hline & 5 years secondary school \\
\hline
\end{tabular}

a) Based upon the assumption of 6 weeks for combined vacation and leave, an estimate of 230 work days/year (46 weeks at 5 days/week) is obtained.

b) For secondary school children involved in extracurricular activities, a value of 8 hours/day may be more representative.

c) Time spent working for current employer.

Source: Modified from ECETOC, 2001. 


\subsection{US-EPA}

In the Exposure Factors Handbook (US-EPA, 2009), recommended standard values for activity factors are based on different key studies and values for various scenarios are presented in Table 10.2.

The values presented in Table 10.2 are well validated as the general assessment factors (GAFs, described in Section 2.1) were used to judge the quality of the underlying data used to derive the recommendations. The US-EPA has assigned the following confidence ratings for the five GAFs: 1 . Soundness -high, 2 applicability and utility - medium, 3. clarity and completeness - medium, 4. variability and uncertainty - medium, and 5. evaluation and review - medium. The overall rating was medium for the mean and low for upper percentile.

\begin{tabular}{|c|c|c|c|}
\hline Age group & Mean & $95^{\text {th }}$ Percentile & Reference \\
\hline \multicolumn{4}{|c|}{ Time Indoors (Total) in minutes per day } \\
\hline 0-1 month & 1,440 & - & \\
\hline $1-<3$ months & 1,432 & - & \\
\hline $3-<6$ months & 1,414 & - & US-EPA analysis of source data from Wiley et \\
\hline $6-<12$ months & 1,301 & - & al., 1991 for age groups from birth to $<12$ \\
\hline $1-<2$ years & 1,353 & - & months. \\
\hline $2-<3$ years & 1,316 & - & \\
\hline $3-<6$ years & 1,278 & - & \\
\hline $6-<11$ years & 1,244 & - & US-EPA re-analysis of source data from US-EPA, \\
\hline $11-<16$ years & 1,260 & - & 1996 for age groups from 1 to $<21$ years. \\
\hline $16-<21$ years & 1,248 & - & \\
\hline $18-<65$ years & 1,159 & - & \\
\hline$\geq 65$ years & 1,142 & - & Adults, $\geq 18$ years: US-EPA, 1996 \\
\hline \multicolumn{4}{|c|}{ Time Outdoors (Total) in minutes per day } \\
\hline $0-1$ month & 0 & - & \\
\hline $1-<3$ months & 8 & - & \\
\hline $3-<6$ months & 26 & - & Children, Birth to $<12$ months: US-EPA analysis \\
\hline $6-<12$ months & 139 & - & of source data from Wiley et al., 1991. \\
\hline $1-<2$ years & 36 & - & \\
\hline $2-<3$ years & 76 & - & \\
\hline $3-<6$ years & 107 & - & Children, 1 to $<21$ years: US-EPA re-analysis of \\
\hline $6-<11$ years & 132 & - & source data from US-EPA, 1996. \\
\hline $11-<16$ years & 100 & - & \\
\hline $16-<21$ years & 102 & - & \\
\hline $18-<65$ years & 281 & - & Adults, $\geq 18$ years: US-EPA, 1996. \\
\hline$\geq 65$ years & 298 & - & \\
\hline \multicolumn{4}{|c|}{ Time Indoors (at Residence) in minutes per day } \\
\hline $0-<1$ year & 1,108 & 1,440 & \\
\hline $1-<2$ years & 1,065 & 1,440 & \\
\hline $2-<3$ years & 979 & 1,296 & Children, Birth to $<21$ years: US-EPA re-analysis \\
\hline $3-<6$ years & 957 & 1,355 & of source data from US-EPA, 1996. \\
\hline $6-<11$ years & 893 & 1,275 & \\
\hline $11-<16$ years & 889 & 1,315 & \\
\hline $16-<21$ years & 833 & 1,288 & Adults $\geq 18$ years: US-EPA, 1996 . \\
\hline $18-<65$ years & 948 & 1,428 & \\
\hline$\geq 65$ years & 1,175 & 1,440 & \\
\hline \multicolumn{4}{|c|}{ Showering in minutes per day } \\
\hline $0-<1$ year & 15 & - & \\
\hline $1-<2$ years & 20 & - & \\
\hline $2-<3$ years & 22 & 44 & US-EPA re-analysis of source data from \\
\hline $3-<6$ years & 17 & 34 & US-EPA, 1996. \\
\hline $6-<11$ years & 18 & 41 & \\
\hline $11-<16$ years & 18 & 40 & \\
\hline $16-<21$ years & 20 & 45 & \\
\hline
\end{tabular}




\begin{tabular}{|c|c|c|c|}
\hline Age group & Mean & $95^{\text {th }}$ Percentile & Reference \\
\hline \multicolumn{4}{|c|}{ Bathing in minutes per day } \\
\hline $0-<1$ year & 19 & 30 & \\
\hline $1-<2$ years & 23 & 32 & \\
\hline $2-<3$ years & 23 & 45 & US-EPA re-analysis of source data from \\
\hline $3-<6$ years & 24 & 60 & US-EPA, 1996. \\
\hline $6-<11$ years & 24 & 46 & \\
\hline $11-<16$ years & 25 & 43 & \\
\hline $16-<21$ years & 33 & 60 & \\
\hline \multicolumn{4}{|c|}{ Bathing and Showering in minutes per day } \\
\hline $18-<65$ years & 17 & - & US-EPA, 1996. \\
\hline$\geq 65$ years & 17 & - & \\
\hline \multicolumn{4}{|c|}{ Swimming in minutes per month } \\
\hline $0-<1$ year & 96 & - & \\
\hline $1-<2$ years & 105 & - & \\
\hline $2-3$ years & 116 & 181 & Children, Birth to $<21$ years: US EPA re-analysis \\
\hline $3-<6$ years & 137 & 181 & of source data from US EPA, 1996. \\
\hline $6-<11$ years & 151 & 181 & \\
\hline $11-<16$ years & 139 & 181 & \\
\hline $16-<21$ years & 145 & 181 & Adults, $\geq 18$ years: US EPA, 1996. \\
\hline $18-<65$ years & $45^{\mathrm{a}}$ & 181 & \\
\hline$\geq 65$ years & $40^{\mathrm{a}}$ & 181 & \\
\hline \multicolumn{4}{|c|}{ Playing on Sand/Gravel in minutes per day } \\
\hline $0-<1$ year & 18 & - & \\
\hline $1-<2$ years & 43 & 121 & \\
\hline $2-<3$ years & 53 & 121 & Children, Birth to $<21$ years: US EPA re-analysis \\
\hline $3-<6$ years & 60 & 121 & of source data from US EPA, 1996. \\
\hline $6-<11$ years & 67 & 121 & \\
\hline $11-<16$ years & 67 & 121 & \\
\hline $16-<21$ years & 83 & - & Adults, $\geq 18$ years: US EPA, 1996. \\
\hline $18-<65$ years & $0^{\mathrm{a}}$ & 121 & \\
\hline$\geq 65$ years & $0^{\mathrm{a}}$ & - & \\
\hline \multicolumn{4}{|c|}{ Playing on Grass in minutes per day } \\
\hline $0-<1$ year & 52 & - & \\
\hline $1-<2$ years & 68 & 121 & \\
\hline $2-<3$ years & 62 & 121 & Children, Birth to $<21$ years: US EPA re-analysis \\
\hline $3-<6$ years & 79 & 121 & of source data from US EPA, 1996. \\
\hline $6-<11$ years & 73 & 121 & \\
\hline $11-<16$ years & 75 & 121 & \\
\hline $16-<21$ years & 60 & - & Adults, $\geq 18$ years: US EPA, 1996. \\
\hline $18-<65$ years & $60^{\mathrm{a}}$ & 121 & \\
\hline$\geq 65$ years & $121^{\mathrm{a}}$ & - & \\
\hline \multicolumn{4}{|c|}{ Playing on Dirt in minutes per day } \\
\hline $0-<1$ year & 33 & - & \\
\hline $1-<2$ years & 56 & 121 & \\
\hline $2-<3$ years & 47 & 121 & Children, Birth to $<21$ years: US EPA re-analysis \\
\hline $3-<6$ years & 63 & 121 & of source data from US EPA, 1996. \\
\hline $6-<11$ years & 63 & 121 & \\
\hline $11-<16$ years & 49 & 121 & \\
\hline $16-<21$ years & 30 & - & Adults, $\geq 18$ years: US EPA, 1996. \\
\hline $18-<65$ years & $0^{\mathrm{a}}$ & 121 & \\
\hline$\geq 65$ years & $0^{\mathrm{a}}$ & - & \\
\hline
\end{tabular}

- Percentiles were not calculated for sample sizes less than 10 or in cases where the mean was calculated by summing the means from multiple locations or activities.

a) Median value, mean not available in US-EPA, 1996.

Note: All activities are reported in units of minutes/day, except swimming, which is reported in units of minutes/month.

There are 1,440 minutes in a day. Time indoors and outdoors may not add up to 1,440 minutes due to activities that could not be classified as either indoors or outdoors.

Source: Modified from US-EPA, 2009. 


\section{$10.3 \mathrm{WHO}$}

No recommendations on activity factors are provided by WHO.

\subsection{Conclusion and Recommendations}

The data presented in the US-EPA EFH (US-EPA, 2009) are the most comprehensive. The European data are limited. No recommendations have been provided by the WHO.

In the REACH TGD Chapter R8 (ECHA, 2008a), the recommendations are stated as "taken from Gold et al., 1984 and ICRP, 1975"; however, it is not clear whether the recommendations are taken from one of these references or both. The recommended values from ECETOC are based on different sources published in the 1990'ies.

The US-EPA recommended values are well validated as the general assessment factors (GAFs, described in Section 2.1) were used to judge the quality of the underlying data used to derive the recommendations. The USEPA overall rating was medium for the mean and low for upper percentile.

Activity levels might be different among US individuals compared to Europeans. Despite this, the US-EPA recommended values are considered as being representative for Europeans as well. Furthermore, the US-EPA recommendations are considered as being the most valid values for activity factors. It should be noted that the recommended values in the 2009 version of the EFH (US-EPA, 2009) are based on two key studies published in 1991 and 1996 and the ECETOC recommendations are based on different sources published in the 1990'ies.

In conclusion, the US-EPA recommended values presented in Table 10.2 are considered as being the most valid values for activity factors for Europeans as today and are therefore recommended as default exposure factors for assessments of European inhabitants in the context of REACH. No default values for working activities are recommended by the US-EPA as the working environment is not the responsibility of USEPA. For working activities, the recommended values presented in Table 10.1 are recommended as default exposure factors for assessments of European workers in the context of REACH. 


\section{Consumer Products}

Consumer products may contain toxic or potentially toxic chemical constituents to which people may be exposed as a result of their use. For example, household cleaners can contain ammonia, alcohols, acids, and/or organic solvents which may pose health concerns. Potential routes of exposure to consumer products or chemicals released from consumer products during use include ingestion, inhalation, and dermal contact. These household consumer products include cleaners, solvents, and paints. Non-users, including children, can be passively exposed to chemicals in these products. Since people spend a large amount of time indoors, the use of household chemicals in the indoor environment can be a principal source of exposure. (US-EPA, 2009).

In order to estimate consumer product exposure, three exposure routes are to be considered: inhalation, dermal and oral routes, each exposure calculated separately (RIVM, 2006a):

\section{Inhalation}

It is assumed that all substances are released as a gas, vapour or airborne particulate into a standard room. This may be due to direct release or to evaporation from a liquid or a solid matrix

\section{Dermal}

Two options:

- The substance is contained in a preparation. This option is applicable when, for example, hands are put into a solution containing the substance under evaluation, or splashes occur (painting)

- Substance migrating from an article; applicable, for example, when residual dyes in clothing are in contact with skin and migrate from the clothing

\section{Oral}

Two options:

- Substance in a product unintentionally swallowed during normal use

- Substance migrating from an article; applicable for example when a substance migrates from a pen, cutlery or textile 


\subsection{Europe}

As mentioned in section 1.1.2, RIVM has provided Fact Sheets with default values on several consumer products which are calculated using the ConsExpo software model. The default values in the fact sheets have been collected for Dutch consumers (non-professional users). They are not aimed at describing exposure for people who professionally work with consumer products, such as hairdressers and in hospitals, for example. These fact sheets, therefore, only describes consumer products which are available to the consumer for private use. The parameter values in the fact sheets are chosen such that a relatively high exposure and uptake are calculated, in the order of magnitude of a $99^{\text {th }}$ percentile of the distribution. To achieve this goal, the $75^{\text {th }}$ or the $25^{\text {th }}$ percentile is calculated (or estimated) for each parameter. The $75^{\text {th }}$ percentile is used for parameters which give a higher exposure for higher values, and the $25^{\text {th }}$ percentile is used in the reverse case (RIVM, 2006a).In order to assess the exposed area, RIVM uses the default value for surface areas from the General Fact Sheets (RIVM, 2006a) (see Section 3.1) and the Exposure Factors Handbook (US-EPA, 2009) (see Section 3.2).

\subsubsection{Cosmetics}

The Cosmetics Fact Sheet (RIVM, 2006b) covers the use of cosmetics by consumers for 35 product categories including shampoo, make-up, lipstick, deodorant and toothpaste. Default models and values for all 35 product categories have been determined to assess exposure and intake of compounds in cosmetics (RIVM, 2006b).

Making exposure assessment on sprays can be very complicated and several parameters must be taken in to account - the room size where the spaying process takes place, the size of the droplets and the composition of the substance. Especially the size is important. During spraying the user can inhale droplets of the product. Sprays produce an aerosol cloud of very small to small droplets. The speed with which the droplets fall depends on the size of the droplet; smaller droplets stay in the air longer and smaller droplets are more prone to be inhaled. In the Cosmetics Fact Sheet (RIVM, 2006b), different terminologies are used in order to describe the exposure:

- Cloud volume: During the actual spraying of a cosmetic product towards a person, the person is exposed to an aerosol cloud with fine particles. In the ConsExpo 4.0 spray model, the volume of the cloud after 1 second is assumed to further increase linearly in time until spraying stops or the cloud volume equals the volume of the room. It is assumed that during the use of the spray (the actual spraying) the breathing zone of the exposed person is located inside this volume. After spraying, the sprayed material is assumed to be homogeneously 
dispersed over the entire room. The default value for cloud volume is set at $1 / 16 \mathrm{~m}^{3}$ or $0.0625 \mathrm{~m}^{3}$. This cloud volume matches a cone measuring $1 \mathrm{~m}$ (length) and $0.5 \mathrm{~m}$ (diameter); in addition, it matches a sphere with a diameter of $0.5 \mathrm{~m}$

- Airborne fraction: The airborne fraction is the fraction of non-volatile material that becomes airborne in the form of droplets. The airborne fraction depends on the way it is used, i.e. spraying on a surface (paint, wood preservative) or spraying in the air (spraying against flies), and on the droplet size distribution that has been specified. Several airborne fractions has been investigated and based on these measures, the default value for air space sprays and surface sprays with a median of the initial particle distribution $<50 \mu \mathrm{m}$ is 1 and the default value for surface sprays with a median of the initial particle distribution $\geq 50 \mu \mathrm{m}$ is 0.2

- Initial particle distribution: As mentioned earlier, the droplet size is an important parameter when estimating the exposure. Smaller drops fall at a lower speed and stay in the air for longer. The large droplets will quickly disappear from the air after being formed. As an indication: the falling time of droplets with a diameter of $100 \mu \mathrm{m}$ from a height of 3 meters is calculated at $11 \mathrm{sec}$, and for droplets of $10 \mu \mathrm{m}$ it is calculated at $17 \mathrm{~min}$

- Inhalation cut-off diameter: The inhalation cut-off diameter is the measure for the diameter of the spray droplets that can be inhaled and reach the lower areas of the lungs (alveoli, bronchioles, bronchia). Particles that are above this diameter deposit in the higher parts of the respiratory tract and will be cleared via the gastro-intestinal tract, leading to oral exposure. The inhalation cut-off diameter is only an approximation of the complicated process of deposition of particles in the lung. In general its value should be around 10-15 micrometer. According to the RIVM, the default value is set at $15 \mu \mathrm{m}$

- Density: Many non-volatile substances in cosmetics are made of large organic compounds with densities usually between 1.0 and 1.5 $\mathrm{g} / \mathrm{cm}^{3}$. For a complex mixture of (especially organic) compounds, the density is set at $1.8 \mathrm{~g} / \mathrm{cm}^{3}$. The density of salts generally varies between 1.5 and $3.0 \mathrm{~g} / \mathrm{cm}^{3}$ (RIVM, 2006b)

In Table 11.1 a summary of general default values for cosmetic sprays is presented and in Table 11.2, default values for the density for solvents and for non-volatile compounds are presented. 
Table 11.1: General Default Values for Cosmetic Sprays

\begin{tabular}{ll}
\hline Inhalation, spray model: spraying towards exposed person & Default value \\
\hline Cloud volume & \\
Spray cans & $0.0625 \mathrm{~m}^{3}$ \\
Pump sprays & $0.0625 \mathrm{~m}^{3}$ \\
Airborne fraction & \\
Airspace sprays/surface sprays; median of the initial particle distribution $<50 \mu \mathrm{m}$ & $1 \mathrm{~g} / \mathrm{g}$ \\
Surface sprays; median of the initial particle distribution $\geq 50 \mu \mathrm{m}$ & $0.2 \mathrm{~g} / \mathrm{g}$ \\
Inhalation cut-off diameter & $15 \mu \mathrm{m}$ \\
\hline
\end{tabular}

Source: Modified from the Cosmetics Fact Sheet, RIVM (2006b)

Table 11.2: Default values for density

\begin{tabular}{llr}
\hline Type & Main Ingredient & Density $\left[\mathrm{g} / \mathrm{cm}^{3}\right]$ \\
\hline \multirow{2}{*}{ Solvents } & Volatile organic solvents & 0.7 \\
& Water & 1 \\
\multirow{2}{*}{ Non-volatile compounds } & Large organic compounds & 1.5 \\
& Salts & 3.0 \\
& Complex mixture of compounds, especially organic compounds & 1.8 \\
\hline
\end{tabular}

Source: Modified from the Cosmetics Fact Sheet, RIVM (2006b).

In the Cosmetics Fact Sheet (RIVM, 2006b) default values on the use of various cosmetic products are provided and selected default values are presented in Table 11.3. It should be noted that the majority of the products are assumed only to be used by women with a body weight of $61 \mathrm{~kg}$. The "normal" use of cosmetics by children, such as the use of baby salves, baby powder, sunscreen lotions and toothpaste, for example, is presented in Table 11.3. Other cosmetic products used by children are presented in Section 11.2.6, in Table 11.15. It is assumed that the room temperature is $20^{\circ} \mathrm{C}$, that the room height is $2.5 \mathrm{~m}$ and that the uptake fraction (inhalation and oral) is 1.

Table 11.3: Default values of Cosmetic Products

\begin{tabular}{|c|c|c|}
\hline & Default Value & Comments \\
\hline \multicolumn{3}{|l|}{ Shampoo } \\
\hline Frequency & 260 x/year & $\begin{array}{l}\text { Studies range between } 2-7 \text { times a week (default: } \\
260 \times / \text { year }=5 \times / \text { week) }\end{array}$ \\
\hline \multicolumn{3}{|l|}{ Dermal, instant application } \\
\hline Exposed area & $1440 \mathrm{~cm}^{2}$ & Area hands $+1 / 2$ area head \\
\hline Amount upon head dilution & $60 \mathrm{~g}$ & $\begin{array}{l}\text { The estimation is that wet hair contains } 40 \mathrm{~g} \text { water + } \\
\text { shampoo } 20 \mathrm{~g}\end{array}$ \\
\hline Weight fraction dilution & $W_{f} / 3$ & $\begin{array}{l}\text { Shampoo } 20 \mathrm{~g} \text {, total amount upon head } 60 \mathrm{~g} \text { : dilution } \\
\text { factor } 3\end{array}$ \\
\hline Exposure time & $4 \mathrm{~min}$ & $\begin{array}{l}\text { Mean: } 1 \text { min., max: } 5-10 \mathrm{~min} \text {,; assuming that sham- } \\
\text { poo after application is not rinsed out immediately }\end{array}$ \\
\hline \multicolumn{3}{|l|}{ Conditioner } \\
\hline Frequency & 104 x/year & $\begin{array}{l}\text { Studies range between } 1-2 \text { times a week (de- } \\
\text { fault:104/ year }=2 / \text { week) }\end{array}$ \\
\hline \multicolumn{3}{|l|}{ Dermal, instant application } \\
\hline Exposed area & $1440 \mathrm{~cm}^{2}$ & Area hands $+1 / 2$ area head \\
\hline Amount upon head dilution & $54 \mathrm{~g}$ & $\begin{array}{l}\text { The estimation is that wet hair contains } 40 \mathrm{~g} \text { water }+ \\
\text { conditioner } 14 \mathrm{~g}\end{array}$ \\
\hline Weight fraction dilution & $W_{f} / 3.9$ & $\begin{array}{l}\text { Conditioner } 14 \mathrm{~g} \text {, total amount upon head } 54 \mathrm{~g} \text { : } \\
\text { dilution factor } 3.9\end{array}$ \\
\hline Exposure time & $4 \mathrm{~min}$ & $\begin{array}{l}\text { Mean: } 1 \text { min., max: } 5-10 \mathrm{~min} \text {,; assuming that condi- } \\
\text { tioner after application is not rinsed out immediately }\end{array}$ \\
\hline
\end{tabular}




\begin{tabular}{|c|c|c|}
\hline & Default Value & Comments \\
\hline \multicolumn{3}{|l|}{ Hairspray, aerosol can ${ }^{\text {a) }}$} \\
\hline \multirow[t]{3}{*}{ Frequency } & $438 \times /$ year & Studies range between $1-2$ times per day; mean: 0.76 \\
\hline & & SD: 0.68 (default is calculated as follows: $75 \%$ mean: \\
\hline & & 0.76 a day and SD: 0.68 gives $1.2 \mathrm{x} /$ day i.e. $438 \mathrm{x} /$ year) \\
\hline \multicolumn{3}{|c|}{ Inhalation, spray model, spraying towards exposed person } \\
\hline \multirow[t]{3}{*}{ Spray duration } & $0.24 \mathrm{~min}$ & Studies range between $0.17-0.34$ min per day; mean: \\
\hline & & $0.18 \mathrm{SD}: 0.1 \mathrm{~min}$. (default is calculated as follows: \\
\hline & & $75 \%$ mean: 0.18 and SD: 0.1 min. gives 0.24 min.) \\
\hline Exposure duration & $5 \mathrm{~min}$ & Estimate: time in bathroom \\
\hline Room volume & $10 \mathrm{~m}^{3}$ & Bathroom \\
\hline Airborne fraction & 1 & see section 11.2 \\
\hline Weight fraction non-volatile & $0.03 \mathrm{~g} / \mathrm{g}$ & \\
\hline Density non-volatile & $1.5 \mathrm{~g} / \mathrm{cm}^{3}$ & see section 11.2 \\
\hline Initial particle distribution & $35 \mu \mathrm{m}(0.3)$ & see section 11.2 \\
\hline \multicolumn{3}{|l|}{ Median (CV) } \\
\hline Inhalation cut-off diameter & $15 \mu \mathrm{m}$ & see section 11.2 \\
\hline Inhalation rate & $23.1 \mathrm{l} / \mathrm{min}$ & \\
\hline \multicolumn{3}{|l|}{$\begin{array}{l}\text { Inhalation rate } \\
\text { Dermal }\end{array}$} \\
\hline Exposed area & $565 \mathrm{~cm}^{2}$ & $1 / 2$ area head female \\
\hline Amount upon head & $0.6 \mathrm{~g}$ & \\
\hline Exposure time & $960 \mathrm{~min}$ & $\begin{array}{l}\text { Estimate: } 16 \text { hours upon hair; at night, hair spray is } \\
\text { brushed out }\end{array}$ \\
\hline \multicolumn{3}{|l|}{ Hair styling, gel } \\
\hline Frequency & $358 \times /$ year & Mean: 0.59 a day SD: 0.57 (default: $358 \mathrm{x} /$ year $=0.98$ \\
\hline & & $\begin{array}{l}x / \text { day, i.e. calculated } 75^{\text {th }} \text { percentile from } 0.59 x / \text { day } \\
\text { and SD 0.57) }\end{array}$ \\
\hline \multicolumn{3}{|l|}{ Dermal, instant application } \\
\hline Exposed area no. 1 & $580 \mathrm{~cm}^{2}$ & $1 / 2$ area head \\
\hline Amount upon head & $0.3 \mathrm{~g}$ & $\begin{array}{l}10 \% \text { of } 2.9 \mathrm{~g} \text { product amount which is } 75^{\text {th }} \text { percentile } \\
\text { from mean: } 1.9 \mathrm{~g} \text {, SD: } 1.5\end{array}$ \\
\hline Exposure time & $1440 \mathrm{~min}$ & Estimate \\
\hline Exposed area no. 2 & $1010 \mathrm{~cm}^{2}$ & $1 / 2$ area hands $+1 / 2$ area head \\
\hline Amount product & $2.9 \mathrm{~g}$ & $75^{\text {th }}$ percentile from mean: $1.9 \mathrm{~g}$, SD: 1.5 \\
\hline Exposure time & $0.63 \mathrm{~min}$ & $75^{\text {th }}$ percentile from mean: $0.48 \mathrm{~min}, \mathrm{SD}: 0.22$ \\
\hline \multicolumn{3}{|l|}{ Hair styling, mousse } \\
\hline Frequency & $274 \times /$ year & Mean $0.41 x /$ day SD: 0.50 (default: $274 \mathrm{x} /$ year $=0.75$ \\
\hline & & $x /$ day i.e. $75^{\text {th }}$ percentile calculated from $0.41 \mathrm{x} /$ day, \\
\hline & & SD: 0.50$)$ \\
\hline \multicolumn{3}{|l|}{ Dermal, instant application } \\
\hline Exposed area no. 1 & $580 \mathrm{~cm}^{2}$ & $1 / 2$ area head \\
\hline Amount upon head & $0.3 \mathrm{~g}$ & $10 \%$ of $2.7 \mathrm{~g}$ product amount which is \\
\hline & & $75^{\text {th }}$ percentile from mean: 2.0, SD: 1.0 \\
\hline Exposure time & $1440 \mathrm{~min}$ & Estimate \\
\hline Exposed area no. 2 & $1010 \mathrm{~cm}^{2}$ & $1 / 2$ area hands $+1 / 2$ area head \\
\hline Amount product & $2.7 \mathrm{~g}$ & $75^{\text {th }}$ percentile from mean; $2.0 \mathrm{~g}, \mathrm{SD}: 1.0$ \\
\hline Exposure time & $0.67 \mathrm{~min}$ & $75^{\text {th }}$ percentile from mean: $0.4 \mathrm{~min}, \mathrm{SD}: 0.2$ \\
\hline \multicolumn{3}{|l|}{ Hair dye } \\
\hline Frequency & $10 \mathrm{x} /$ year & Studies range between 8-12 times per year \\
\hline \multicolumn{3}{|l|}{ Dermal, instant application } \\
\hline Exposed area & $580 \mathrm{~cm}^{2}$ & $1 / 2$ area head \\
\hline Amount product & $100 \mathrm{~g}$ & Studies range between 50 and $2 \times 50 \mathrm{~g}$ \\
\hline Exposure time & $40 \mathrm{~min}$ & $\begin{array}{l}\text { Estimation: } 5 \mathrm{~min} \text {. application and } 5 \mathrm{~min} \text {. } \\
\text { rinsing out; initial period } 20-40 \mathrm{~min}\end{array}$ \\
\hline \multicolumn{3}{|l|}{ Hair bleaching products ${ }^{b)}$} \\
\hline Frequency & 10x/year & \\
\hline \multicolumn{3}{|l|}{ Dermal, instant application } \\
\hline Exposed area & $580 \mathrm{~cm}^{2}$ & $1 / 2$ area head \\
\hline Amount product & $200 \mathrm{~g}$ & Estimate based on different products \\
\hline Exposure time & $45 \mathrm{~min}$ & Estimate \\
\hline
\end{tabular}




\begin{tabular}{|c|c|c|}
\hline & Default Value & Comments \\
\hline \multicolumn{3}{|l|}{ Hair perm ${ }^{\text {b) }}$} \\
\hline Frequency & $4 x /$ year & Estimate \\
\hline \multicolumn{3}{|l|}{ Dermal, instant application } \\
\hline Exposed area & $580 \mathrm{~cm}^{2}$ & $\begin{array}{l}1 / 2 \text { area head (Gloves are usually supplied with perm } \\
\text { lotion. It is assumed that gloves are used during its } \\
\text { application) }\end{array}$ \\
\hline \multicolumn{3}{|l|}{ Amount product } \\
\hline Perm lotion & $80 \mathrm{~g}$ & \\
\hline Fixing lotion & $80 \mathrm{~g}$ & \\
\hline \multicolumn{3}{|l|}{ Exposure time } \\
\hline Perm lotion & $40 \mathrm{~min}$ & \\
\hline Fixing lotion & $15 \mathrm{~min}$ & \\
\hline \multicolumn{3}{|c|}{ Soap liquid, solid: washing hands } \\
\hline Frequency & 1825 x/year & 3-6 x/day (default: $1825 \mathrm{x} / \mathrm{yr}=5 \mathrm{x} /$ day) \\
\hline \multicolumn{3}{|l|}{ Dermal, instant application } \\
\hline Exposed area & $860 \mathrm{~cm}^{2}$ & Area hands \\
\hline \multicolumn{3}{|l|}{ Amount upon skin dilution } \\
\hline Soap liquid & $3.0 \mathrm{~g}$ & Used amount $1.0 \mathrm{~g}$, dilution factor 3 \\
\hline Soap solid & $2.4 \mathrm{~g}$ & Used amount $0.8 \mathrm{~g}$, dilution factor 3 \\
\hline Weight fraction dilution & $W_{f} / 3$ & Estimate dilution factor 3 (wetting hands) \\
\hline Exposure time & $1 \mathrm{~min}$ & Estimate \\
\hline \multicolumn{3}{|l|}{ Soap liquid, solid: showering } \\
\hline Frequency & $329 \times /$ year & $1-2 x /$ day \\
\hline \multicolumn{3}{|l|}{ Dermal, instant application } \\
\hline Exposed area & $17500 \mathrm{~cm}^{2}$ & Total body area \\
\hline \multicolumn{3}{|l|}{ Amount upon skin dilution } \\
\hline Soap liquid & $26.1 \mathrm{~g}$ & Used amount $8.7 \mathrm{~g}$; dilution factor 3 \\
\hline Soap solid & $21.0 \mathrm{~g}$ & Used amount $7.0 \mathrm{~g}$; dilution factor 3 \\
\hline Weight fraction dilution & $W_{f} / 3$ & Estimate dilution factor 3 (use on wet skin) \\
\hline Exposure time & $4 \mathrm{~min}$ & \\
\hline \multicolumn{3}{|c|}{ Bath products: bath foam, bath salts and bath oil } \\
\hline Frequency & 104 x/year & 1-2 x/week; adults: $0.6-1.0 /$ week \\
\hline \multicolumn{3}{|l|}{ Dermal, instant application } \\
\hline Exposed area & $16340 \mathrm{~cm}^{2}$ & area body - area head \\
\hline Amount upon skin dilution & $16340 \mathrm{~g}$ & Estimate: $1 \mathrm{~cm}$ skin layer \\
\hline \multicolumn{3}{|l|}{ Weight fraction dilution } \\
\hline Bath foam & $W_{f} / 7000$ & Dilution factor: $1700 ; 17 \mathrm{~g}$ in $120 \mathrm{~L}$ \\
\hline Bath salts & $W_{f} / 4800$ & Dil. factor 4800 ; tablet of $25 \mathrm{~g}$ in $120 \mathrm{~L}$ \\
\hline Bath oil & $W_{f} / 13,000$ & $\begin{array}{l}\text { Dilution factor } 13000 ; 9 \mathrm{~g} \text { in } 120 \text { L3 i.e. } 10 \mathrm{ml} \text { bath oil } \\
\text { with density of } 0.9 \mathrm{~g} / \mathrm{cm}^{3}\end{array}$ \\
\hline \multicolumn{3}{|l|}{ Exposure time } \\
\hline \multicolumn{2}{|l|}{ Bath foam/ bath salts } & Estimate \\
\hline Bath oil & & Directions for use: $15-20 \mathrm{~min}$ \\
\hline Cream: Hand cream, facial & nd body lotion & \\
\hline Frequency & $730 \times /$ year & Estimate; based on different studies; $1-2 \times$ per day \\
\hline Dermal, instant application & & \\
\hline Exposed area & & \\
\hline Hand cream & $860 \mathrm{~cm}^{2}$ & Area hands \\
\hline Facial cream & $565 \mathrm{~cm}^{2}$ & $1 / 2$ area head female \\
\hline Body lotion & $15670 \mathrm{~cm}^{2}$ & Area body - area head female \\
\hline Amount product & & \\
\hline Hand cream & $1.7 \mathrm{~g}$ & \\
\hline Facial cream & $0.8 \mathrm{~g}$ & \\
\hline Body lotion & $8 \mathrm{~g}$ & Studies range between $7.5-8 \mathrm{~g}$ \\
\hline Exposure time & $720 \mathrm{~min}$ & 12 hours, estimate as a result of frequency \\
\hline Peeling gel (face) ${ }^{a)}$ & & \\
\hline Frequency & 104 x/year & $1-2 \times$ per week: directions for use \\
\hline Dermal & & \\
\hline Exposed area & $565 \mathrm{~cm}^{2}$ & $1 / 2$ area head female \\
\hline Amount product & $0.8 \mathrm{~g}$ & \\
\hline Exposure time & $5 \mathrm{~min}$ & Estimate \\
\hline Face packs: peel-off masks & d gel face pack & \\
\hline Frequency & 104 x/year & $1-2 \times$ per week \\
\hline Dermal, instant application & & \\
\hline Exposed area & $565 \mathrm{~cm}^{2}$ & $1 / 2$ area head female \\
\hline Amount product & $20 \mathrm{~g}$ & \\
\hline Exposure time & $20 \mathrm{~min}$ & \\
\hline
\end{tabular}




\begin{tabular}{|c|c|c|}
\hline & Default Value & Comments \\
\hline \multicolumn{3}{|c|}{ Body packs: mud bath /clay bath ${ }^{\text {a) }}$} \\
\hline Frequency & $4 \times /$ year & \\
\hline \multicolumn{3}{|c|}{ Dermal, instant application } \\
\hline Exposed area & $15670 \mathrm{~cm}^{2}$ & area body - area head \\
\hline Amount product & $416 \mathrm{~g}$ & \\
\hline Exposure time & $20 \mathrm{~min}$ & \\
\hline \multicolumn{3}{|c|}{ Skin whitening cream ${ }^{\text {a) }}$} \\
\hline Frequency & $91 \times /$ year & Estimate, an everyday use for three months is assumed \\
\hline \multicolumn{3}{|c|}{ Dermal, instant application } \\
\hline Exposed area & $565 \mathrm{~cm}^{2}$ & $1 / 2$ area head female \\
\hline Amount product & $5 \mathrm{~g}$ & \\
\hline Exposure time & $1440 \mathrm{~min}$ & 24 hours \\
\hline \multicolumn{3}{|c|}{ Facial make-up/foundation ${ }^{\text {a) }}$} \\
\hline Frequency & $365 \times /$ year & Estimate, once per day \\
\hline \multicolumn{3}{|c|}{ Dermal, instant application } \\
\hline Exposed area & $565 \mathrm{~cm} 2$ & $1 / 2$ area head female \\
\hline Amount product & $0.8 \mathrm{~g}$ & Estimate, face cream: $0.8 \mathrm{~g}$ \\
\hline Exposure time & $960 \mathrm{~min}$ & Estimate, 16 hours \\
\hline \multicolumn{3}{|c|}{ Cleansing lotion/make-up remover ${ }^{a)}$} \\
\hline Frequency & $730 \times /$ year & Studies range between $1-2$ times per day \\
\hline \multicolumn{3}{|l|}{ Dermal } \\
\hline Exposed area & $565 \mathrm{~cm}^{2}$ & $1 / 2$ area head female \\
\hline Amount product & $2.5 \mathrm{~g}$ & \\
\hline Exposure time & $5 \mathrm{~min}$ & Estimate \\
\hline \multicolumn{3}{|l|}{ Eye shadow ${ }^{\text {a) }}$} \\
\hline Frequency & $730 \times /$ year & $1-3 x /$ day \\
\hline \multicolumn{3}{|c|}{ Dermal, instant application } \\
\hline Exposed area & $24 \mathrm{~cm}^{2}$ & $\begin{array}{l}4 \times 3 \times 2=24 \mathrm{~cm}^{2} \text { (eyelids and the skin under the } \\
\text { eyebrows) }\end{array}$ \\
\hline Amount product & $0.01 \mathrm{~g}$ & \\
\hline Exposure time & $480 \mathrm{~min}$ & Estimate as a result of frequency \\
\hline \multicolumn{3}{|l|}{ Mascara ${ }^{a)}$} \\
\hline Frequency & 365 x/year & Once per day \\
\hline \multicolumn{3}{|c|}{ Dermal, instant application } \\
\hline Exposed area & $1.6 \mathrm{~cm}^{2}$ & Area of eyelashes \\
\hline Amount product & $0.025 \mathrm{~g}$ & \\
\hline Exposure time & $960 \mathrm{~min}$ & Estimate as a result of frequency \\
\hline \multicolumn{3}{|l|}{ Eyeliner ${ }^{\text {a) }}$} \\
\hline Frequency & 365 x/year & Once per day \\
\hline \multicolumn{3}{|c|}{ Dermal, instant application } \\
\hline Exposed area & $3.2 \mathrm{~cm}^{2}$ & $\begin{array}{l}4 \times 0.2 \times 4=3.2 \mathrm{~cm}^{2} \text { (eyeliner is applied as a thin line on } \\
\text { the eyelid just above or below the eyelashes) }\end{array}$ \\
\hline Amount product & $0.005 \mathrm{~g}$ & \\
\hline Exposure time & $960 \mathrm{~min}$ & Estimate as a result of frequency \\
\hline \multicolumn{3}{|c|}{ Eye make-up remover ${ }^{a)}$} \\
\hline Frequency & 365 x/year & 1-2 times per day \\
\hline \multicolumn{3}{|c|}{ Dermal, instant application } \\
\hline Exposed area & $50 \mathrm{~cm}^{2}$ & $5 \times 5 \times 2=50 \mathrm{~cm}^{2}$ (where eye make-up is applied) \\
\hline Amount product & $0.5 \mathrm{~g}$ & \\
\hline Exposure time & $5 \mathrm{~min}$ & Estimate \\
\hline \multicolumn{3}{|l|}{ Lipstick/ lip salve ${ }^{\text {a) }}$} \\
\hline Frequency & $1460 x /$ year & $2-6$ x/day; default: 4 x/day \\
\hline \multicolumn{3}{|l|}{ Oral, direct intake } \\
\hline Amount product & $0.01 \mathrm{~g}$ & \\
\hline Amount ingested & $0.01 \mathrm{~g}$ & It is assumed that the entire product is taken in orally \\
\hline
\end{tabular}




\begin{tabular}{|c|c|c|}
\hline & Default Value & Comments \\
\hline \multicolumn{3}{|l|}{ Nail polish ${ }^{\mathrm{a}, \mathrm{c}}$} \\
\hline Frequency & 156 x/year & 2-3 times a week \\
\hline \multicolumn{3}{|c|}{ Inhalation, evaporation from a constant surface } \\
\hline Exposure duration & $5 \mathrm{~min}$ & Estimate \\
\hline Product amount & $0.25 \mathrm{~g}$ & \\
\hline Application duration & $5 \mathrm{~min}$ & Estimate \\
\hline Room volume & $1 \mathrm{~m}^{3}$ & $\begin{array}{l}\text { The application of the nail polish takes place close to } \\
\text { the face. Therefore it is assumed that when the nail } \\
\text { polish is applied evaporation takes place in an initial } \\
\text { area of } 1 \mathrm{~m}^{3} \text {. }\end{array}$ \\
\hline Ventilation rate & $1 \mathrm{hr}^{-1}$ & Bedroom \\
\hline Release area & $19 \mathrm{~cm}^{2}$ & $\begin{array}{l}\text { It is assumed that a fingernail has an area of } 1 \times 1.5 \mathrm{~cm} \text {, } \\
\text { and then the total fingernail area is } 15 \mathrm{~cm}^{2} \text {. For the } \\
\text { contact of nail polish with the skin, a nail perimeter (i.e. } \\
\text { the two sides of the nail and the nail bed) of } 4 \mathrm{~cm} \text { is } \\
\text { assumed with a breadth of } 1 \mathrm{~mm} \text {. This gives an exposed } \\
\text { area of } 4 \mathrm{~cm}^{2} \text { for the skin around the fingernails, i.e. } 15+ \\
4 \mathrm{~cm}^{2}=19 \mathrm{~cm}^{2} \text {. }\end{array}$ \\
\hline Mol. weight matrix & $124 \mathrm{~g} / \mathrm{mol}$ & $\begin{array}{l}\text { The evaporation includes the parameter "molecular } \\
\text { weight matrix". This parameter is used to calculate the } \\
\text { relative vapour pressure of the component in question. } \\
\text { Nail polish contains } 75 \% \text { organic solvents; ethyl acetate } \\
(\mathrm{Mw}=88 \text { ), butyl acetate (Mw }=116) \text {, ethyl alcohol (Mw }= \\
46 \text { ) and toluene ( } \mathrm{Mw}=92 \text { ) in the ratio } 4: 3: 1: 740) \text {. The } \\
\text { molecular weight matrix is calculated as: }[4 / 15 * 88+ \\
3 / 15 * 116+1 / 15 * 46+7 / 15 * 92]: 0.75=124 \mathrm{~g} / \mathrm{mol} \text {. }\end{array}$ \\
\hline Inhalation rate & $23.1 \mathrm{l} / \mathrm{min}$ & \\
\hline \multicolumn{3}{|c|}{ Dermal, instant application } \\
\hline Exposed area & $4 \mathrm{~cm}^{2}$ & The skin around the fingernails \\
\hline Amount upon skin & $0.05 \mathrm{~g}$ & $0.25 \times 4 / 19=0.05 \mathrm{~g}$ \\
\hline Exposure time & $3360 \mathrm{~min}$ & Estimate as a result of frequency \\
\hline \multicolumn{3}{|c|}{ Nail polish remover ${ }^{a, c)}$} \\
\hline Frequency & 156 x/year & 2-3 times a week \\
\hline \multicolumn{3}{|c|}{ Inhalation, evaporation from a constant surface } \\
\hline Exposure duration & $5 \mathrm{~min}$ & Estimate \\
\hline Product amount & $0.5 \mathrm{~g}$ & Estimate \\
\hline Application duration & $5 \mathrm{~min}$ & Estimate \\
\hline Room volume & $1 \mathrm{~m}^{3}$ & See nail polish \\
\hline Ventilation rate & $1 \mathrm{hr}^{-1}$ & Bedroom \\
\hline Release area & $25 \mathrm{~cm}^{2}$ & $\begin{array}{l}\text { See nail polish; a larger area than nail polish is used } \\
\text { in order to remove the nail polish }\end{array}$ \\
\hline Mol. weight matrix & $75 \mathrm{~g} / \mathrm{mol}$ & $\begin{array}{l}\text { See nail polish; }(\mathrm{Mw}=88) \text {, butyl acetate }(\mathrm{Mw}=116) \\
\text { are assumed as solvents in the ratio } 13: 4: 140) \text {. The } \\
\text { molecule weight matrix is calculated as }[13 / 18 * 58+ \\
4 / 18 * 88+1 / 18 * 116]: 0.91=75 \mathrm{~g} / \mathrm{mol} \text { (see nail } \\
\text { polish scenario). }\end{array}$ \\
\hline Inhalation rate & $23.1 \mathrm{l} / \mathrm{min}$ & \\
\hline \multicolumn{3}{|c|}{ Dermal, instant application } \\
\hline Exposed area & $11 \mathrm{~cm}^{2}$ & $1.2 \times 0.2 \times 2=11 \mathrm{~cm}^{2}$ (the skin around the fingernails) \\
\hline Amount upon skin & $0.2 \mathrm{~g}$ & Estimate $(0.5 \times 11 / 26=0.2 \mathrm{~g})$ \\
\hline Exposure time & $5 \mathrm{~min}$ & Estimate \\
\hline \multicolumn{3}{|l|}{ Deodorant stick/ roller } \\
\hline \multicolumn{3}{|c|}{$\begin{array}{l}\text { Frequency } \\
\text { Dermal, instant application }\end{array}$} \\
\hline Exposed area & $100 \mathrm{~cm}^{2}$ & Estimate \\
\hline Amount product & $0.5 \mathrm{~g}$ & \\
\hline Exposure time & $1440 \mathrm{~min}$ & Estimate as a result of frequency \\
\hline
\end{tabular}




\begin{tabular}{|c|c|c|}
\hline & Default Value & Comments \\
\hline \multicolumn{3}{|l|}{ Deodorant spray } \\
\hline Frequency & $730 \times /$ year & Spray $1-3 \times$ per day \\
\hline \multicolumn{3}{|c|}{ Inhalation, spray model, spraying towards exposed person } \\
\hline Exposure duration & $5 \mathrm{~min}$ & Estimate: time in bathroom after spraying \\
\hline Spray duration & $0.17 \mathrm{~min}$ & Estimate; default: $0.17 \mathrm{~min}=10 \mathrm{sec}$ \\
\hline Room volume & $10 \mathrm{~m}^{3}$ & Bathroom \\
\hline Ventilation rate & $2 h^{-1}$ & Bathroom \\
\hline Cloud volume & $0.0625 \mathrm{~m}^{3}$ & See Table 11.2.1.1. \\
\hline Mass generation rate & $0.40 \mathrm{~g} / \mathrm{sec}$ & $\begin{array}{l}\text { Mean mass generation rate during the entire dura- } \\
\text { tion of spraying (full and nearly empty sprays) }\end{array}$ \\
\hline Airborne fraction & $1 \mathrm{~g} / \mathrm{g}$ & see section 11.2 \\
\hline Weight fraction non-volatile & $0.03 \mathrm{~g} / \mathrm{g}$ & \\
\hline Density non-volatile & $1.8 \mathrm{~g} / \mathrm{cm}^{3}$ & see section 11.2 \\
\hline Initial particle distribution & $10 \mu \mathrm{m}(0.3)$ & see section 11.2 \\
\hline \multicolumn{3}{|l|}{ Median (CV) } \\
\hline Inhalation cut-off diameter & $15 \mu \mathrm{m}$ & see section 11.2 \\
\hline Inhalation rate & $23.1 \mathrm{l} / \mathrm{min}$ & \\
\hline \multicolumn{3}{|l|}{ Dermal, instant application } \\
\hline Exposed area & $100 \mathrm{~cm}^{2}$ & Estimate \\
\hline Amount upon skin & $2.6 \mathrm{~g}$ & $\begin{array}{l}85 \% \text { of } 3.0 \mathrm{~g}-\text { it is assumed that only } 85 \% \text { ends up on } \\
\text { the skin }\end{array}$ \\
\hline Exposure time & $720 \mathrm{~min}$ & Estimate as a result of frequency \\
\hline \multicolumn{3}{|l|}{ Toothpaste, adults } \\
\hline Frequency & $730 \times /$ year & 1-2 per day \\
\hline \multicolumn{3}{|l|}{ Oral, direct intake } \\
\hline Amount ingested & $0.08 \mathrm{~g}$ & \\
\hline \multicolumn{3}{|c|}{ Toothpaste, children 2.5 years ${ }^{d)}$} \\
\hline Frequency & $730 \times /$ year & 1-2 times per day \\
\hline \multicolumn{3}{|l|}{ Oral, direct intake } \\
\hline Amount ingested & $0.53 \mathrm{~g}$ & \\
\hline \multicolumn{3}{|l|}{ Mouthwash } \\
\hline Frequency & $1460 x /$ year & $\begin{array}{l}\text { Studies range between } 1-5 \text { times per day; default } \\
1460 \mathrm{x} / \text { year }=4 \mathrm{x} / \text { day }\end{array}$ \\
\hline \multicolumn{3}{|l|}{ Oral, direct intake } \\
\hline Amount ingested & $1 \mathrm{~g}$ & \\
\hline \multicolumn{3}{|l|}{ Foot cream antiperspirant } \\
\hline Frequency & $730 \times /$ year & $1-2$ times per day; directions for use \\
\hline \multicolumn{3}{|l|}{ Dermal, instant application } \\
\hline Exposed area & $1170 \mathrm{~cm}^{2}$ & Area foot \\
\hline Amount product & $1.2 \mathrm{~g}$ & $\begin{array}{l}\text { An amount of } 1 \mathrm{mg} / \mathrm{cm}^{2} \text { is given for the use of cream in } \\
\text { general. The surface area of feet is } 1170 \mathrm{~cm}^{2} \text {. The } \\
\text { amount of cream used is calculated at } 1.2 \mathrm{~g} \text {. }\end{array}$ \\
\hline Exposure time & $720 \min$ & Estimate as a result of frequency \\
\hline \multicolumn{3}{|l|}{ Foot cream anti-fungal ${ }^{c}$} \\
\hline Frequency & $90 \times /$ year & $\begin{array}{l}\text { Directions for use: } 2 \text { times per day in approx. } 45 \text { days } \\
=90 \text { times per year. }\end{array}$ \\
\hline \multicolumn{3}{|l|}{ Dermal, instant application } \\
\hline Exposed area & $100 \mathrm{~cm}^{2}$ & \\
\hline Amount product & $0.1 \mathrm{~g}$ & $\begin{array}{l}\text { An amount of } 1 \mathrm{mg} / \mathrm{cm}^{2} \text { is given for the use of cream } \\
\text { in general. The surface area of feet is } 1170 \mathrm{~cm}^{2} \text {. It is } \\
\text { assumed that the cream is applied to } 100 \mathrm{~cm}^{2} \text { of the } \\
\text { skin of the feet. The amount of cream used is calcula- } \\
\text { ted at } 0.1 \mathrm{~g} \text {. }\end{array}$ \\
\hline Exposure time & $720 \mathrm{~min}$ & Estimate as a result of frequency \\
\hline
\end{tabular}




\begin{tabular}{|c|c|c|}
\hline & Default Value & Comments \\
\hline \multicolumn{3}{|l|}{ Eau de toilette spray } \\
\hline Frequency & 1095 x/year & $\begin{array}{l}\text { Studies range between } 1-5 \text { times per day; default: } 3 \\
\mathrm{x} / \text { day }\end{array}$ \\
\hline \multicolumn{3}{|l|}{ Inhalation } \\
\hline Exposure duration & $5 \mathrm{~min}$ & Estimate: time in bathroom after spraying \\
\hline Spray duration & $0.08 \mathrm{~min}$ & Estimate; $5 \mathrm{sec}$, based on values for trigger sprays \\
\hline Room volume & $10 \mathrm{~m}^{3}$ & Bathroom \\
\hline Ventilation rate & $2 h^{-1}$ & Bathroom \\
\hline Cloud volume & $0.0625 \mathrm{~m}^{3}$ & See Table 11.2.1.1. \\
\hline Mass generation rate & $0.14 \mathrm{~g} / \mathrm{sec}$ & $\begin{array}{l}\text { The mean mass generation rate is calculated from } \\
\text { the sprayed amount }(0.72 \mathrm{~g}) \text { and the total duration } \\
\text { of spraying }(5 \mathrm{sec}) \text {, which is } 0.14 \mathrm{~g} / \mathrm{sec} \text {. }\end{array}$ \\
\hline Airborne fraction & $0.2 \mathrm{~g} / \mathrm{g}$ & see section 11.2 \\
\hline Weight fraction non-volatile & $0.05 \mathrm{~g} / \mathrm{g}$ & \\
\hline Density non-volatile & $1.5 \mathrm{~g} / \mathrm{cm}^{3}$ & see section 11.2 \\
\hline Initial particle distribution & $50 \mu \mathrm{m}(0.6)$ & see section 11.2 \\
\hline \multicolumn{3}{|l|}{ Median (CV) } \\
\hline Inhalation cut-off diameter & $15 \mu \mathrm{m}$ & see section 11.2 \\
\hline Inhalation rate & $23.1 \mathrm{l} / \mathrm{min}$ & \\
\hline \multicolumn{3}{|l|}{ Dermal, instant application } \\
\hline Exposed area & $200 \mathrm{~cm}^{2}$ & $\begin{array}{l}\text { Estimate; the default value for the exposed body } \\
\text { surface is set at } 8 \times 25 \mathrm{~cm}^{2}=200 \mathrm{~cm}^{2} \text {. }\end{array}$ \\
\hline Amount upon skin & $0.61 \mathrm{~g}$ & $\begin{array}{l}\text { It is assumed that } 85 \% \text { of the eau de toilette ends up } \\
\text { on the skin, therefore } 0.61 \mathrm{~g}(0.85 \times 0.72 \mathrm{~g}) \text { ends up } \\
\text { on the skin. }\end{array}$ \\
\hline Exposure time & $320 \mathrm{~min}$ & Estimate as a result of frequency \\
\hline \multicolumn{3}{|l|}{ Perfume $^{e)}$} \\
\hline Frequency & $237 \times /$ year & Mean: 0.26 times per day \\
\hline \multicolumn{3}{|l|}{ Dermal, instant application } \\
\hline Exposed area & $100 \mathrm{~cm}^{2}$ & $\begin{array}{l}\text { As perfume contains more fragrance than eau de } \\
\text { toilette, the body surface onto which the perfume is } \\
\text { sprayed will be smaller: } 4 \times 25 \mathrm{~cm}^{2}=100 \mathrm{~cm}^{2} \text {. }\end{array}$ \\
\hline Amount upon skin & $0.20 \mathrm{~g}$ & $\begin{array}{l}0.23 \mathrm{~g} \text { per application - it is assumed that } 85 \% \text { of the } \\
\text { perfume ends up on the skin: } 0.20 \mathrm{~g} \text {. }\end{array}$ \\
\hline \multicolumn{3}{|l|}{ Aftershave $^{f)}$} \\
\hline Frequency & 365 x/year & $1-2 x /$ day \\
\hline \multicolumn{3}{|l|}{ Dermal, instant application } \\
\hline Exposed area & $305 \mathrm{~cm}^{2}$ & $1 / 4$ area head male \\
\hline Amount product & $1.2 \mathrm{~g}$ & \\
\hline Exposure time & $1440 \mathrm{~min}$ & Estimate as a result of frequency \\
\hline \multicolumn{3}{|l|}{ Shaving cream ${ }^{\mathrm{f})}$} \\
\hline Frequency & 365 x/year & $1 \mathrm{x} /$ day \\
\hline \multicolumn{3}{|l|}{ Dermal } \\
\hline Exposed area & $305 \mathrm{~cm}^{2}$ & $1 / 4$ area head male \\
\hline Amount product & $2 \mathrm{~g}$ & \\
\hline Exposure time & $5 \mathrm{~min}$ & Estimate \\
\hline \multicolumn{3}{|l|}{ Sunscreen lotion } \\
\hline Frequency & 75 x/year & $\begin{array}{l}2-3 \text { times per day for a period of } 2 \text { weeks/ year for the } \\
\text { use of sunscreen cream, and } 1 \text { week in the winter for } \\
\text { just the face. Based on this data the default value } \\
\text { assumes use } 3 \text { times per day over a period of } 25 \text { days. } \\
\text { (default: } 75 x / \text { year }=3 x / \text { day, } 25 \text { days/year) }\end{array}$ \\
\hline \multicolumn{3}{|l|}{ Dermal, instant application } \\
\hline Exposed area & $17500 \mathrm{~cm}^{2}$ & \\
\hline Amount product & $10 \mathrm{~g}$ & Cream $8 \mathrm{~g}$, lotion $10 \mathrm{~g}$ \\
\hline Exposure time & $150 \mathrm{~min}$ & Estimate \\
\hline
\end{tabular}




\begin{tabular}{|c|c|c|}
\hline & Default Value & Comments \\
\hline \multicolumn{3}{|c|}{ Baby products: baby cream/salve, baby oil and baby powdere, g) } \\
\hline Frequency & $730 \times /$ year & Estimate; default: $730 \mathrm{x} /$ year $=2 \mathrm{x} /$ day \\
\hline \multicolumn{3}{|l|}{ Dermal, instant application } \\
\hline Exposed area & $190 \mathrm{~cm}^{2}$ & $\begin{array}{l}\text { The default of the total body surface area of a child } \\
\text { of four and a half months is } 0.346 \mathrm{~m}^{2} \text {, and the } \\
\text { surface area of the torso is } 32.8 \% \text { of this body } \\
\text { surface. The estimate for the surface of a baby's } \\
\text { bottom is } 1 / 6 \text { of the exposed area of the torso, that } \\
\text { is } 1 / 6 \times 0.328 \times 3460 \mathrm{~cm}^{2}=190 \mathrm{~cm}^{2}\end{array}$ \\
\hline \multicolumn{3}{|l|}{ Amount product } \\
\hline Baby cream/salve & $0.27 \mathrm{~g}$ & \\
\hline Baby oil & $1.3 \mathrm{~g}$ & \\
\hline Baby powder & $0.8 \mathrm{~g}$ & \\
\hline Exposure time & $720 \mathrm{~min}$ & Estimate as a result of frequency \\
\hline \multicolumn{3}{|l|}{ Depilatory cream ${ }^{\text {a) }}$} \\
\hline Frequency & $17 \mathrm{x} /$ year & $\begin{array}{l}\text { Wax indicates that hairs will not grow back for } \\
\text { between } 3 \text { and } 6 \text { week depending on the hair } \\
\text { growth; } 17 x / \text { year }=1 \mathrm{x} / 3 \text { weeks }\end{array}$ \\
\hline \multicolumn{3}{|l|}{ Dermal, instant application } \\
\hline Exposed area & $5530 \mathrm{~cm}^{2}$ & Area female legs \\
\hline Amount product & $5.5 \mathrm{~g}$ & $1 \mathrm{mg} / \mathrm{cm}^{2}$; hair removal from legs \\
\hline Exposure time & $15 \mathrm{~min}$ & Studies range between $5-15 \mathrm{~min}$ \\
\hline \multicolumn{3}{|l|}{ Essential oil: massage } \\
\hline Frequency & 24 x/year & Estimate, $2 \times /$ month \\
\hline \multicolumn{3}{|l|}{ Dermal, instant application } \\
\hline Exposed area & $16340 \mathrm{~cm}^{2}$ & area body - area head \\
\hline Amount product & $8 \mathrm{~g}$ & $\begin{array}{l}\text { During a massage oil is rubbed into the body several } \\
\text { times, and it is assumed that the use of massage oil is } \\
\text { in the same order of magnitude as that of body } \\
\text { lotion; default value } 8 \mathrm{~g}\end{array}$ \\
\hline Exposure time & $30 \mathrm{~min}$ & Estimate \\
\hline \multicolumn{3}{|l|}{ Essential oil: bath } \\
\hline Frequency & $52 \times /$ year & Studies range between $0,6-2$ times per week \\
\hline \multicolumn{3}{|l|}{ Dermal, instant application } \\
\hline Exposed area & $16340 \mathrm{~cm}^{2}$ & area body - area head \\
\hline Amount upon skin dilution & $16340 \mathrm{~g}$ & $\begin{array}{l}\text { Estimate: } 1 \mathrm{~cm} \text { skin layer; As default value it is } \\
\text { assumed that } 10 \mathrm{ml} \text { of bath product ( } 10 \mathrm{ml} \text { oil with } \\
\text { density of } 0.9 \mathrm{~g} / \mathrm{cm}^{3} \text { i.e. } 9 \mathrm{~g} \text { ) with } 30 \% \text { essential oils is } \\
\text { added to } 120 \text { litres of bath water }\end{array}$ \\
\hline Weight fraction dilution & $W_{f} / 13000$ & $\begin{array}{l}\text { Dilution factor } 13000: 9 \mathrm{~g} \text { in } 120 \text { litre }(10 \mathrm{ml} \text { oil with } \\
\text { density of } 0.9 \mathrm{~g} / \mathrm{cm}^{3} \text { ) }\end{array}$ \\
\hline Exposure time & $20 \mathrm{~min}$ & Directions for use: $15-20 \mathrm{~min}$ \\
\hline \multicolumn{3}{|l|}{ Essential oil: air freshener } \\
\hline Frequency & $168 \times /$ year & \\
\hline \multicolumn{3}{|c|}{ Inhalation, evaporation, constant rate } \\
\hline Exposure duration & $240 \min$ & $4 \mathrm{hrs}$ \\
\hline Product amount & $1.08 \mathrm{~g}$ & $\begin{array}{l}1 \text { drop oil is about } 50 \mu \text { which equals } 45 \mathrm{mg} \text { (density } \\
0.9 \mathrm{~g} / \mathrm{cm}^{3} \text { ); } 24 \text { drops oil equal } 1.08 \mathrm{~g}\end{array}$ \\
\hline Room volume & $58 \mathrm{~m}^{3}$ & Living room \\
\hline Ventilation rate & $0.5 \mathrm{~h}^{-1}$ & Living room \\
\hline Emission duration & $180 \min$ & Estimate \\
\hline Inhalation rate & $23.1 \mathrm{l} / \mathrm{min}$ & \\
\hline \multicolumn{3}{|l|}{ Face paint, adult } \\
\hline Frequency & $6 x /$ year & Estimate \\
\hline \multicolumn{3}{|l|}{ Dermal, instant application } \\
\hline Exposed area & $580 \mathrm{~cm}^{2}$ & $1 / 2$ area head \\
\hline Amount product & $1.7 \mathrm{~g}$ & $\begin{array}{l}\text { With regard to the amount of face paint used, it is } \\
\text { assumed that this is } 3 \text { times as large as the amount } \\
\text { used for a general cream, that is } 3 \mathrm{mg} / \mathrm{cm}^{2}\end{array}$ \\
\hline Exposure time & $480 \mathrm{~min}$ & Estimate, 8 hours \\
\hline
\end{tabular}




\begin{tabular}{|c|c|c|}
\hline & Default Value & Comments \\
\hline \multicolumn{3}{|l|}{ Face paint, child ${ }^{\mathrm{h})}$} \\
\hline Frequency & $12 \times /$ year & Estimate \\
\hline \multicolumn{3}{|c|}{ Dermal, instant application } \\
\hline Exposed area & $475 \mathrm{~cm}^{2}$ & $1 / 2$ area head \\
\hline Amount product & $1.4 \mathrm{~g}$ & $\begin{array}{l}\text { With regard to the amount of face paint used, it is } \\
\text { assumed that this is } 3 \text { times as large as the amount } \\
\text { used for a general cream, that is } 3 \mathrm{mg} / \mathrm{cm}^{2}\end{array}$ \\
\hline Exposure time & $480 \mathrm{~min}$ & Estimate, 8 hours \\
\hline
\end{tabular}

a) Only females - having an assumed body weight of $61 \mathrm{~kg}$.

b) Gloves are usually supplied with the product. It is assumed that gloves are used during its application.

c) For the dermal exposure the amount that is applied to the nail is not important, only the amount that is applied to the skin.

d) Assuming a body weight of $12.5 \mathrm{~kg}$.

e) Data taken from the Exposure Factors Handbook, US-EPA (1997).

f) Only males - having an assumed body weight of $74 \mathrm{~kg}$.

g) Assuming a body weight of $6.21 \mathrm{~kg}$ for a 4.5 months old child.

h) Assuming a body weight of $16.3 \mathrm{~kg}$ for a 4.5 years old child.

Source: Modified from the Cosmetics Fact Sheet, RIVM (2006b).

Note: All references are cited in the Cosmetics Fact Sheet, RIVM (2006b).

\subsubsection{Cleaning products}

In the Cleaning Product Fact Sheet, RIVM (2006c) cleaning products are classified into 36 product categories, which are drawn up according to the type of product. As in the Cosmetics Fact Sheet, RIVM (2006b) various default exposure models are chosen and filled in with default parameter values. These models are thoroughly described in the Fact Sheets, but will only shortly be mentioned here, when necessary. The Technical Notes for Guidance on Human Exposure to Biocidal Products (TNsG) has provided most of the data used in this fact sheet (TNsG, 2007).

Most cleaning products are ready for use. However, some of the cleaning products require preparation before they can be used; so-called mixing and loading has to be applied first. During the mixing and loading process additional exposure may occur.

The parameters during the spraying process is mentioned in the previous section, however, there are some differences when spraying with cosmetics and cleaning products. Cosmetics sprays are used directly towards the consumer whereas cleaning products are used to clean different surfaces away from the consumer. Here, only the parameters that differ from the spraying process for cosmetics are mentioned.

- Spray duration and exposure duration: The duration of spraying and rinsing/cleaning is directly related to the size of the cleaned area. The default values for spraying and for cleaning are set at $14.3 \mathrm{sec} / \mathrm{m}^{2}$ and $110 \mathrm{sec} / \mathrm{m}^{2}$, respectively, based on a pilot study (RIVM, 2006c) 
- Mass generation rate: The default value for mass generation rate is set at $0.78 \mathrm{~g} / \mathrm{sec}$ and it is used for the following trigger sprays: allpurpose spray, bathroom spray, glass spray and oven spray. This default value is based on different studies (RIVM, 2006c)

- Initial particle distribution: This parameter is described under "cosmetics", however, default values for initial particle distribution for cleaning sprays are given. For aerosol spray cans, the default is a lognormal distribution with median $25 \mu \mathrm{m}$, coefficient of variation 0.4 . For trigger sprays, the default is a lognormal distribution with median $100 \mu \mathrm{m}$, coefficient of variation 0.6 (RIVM, 2006c)

- Airborne fraction: The default values for airborne fraction are identical for cosmetic sprays and cleaning sprays

- Density: See under "cosmetics"

- Inhalation cut-off diameter: See under "cosmetics"

In the Cleaning Product Fact Sheet, RIVM (2006c), dermal exposure of the user during application is calculated based on the "constant rate" model from ConsExpo. The Technical Notes for Guidance on Human Exposure to Biocidal Products (TNsG, 2007) has provided the data for this model. For the "constant rate" model the parameter "contact rate" is required. The contact rate is the rate at which the product is applied to the skin, in weight per time unit. The "constant rate" model is used for all spray applications. The following parameters are used in the "constant rate" model:

- Contact rate aerosol spray cans: The dermal exposure on hands and forearms ranges from 1.7 to $156 \mathrm{mg} / \mathrm{min}$ with a $75^{\text {th }}$ percentile of $64.7 \mathrm{mg} / \mathrm{min}$. The dermal contact rate for legs, feet and face ranges from 17 to $45.2 \mathrm{mg} / \mathrm{min}$ with a $75^{\text {th }}$ percentile of $35.7 \mathrm{mg} / \mathrm{min}$. Using these data, the default value for contact rate aerosol spray cans is set at $100 \mathrm{mg} /$ minute (RIVM, 2006c)

- Contact rate trigger sprays: The dermal exposure on hands and forearms ranges from 3 to $68.2 \mathrm{mg} / \mathrm{min}$ with a $75^{\text {th }}$ percentile of 36.1 $\mathrm{mg} / \mathrm{min}$. The dermal contact rate for legs, feet and face ranges from 1.9 to $12.4 \mathrm{mg} / \mathrm{min}$ with a $75^{\text {th }}$ percentile of $9.7 \mathrm{mg} / \mathrm{min}$. Using these data, the default value for contact rate for trigger sprays is set at 46 $\mathrm{mg} /$ minute

In Table 11.4, an overview is given for the default values of aerosol spray cans and trigger sprays and in Table 11.5, default parameters that are used repeatedly in the Product Fact Sheet, RIVM (2006c) are provided. Table 11.6 presents default values on cleaning products for nonprofessional use as described in the Cleaning Product Fact Sheet, RIVM (2006c). 
Table 11.4: Overview of Default Values of Aerosol Cans and Trigger Sprays

\section{Default Value}

Aerosol Spray Can

Inhalation, spray model

Mass generation rate

Airborne fraction

Density non-volatile

$1.5 \mathrm{~g} / \mathrm{sec}$

Initial particle distribution; median (CV)

$1 \mathrm{~g} / \mathrm{g}$

$1.8 \mathrm{~g} / \mathrm{cm}^{3}$

Inhalation cut-off diamete

$25 \mu \mathrm{m}(0.4)$

$15 \mu \mathrm{m}$

Dermal, constant rate

Contact rate

$100 \mathrm{mg} / \mathrm{min}$

Trigger Sprays

Inhalation, spray model

Mass generation rate

Airborne fraction

Density non-volatile

$0.78 \mathrm{~g} / \mathrm{sec}$

$0.2 \mathrm{~g} / \mathrm{g}$

$1.8 \mathrm{~g} / \mathrm{cm}^{3}$

Initial particle distribution; median (CV) $100 \mu \mathrm{m}(0.6)$

Inhalation cut-off diameter $\quad 15 \mu \mathrm{m}$

Dermal, constant rate

Contact rate

$46 \mathrm{mg} / \mathrm{min}$

Source: Modified from the Cleaning Product Fact Sheet, RIVM (2006c).

Table 11.5: Default Values Used Repeatedly in the Fact Sheet- Supplement to Table 11.6

Default value

Ventilation rates (inhalation model - spraying and evaporation)

Bathroom/toilet

$2.0 \mathrm{~h}^{-1}$

Kitchen

$2.5 \mathrm{~h}^{-1}$

Living room

$0.5 \mathrm{~h}^{-1}$

Garage

$1.5 h^{-1}$

Non-specified room

$0.6 \mathrm{~h}^{-1}$

Room temperature (inhalation model)

$20{ }^{\circ} \mathrm{C}$

Room volumes (inhalation model - spraying and evaporation)

Bathroom

Toilet

$10 \mathrm{~m}^{3}$

$2.5 \mathrm{~m}^{3}$

Kitchen

$15 \mathrm{~m}^{3}$

Living room

Garage

$58 \mathrm{~m}^{3}$

Non-specified room

$34 \mathrm{~m}^{3}$

Standard room height (inhalation model - spraying and evaporation)

Uptake fraction (inhalation, dermal and oral uptake)

$20 \mathrm{~m}^{3}$

Inhalation rate (inhalation)

These parameters are used in Table 11.2.2.3. unless else is stated.

Source: The Cleaning Product Fact Sheet, RIVM (2006c). 


\section{Laundry powder: filling the washing machine}

Frequency

365 year $^{-1}$

Exposure duration

Product amount

Room volume

Laundry powder: hand washing

Frequency

Dermal instant application

Exposed area

Weight fraction dilution

Product amount dilution

Concentration dilution

Exposure time

$0.25 \min$

$2.7 * 10^{-7} \mathrm{~g}$

$1 \mathrm{~m}^{3}$

104 year $^{-1}$

$1900 \mathrm{~cm}^{2}$

$0.01 * \mathrm{~W}_{\mathrm{f}}$

$19 \mathrm{~g}$

$0.01 * W_{f} g / c$

$10 \mathrm{~min}$

Laundry powder: residues on clothing

Frequency

Leachable fraction

365 year $^{-1}$

$0.003 * W_{f}$

Product amount

Skinct amount

1000

\section{Laundry liquid: filling the washing machine}

Frequency 365 year
Inhalation, evaporation from constant surface

Exposure duration $\quad 0.75 \mathrm{~min}$

Product amount

$500 \mathrm{~g}$

Room volume

Release area

Application duration

Mol. weight matrix

Dermal, instant application

Exposed area

Product amount

Exposure time

$0.002 \mathrm{~m}^{2}$

$0.3 \mathrm{~min}$

$90 \mathrm{~g} / \mathrm{mol}$

$215 \mathrm{~cm}^{2}$

$0.010 \mathrm{~g}$
Based on different studies it is estimated that a consumer typically fills the washing machine 7 times a week

Estimate is based on one study

A cup containing 200 gram of washing powder can generate $0.27 \mu \mathrm{g}$ dust.

"Room volume" is interpreted here as "personal volume": a small area of $1 \mathrm{~m}^{3}$ around the user.

Estimate: 2 week $^{-1}$

Area hands and forearms

The concentration of laundry detergent for the hand-wash is $0.1 \%$ to $1 \%$. Worst-case, the weight fraction of the diluted detergent is $1 \%$ of the used detergent powder

is assumed that not the total amount of diluted product is in contact with the skin but only a layer around the exposed skin. It has been estimated that the thickness of a product layer on the skin at $0.01 \mathrm{~cm}$. The exposed area is $1900 \mathrm{~cm}^{2}$; thus, the amount of diluted product is $19 \mathrm{~cm}^{3}$ or 19

Diffusion trough skin. Density water $1 \mathrm{~g} / \mathrm{cm}^{3}$

It is assumed that one wears clothes, e.g. underwear, nightclothes, blouses, trousers and socks, every day during 24 hours.

The leachable fraction is the relative amount of chemical which can leach from a product $(\mathrm{g} / \mathrm{g})$ i.e. the fraction of deposits of the detergent which can leach from textile. The leachable weight fraction depends on the composition of the detergent, the compound in question and the type of textile. Because there are no data for the amount of detergent residues leachin from the textile, it is assumed that $50 \%$ is leachable. The calculation for the leachable weight fraction is as follows: $6 \times 10^{-3} \mathrm{~g} / \mathrm{g} \times 0.5 \times \mathrm{W}_{\mathrm{f}}=3 \times 10^{-3} \times \mathrm{W}_{\mathrm{f}}$

The total weight of fabric i.e. average weight product during the day that is worn on the body is estimated at $1000 \mathrm{gram}$.

or the calculation of the skin-contact factor (i.e. the part of the product that is actually in contact with bare skin) it is assumed that $1 / 2$ of the clothes are in direct contact with the skin (e.g. underwea Fkin $=1$ ) and $1 / 2$ contacts on and off the skin (e.g. blouses, Fskin $=0.6$ ). The skin-contact factor then becomes $0.5 \times 1+0.5 \times 0.6=0.8$

The frequency ranges from 2.8-10 times a week. The default value is 7 times a week.

Studies on exposure duration for filling the dishwasher range from 6 to 45 seconds with a mean of $23 \mathrm{~s}$. The calculated $75^{\text {th }}$ percentile is $34.6 \mathrm{sec}$ or $0.58 \mathrm{~min}$. The maximal duration of $45 \mathrm{~s}$ 0.75 min is set as defaut var

aporated amount of compound from the product. It is not the used product amount but half of the bottle content. For a one-litre bottle the averaged mount liquid in the bottle is estimated at $500 \mathrm{~g}\left(\right.$ density $1 \mathrm{~g} / \mathrm{cm}^{3}$ ), which is set as default value.

See "Laundry powder: filling the washing machine"

.

The fraction of water is estimated at $20 \%$; the molecular weight matrix becomes $18 \mathrm{~g} / \mathrm{mol} / 0.2=90 \mathrm{~g} / \mathrm{mol}$

One palm: $1 / 4$ area hands

Estimate. For comparison, one small drop liquid is about $0.02 \mathrm{ml}$ or 0.02

l.e. exposure duration 


\section{Spray spot remover: spraying ${ }^{\text {a) }}$}

Frequency

Inhalation, spray model

Spray duration

Exposure duration

Mass generation rat

Airborne fraction

Weight fraction non-volatile

Density non-volatile

Initial particle distribution Median (CV)

Inhalation cut-off diameter

Oral uptake fraction

Dermal, constant ra

Release duration

Spray spot remover: washing

Frequency

Dermal, instant application

Exposed area

Weight fraction dilution

Product amount dilution

Concentration dilution

Exposure time

Liquid spot remover/pastes

Frequency

Dermal, instant application

Exposed area

Weight fraction dilution

Concentration dilution

Exposure time

128 year $^{-1}$

$0.05 \mathrm{~min}$

$10 \mathrm{~min}$

$10 \mathrm{~min}$

$1.5 \mathrm{~g} / \mathrm{sec}$

$0.2 \mathrm{~g} / \mathrm{g}$

$1.8 \mathrm{~g} / \mathrm{cm}^{3}$

$1.8 \mathrm{~g} / \mathrm{cm}^{3}$

$15 \mu \mathrm{m}$

$46 \mathrm{mg} / \mathrm{min}$

$0.05 \mathrm{~min}$

128 year $^{-1}$

$430 \mathrm{~cm}^{2}$

$0.1 * \mathrm{~W}^{\prime}$

$2.0 \mathrm{~g}$

$0.1 * \mathrm{~W}_{\mathrm{f}} \mathrm{g} / \mathrm{cm}^{3}$

$10 \mathrm{~min}$

128 year $^{-1}$

$430 \mathrm{~cm}^{2}$

$0.1 * W_{f}$

$0.65 \mathrm{~g} / 2.5 \mathrm{~g}$

$0.1 * W_{f} g / \mathrm{cm}^{3}$

$10 \mathrm{~min}$

426 year $^{-1}$

liquid: mixing and loading

Frequency

Inhalation, evaporation from constant surface

Exposure duration

Product amount

Room volume

$0.75 \mathrm{~min}$

Release area

Application duration

Dermal, instant application

Exposed area

Product amount

Exposure time

$0.002 \mathrm{~m}^{2}$

$0.3 \mathrm{~min}$

$36 \mathrm{~g} / \mathrm{mol}$

$215 \mathrm{~cm}^{2}$

$0.010 \mathrm{~g}$
Studies range from 0.06 to 0.82 a day, with a weighted mean of 0.35 a day.

The default value for the mass generation rate of a spray is $1.5 \mathrm{~g} / \mathrm{sec}$. For spray spot remover, the used amount was $3.9 \mathrm{gram}$ per task. Therefore, it is calculated, that the period of active spraying is $3.9 \mathrm{~g} / 1.5 \mathrm{~g} / \mathrm{s}=2.6$ seconds. The default value for spray duration is set at 3 seconds or $0.05 \mathrm{~min}$.

Estimate

See above

Pre-wash soil and stain removers contain $5-15 \%$ surfactants. Based on the general composition, it is assumed that the non-volatile part in spray spot removers is about $10 \%$ - the default

value weight fraction non-volatile is set at $0.1 \mathrm{~g} / \mathrm{s}$

See above

See above

See above

Potential dose

See above

l.e. spray duration

Studies range from 0.06 to 0.82 a day, with a weighted mean of 0.35 a day.

$1 / 2$ area hands

For spray spot removers, the amount per task is $3.9 \mathrm{gram}$. For estimating dermal exposure, it is assumed that $5 \%$ of the amount contacts the skin, i.e. $0.2 \mathrm{~g}$. Taken the dilution into account (10 times), the default value for the diluted product amount is set at $2.0 \mathrm{~g}$.

Diffusion trough skin. Density water $1 \mathrm{~g} / \mathrm{cm}^{3}$

Estimate

Studies range from 0.06 to 0.82 a day, with a weighted mean of 0.35 a day

$1 / 2$ area hands

Dilution 10x

For liquid spot removers, the amount per task is $1.3 \mathrm{gram}$. Assuming that $5 \%$ contacts the skin and taking the dilution of 10 times into account, the default value is set at $0.65 \mathrm{~g}$ diluted product/For pastes, the default value is $5 \%$ of $5 \mathrm{gram}$ i.e. $0.25 \mathrm{~g}$. Assuming that $5 \%$ contacts the skin and taking the dilution of 10 times into account, the default value is set at $2.5 \mathrm{~g}$ diluted product

Dffusion trough skin. Density water $1 \mathrm{~g} / \mathrm{cm}^{3}$

Estimate

Studies range between 3-21 times a week and a mean value of 0.63 per day with a 75-percentile of 1.17 day per day $(n=45)$, which results in a frequency of 426 per year.

See "Laundry liquid: filling the washing machine"

See "Laundry liquid: filling the washing machine"

See "Laundry powder: filling the washing machin"

ee "Iaundry powder: filling the washing machin"

Estimation

Assuming the product contains $50 \%$ water, the molecular weight matrix is $18 \mathrm{~g} / \mathrm{mol} / 0.5=36 \mathrm{~g} / \mathrm{mol}$

One palm: $1 / 4$ area hands

Estimate

e. exposure duration 
Dishwashing liquid regular: hand-wash ${ }^{\text {b) }}$

Frequency

426 year $^{-1}$

Inhalation, evaporation from constant surface

Exposure duration

Product amount dilution

$60 \mathrm{~min}$

Weight fraction dilution

$\mathrm{W}_{\mathrm{f}} / 714$

Release area

Application duration

Temperature

$0.15 \mathrm{~m}^{2}$

$16 \mathrm{~min}$

$45^{\circ} \mathrm{C}$

Mol. weight matrix

Dermal, instant application

Exposed area

Weight fraction dilution

Product amount dilutio

Concentration dilution

Exposure time

Dishwashing liquid: residues on dinnerw

Frequency

Oral, direct intake

Amount ingested

\section{$18 \mathrm{~g} / \mathrm{mol}$}

$860 \mathrm{~cm}^{2}$

$\mathrm{W}_{\mathrm{f}} / 714$

$3.6 \mathrm{~g}$

$8.6 \mathrm{~g}$
$\mathrm{~W}_{\mathrm{f}} / 714 \mathrm{~g} / \mathrm{cm}$

$16 \mathrm{~min}$

365 year $^{-1}$

365 year $^{-1}$

$4.2 * 10^{-4} \mathrm{~g}$

filling the dishwashing machine

Frequency

35 year

Inhalation, evaporation from constant surface

Exposure duration

Product amount

Room volume

$0.75 \mathrm{~min}$

$0.75 \mathrm{~min}$

Application duration

$1 \mathrm{~m}^{3}$

$1 \mathrm{~m}^{3}$
$0.002 \mathrm{~m}^{2}$

$0.002 \mathrm{~m}^{2}$

Dermal, instant application

Exposed area

Product amount

$60 \mathrm{~g} / \mathrm{mol}$

$215 \mathrm{~cm}^{2}$
$0.010 \mathrm{~g}$

$0.75 \mathrm{~min}$
See "Dishwashing liquid: mixing and loading"

Estimate

This parameter is to limit the evaporated amount of compound from the product. The amount is calculated by multiplying the release area of $1500 \mathrm{~cm}^{2}$ with a water height of about $10 \mathrm{~cm}$ resulting in an amount of $15,000 \mathrm{~cm}^{3}$ water i.e. 15,000 gram.

Studies range between $3-10 \mathrm{~g}$ with a $75^{\text {th }}$ percentile of $7 \mathrm{gram}$ (per 5 litres). This gives a concentration of $1.4 \mathrm{~g} / \mathrm{l}$. The fraction in the washing-up bowl is $7 \mathrm{~g} / 5000 \mathrm{~g}=1.4 \times 10^{-3}$ of the dishwashing product. This means a dilution of about 714 times; the weight fraction of the solution is the weight fraction of the product divided by the dilution factor ( $\mathrm{W}^{\mathrm{f}} / 714$ ). When doing the dishes, the release area equals the surface of the sink. In a study the $75^{\text {th }}$ percentile of the surface of the sinks was calculated as $1453 \mathrm{~cm}^{2}$. The default value is set at $0.15 \mathrm{~m}^{2}$

In one study, the duration of contact per event was 11 min with a $75^{\text {th }}$ percentile of $16 \mathrm{~min}$, which is set as default value for application duration (inhalation exposure) and for exposure time (dermal exposure).

Initially, the temperature of the dishwater is high, approximately $60^{\circ} \mathrm{C}$ and while doing the dishes, the temperature will decrease. The most frequently used dishwashing temperatures are in the range of $40-45^{\circ} \mathrm{C}$. Therefore, the default value for the "average" temperature is estimated at $45^{\circ} \mathrm{C}$.

$40-45^{\circ} \mathrm{C}$. Therefore, the default value for the "average" temperat
Matrix is water; see "Dishwashing liquid: mixing and loading"

Area hands

See under "Inhalation"

Skin layer: $0.01 \mathrm{~cm}$

Density water $1 \mathrm{~g} / \mathrm{cm}^{3}$

I.e. application duration

Estimate - once a day.

According to one study, the value for amount of water left on dishes is $5.5 \times 10-5 \mathrm{ml} / \mathrm{cm}^{2}$ and the value for the area of dishes in daily contact with food is $5400 \mathrm{~cm}^{2}$. The concentration of the dishwashing water is $1.4 \mathrm{~g} / \mathrm{l}$. Therefore, the ingested product amount is $5.5 \times 10-5 \mathrm{ml} / \mathrm{cm}^{2} \times 5400 \mathrm{~cm}^{2} \times 1.4 \mathrm{mg} / \mathrm{ml}=0.4158 \mathrm{mg}$.

It is estimated that the use is 2 times per 3 weeks

See "Laundry liquid: filling the washing machine"

See "Laundry liquid: filling the washing machine"

See "Laundry powder: filling the washing machine"

See "Laundry powder: filling the washing machine"

Estimation

The fraction of water in polishing liquid is estimated at 0.3 ; so, the molecular weight fraction is $18 \mathrm{~g} / \mathrm{mol} / 0.3=60 \mathrm{~g} / \mathrm{mol}$

One palm: $1 / 4$ area hands

Estimate

e. exposure duration 


\section{Default Value Comments}

\section{All-purpose cleaner liquid: mixing and loading}

Frequency

104 year $^{-1}$

Ihalation, evaporation from constant surface

Exposure duration $\quad 0.75 \mathrm{~min}$

Product amount

Room volume
Release area

Release area

$1 \mathrm{~m}^{3}$

$0.002 \mathrm{~m}^{2}$

$0.3 \mathrm{~min}$

$22 \mathrm{~g} / \mathrm{mo}$

Dermal, instant application

Exposed area

Product amount

$215 \mathrm{~cm}^{2}$

$215 \mathrm{~cm}^{2}$

$0.010 \mathrm{~g}$

Exposure time

Frequency

104 year $^{-1}$

Inhalation, evaporation from increasing area

Exposure duration $240 \mathrm{~min}$

Product amount dilution

Weight fraction dilution $\quad W_{f} / 80$

\section{Release area}

Application duratio

Mol. weight matrix

Dermal, instant application

Exposed area

Weight fraction dilution

Product amount dilutio

Concentration dilution

Exposure time

$W_{1 / 80}$

$10 \mathrm{~m}^{2}$

$10 \mathrm{~m}^{2}$
$20 \mathrm{~min}$

$20 \mathrm{~min}$

$1900 \mathrm{~cm}^{2}$

$1900 \mathrm{~cm}^{2}$

$W_{\mathrm{f}} / 80$

$19 \mathrm{~g}$

$20 \mathrm{~min}$

kitchen working top

\section{All-purpose spray cleaner: spraying the kitchen working top ${ }^{\text {b) }}$}

Frequency

365 year $^{-1}$

Inhalation, spray model

Spray duration

Exposure duration

Mass generation rate

Weight fraction non-volatile

Weight fraction non-

Density non-volatile

Initial particle distribution Median (CV)

Inhalation cut-off diameter

Dermal, constant rate

Contact rate

$0.41 \mathrm{~min}$

$60 \mathrm{~min}$

$0.78 \mathrm{~g} / \mathrm{sec}$

$0.2 \mathrm{~g} / \mathrm{g}$

$0.05 \mathrm{~g} / \mathrm{g}$

$1.8 \mathrm{~g} / \mathrm{cm}^{3}$

$15 \mu \mathrm{m}$

$46 \mathrm{mg} / \mathrm{min}$

$0.41 \mathrm{~min}$
Based on two studies, the mean use is two times per week

See "Laundry liquid: filling the washing machine"

See "Laundry liquid: filling the washing machine"

See "Laundry powder: filling the washing machine"

See "Laundry powder: filling the washing machine"

Estimation

The fraction of water in the product is estimated at 0.8 . For the molecular weight matrix, the default value is $18 \mathrm{~g} / \mathrm{mol} / 0.8=22 \mathrm{~g} / \mathrm{mol}$

One palm: $1 / 4$ area hands

Estimate

e. exposure duration

See "All-purpose cleaner liquid: mixing and loading"

Default

In a limited experiment $40 \mathrm{ml}$ suds was spread on a surface of $1^{\mathrm{m}} 2$. The area was quite soaked; therefore, it is assumed that the amount left on the surface is not exceeding $40 \mathrm{ml} \mathrm{per} \mathrm{m}^{2}$ The amount of suds for an area of $10 \mathrm{~m}^{2}$ is set at $400 \mathrm{ml}$ or $400 \mathrm{~g}$.

Studies range between $25-110 \mathrm{~g}$ per 5 litres of wash volume. Based on the $75^{\text {th }}$ percentile, a total of $63 \mathrm{~g}$ per 5 litres is used as default, which gives a dilution of 80 times. The weight fraction of the suds is the weight fraction of the product divided by the dilution factor $\left(W_{f} / 80\right)$.

"Product amount dilution"

Based on two studies

Matrix is water; see "Dishwashing liquid: mixing and loading"

Area hands and forearms

see "Inhalation"

Dension trough skin. Density water $1 \mathrm{~g} / \mathrm{cm}^{3}$

e. application duration

Estimate: 1 day $^{-1}$

One study has calculated the $75^{\text {th }}$ percentile for the spraying as $14.3 \mathrm{sec}$. per $\mathrm{m}^{2}$. For an area of $1.71 \mathrm{~m}^{2}$, the spraying time is $0.41 \mathrm{~min}$ Estimate

ee Table 11.2.2.1

Based on the general composition, it is assumed that the non-volatile part in all-purpose spray cleaners is about $5 \%$; the default value weight fraction non-volatile is set at $0.05 \mathrm{~g} / \mathrm{g}$.

See Table 11.2.2.1.

See Table 11.2.2.1.

See Table 11.2.2.1.

l.e. spray duration 


\section{Default Value Comments}

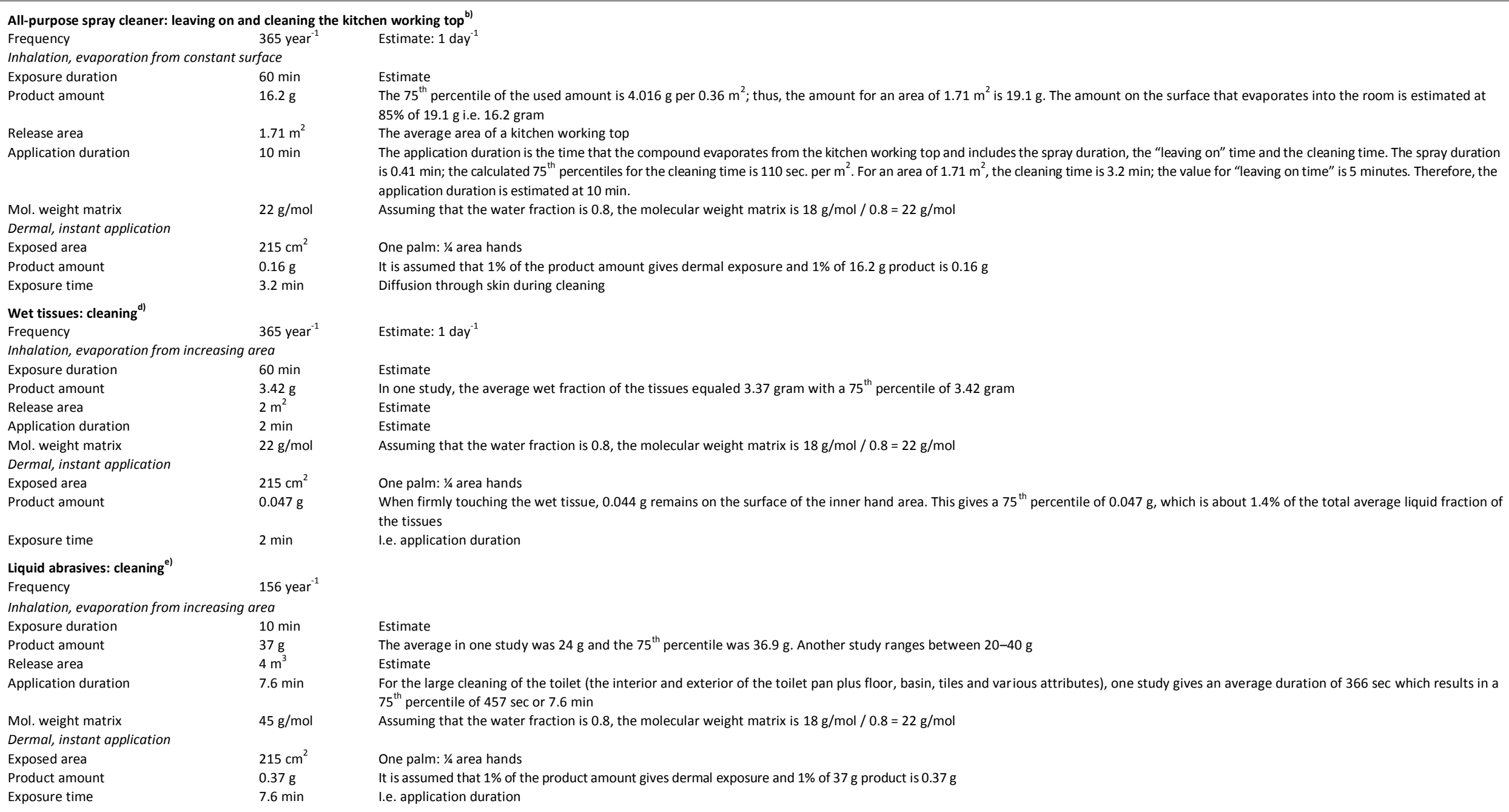




\section{Abrasive powders: scattering ${ }^{\text {b) }}$}

Frequency

104 year $^{-1}$

See "All-purpose cleaner liquid: mixing and loading"

Inhalation, spray model (inhalation/ oral exposure can occur to particles which whirl around in the air when using powders)

"Spray" duration $1 \mathrm{~min}$

Exposure duration

Mass generation rate

$60 \mathrm{~min}$

$0.62 \mathrm{~g} / \mathrm{sec}$

$0.2 \mathrm{~g} / \mathrm{g}$

$1 \mathrm{~g} / \mathrm{g}$

$1 \mathrm{~g} / \mathrm{g}$

Weight fraction non-volat

ensity non-volatile

$3 \mathrm{~g} / \mathrm{cm}^{3}$

Initial particle distribution Median (CV) 75 (0.6)

Inhalation cut-off diameter

$15 \mu \mathrm{m}$

Dermal, constant rate

$5.0 \mathrm{mg} / \mathrm{min}$

Release duration

$1 \mathrm{~min}$

Bathroom cleaning spray: spraying the shower walls

Erequency

Inhalation, spray model

Spray duration

Exposure duratio

Mass generation rate

$1.5 \mathrm{~min}$

$25 \mathrm{~min}$

$25 \mathrm{~min}$

Airborne fraction

Weight fraction non-volatile

Density non-volatile

Initial particle distribution

$0.2 \mathrm{~g} / \mathrm{g}$

$0.1 \mathrm{~g} / \mathrm{g}$

$1.8 \mathrm{~g} / \mathrm{cm}^{3}$

Median (CV)

Inhalation cut-off diamete

$100 \mu \mathrm{m}(0.6)$

$15 \mu \mathrm{m}$

Contact rate

$46 \mathrm{mg} / \mathrm{min}$

$1.5 \mathrm{~min}$

Bathroom cleaning spray: cleaning the shower walls $s^{\text {a }}$

Bathroom cleaning spray: cleaning the shower wall
Frequency 52 year $^{-1}$

Frequion, evaporation from

$\begin{array}{ll}\text { Exposure duration } & 25 \mathrm{~min} \\ \text { Product amount } & 30 \mathrm{~g}\end{array}$

Release area

Application duration

Mol. weight matrix

Dermal, instant application

Exposed area

Product amount

Exposure time, diffusion through skin $20 \mathrm{~m}$

Estimate

The default value

Product

95\% salts, see Table 11.2.2.1.

Based on a study. See Table 11.2.21.

See Table 11.2.2.1.

e. "spray" duration

Estimate $\mathrm{min}$, the default value mass generation rate is $0.39 \mathrm{~g} / \mathrm{sec}$.

See Table 11.2.2.1.

see Table 11.2.2.1.

Table 11.2.2.1.

See Table 11.2.2.1

ee Table 11.2.2.1.

l.e. spray duration

See "Bathroom cleaning spray: spraying the shower walls"

\section{Estimate}

based on different studies

$6.4 \mathrm{~m}^{2}$

$36 \mathrm{~g} / \mathrm{mol}$

One palm: $1 / 4$ area hands

I.e. application duration
For the scattering of powder on the kitchen working top and gas stove, the "spray" duration is estimated at 1 min.

In one study, the dermal exposure on hands and forearms ranges from 0.4 to $4.18 \mathrm{mg} / \mathrm{min}$ with a $75^{\text {th }}$ percentile of $2.83 \mathrm{mg} / \mathrm{min}$. The dermal exposure for legs, feet and face ranges from 0.22 to $6.56 \mathrm{mg} / \mathrm{min}$ with a $75^{\text {th }}$ percentile of $2.15 \mathrm{mg} / \mathrm{min}$. Using these data, the default value for contact rate is set at $5.0 \mathrm{mg} / \mathrm{minute}$

Based on two studies, the bathroom walls is cleaned once a week

The calculated $75^{\text {th }}$ percentile for the spraying is derived from one study $\left(14.3 \mathrm{sec}\right.$. per $\left.\mathrm{m}^{2}\right)$. For an area of $6.4 \mathrm{~m}^{2}$, the extrapolated spraying time is 1.5 minutes.

The default value for the mass generation rate of trigger sprays is set at $0.78 \mathrm{~g}$ product $/ \mathrm{sec}$. As it is assumed that active spraying occurred for a period of $0.75 \mathrm{~min}$ during a time span of 1.5

Dased on the general composition, it is assumed that the non-volatile part in bathroom cleaning sprays is about $10 \%$; the default value weight fraction non-volatile is set at $0.1 \mathrm{~g} / \mathrm{g}$.

On study gives an amount for surface spray from minimal 5 to maximal $30 \mathrm{gram}$. The average mass generation rate is set at $0.39 \mathrm{~g} / \mathrm{s}$ with a spray duration of 90 sec. Therefore, the amount for spraying is $0.39 \mathrm{~g} / \mathrm{s} \times 90 \mathrm{~s}=35.1 \mathrm{~g}$; the amount on the surface is estimated at $85 \%$ of $35.1 \mathrm{~g}$ and is $30 \mathrm{gram}$, which is set as default value.

The walls of a shower cubicle with an area of $0.80 \mathrm{~m}$ (width) $\times 2.0 \mathrm{~m}$ (height) $\times 4=6.4 \mathrm{~m}^{2}$

Assuming that the water fraction is 0.5 , the molecular weight matrix is $18 \mathrm{~g} / \mathrm{mol} / 0.5=36 \mathrm{~g} / \mathrm{mol}$

It is assumed that $1 \%$ of the product amount gives dermal exposure and $1 \%$ of $30 \mathrm{~g}$ product is 0.3 
Inhalation, evaporation from constant surface Exposure duration $\quad 0.75 \mathrm{~min}$

Product amount

Room volume

$500 \mathrm{~g}$

See "Laundry liquid: filling the washing machine"

See "Laundry liquid: filling the washing machine"

Release area

$\begin{array}{ll}1 \mathrm{~m}^{3} & \text { See "Laundry powder: filling the washing machine" } \\ 0.002 \mathrm{~m}^{2} & \text { See "Laundry powder: filling the washing machine" }\end{array}$

Application duration

Dermal, instant application

$0.3 \mathrm{~min}$

Assuming that the water fraction is 0.7 , the molecular weight matrix is $18 \mathrm{~g} / \mathrm{mol} / 0.7=26 \mathrm{~g} / \mathrm{mol}$

Exposed area

$215 \mathrm{~cm}^{2}$

Product amount

One palm: $1 / 4$ area hands

Exposure time $\quad 0.75 \mathrm{~min}$

See "Laundry powder: filling the washing machine"

Frequency $\quad 4$ year ${ }^{-1}$

Inhalation, evaporation from increasing area

Exposure duration $25 \mathrm{~min}$

Product amount dilution

Scaling): cleaning the shower walls ${ }^{\text {a) }}$

Estimate

Weight fraction dilution $\quad \mathrm{W}_{\mathrm{f}} / 45$

\section{Release area}

Application duration

The amount, which is used for cleaning, is estimated at $40 \mathrm{ml}$ of the diluted product per $\mathrm{m} 2$. For $6.4 \mathrm{~m} 2$, an amount of $260 \mathrm{ml}$ or 260 gram of the diluted product is required for cleaning. Only the amount on the walls of the shower cubicle is taken into account for the default.

Dilution $45 \mathrm{x}$. Per 2 litres wash water volume, $44 \mathrm{~g}$ bathroom cleaner is used, which gives a dilution of 45 . The weight of the suds is the weight fraction of the product divided by the dilution factor $\left(W_{f} / 45\right)$.

$6.4 \mathrm{~m}^{2} \quad$ See "Bathroom cleaning spray: cleaning the shower walls"

See "Bathroom cleaning sp say:

Mol. weight matrix

$20 \mathrm{~min}$

Dermal, instant application

$18 \mathrm{~g} / \mathrm{mol}$

Exposed area

Matrix is water

Weight fraction dilution $1900 \mathrm{~cm}^{2}$

Product amount dilution

Concentration dilution (diffusion

Area hands and forearm

$\begin{array}{ll}\mathrm{W}_{\mathrm{f}} / 45 & \text { Per } 2 \text { litres wash wate } \\ 19 \mathrm{~g} & \text { Skin layer is } 0.01 \mathrm{~cm}\end{array}$

through skin)

Skin layer is $0.01 \mathrm{~cm}$
Density water $1 \mathrm{~g} / \mathrm{cm}^{3}$

Exposure time

$\mathrm{W}_{\mathrm{f}} / 45 \mathrm{~g} / \mathrm{cm}^{3}$

Toilet cleaner, acid and bleach ${ }^{\mathrm{e}}$

Frequency

Acid clean

$20 \min$

l.e. application duration

260 year $^{-1}$ 120 year ${ }^{-1}$

Inhalation, evaporation from constant surface

Exposure duration $\quad 3 \mathrm{~min}$

Product amount dilution $1000 \mathrm{~g}$

Weight fraction dilution

Acid cleaner

Bleach

$w^{1 / 18}$

One study found a mean frequency of 0.3 day $^{-1}$ which equals 2.1 week $^{-1}$. The calculated $75^{\text {th }}$ percentile is 0.708 day $^{-1}$ or 5.0 week $^{-1}$

The same study found a mean frequency of 7 times a month with a $75^{\text {th }}$ percentile of 10 month $^{-1}$ or 120 year ${ }^{-1}$.

Estimate

Acid cleaner \& bleach. One study found an average amount of 40 gram undiluted toilet cleaner with a $75^{\text {th }}$ percentile of 55 gram. For cleaning the toilet with bleach the average amount was $55 \mathrm{~g}$ which gives a $75^{\text {th }}$ percentile of $80 \mathrm{gram}$. The total amount of toilet cleaner and water in the toilet pan is estimated at 1 litre

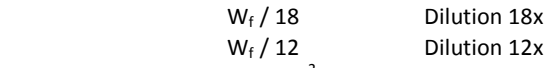

$0.075 \mathrm{~m}^{2}$

$2 \mathrm{~min}$

For the release area, it is assumed that evaporation takes place from the toilet pan. The area is estimated at $750 \mathrm{~cm}^{2}$.

In one study, the duration ranged from 10 to 150 seconds. For application duration, the default value is set at $2 \mathrm{~min}$.

Mol. weight matrix

$18 \mathrm{~g} / \mathrm{mol} \quad$ Matrix is water 
Default Value comments

\begin{tabular}{|c|c|c|}
\hline \multicolumn{3}{|l|}{ Dermal, instant application } \\
\hline Exposed area & $215 \mathrm{~cm}^{2}$ & One palm: $1 / 1$ area hands \\
\hline Product amount dilution & $2.2 \mathrm{~g}$ & Skin layer $0.01 \mathrm{~cm}$ \\
\hline \multicolumn{3}{|l|}{ Weight fraction dilution } \\
\hline Acid cleaner & $W_{f} / 18$ & Dilution $18 \mathrm{x}$ \\
\hline Bleach & $W_{f} / 12$ & Dilution $12 x$ \\
\hline \multicolumn{3}{|c|}{ Concentration dilution (diffusion through skin) } \\
\hline Acid cleaner & $W_{f} / 18$ & Density $1 \mathrm{~g} / \mathrm{cm}^{3}$ \\
\hline Bleach & $W_{f} / 12$ & Density $1 \mathrm{~g} / \mathrm{cm}^{3}$ \\
\hline Exposure time & $3 \mathrm{~min}$ & Based on two studies \\
\hline \multicolumn{3}{|l|}{ Toilet rim cleaner ${ }^{\mathrm{e})}$} \\
\hline \multicolumn{3}{|l|}{ Inhalation, constant rate } \\
\hline Exposure duration & $1440 \mathrm{~min}$ & I.e. 24 hours \\
\hline \multicolumn{3}{|l|}{ Product amount } \\
\hline Solid & $30 \mathrm{~g}$ & Product information \\
\hline Liquid & $70 \mathrm{~g}$ & For a liquid toilet rim cleaner, containing $55 \mathrm{ml}$ fluid (product information) with an estimated density of $1.3 \mathrm{~g} / \mathrm{cm}^{3}$, the amount is about $70 \mathrm{~g}$. \\
\hline \multicolumn{3}{|r|}{ 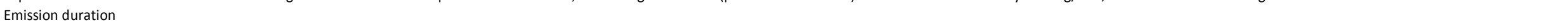 } \\
\hline Solid & $43200 \mathrm{~min}$ & Assuming that a toilet block of $30 \mathrm{gram}$ can last for 30 days (product information), the default value for emission duration is set at 43200 minutes. \\
\hline Liquid & $86400 \mathrm{~min}$ & As it is assumed that a liquid product can last for 60 days, the emission duration is set at 86400 minutes \\
\hline Inhalation rate & $9 \mathrm{~L} / \mathrm{min}$ & Rest \\
\hline \multicolumn{3}{|l|}{ Floor cleaning liquid: cleaning ${ }^{c)}$} \\
\hline Frequency & 104 year $^{-1}$ & Estimate \\
\hline \multicolumn{3}{|c|}{ Inhalation, evaporation from increasing area } \\
\hline Exposure duration & $240 \mathrm{~min}$ & Default \\
\hline Product amount dilution & $880 \mathrm{~g}$ & $\begin{array}{l}\text { When cleaning a surface, the amount of solution which is left on the cleaned surface is estimated at } 40 \mathrm{ml} \text { of the diluted product per } \mathrm{m} 2 \text {. For } 22 \mathrm{~m} 2 \text {, an amount of } 880 \mathrm{ml} \text { or } 880 \mathrm{gram} \text { is left } \\
\text { on the surface. }\end{array}$ \\
\hline Weight fraction dilution & $W_{f} / 20$ & $\begin{array}{l}\text { The concentration used in a solution of combined floor products ranges from } 0.5 \% \text { to } 2.5 \%) \text {. Floor cleaners are applied in a dilution of } 0.5 \% \text { to } 5.0 \%) \text {. The latter is taken as concentration for the diluted } \\
\text { product; for making a } 5 \text { litres cleaning solution, an amount of } 250 \text { gram is needed; this value is also given in the production information of linoleum cleaners. The dilution is then } 20 \text { times. }\end{array}$ \\
\hline Release area & $22 \mathrm{~m} 2$ & Living room \\
\hline Application duration & $30 \mathrm{~min}$ & Estimate \\
\hline Mol. weight matrix & $18 \mathrm{~g} / \mathrm{mol}$ & Matrix is water \\
\hline \multicolumn{3}{|l|}{ Dermal, instant application } \\
\hline Exposed area & $1900 \mathrm{~cm}^{2}$ & Area hands and forearms \\
\hline Weight fraction dilution & $W_{f} / 20$ & See "Inhalation" \\
\hline Product amount dilution & $19 \mathrm{~g}$ & Skin layer $0.01 \mathrm{~cm}$ \\
\hline $\begin{array}{l}\text { Concentration dilution (diffusion } \\
\text { through skin) }\end{array}$ & $W_{f} / 20 \mathrm{~g} / \mathrm{cm}^{3}$ & Density water $1 \mathrm{~g} / \mathrm{cm}^{3}$ \\
\hline Exposure time & $30 \mathrm{~min}$ & I.e. application duration \\
\hline \multicolumn{3}{|l|}{ Floor mopping systems ${ }^{c)}$} \\
\hline Frequency & 104 year $^{-1}$ & Estimate \\
\hline \multicolumn{3}{|c|}{ Inhalation, evaporation from increasing area } \\
\hline Exposure duration & $240 \mathrm{~min}$ & Default \\
\hline Product amount & $245 \mathrm{~g}$ & One study has found that for an area of $60 \times 60 \mathrm{~cm}$, the amount sprayed was $3.35 \mathrm{~g}$ with a calculated $75^{\text {th }}$ percentile of $4.016 \mathrm{~g}$. Thus, to clean an area of $22 \mathrm{~m} 2$ an amount of $245 \mathrm{~g}$ is needed. \\
\hline Release area & $22 \mathrm{~m}^{2}$ & Living room \\
\hline Application duration & $30 \mathrm{~min}$ & Estimate \\
\hline Mol. weight matrix & $22 \mathrm{~g} / \mathrm{mol}$ & The fraction of water in the product is estimated at 0.8 . For the molecular weight matrix, the default value is $18 \mathrm{~g} / \mathrm{mol} / 0.8=22 \mathrm{~g} / \mathrm{mol}$ \\
\hline
\end{tabular}




\begin{tabular}{|c|c|c|}
\hline \multicolumn{3}{|l|}{ Dermal, instant application } \\
\hline Exposed area & $215 \mathrm{~cm}^{2}$ & One palm: $1 / 4$ area hands \\
\hline Product amount & $0.25 \mathrm{~g}$ & $\begin{array}{l}\text { Dermal exposure occurs when the pad is taken of the mop head and afterwards. The exposure duration for dermal exposure is set at } 3 \mathrm{~min} \text {. It is assumed that } 10 \% \text { of the used amount remains in the pad } \\
\text { i.e. } 24.5 \mathrm{~g} \text { and that } 1 \% \text { of this amount contacts the skin resulting in a product amount of } 0.25 \text { gram. }\end{array}$ \\
\hline Exposure time & $3 \min$ & Estimate. See "Product amount - dermal" \\
\hline \multicolumn{3}{|c|}{ Floor polish (water based) $)^{c}$} \\
\hline Frequency & 2 year $^{-1}$ & Product information supported by two studies \\
\hline \multicolumn{3}{|c|}{ Inhalation, evaporation from increasing area } \\
\hline Exposure duration & $90 \mathrm{~min}$ & After polishing the floor, it is assumed that the user will leave the room (product information). \\
\hline Product amount & $550 \mathrm{~g}$ & $\begin{array}{l}\text { For wax or for floor polish parquet / linoleum, the product information gives that } 1 \text { litre product is sufficient for } 40 \mathrm{~m} 2 \text {. For polishing the floor area of the living room, the product amount is } \\
\text { estimated at } 550 \text { gram for } 22 \mathrm{~m} 2\end{array}$ \\
\hline Release area & $22 \mathrm{~m}^{2}$ & Living room \\
\hline Application duration & $90 \mathrm{~min}$ & For use duration, one study gave a $75^{\text {th }}$ percentile of 90 minutes which is set as default value. \\
\hline Mol. weight matrix & $22 \mathrm{~g} / \mathrm{mol}$ & $\begin{array}{l}\text { Assuming that the water fraction is } 0.8 \text {, the molecular weight matrix is } \\
18 \mathrm{~g} / \mathrm{mol} / 0.8=22 \mathrm{~g} / \mathrm{mol}\end{array}$ \\
\hline \multicolumn{3}{|l|}{ Dermal, instant application } \\
\hline Exposed area & $430 \mathrm{~cm} 2$ & One hand: $1 / 2$ area hands \\
\hline Product amount & $5.5 \mathrm{~g}$ & $1 \%$ of $550 \mathrm{~g}$ product \\
\hline Exposure time & $90 \mathrm{~min}$ & See "Exposure duration - inhalation" \\
\hline \multicolumn{3}{|c|}{ Carpet cleaning liquid: mixing and loading } \\
\hline Frequency & 0.5 year $^{-1}$ & Based on one study and the product information \\
\hline \multicolumn{3}{|c|}{ Inhalation, evaporation from constant surface } \\
\hline Exposure duration & $0.75 \mathrm{~min}$ & $\begin{array}{l}\text { For mixing and loading } 60 \mathrm{~g} \text { all-purpose cleaner in } 5 \text { litres water, an exposure duration of } 0.75 \text { minutes is given under "all-purpose cleaner liquid: mixing and loading". For the mixing and loading of } \\
500 \mathrm{ml} \text { carpet cleaner in } 10 \text { litres water, the exposure duration is also set } 0.75 \text { minutes. }\end{array}$ \\
\hline Product amount & $500 \mathrm{~g}$ & See "Laundry liquid: filling the washing machine" \\
\hline Room volume & $1 \mathrm{~m}^{3}$ & See "Laundry liquid: filling the washing machine" \\
\hline Release area & $0.002 \mathrm{~m}^{2}$ & See "Laundry liquid: filling the washing machine" \\
\hline Application duration & $0.3 \mathrm{~min}$ & For mixing and loading $60 \mathrm{~g}$ all-purpose cleaner in 5 litres water, application duration of 0.3 minutes is given in "all-purpose cleaner liquid: mixing and loading" \\
\hline Mol. weight matrix & $36 \mathrm{~g} / \mathrm{mol}$ & Assuming the product contains $50 \%$ water, the molecular weight matrix is $18 \mathrm{~g} / \mathrm{mol} / 0.5=36 \mathrm{~g} / \mathrm{mol}$ \\
\hline \multicolumn{3}{|l|}{ Dermal, instant application } \\
\hline Exposed area & $215 \mathrm{~cm} 2$ & One palm: $1 / 4$ area hands \\
\hline Product amount & $0.010 \mathrm{~g}$ & See "Laundry liquid: filling the washing machine" \\
\hline Exposure time & $0.75 \mathrm{~min}$ & I.e. exposure duration \\
\hline \multicolumn{3}{|c|}{ Carpet cleaner liquid: manual cleaning of fitted carpet $\left.{ }^{c}\right)$} \\
\hline Frequency & 0.5 year $^{-1}$ & See "Carpet cleaning liquid: mixing and loading" \\
\hline \multicolumn{3}{|c|}{ Inhalation, evaporation from increasing area } \\
\hline Exposure duration & $110 \mathrm{~min}$ & $\begin{array}{l}\text { When the whole area of the carpet is cleaned, the user leaves the room. So, the exposure duration is the same as the application duration. For a thorough cleaning of carpet the use dura- } \\
\text { tion and exposure duration are estimated at } 5 \text { minutes per } \mathrm{m} 2 \text {. Thus, for } 22 \mathrm{~m} 2 \text { carpet the cleaning duration is } 110 \text { minutes. }\end{array}$ \\
\hline Product amount dilution & $10000 \mathrm{~g}$ & $\begin{array}{l}\text { The amount for carpet and upholstery cleaner is given as } 500 \mathrm{ml} \text { for } 15-20 \mathrm{~m} 2 \text { (product information). This quantity should be diluted to } 10 \text { litres; therefore, the maximal amount of the } \\
\text { diluted cleaner is } 10000 \mathrm{ml} / 22 \mathrm{~m} 2=450 \mathrm{ml} / \mathrm{m} 2 \text {. Foam cleaning and spray extraction leave up to } 0.5 \mathrm{I} \text { cleaning solution } / \mathrm{m} 2 \text { carpet behind, requiring a drying time of up to } 4 \text { days. For } \\
\text { (diluted) product amount the default value is set at } 2200 \mathrm{ml} \text {. }\end{array}$ \\
\hline Weight fraction dilution & $W_{f} / 20$ & The weight fraction must be divided by the dilution factor, which is 20 times ( $500 \mathrm{ml}$ in 10 litres). \\
\hline Release area & $22 \mathrm{~m}^{2}$ & Living room \\
\hline Application duration & $110 \mathrm{~min}$ & See "Exposure duration" \\
\hline Mol. weight matrix & $18 \mathrm{~g} / \mathrm{mol}$ & Matrix is water \\
\hline
\end{tabular}




\begin{tabular}{|c|c|c|}
\hline \multicolumn{3}{|l|}{ Dermal, instant application } \\
\hline Exposed area & $860 \mathrm{~cm} 2$ & Area hands \\
\hline Weight fraction dilution & $W_{f} / 20$ & See "Weight fraction dilution - inhalation" \\
\hline Product amount dilution & $27 \mathrm{~g}$ & $\begin{array}{l}\text { it is assumed that } 0.27 \% \text { of the applied stain ends up on the skin. For cleaning } 22 \mathrm{~m} 2 \text { carpet } 10 \text { litres solution is needed; } 0.27 \% \text { equals } 27 \mathrm{ml} \text { diluted carpet cleaner. The default value for } \\
\text { product amount is set at } 27 \mathrm{~g} \text {. }\end{array}$ \\
\hline Concentration - diffusion through skin & $W_{f} / 20$ & Density $1 \mathrm{~g} / \mathrm{cm}^{3}$ \\
\hline Exposure time & $110 \mathrm{~min}$ & See "Exposure duration - inhalation" \\
\hline \multicolumn{3}{|l|}{ (arpet powder: scattering ${ }^{c)}$} \\
\hline Frequency & 0.5 year $^{-1}$ & See "Carpet cleaning liquid: mixing and loading" \\
\hline \multicolumn{3}{|l|}{ Inhalation, spray model } \\
\hline Spray duration & $22 \mathrm{~min}$ & Estimate \\
\hline Exposure duration & $22 \mathrm{~min}$ & Estimate \\
\hline Mass generation rate & $1.7 \mathrm{~g} / \mathrm{sec}$ & $\begin{array}{l}\text { For cleaning the carpet, an amount of } 50-100 \mathrm{~g} \text { per } \mathrm{m} 2 \text { is given (product information). It is assumed that } 2200 \mathrm{gram} \text { is necessary for dusting the fitted carpet of the living room. If } 100 \mathrm{~g} \text { is } \\
\text { scattered in one minute, } 2200 \mathrm{~g} \text { of powder is scattered in } 22 \mathrm{~min} \text {. and the mass generation rate is } 100 \mathrm{~g} / \mathrm{min}=1.7 \mathrm{~g} / \mathrm{sec} \text {. }\end{array}$ \\
\hline Airborne fraction & 0.2 & No available data. See Table 11.2.2.1. "Trigger sprays" \\
\hline Weight fraction non-volatile & $1 \mathrm{~g} / \mathrm{g}$ & Product \\
\hline Density non-volatile & $1.8 \mathrm{~g} / \mathrm{cm}^{3}$ & See Table 11.2.2.1. "Trigger sprays" \\
\hline Initial particle distribution & $75(0.6)$ & Based on one study \\
\hline \multicolumn{3}{|l|}{ Median (CV) } \\
\hline \multirow{2}{*}{\multicolumn{3}{|c|}{ Dermal, constant rate }} \\
\hline & & \\
\hline Contact rate & $5.0 \mathrm{mg} / \mathrm{min}$ & $\begin{array}{l}\text { In one study, the dermal exposure on hands and forearms ranges from } 0.4 \text { to } 4.18 \mathrm{mg} / \mathrm{min} \text { with a } 75^{\text {th }} \text { percentile of } 2.83 \mathrm{mg} / \mathrm{min} \text {. The dermal exposure for legs, feet and face ranges from } \\
0.22 \text { to } 6.56 \mathrm{mg} / \mathrm{min} \text { with a } 75^{\text {th }} \text { percentile of } 2.15 \mathrm{mg} / \mathrm{min} \text {. Using these data, the default value for contact rate is set at } 5.0 \mathrm{mg} / \mathrm{minute} \text {. }\end{array}$ \\
\hline Release duration & $22 \mathrm{~min}$ & I.e. "spray" duration \\
\hline Uptake fraction & 1 & Potential dose \\
\hline \multicolumn{3}{|l|}{ Carpet powder: post- application } \\
\hline Frequency & 14 year $^{-1}$ & It is assumed that a child (default 10.5 months) crawls over the cleaned surface for 1 hour a day during a 14 -day period \\
\hline Body weight & $8.69 \mathrm{~kg}$ & Child 10.5 months \\
\hline \multicolumn{3}{|l|}{ Dermal, rubbing off } \\
\hline Transfer coefficient & $0.6 \mathrm{~m}^{2} / \mathrm{hr}$ & $\begin{array}{l}\text { The "transfer coefficient" is a quantification of the transfer of powder from the carpet surface to human skin per unit time as e.g. a child is crawling across a treated carpet, wiping some of } \\
\text { the powder. For infants aged } 6 \text { months to } 1-1.5 \text { years, an assumed mean dermal transfer coefficient of } 0.6 \mathrm{~m} 2 / \mathrm{hr} \text { is given }\end{array}$ \\
\hline Dislodgeable amount & $3 \mathrm{~g} / \mathrm{m}^{2}$ & $\begin{array}{l}\text { For transfer efficiency for dried fluid, studies range between } 3 \% \text { (painted wood, MDF) to } 60 \% \text { (brown rough glazed tile). Based on these studies, the default value for dislodgeable fraction is } \\
\text { set at } 30 \% \text {. It is assumed that after vacuuming } 10 \% \text { of the used amount settles on the carpet from which a certain fraction is dislodgeable. If } 2200 \mathrm{~g} \text { of carpet powder is scatter ed onto } 22 \\
\mathrm{~m} 2 \text {, the dislodgeable product amount will be } 100 \mathrm{~g} / \mathrm{m} 2 \times 0.1 \text { (residual fraction) } \times 0.3 \text { (dislodgeable fraction) }=3 \mathrm{~g} \text { product } / \mathrm{m} 2 \text {. Thus, the dislodgeable amount of carpet powder is } 3 \mathrm{~g} / \mathrm{m} 2 \text {. }\end{array}$ \\
\hline Contact time & $60 \mathrm{~min}$ & It is assumed that a child (default 10.5 months) crawls over the cleaned surface for 1 hour a day during a 14-day period \\
\hline Rubbed surface & $22 \mathrm{~m}^{2}$ & Living room \\
\hline Exposed area & $1170 \mathrm{~cm}^{2}$ & $\begin{array}{l}\text { Infant surface area exposed is calculated based on a child wearing a short-sleeved shirt and a napkin, and no socks or shoes. The exposed area considered is, therefore, hands, feet, fore- } \\
\text { arms and lower legs. The body part percentages of total surface area of exposed body surface area are for hands and feet } 5.3 \% \text { and } 7.1 \% \text {, respectively, for forearms and lower legs the } \\
\text { percentages are } 6.2 \% \text { and } 8.2 \% \text {, respectively. The total exposed body part percentage is } 26.8 \% \text {, the total surface area body is } 4370 \mathrm{~cm}^{2} \text {, and as a result, the exposed area is } 1170 \mathrm{~cm}^{2} \text {. }\end{array}$ \\
\hline \multicolumn{3}{|r|}{ 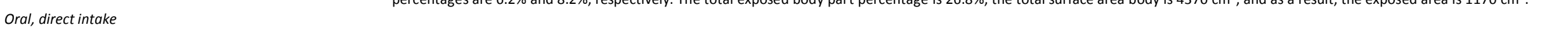 } \\
\hline Amount ingested & $\begin{array}{l}10 \% \text { of the } \\
\text { external dermal } \\
\text { exposure }\end{array}$ & $\begin{array}{l}\text { It is assumed that } 10 \% \text { of the used amount settles on the carpet. It is assumed that } 50 \% \text { of the product that ends up on the hands is taken in orally. This means that via hand-mouth contact } 10 \% \text { of } \\
\text { the calculated external dermal exposure is ingested and that the internal dermal exposure is } 90 \% \text { of the calculated external dermal exposure. }\end{array}$ \\
\hline \multicolumn{3}{|l|}{ Carpet spot removers } \\
\hline Frequency & 10 year $^{-1}$ & Based on one study where the $75^{\text {th }}$ percentile of frequency was 10 times a year \\
\hline \multicolumn{3}{|l|}{ Dermal, instant application } \\
\hline Exposed area & $215 \mathrm{~cm}^{2}$ & One palm: $1 / 4$ area hands \\
\hline Product amount & $0.07 \mathrm{~g}$ & The default value for amount is set at $7 \mathrm{~g}$ (based on product information). Worst-case is estimated that $1 \%$ of the product amount contacts the skin; i.e. $0.07 \mathrm{~g}$ \\
\hline Exposure time & $10 \mathrm{~min}$ & Estimate \\
\hline
\end{tabular}


Furniture polish: manually rubbing a cupboard ${ }^{c)}$

Frequency

1 year $^{-1}$

Inhalation, evaporation from increasing area

Exposure duration $240 \mathrm{~min}$

Product amount

$550 \mathrm{~g}$

Release area

Application duration
Mol. weight matrix

$22 \mathrm{~m}^{2}$

$90 \mathrm{~min}$

$272 \mathrm{~g} / \mathrm{mol}$

Dermal, instant application

Exposed area

Product amount

$430 \mathrm{~cm}^{2}$

$430 \mathrm{~cm}^{2}$
$5.5 \mathrm{~g}$

$90 \mathrm{~min}$

Leather furniture spray: spraying a leather sofac)

Frequency

1 year $^{-1}$

Exposure duration

Mass generation

Wirborne fraction

Weight fraction non-
Density non-volatile

Density non-volatile
Initial particle distribution

Initial particle

Median (CV)
Inhalation cut-off diameter

Inhalation cut-off diame

Contact rate

Release duration

Glass cleaner: spraying ${ }^{c)}$

Frequency

Inhalation, spray model

Spray duration

Exposure duration

Mass generation rate

Airborne fraction

Weight fraction non-volatile

Density non-volatile

Initial particle distribution

Median (CV)

Inhalation cut-off diamete

Dermal, constant rate

Contact rate

Release duration
Estimate

Estimate

For wax or for floor polish parquet, the product information gives that 1 litre product is sufficient for $40 \mathrm{~m} 2$. Treating parquet or a cupboard is comparable; therefore, for polishing the cupboard surface, the product amount is estimated at 550 gram for $22 \mathrm{~m} 2$.

The cupboard measures $1.2 \mathrm{~m}$ (width) $\times 1.9 \mathrm{~m}$ (height) $\times 0.6 \mathrm{~m}$ (depth) and it has 4 shelves. Thus, an area of $22 \mathrm{~m} 2$ is treated with undiluted furniture polish

Based on the $75^{\text {th }}$ percentile in one study

For products containing oil or wax, the density is about $0.8 \mathrm{~g} / \mathrm{cm}^{3}$. Assuming that turpentine is a solvent in these products, fraction 0.5 and $\mathrm{Mw} 136$, the molecular weight matrix is calculated as follows: $136 / 0.5=272 \mathrm{~g} / \mathrm{mol}$

One hand: $1 / 2$ area hands

$1 \%$ of $550 \mathrm{~g}$ product

e. application duration

Estimate

$3 \min \quad$ Estimate

$0.75 \mathrm{~g} / \mathrm{sec} \quad$ See Table 11.2.2.1.

$1 \mathrm{~g} / \mathrm{g} \quad$ See Table 11.2.2.1.

$1 \mathrm{~g} / \mathrm{g}$ S/g $\quad$ See Table 11.2.2.1.

$1.8 \mathrm{~g} / \mathrm{cm}^{3} \quad$ See Table 11.2.2.1

$25 \mu \mathrm{m}(0.4) \quad$ See Table 11.2.2.1.

$15 \mu \mathrm{m} \quad$ See Table 11.2.2.1.

$100 \mathrm{mg} / \mathrm{min} \quad$ See Table 11.2.2.1

$3 \mathrm{~min} \quad$ I.e. spray duration

365 year $^{-1} \quad$ Estimate, 1 day $^{-1}$

$0.7 \mathrm{~min} \quad$ For spraying the default value is $14.3 \mathrm{sec} / \mathrm{m} 2$. Thus, for an area of $3 \mathrm{~m} 2$ the spraying time is $43 \mathrm{sec}$ or $0.7 \mathrm{~min}$.

$240 \mathrm{~min} \quad$ Estimate

$0.78 \mathrm{~g} / \mathrm{sec} \quad$ See Table 11.2.2.1

$0.2 \mathrm{~g} / \mathrm{g} \quad$ See Table 11.2.2.1.

Based on the general composition, it is assumed that the non-volatile part in glass cleaning sprays is about $5 \%$; the default value weight fraction non-volatile is set at $0.05 \mathrm{~g} / \mathrm{g}$

$1.8 \mathrm{~g} / \mathrm{cm} \quad$ See Table 11.2.2.1

$15 \mu \mathrm{m}$

See Table 11.2.2.1

$46 \mathrm{mg} / \mathrm{min} \quad$ See Table 11.2.2.1

$0.7 \mathrm{~min} \quad$ I.e. spray duration 


\begin{tabular}{|c|c|c|}
\hline \multicolumn{3}{|l|}{ Glass cleaner: cleaning } \\
\hline Frequency & 365 year $^{-1}$ & Estimate, day ${ }^{-1}$ \\
\hline \multicolumn{3}{|l|}{ Dermal, instant application } \\
\hline Exposed area & $215 \mathrm{~cm}^{2}$ & One palm: $1 / 4$ area hands \\
\hline Product amount & $0.29 \mathrm{~g}$ & $\begin{array}{l}\text { The sprayed amount is the mass generation rate multiplied with the spraying time i.e. } 0.78 \mathrm{~g} / \mathrm{s} \times 43 \mathrm{~s}=33.5 \mathrm{~g} \text {. The amount on the surface is estimated at } 85 \% \text { of } 33.5 \mathrm{~g} \text { i.e. } 28.5 \mathrm{gram} \text {. Worst- } \\
\text { case is estimated that } 1 \% \text { of the product amount contacts the skin i.e. } 0.29 \mathrm{~g} \text {. }\end{array}$ \\
\hline Exposure time & $6 \mathrm{~min}$ & For cleaning the default value is $110 \mathrm{sec} / \mathrm{m} 2$. Thus, for an area of $3 \mathrm{~m} 2$ the cleaning time is $330 \mathrm{sec}$ or $5.5 \mathrm{~min}$. The default for exposure time is set at 6 minutes. \\
\hline \multicolumn{3}{|c|}{ Oven cleaner, trigger spray: spraying ${ }^{b)}$} \\
\hline $\begin{array}{l}\text { Frequency } \\
\text { Inhalation, spray model }\end{array}$ & 26 year $^{-1}$ & Based on three studies $-75^{\text {th }}$ percentiles \\
\hline Spray duration & $0.5 \mathrm{~min}$ & $\begin{array}{l}\text { One study found for an area of } 60 \times 60 \mathrm{~cm} \text { a used amount of } 3.35 \mathrm{~g} \text { with a calculated } 77^{\text {th }} \text { percentile of } 4.016 \mathrm{~g} \text { and a maximum amount of } 4.8 \mathrm{~g} \text {. As default value for cleaning the oven twice } \\
\text { the maximum amount is taken i.e. } 26.7 \mathrm{~g} / \mathrm{m} 2 \text {; so, for an area of } 0.9 \mathrm{~m} 2 \text { an amount of } 24 \mathrm{~g} \text { is needed. The active spraying time is amount used divided by mass generation rate i.e. } 24 \mathrm{~g} / 0.78 \\
\mathrm{~g} / \mathrm{s}=30.8 \mathrm{sec}=0.5 \mathrm{~min} \text { which is s as default value for spray duration. }\end{array}$ \\
\hline Exposure duration & $60 \mathrm{~min}$ & Estimate \\
\hline Mass generation rate & $0.78 \mathrm{~g} / \mathrm{sec}$ & See Table 11.2.2.1. \\
\hline Airborne fraction & $0.2 \mathrm{~g} / \mathrm{g}$ & See Table 11.2.2.1. \\
\hline Weight fraction non-volatile & $0.1 \mathrm{~g} / \mathrm{g}$ & $\begin{array}{l}\text { It is assumed that oven cleaning trigger sprays have more or less the same composition as oven cleaning liquids which contain surfactants, build ers and bases ( } 0-15 \% \text {, see above). It is } \\
\text { assumed that the non-volatile part in oven cleaners is about } 10 \% \text {; the default value weight fraction non-volatile is set at } 0.1 \mathrm{~g} / \mathrm{g} \text {. }\end{array}$ \\
\hline Density non-volatile & $1.8 \mathrm{~g} / \mathrm{cm}^{3}$ & See Table 11.2.2.1. \\
\hline \multirow{2}{*}{\multicolumn{3}{|c|}{ Median (CV) }} \\
\hline & & \\
\hline Inhalation cut-off diameter & $15 \mu \mathrm{m}$ & See Table 11.2.2.1. \\
\hline \multicolumn{3}{|l|}{ Dermal, constant rate } \\
\hline Contact rate & $46 \mathrm{mg} / \mathrm{min}$ & See Table 11.2.2.1. \\
\hline Release duration & $0.5 \mathrm{~min}$ & I.e. spray duration \\
\hline \multicolumn{3}{|c|}{ Oven cleaner, trigger spray: cleaning } \\
\hline Frequency & 26 year $^{-1}$ & See "Oven cleaner, trigger spray: spraying" \\
\hline \multicolumn{3}{|l|}{ Dermal, instant application } \\
\hline Exposed area & $430 \mathrm{~cm}^{2}$ & One hand: $1 / 2$ area hands \\
\hline Product amount & $0.20 \mathrm{~g}$ & The sprayed amount is $24 \mathrm{~g}$. The amount on the surface is estimated at $85 \%$ of $24 \mathrm{~g}$ i.e. $20.4 \mathrm{gram}$. Worst-case is estimated that $1 \%$ of the product amount contacts the skin i.e. $0.2 \mathrm{~g}$. \\
\hline Exposure time & $20 \mathrm{~min}$ & Maximum use duration \\
\hline \multicolumn{3}{|l|}{ Metal cleaners: cleaning ${ }^{b)}$} \\
\hline Frequency & 6 year $^{-1}$ & Estimate, every two months \\
\hline \multicolumn{3}{|c|}{ Inhalation, evaporation from increasing area } \\
\hline Exposure duration & $60 \mathrm{~min}$ & Estimate \\
\hline Product amount & $10 \mathrm{~g}$ & Estimate \\
\hline Release area & $1.71 \mathrm{~m}^{2}$ & Estimate \\
\hline Application duration & $10 \mathrm{~min}$ & Estimate \\
\hline Mol. weight matrix & $22 \mathrm{~g} / \mathrm{mol}$ & Assuming that the water fraction is 0.8 , the molecular weight matrix is $18 \mathrm{~g} / \mathrm{mol} / 0.8=22 \mathrm{~g} / \mathrm{mol}$ \\
\hline \multicolumn{3}{|l|}{ Dermal, instant application } \\
\hline Exposed area & $215 \mathrm{~cm}^{2}$ & One palm: $1 / 4$ area hands \\
\hline Product amount & $0.10 \mathrm{~g}$ & $1 \%$ of $10 \mathrm{~g}$ product \\
\hline Exposure time & $10 \mathrm{~min}$ & I.e. application duration \\
\hline
\end{tabular}




\section{Shoe polish spray ${ }^{\natural)}$}

Frequency

Inhalation, spray model

Spray duration

Exposure duration

Mass generation rate

Airborne fraction

Weight fraction non-volatile

Density non-volatile

Initial particle distribution

Median (CV)

Inhalation cut-off diameter

Dermal, constant rate

Contact rate

Release duration

Shoe cream

Dermal, instant application

Exposed area

Product amount

Product amount

Exposure time

a) Bathroom - see Table 11.2.2.2.

b) Kitchen - see Table 11.2.2.2.

c) Living room - see Table 11.2.2.2.

d) Non-specified room - see Table 11.2.2.2.

e) Toilet - see Table 11.2.2.2.

f) Garage - see Table 11.2.2.2.

Source: Modified from the Cleaning Product Fact Sheet, RIVM (2006c). Estimate

$15 \mu \mathrm{m}$

$20 \mathrm{~min} \quad$ Estimate
Based on the $75^{\text {th }}$ percentile from one study

$1.2 \mathrm{~min} \quad$ One study gives a $75^{\text {th }}$ percentile of $284 \mathrm{~g}$ for used amount per year i.e. a used amount of $36 \mathrm{gram}$ per event. With a mass generation rate of $0.5 \mathrm{~g} / \mathrm{s}$, the calculated spray duration is $72 \mathrm{sec}$ or $1.2 \mathrm{~min}$.

The default value for the mass generation rate of sprays is $1.5 \mathrm{~g} / \mathrm{s}$. It is assumed that the use duration, the time during which the spraying takes place, is three times as long as the actua spraying time. The mass generation rate is set at $0.5 \mathrm{~g} / \mathrm{s}$

See Table 11.2.2.1.

Based on the general composition, it is assumed that the non-volatile part in shoe polish sprays is about $5 \%$; the default value weight fraction non-volatile is set at $0.05 \mathrm{~g} / \mathrm{g}$.

See Table 11.2.2.1.

See Table 11.2 .2 .1$.

See Table 11.2.2.1.

See Table 11.2.2.1

.e. spray duration

The frequency is estimated at once a fortnight i.e. 26 times a year. It is assumed that one uses shoe polish cream more frequently than shoe polish spray

$215 \mathrm{~cm}^{2} \quad$ One palm: $1 / 4$ area hands

$0.10 \mathrm{~g} \quad 1 \%$ of $10 \mathrm{~g}$ product 


\subsubsection{Do-it-yourself products}

In the Do-it-yourself Products Fact Sheet, RIVM (2007a), do-it-yourself (DIY) products are classified into 26 categories, which are characterized according to the type of use and exposure. DIY tasks are carried out mainly during leisure time. According to data from Statistics Netherlands (2005), approximately $25 \%$ of the Dutch population (age over 14 years) spends one to four hours per week on DIY tasks. Furthermore, Statistics Netherlands specifies that $6 \%$ of that population spends over five hours per week on DIY tasks during their leisure time (RIVM, 2007a).

Like in the two previous described Fact Sheets, default models and default parameter values are proposed for each product category. The default values provided in the Do-it-yourself Products Fact Sheet, RIVM (2007a), are mainly based on an observational study by RIVM by Magré from 2005 (Magré, 2005). However, the observations in this study were quite low. Therefore, the information gathered in the study can be used as preliminary data which can be considered indicative for consumer use of DIY products.

The exposure models are also thoroughly described in this Fact Sheet and will only shortly be mentioned under each product. The more complicated model "spraying" is already described in the two previous sections. A few general default values will be described here:

- Non-specified room: Unless it is obvious or specified where the task will be performed, a "non-specified room" will be considered. The non-specified room has a volume of $20 \mathrm{~m}^{3}$ with a ventilation rate of $0.6 \mathrm{~h}^{-1}$. The temperature is usually that of the room, which is set at $20^{\circ} \mathrm{C}$ and the standard room height is $2.5 \mathrm{~m}$ (RIVM, 2007a)

- The user: Users of DIY products are both men and women, (although men use these items more often. The average size of body parts for adults is used here from General Fact Sheet (RIVM, 2006a). The body weight of the adult user is set at $65 \mathrm{~kg}$ as a default. It is most likely that individuals will be dermally exposed to their hands while working with DIY products, where both intentionally or accidental contact may occur. The surface area for both hands is set at $860 \mathrm{~cm}^{2}$ by default, whereas sometimes only one hand or one palm will be taken as exposure area. These surface areas relate to the total surface area by factor 0.5 or 0.25 , respectively. Fingertips are quite often used to smooth joints made from sealants. Their surface area is set at $1 \mathrm{~cm}^{2}$ per fingertip (RIVM, 2007a)

- Molecular Weight Matrix: DIY products are in most cases mixtures of compounds; in the case of a mixture the evaporation is not only determined by the compound of interest, but also by the other constituents. The molecular weight of the matrix (or: mol. weight matrix) is the weighted average of the matrix, which contains the chemical of interest. The mol. weight matrix parameter is used to correct for the evaporation rate since it describes the average mol. 
weight of the rest of the total product (the product minus the compound of interest). This parameter can be left blank when the product purity is $100 \%$. The exact composition is not known in most cases. When the composition is unknown the mol. weight matrix will be set at $3000 \mathrm{~g} / \mathrm{mol}$ as a worst case value when nothing else is stated. Roughly, inserting this value in the evaporation model will result in an estimation of a product in its pure form (RIVM, 2007a)

Table 11.7 presents default values of DIY products from the Do-ityourself Products Fact Sheet, RIVM (2007a).

Table 11.7: Default Values on Do-it-yourself Products

\begin{tabular}{|c|c|c|}
\hline & Default Values & Comments \\
\hline \multicolumn{3}{|l|}{ Tube glue } \\
\hline Frequency & 1 week $^{-1}$ & Estimate \\
\hline \multicolumn{3}{|c|}{ Inhalation, evaporation from increasing area } \\
\hline Exposure duration & $240 \mathrm{~min}$ & Assuming that a person stays in the room after use \\
\hline Application duration & $10 \mathrm{~min}$ & Estimate \\
\hline Product amount & $9 \mathrm{~g}$ & Estimate \\
\hline Release area & $200 \mathrm{~cm}^{2}$ & $\begin{array}{l}\text { According to the directions for use of the products per } \\
100 \mathrm{ml} \text {, a surface of } 0.2 \mathrm{~m}^{2} \text { can be treated. The amount } \\
\text { of glue, set as default at } 10 \mathrm{ml} \text { per } 0.02 \mathrm{~m}^{2} \text {, will, for this } \\
\text { situation, be less, a product amount of } 9 \mathrm{~g} \text { is calculated } \\
\text { and a product density is } 0.9 \mathrm{~g} / \mathrm{cm}^{3} \text {. }\end{array}$ \\
\hline \multicolumn{3}{|c|}{ Dermal, instant application } \\
\hline Surface area & $2 \mathrm{~cm}^{2}$ & Two fingertips \\
\hline Product amount & $0.08 \mathrm{~g}$ & Estimate based on one study \\
\hline \multicolumn{3}{|c|}{ Bottled glue: universal/wood glue. } \\
\hline Frequency & 1 week $^{-1}$ & Hobby use \\
\hline \multicolumn{3}{|c|}{ Inhalation, evaporation from increasing area } \\
\hline Exposure duration & $240 \mathrm{~min}$ & $\begin{array}{l}\text { It is expected that the individual will stay in the room } \\
\text { for four hours in total. }\end{array}$ \\
\hline Application duration & $20 \mathrm{~min}$ & Estimate \\
\hline Product amount & $10 \mathrm{~g}$ & Estimate \\
\hline Release area & $400 \mathrm{~cm}^{2}$ & $\begin{array}{l}\text { For the hobby task it was estimated that approximately } 10 \\
\text { gram is needed (personal estimation). This relates to a } \\
\text { surface area of } 0.04 \mathrm{~m} 2 \text { according to product information. }\end{array}$ \\
\hline \multicolumn{3}{|c|}{ Dermal, instant application } \\
\hline Surface area & $2 \mathrm{~cm}^{2}$ & Two fingertips \\
\hline Product amount & $0.08 \mathrm{~g}$ & Estimate based on one study \\
\hline \multicolumn{3}{|c|}{ Bottled glue: construction glue } \\
\hline Frequency & 2 year $^{-1}$ & Estimate \\
\hline \multicolumn{3}{|c|}{ Inhalation, evaporation from increasing area } \\
\hline Exposure duration & $240 \mathrm{~min}$ & $\begin{array}{l}\text { Assembling a closet takes four hours (=exposure } \\
\text { duration) of which } 30 \text { minutes actual gluing. }\end{array}$ \\
\hline Application duration & $30 \mathrm{~min}$ & See "exposure duration" \\
\hline Product amount & $250 \mathrm{~g}$ & Product information - to treat an area of $1 \mathrm{~m}^{2}$ \\
\hline Release area & $1 \mathrm{~m}^{2}$ & Estimate \\
\hline \multicolumn{3}{|c|}{ Dermal, instant application } \\
\hline Surface area & $215 \mathrm{~cm}^{2}$ & Surface of one hand palm \\
\hline Product amount & $0.25 \mathrm{~g}$ & Estimate \\
\hline \multicolumn{3}{|l|}{ Super glue } \\
\hline Frequency & 1 month $^{-1}$ & Estimate \\
\hline \multicolumn{3}{|c|}{ Inhalation, evaporation from constant area } \\
\hline Exposure duration & $240 \mathrm{~min}$ & The subject stays in the room after use \\
\hline Application duration & $5 \mathrm{~min}$ & Estimate \\
\hline Product amount & $0.5 \mathrm{~g}$ & Based on one study \\
\hline Release area & $2 \mathrm{~cm}^{2}$ & Estimate based on product information \\
\hline \multicolumn{3}{|c|}{ Dermal, instant application } \\
\hline Surface area & $2 \mathrm{~cm}^{2}$ & Two fingertips \\
\hline Product amount & $25 \mathrm{mg}$ & Half a droplet size is considered $(25 \mathrm{mg}$ ) as default \\
\hline
\end{tabular}




\begin{tabular}{|c|c|c|}
\hline & Default Values & Comments \\
\hline \multicolumn{3}{|c|}{ Two-component glue: mixing and loading } \\
\hline Frequency & 3 year $^{-1}$ & Estimate \\
\hline \multicolumn{3}{|c|}{ Inhalation, evaporation from constant release area } \\
\hline Exposure duration & $5 \min$ & Estimate \\
\hline Application duration & $5 \mathrm{~min}$ & Estimate \\
\hline Product amount & $20 \mathrm{~g}$ & Product information: gluing a large broken vase \\
\hline Room volume & $1 \mathrm{~m}^{3}$ & $\begin{array}{l}\text { "Room volume" is interpreted here as personal space: a } \\
\text { small area of } 1 \mathrm{~m}^{3} \text { around the user. A small area around } \\
\text { the user is relevant for the inhalation exposure of the } \\
\text { user for the short use duration in which the treatment } \\
\text { takes place. }\end{array}$ \\
\hline Release area & $20 \mathrm{~cm}^{2}$ & $\begin{array}{l}\text { Evaporation takes place from a constant surface area, i.e. } \\
\text { the mixing cup. The surface area is estimated at } 20 \mathrm{~cm}^{2} .\end{array}$ \\
\hline \multicolumn{3}{|c|}{ Dermal, instant application } \\
\hline Surface area & $2 \mathrm{~cm}^{2}$ & Two fingertips \\
\hline Product amount & $50 \mathrm{mg}$ & One drop \\
\hline \multicolumn{3}{|l|}{ Two-component glue } \\
\hline Frequency & 3 year $^{-1}$ & Estimate \\
\hline \multicolumn{3}{|c|}{ Inhalation, evaporation from increasing area } \\
\hline Exposure duration & $240 \mathrm{~min}$ & The subject stays in the room after use \\
\hline Application duration & $30 \mathrm{~min}$ & Estimate \\
\hline Product amount & $20 \mathrm{~g}$ & Product information: gluing a large broken vase \\
\hline Release area & $20 \mathrm{~cm}^{2}$ & $\begin{array}{l}\text { Evaporation takes place from a constant surface area, i.e. } \\
\text { the mixing cup. The surface area is estimated at } 20 \mathrm{~cm}^{2} .\end{array}$ \\
\hline \multicolumn{3}{|c|}{ Dermal, instant application } \\
\hline Surface area & $43 \mathrm{~cm}^{2}$ & $\begin{array}{l}\text { Assuming that approximately } 20 \% \text { of one palm (quarter } \\
\text { of the total surface of the hands }=215 \mathrm{~cm}^{2} \text { ) is exposed, } \\
\text { provides a surface area of } 43 \mathrm{~cm}^{2} .\end{array}$ \\
\hline Product amount & $0.1 \mathrm{~g}$ & $\begin{array}{l}\text { The dermal load is not high, because relatively low } \\
\text { amounts are used. Assuming that two drops are spilled, } \\
\text { a dermal load of } 0.1 \mathrm{~g} \text { can be derived. }\end{array}$ \\
\hline \multicolumn{3}{|c|}{ Two component parquet glue: mixing and loading } \\
\hline Frequency & 3 day $^{-1}$ & 3 day $^{-1}$, once every 8 years \\
\hline \multicolumn{3}{|c|}{ Inhalation, evaporation constant release area } \\
\hline Exposure duration & $10 \mathrm{~min}$ & Estimate \\
\hline Application duration & $10 \mathrm{~min}$ & Estimate \\
\hline Product amount & $7 \mathrm{~kg}$ & $\begin{array}{l}\text { The product amount required for this task is very high ( } 22 \\
\mathrm{~kg} \text {; derived from consumption rate of } 1 \mathrm{~kg} / \mathrm{m}^{2} \text { and surface } \\
\text { area of } 22 \mathrm{~m}^{2} \text { ). The mixing and loading step will be repeated } \\
\text { approximately three times, because the product amount is } \\
\text { simply too much to handle in one step. }\end{array}$ \\
\hline Room volume & $1 \mathrm{~m}^{3}$ & See "Two-component glue: mixing and loading" \\
\hline Release area & $320 \mathrm{~cm}^{2}$ & Surface bucket \\
\hline \multicolumn{3}{|c|}{ Dermal, instant application } \\
\hline Surface area & $215 \mathrm{~cm}^{2}$ & $1 / 4$ of two hands \\
\hline Product amount & $200 \mathrm{mg}$ & Estimate \\
\hline \multicolumn{3}{|c|}{ Parquet glue: gluing on surface } \\
\hline Frequency & $1 / 8$ year $^{-1}$ & Once in eight years \\
\hline \multicolumn{3}{|c|}{ Inhalation, evaporation constant release area } \\
\hline Exposure duration & $480 \mathrm{~min}$ & Estimate, eight hours \\
\hline Application duration & $480 \mathrm{~min}$ & Estimate, eight hours \\
\hline Product amount & $22 \mathrm{~kg}$ & Product information \\
\hline Room volume & $58 \mathrm{~m}^{3}$ & Living room \\
\hline Ventilation rate & $0.5 \mathrm{~h}^{-1}$ & Living room \\
\hline Release area & $1 \mathrm{~m}^{2}$ & $\begin{array}{l}\text { The release area is set equal to the surface area that } \\
\text { can be treated per interval. It is assumed that an } \\
\text { individual treats } 1 \mathrm{~m}^{2} \text { per interval. After that the } \\
\text { surface is covered and the exposure will be negligible } \\
\text { compared with the newly treated surface. These steps } \\
\text { are repeated until the task is completed, resulting in } 22 \\
\text { repetitions. The inhalation exposure is described by } \\
\text { evaporation of the total amount and not the adjusted } \\
\text { amount from a constant surface area, i.e. } 1 \mathrm{~m}^{2} \text {. This } \\
\text { simplification of the model is necessary, because } \\
\text { otherwise depletion of the source can occur. }\end{array}$ \\
\hline
\end{tabular}




\begin{tabular}{|c|c|c|}
\hline & Default Values & Comments \\
\hline \multicolumn{3}{|l|}{ Dermal, constant rate } \\
\hline Surface area & $430 \mathrm{~cm}^{2}$ & $50 \%$ of both hands \\
\hline Contact rate & $30 \mathrm{mg} / \mathrm{min}$ & Default value for downward or to the side painting \\
\hline Release duration & $480 \mathrm{~min}$ & Estimate, eight hours \\
\hline \multicolumn{3}{|c|}{ Parquet glue: floating parquet } \\
\hline Frequency & $1 / 4$ year $^{-1}$ & Once every four years \\
\hline \multicolumn{3}{|c|}{ Inhalation, evaporation constant release area } \\
\hline Exposure duration & $240 \mathrm{~min}$ & Estimate, four hours \\
\hline Application duration & $240 \mathrm{~min}$ & I. e. exposure duration \\
\hline Product amount & $750 \mathrm{~g}$ & $\begin{array}{l}\text { According to the product information } 1 \mathrm{~kg} \text { is sufficient } \\
\text { to cover } 30 \mathrm{~m}^{2} . \text { For an area of } 22 \mathrm{~m}^{2} \text { it is assumed that }\end{array}$ \\
\hline & & 750 grams of glue is used. \\
\hline Room volume & $58 \mathrm{~m}^{3}$ & Living room \\
\hline Ventilation rate & $0.5 \mathrm{~h}^{-1}$ & Living room \\
\hline Release area & $1 \mathrm{~m}^{2}$ & $\begin{array}{l}\text { It is assumed that } 1 \mathrm{~m}^{2} \text { is treated per interval; the } \\
\text { effective release area is then } 1 \mathrm{~m}^{2} . \text { This interval is } \\
\text { repeated } 22 \text { times to complete the task. See "Parquet } \\
\text { glue: gluing on surface" }\end{array}$ \\
\hline \multicolumn{3}{|c|}{ Dermal, instant application } \\
\hline Surface area & $110 \mathrm{~cm}^{2}$ & $50 \%$ of one hand palm \\
\hline Product amount & $0.5 \mathrm{~g}$ & Estimate \\
\hline \multicolumn{3}{|l|}{ Carpet glue } \\
\hline Frequency & $1 / 4$ year $^{-1}$ & Once every four years \\
\hline \multicolumn{3}{|c|}{ Inhalation, evaporation constant release area } \\
\hline Exposure duration & $75 \mathrm{~min}$ & $\begin{array}{l}\text { Assuming that the carpet is already cut to size and is } \\
\text { only moved to fit }\end{array}$ \\
\hline Application duration & $75 \mathrm{~min}$ & I.e. exposure duration \\
\hline Product amount & $9 \mathrm{~kg}$ & $\begin{array}{l}\text { According to product information an average of } 1 \mathrm{~kg} \\
\text { carpet glue per } 2.5 \mathrm{~m}^{2} \text { is used for laying carpets, provid- } \\
\text { ing a total product amount of approximately } 9 \mathrm{~kg} \\
\text { carpet glue }\end{array}$ \\
\hline Room volume & $58 \mathrm{~m}^{3}$ & Living room \\
\hline Ventilation rate & $0.5 \mathrm{~h}^{-1}$ & Living room \\
\hline Release area & $4 \mathrm{~m}^{2}$ & $\begin{array}{l}\text { It is assumed that } 4 \mathrm{~m}^{2} \text { per interval can be treated, } \\
\text { which will take } 15 \text { minutes per effort }\end{array}$ \\
\hline \multicolumn{3}{|l|}{ Dermal, constant rate } \\
\hline Surface area & $110 \mathrm{~cm}^{2}$ & $50 \%$ of one hand palm \\
\hline Contact rate & $30 \mathrm{mg} / \mathrm{min}$ & See "Parquet glue: gluing on surface" \\
\hline Release duration & $75 \mathrm{~min}$ & See "exposure duration" \\
\hline \multicolumn{3}{|c|}{ Tile glue: mixing and loading } \\
\hline Frequency & 5 day $^{-1}$ & Once every 2 years \\
\hline \multicolumn{3}{|l|}{ Dermal, constant rate } \\
\hline Surface area & $430 \mathrm{~cm}^{2}$ & $50 \%$ of both hands \\
\hline Contact rate & $0.33 \mathrm{mg} / \mathrm{min}$ & Default value for large tasks \\
\hline Release duration & $1.33 \mathrm{~min}$ & Estimate \\
\hline \multicolumn{3}{|l|}{ Tile glue } \\
\hline Frequency & 0.5 year $^{-1}$ & Estimate \\
\hline \multicolumn{3}{|c|}{ Inhalation, evaporation constant release area } \\
\hline Exposure duration & $360 \mathrm{~min}$ & Estimate, six hours \\
\hline Application time & $360 \mathrm{~min}$ & Estimate, six hours \\
\hline Product amount & $15 \mathrm{~kg}$ & $\begin{array}{l}\text { Product information. The use of glue is on average } 1-2 \\
\mathrm{~kg} / \mathrm{m}^{2} \text {. Assuming an average use of } 1.5 \mathrm{~kg} / \mathrm{m}^{2} \text {, this yields a } \\
\text { total amount of } 15 \mathrm{~kg} \text { tile glue to be used on } 10 \mathrm{~m}^{2} \text {. }\end{array}$ \\
\hline Room volume & $10 \mathrm{~m}^{3}$ & Bathroom \\
\hline Ventilation rate & $2 \mathrm{~h}^{-1}$ & Bathroom \\
\hline Release area & $1 \mathrm{~m}^{2}$ & $\begin{array}{l}\text { This is the surface a subject is assumed to cover per } \\
\text { interval. }\end{array}$ \\
\hline \multicolumn{3}{|l|}{ Dermal, constant rate } \\
\hline Surface area & $430 \mathrm{~cm}^{2}$ & Two hand palms; $50 \%$ of both hands \\
\hline Contact rate & $30 \mathrm{mg} / \mathrm{min}$ & See "Parquet glue: gluing on surface" \\
\hline Release duration & $360 \mathrm{~min}$ & Estimate \\
\hline \multicolumn{3}{|c|}{ Wall paper glue: mixing and loading } \\
\hline Frequency & 0.5 year $^{-1}$ & Estimate; once every two years \\
\hline \multicolumn{3}{|l|}{ Dermal, constant rate } \\
\hline Surface area & $430 \mathrm{~cm}^{2}$ & $50 \%$ of both hands \\
\hline Contact rate & $0.033 \mathrm{mg} / \mathrm{min}$ & Default value for small tasks \\
\hline Release duration & $1.33 \mathrm{~min}$ & Estimate \\
\hline
\end{tabular}




\begin{tabular}{|c|c|c|}
\hline & Default Values & Comments \\
\hline \multicolumn{3}{|l|}{ Wall paper glue } \\
\hline Frequency & 0.5 year $^{-1}$ & Estimate; once every two years \\
\hline \multicolumn{3}{|l|}{ Dermal, constant rate } \\
\hline \multirow[t]{2}{*}{ Surface area } & $860 \mathrm{~cm}^{2}$ & $\begin{array}{l}\text { Both hands. The spreading of the glue, folding and } \\
\text { hanging of wall paper will cause spills on both hands. }\end{array}$ \\
\hline & & $\begin{array}{l}\text { The dermal load can be significant, depending on how } \\
\text { the subject handles the wall paper. Since wall paper } \\
\text { glue is not considered harmful and easy to remove } \\
\text { afterwards, subjects tend to be more careless. }\end{array}$ \\
\hline Contact rate & $30 \mathrm{mg} / \mathrm{min}$ & See "Parquet glue: gluing on surface" \\
\hline Release duration & $240 \min$ & Estimate, six hours \\
\hline \multicolumn{3}{|l|}{ Hot melt adhesive } \\
\hline Frequency & 1 month $^{-1}$ & $\begin{array}{l}\text { Estimate; once per month. Gluing the soles of the shoes } \\
\text { with a hot melt adhesive }\end{array}$ \\
\hline \multicolumn{3}{|l|}{ Inhalation, instant release } \\
\hline Exposure duration & $25 \mathrm{~min}$ & $\begin{array}{l}\text { In total, an application duration of } 25 \text { minutes will be } \\
\text { taken into account which is spread over a longer period } \\
\text { of time (gluing occurs with intervals). }\end{array}$ \\
\hline Product amount & $65 \mathrm{~g}$ & One glue bar \\
\hline \multicolumn{3}{|l|}{ Dermal, instant application } \\
\hline Surface area & $43 \mathrm{~cm}^{2}$ & $20 \%$ of a hand palm \\
\hline Product amount & $100 \mathrm{mg}$ & $\begin{array}{l}\text { Estimate. The dermal load will be low, because the glue } \\
\text { is still solid at room temperature. The glue is more or } \\
\text { less rubbed off the glue bar. }\end{array}$ \\
\hline \multicolumn{3}{|l|}{ Glue spray } \\
\hline Frequency & 1 month $^{-1}$ & Estimate; gluing a poster to a wall or door, or put in a frame \\
\hline \multicolumn{3}{|l|}{ Inhalation, spray model } \\
\hline Spray duration & $170 \mathrm{sec}$ & $\begin{array}{l}\text { The surface of the poster is assumed to be } 1.5 \mathrm{~m}^{2} \text {. } \\
\text { According to product information } 300 \mathrm{ml}(255 \mathrm{~g} \text {; product } \\
\left.\text { density is } 0.85 \mathrm{~g} / \mathrm{cm}^{3}\right) \text { is required to glue the poster. Using } \\
\text { the mass generation rate from Table } 11.2 .2 .1 . \text { ( } 1.5 \mathrm{~g} / \mathrm{sec}) \\
\text { provides a spraying duration of } 170 \text { seconds }\end{array}$ \\
\hline Exposure duration & $240 \min$ & $\begin{array}{l}\text { The exposure duration is the time that a person stays in } \\
\text { the room duringand after the task }\end{array}$ \\
\hline Mass generation rate & $1.5 \mathrm{~g} / \mathrm{sec}$ & See section 11.2.2. "Cleaning Products" \\
\hline Airborne fraction & $1 \mathrm{~g} / \mathrm{g}$ & See section 11.2.2. "Cleaning Products" \\
\hline Weight fraction non-volatile & 0.16 & Product information \\
\hline Density non-volatile & $1.3 \mathrm{~g} / \mathrm{cm}^{3}$ & See section 11.2.2. "Cleaning Products" \\
\hline $\begin{array}{l}\text { Initial droplet distribution - } \\
\text { median (CV) }\end{array}$ & $40 \mu \mathrm{m}(0.4)$ & See section 11.2.2. "Cleaning Products" \\
\hline \multicolumn{3}{|l|}{ Dermal, constant rate } \\
\hline Surface area & $430 \mathrm{~cm}^{2}$ & $50 \%$ of both hands \\
\hline Contact rate & $100 \mathrm{mg} / \mathrm{min}$ & $\begin{array}{l}\text { Default value for overhead painting with low viscosity } \\
\text { products }\end{array}$ \\
\hline Release duration & $170 \mathrm{sec}$ & See "spray duration" \\
\hline \multicolumn{3}{|l|}{ Joint sealant } \\
\hline Frequency & 3 year $^{-1}$ & Estimate; based on one study \\
\hline \multicolumn{3}{|c|}{ Inhalation, evaporation from increasing area } \\
\hline Exposure duration & $45 \mathrm{~min}$ & Estimate; includes time of finishing up after use \\
\hline Application duration & $30 \mathrm{~min}$ & Estimate \\
\hline Product amount & $75 \mathrm{~g}$ & Estimate; silicon based sealant \\
\hline Room volume & $10 \mathrm{~m}^{3}$ & Bathroom \\
\hline Ventilation rate & $2 h^{-1}$ & Bathroom \\
\hline Release area & $250 \mathrm{~cm}^{2}$ & $\begin{array}{l}5 \mathrm{~m} * 5 \mathrm{~mm} \text { (joints between bathtub, shower cabinet, } \\
\text { or washstands and wall) }\end{array}$ \\
\hline \multicolumn{3}{|l|}{ Dermal, constant rate } \\
\hline Surface area & $2 \mathrm{~cm}^{2}$ & $\begin{array}{l}\text { The dermal exposure area is the tip of one finger ( } 1 \\
\mathrm{~cm}^{2} \text { ). Due to the amount of surplus sealant the expo- } \\
\text { sure area is set at } 2 \mathrm{~cm}^{2} \text { instead of just the fingertip. }\end{array}$ \\
\hline Contact rate & $50 \mathrm{mg} / \mathrm{min}$ & $\begin{array}{l}\text { Using the constant rate model the constant rate is } \\
\text { calculated to be } 50 \mathrm{mg} / \mathrm{min} \text { ( } 1.5 \mathrm{~g} \text { divided by release } \\
\text { duration of } 30 \mathrm{~min})\end{array}$ \\
\hline Release duration & $30 \mathrm{~min}$ & See "application duration" \\
\hline
\end{tabular}




\begin{tabular}{|c|c|c|}
\hline & Default Values & Comments \\
\hline \multicolumn{3}{|l|}{ Assembly sealant } \\
\hline Frequency & 1 year $^{-1}$ & Estimate \\
\hline \multicolumn{3}{|c|}{ Inhalation, evaporation from increasing area } \\
\hline Exposure duration & $240 \mathrm{~min}$ & $\begin{array}{l}\text { An application duration of } 30 \text { minutes is chosen here. } \\
\text { The time a subject stays in the room after the DIY task } \\
\text { is } 4 \text { hours which will be used in the default scenario. }\end{array}$ \\
\hline Application duration & $30 \mathrm{~min}$ & Estimate \\
\hline Product amount & $390 \mathrm{~g}$ & Product information \\
\hline Release area & $1.5 \mathrm{~m}^{2}$ & Estimate, sealing a wooden frame \\
\hline \multicolumn{3}{|c|}{ Dermal, instant application } \\
\hline Surface area & $43 \mathrm{~cm}^{2}$ & $20 \%$ of one hand palm \\
\hline Product amount & $0.5 \mathrm{~g}$ & Estimate \\
\hline \multicolumn{3}{|c|}{ General filler from powder: mixing and loading } \\
\hline Frequency & 2 year $^{-1}$ & Estimate \\
\hline \multicolumn{3}{|l|}{ Inhalation } \\
\hline Inhalation of dust & $0.3 \mu \mathrm{g}$ & $\begin{array}{l}\text { Calculated by the contact rate of } 0.25 \mu \mathrm{g} / \mathrm{min} \text { and } \\
\text { exposure duration of } 1.33 \mathrm{~min}\end{array}$ \\
\hline \multicolumn{3}{|l|}{ Dermal, constant rate } \\
\hline Surface area & $430 \mathrm{~cm}^{2}$ & $50 \%$ of both hands \\
\hline Contact rate & $0.033 \mathrm{mg} / \mathrm{min}$ & Default value for small tasks \\
\hline Release duration & $1.33 \mathrm{~min}$ & Estimate \\
\hline \multicolumn{3}{|c|}{ General filler from powder ${ }^{a}$} \\
\hline Frequency & 2 year $^{-1}$ & Estimate \\
\hline \multicolumn{3}{|c|}{ Dermal, instant application } \\
\hline Surface area & $430 \mathrm{~cm}^{2}$ & $50 \%$ of both hands \\
\hline Product amount & $0.25 \mathrm{~g}$ & $\begin{array}{l}\text { Filler is spilled on hands, while putting the filler in the } \\
\text { hole. The spilled amount will be low, because working } \\
\text { with fillers is relatively easy. The dermal load is esti- } \\
\text { mated at } 0.25 \mathrm{~g} \text {, which is } 0.1 \% \text { (estimation) of the total } \\
\text { product amount. }\end{array}$ \\
\hline Exposure duration & $15 \mathrm{~min}$ & Estimate \\
\hline \multicolumn{3}{|c|}{ Large hole filler from powder: mixing and loading } \\
\hline Frequency & 1 year $^{-1}$ & Estimate \\
\hline \multicolumn{3}{|l|}{ Inhalation } \\
\hline Inhalation of dust & $0.3 \mu \mathrm{g}$ & $\begin{array}{l}\text { Calculated by the contact rate of } 0.25 \mu \mathrm{g} / \mathrm{min} \text { and } \\
\text { exposure duration of } 1.33 \mathrm{~min}\end{array}$ \\
\hline \multicolumn{3}{|l|}{ Dermal, constant rate } \\
\hline Surface area & $430 \mathrm{~cm}^{2}$ & $50 \%$ of both hands \\
\hline Contact rate & $0.033 \mathrm{mg} / \mathrm{min}$ & Default value for small tasks \\
\hline Release duration & $1.33 \mathrm{~min}$ & Estimate \\
\hline \multicolumn{3}{|l|}{ Large hole fillers ${ }^{a}$} \\
\hline Frequency & 1 year $^{-1}$ & Estimate \\
\hline \multicolumn{3}{|c|}{ Dermal, instant application } \\
\hline Surface area & $430 \mathrm{~cm}^{2}$ & One finger \\
\hline Product amount & $0.5 \mathrm{~g}$ & Estimate \\
\hline Exposure duration & $30 \mathrm{~min}$ & Estimate; application duration \\
\hline \multicolumn{3}{|c|}{ Fillers/putty from tube } \\
\hline Frequency & 3 year $^{-1}$ & Estimate \\
\hline \multicolumn{3}{|c|}{ Inhalation, evaporation from increasing area } \\
\hline Exposure duration & $240 \mathrm{~min}$ & $\begin{array}{l}\text { Estimate, assuming that the subject stays in the room for } 4 \\
\text { hours in total, the exposure duration will be } 4 \text { hours. }\end{array}$ \\
\hline Application duration & $20 \mathrm{~min}$ & Estimate \\
\hline Product amount & $40 \mathrm{~g}$ & $\begin{array}{l}\text { It is estimated that this DIY task will require } 40 \text { grams to } \\
\text { fill the holes. }\end{array}$ \\
\hline Release area & $200 \mathrm{~cm}^{2}$ & $\begin{array}{l}\text { The surface area is determined by summing each cover } \\
\text { of a screw hole (of an entire bookcase). This is estimat- } \\
\text { ed at } 5 \mathrm{~cm}^{2} \text { per screw hole, assuming } 40 \text { screw holes; a } \\
\text { total surface area of } 200 \mathrm{~cm}^{2} \text { is provided. }\end{array}$ \\
\hline \multicolumn{3}{|c|}{ Dermal, instant application } \\
\hline Surface area & $22 \mathrm{~cm}^{2}$ & $5 \%$ of one hand \\
\hline Product amount & $0.05 \mathrm{~g}$ & Estimate \\
\hline
\end{tabular}




\begin{tabular}{|c|c|c|}
\hline & Default Values & Comments \\
\hline \multicolumn{3}{|c|}{ Two-component filler: mixing and loading } \\
\hline Frequency & 2 year $^{-1}$ & Estimate \\
\hline \multicolumn{3}{|c|}{ Inhalation, evaporation constant release area } \\
\hline Exposure duration & $5 \mathrm{~min}$ & Estimate \\
\hline Application duration & $5 \mathrm{~min}$ & Estimate \\
\hline Product amount & $200 \mathrm{~g}$ & Estimate \\
\hline Room volume & $1 \mathrm{~m}^{3}$ & $\begin{array}{l}\text { "Room volume" is interpreted here as personal space: a } \\
\text { small area of } 1 \mathrm{~m}^{3} \text { around the user. A small area around } \\
\text { the user is relevant for the inhalation exposure of the } \\
\text { user for the short use duration in which the mixing } \\
\text { takes place. }\end{array}$ \\
\hline Release area & $100 \mathrm{~cm}^{2}$ & Estimate \\
\hline \multicolumn{3}{|l|}{ Dermal, instant application } \\
\hline Surface area & $2 \mathrm{~cm}^{2}$ & Two fingertips \\
\hline Product amount & $20 \mathrm{mg}$ & Estimate \\
\hline \multicolumn{3}{|l|}{ Two-component filler } \\
\hline Frequency & 2 year $^{-1}$ & Estimate \\
\hline \multicolumn{3}{|c|}{ Inhalation, evaporation from increasing area } \\
\hline Exposure duration & $240 \mathrm{~min}$ & Estimate \\
\hline Application duration & $30 \mathrm{~min}$ & Estimate \\
\hline Product amount & $200 \mathrm{~g}$ & Estimate \\
\hline Release area & $50 \mathrm{~cm}^{2}$ & $\begin{array}{l}\text { The surface from which the chemicals can evaporate is } \\
\text { estimated to be } 50 \mathrm{~cm}^{2}\left(5 \times 10 \mathrm{~cm}^{2}\right) \text { (Repairing all spots } \\
\text { in the decayed wood of a window frame) }\end{array}$ \\
\hline \multicolumn{3}{|l|}{ Dermal, instant application } \\
\hline Surface area & $22 \mathrm{~cm}^{2}$ & $5 \%$ of one hand due to spills \\
\hline Product amount & $0.2 \mathrm{~g}$ & $\begin{array}{l}\text { The dermal load is estimated at } 0.2 \mathrm{~g} \text { which is } 0.1 \% \\
\text { (assumption) of the total product amount }\end{array}$ \\
\hline \multicolumn{3}{|l|}{ Putty spray } \\
\hline Frequency & 1 year $^{-1}$ & Estimate \\
\hline \multicolumn{3}{|l|}{ Inhalation, spray model } \\
\hline Spray duration & $135 \mathrm{sec}$ & $\begin{array}{l}\text { For this task it is assumed that } 200 \mathrm{~g} \text { is required to } \\
\text { cover a surface area of } 500 \mathrm{~cm}^{2} . \text { A mass generation rate } \\
\text { of } 1.5 \mathrm{~g} / \mathrm{sec} \text { provides spray duration of } 135 \text { seconds } \\
\text { (rounded off number; } 3 \times \text { spraying to cover the scratch- } \\
\text { es provides a spraying duration of } 45 \text { seconds). }\end{array}$ \\
\hline Exposure duration & $30 \mathrm{~min}$ & $\begin{array}{l}\text { After completing the task the subject will leave the garage, } \\
\text { making the total exposure duration half an hour. }\end{array}$ \\
\hline Room volume & $34 \mathrm{~m}^{3}$ & Garage \\
\hline Ventilation rate & $1.5 \mathrm{~h}^{-1}$ & Garage \\
\hline Mass generation rate & $1.5 \mathrm{~g} / \mathrm{sec}$ & See section 11.2.2. "Cleaning Products" \\
\hline Airborne fraction & $1 \mathrm{~g} / \mathrm{g}$ & See section 11.2.2. "Cleaning Products" \\
\hline Weight fraction non-volatile & 0.3 & $\begin{array}{l}\text { The weight fraction of nonvolatiles is estimated to be } \\
30 \% \text {, twice as high as for glue spray, because it is as- } \\
\text { sumed that putty requires a higher percentage of solids }\end{array}$ \\
\hline Density non-volatile & $1.3 \mathrm{~g} / \mathrm{cm}^{3}$ & See section 11.2.2. "Cleaning Products" \\
\hline $\begin{array}{l}\text { Initial droplet distribution - } \\
\text { median (CV) }\end{array}$ & $40 \mu \mathrm{m}(0.4)$ & See section 11.2.2. "Cleaning Products" \\
\hline \multicolumn{3}{|l|}{ Dermal, constant rate } \\
\hline Surface area & $860 \mathrm{~cm}^{2}$ & Both hands \\
\hline Contact rate & $100 \mathrm{mg} / \mathrm{min}$ & $\begin{array}{l}\text { Default value for overhead painting with low viscosity } \\
\text { products }\end{array}$ \\
\hline Release duration & $\begin{array}{l}135 \sec (45 \\
\left.s^{*} 3\right)\end{array}$ & See "spray duration" \\
\hline \multicolumn{3}{|c|}{ Floor equalizer: mixing and loading } \\
\hline Frequency & 3 day $^{-1}$ & $\begin{array}{l}3 \text { day }^{-1} \text { once every } 2 \text { years. The product amount }(25 \mathrm{~kg}) \\
\text { is not mixed all at once. Therefore the frequency of the } \\
\text { mixing and loading process is three per task. }\end{array}$ \\
\hline \multicolumn{3}{|l|}{ Inhalation } \\
\hline Inhalation of dust & $3.0 \mu \mathrm{g}$ & $\begin{array}{l}\text { Inhalation exposure to such large amounts was calcu- } \\
\text { lated by the rate of contact: } 2.5 \mu \mathrm{g} / \mathrm{min} \text { x exposure } \\
\text { duration of } 1.33 \text { minutes yields an inhalation exposure } \\
\text { of } 3.0 \mu \mathrm{g} \text {. }\end{array}$ \\
\hline \multicolumn{3}{|l|}{ Dermal, constant rate } \\
\hline Surface area & $430 \mathrm{~cm}^{2}$ & $50 \%$ of both hands \\
\hline Contact rate & $0.33 \mathrm{mg} / \mathrm{min}$ & Default value for large tasks \\
\hline Release duration & $1.33 \mathrm{~min}$ & Estimate \\
\hline
\end{tabular}




\begin{tabular}{|c|c|c|}
\hline & Default Values & Comments \\
\hline \multicolumn{3}{|l|}{ Floor equalizer } \\
\hline Frequency & 0.5 year $^{-1}$ & Estimate \\
\hline \multicolumn{3}{|c|}{ Dermal, instant application } \\
\hline Surface area & $860 \mathrm{~cm}^{2}$ & Both hands \\
\hline Product amount & $2 \mathrm{~g}$ & Estimate \\
\hline Exposure duration & $30 \mathrm{~min}$ & Estimate \\
\hline \multicolumn{3}{|l|}{ Wall plaster } \\
\hline Frequency & 0.2 year $^{-1}$ & Estimate; once every five years \\
\hline \multicolumn{3}{|l|}{ Dermal, constant rate } \\
\hline Surface area & $1900 \mathrm{~cm}^{2}$ & Hands and forearms \\
\hline Contact rate & $50 \mathrm{mg} / \mathrm{min}$ & Estimate \\
\hline Release duration & $120 \mathrm{~min}$ & Estimate \\
\hline \multicolumn{3}{|c|}{ General coating on a floor } \\
\hline Frequency & 0.33 year $^{-1}$ & Estimate; once per three years \\
\hline \multicolumn{3}{|c|}{ Inhalation, evaporation from increasing area } \\
\hline Exposure duration & $60 \mathrm{~min}$ & $\begin{array}{l}\text { Estimate; the person will not stay in the garage after } \\
\text { application }\end{array}$ \\
\hline Application duration & $60 \mathrm{~min}$ & Estimate \\
\hline Product amount & $3 \mathrm{~kg}$ & $\begin{array}{l}\text { The scenario is a garage with a surface area of } 15 \mathrm{~m}^{2} \text {. } \\
\text { The consumption rate of the coating is according to } \\
\text { product information } 0.15 \text { litre per square metre. In } \\
\text { total } 2.5 \text { litres ( } 3 \mathrm{~kg} \text { using } 1.2 \mathrm{~g} / \mathrm{cm}^{3} \text { ) of coating is } \\
\text { required for one layer }\end{array}$ \\
\hline Room volume & $34 \mathrm{~m}^{3}$ & Garage \\
\hline Ventilation rate & $1.5 \mathrm{~h}^{-1}$ & Garage \\
\hline Release area & $15 \mathrm{~m}^{2}$ & Garage \\
\hline Temperature & $15^{\circ} \mathrm{C}$ & Estimate \\
\hline \multicolumn{3}{|c|}{ Dermal, instant application } \\
\hline Surface area & $108 \mathrm{~cm}^{2}$ & $50 \%$ of one hand palm \\
\hline Product amount & $0.25 \mathrm{~g}$ & Estimate; only due to spills \\
\hline \multicolumn{3}{|l|}{ Gutter coating } \\
\hline Frequency & 0.1 year $^{-1}$ & Estimate; once per 10 years \\
\hline \multicolumn{3}{|c|}{ Dermal, instant application } \\
\hline Surface area & $430 \mathrm{~cm}^{2}$ & $\begin{array}{l}\text { It is assumed that fingers on both hands will be exposed } \\
\text { when the glass cloth is put in place. The surface area is } \\
\text { set at half the area covered by the two hands }\left(430 \mathrm{~cm}^{2}\right)\end{array}$ \\
\hline Product amount & $0.25 \mathrm{~g}$ & $\begin{array}{l}\text { The dermal load is estimated to be } 250 \mathrm{mg} \text { due to the } \\
\text { fact that the product will stick to one's hand. The } \\
\text { dermal load is not related to the amount product used } \\
\text { (=approx. } 1 \mathrm{~kg} \text { ). }\end{array}$ \\
\hline Exposure duration & $240 \mathrm{~min}$ & I.e. application duration; half a working day \\
\hline \multicolumn{3}{|l|}{ Paint remover } \\
\hline Frequency & 1 year $^{-1}$ & Estimate; once a year \\
\hline \multicolumn{3}{|c|}{ Inhalation, evaporation from increasing area } \\
\hline Exposure duration & $60 \mathrm{~min}$ & Estimate; i.e. application duration \\
\hline Application duration & $60 \mathrm{~min}$ & Estimate \\
\hline Product amount & $1 \mathrm{~kg}$ & $\begin{array}{l}\text { The surface area is approximately } 2 \mathrm{~m}^{2} \text { (one side of the } \\
\text { door) for which } 1 \mathrm{I} \text { is required (= } 1 \mathrm{~kg} \text { ). }\end{array}$ \\
\hline Release area & $2 \mathrm{~m}^{2}$ & See "product amount" \\
\hline \multicolumn{3}{|c|}{ Dermal, instant application } \\
\hline Surface area & $430 \mathrm{~cm}^{2}$ & Both hand palms \\
\hline Product amount & $0.5 \mathrm{~g}$ & Estimate; from pulps and remnants \\
\hline \multicolumn{3}{|l|}{ Glue remover } \\
\hline Frequency & $1 / 4$ year $^{-1}$ & Estimate \\
\hline \multicolumn{3}{|c|}{ Inhalation, evaporation from increasing area } \\
\hline Exposure duration & $240 \mathrm{~min}$ & $\begin{array}{l}\text { The total exposure duration is set at four hours, because } \\
\text { after the task the subject will not stay in the same "room". }\end{array}$ \\
\hline Application duration & $240 \mathrm{~min}$ & $\begin{array}{l}\text { The application duration is based on the pouring and } \\
\text { spreading of the remover, the activation time, and the } \\
\text { time needed to scratch off the old glue. The latter will } \\
\text { consume a lot of time. }\end{array}$ \\
\hline Product amount & $2 \mathrm{~kg}$ & $\begin{array}{l}\text { Product information; it is assumed that the stairs are } \\
\text { stripped of their carpet and carpet glue. The surface of the } \\
\text { stairs is approximately } 5 \mathrm{~m}^{2} \text {. Using the product information } \\
\left(0.35 \mathrm{l} / \mathrm{m}^{2}\right) \text { provides a product amount of about } 2 \mathrm{~kg} \text {. }\end{array}$ \\
\hline Room volume & $30 \mathrm{~m}^{3}$ & Estimate; stairs (large volume) \\
\hline Ventilation rate & $1.5 \mathrm{~h}^{-1}$ & Estimate \\
\hline Release area & $5 \mathrm{~m}^{2}$ & Estimate; surface of the stairs \\
\hline
\end{tabular}




\begin{tabular}{|c|c|c|}
\hline & Default Values & Comments \\
\hline \multicolumn{3}{|l|}{ Dermal, constant rate } \\
\hline Surface area & $230 \mathrm{~cm}^{2}$ & The fingers of both hands \\
\hline Contact rate & $30 \mathrm{mg} / \mathrm{min}$ & Default value for downward or to the side painting \\
\hline Release duration & $240 \mathrm{~min}$ & I.e. application duration \\
\hline \multicolumn{3}{|c|}{ Wall paper remover: mixing and loading } \\
\hline Frequency & 0.5 year $^{-1}$ & Estimate \\
\hline \multicolumn{3}{|l|}{ Dermal, instant application } \\
\hline Product amount on skin & $\begin{array}{l}10 \\
\text { mg/operation }\end{array}$ & $\begin{array}{l}\text { As a default for } 40 \mathrm{~m}^{2} \text { the total product amount is set at } \\
3 \text { litres of suspension ( } 150 \mathrm{ml} \text { wall paper remover in } 3 \mathrm{I} \\
\text { lukewarm water, equal to approximately } 3 \mathrm{~kg} \text {; dilution } \\
\text { factor }=20 \text { ). The time needed to make the suspension } \\
\text { is approximately five minutes. The } 75^{\text {th }} \text { percentiles for } \\
\text { dermal exposure during mixing and loading are given } \\
\text { for } 1 \text { litre and } 2 \text { litre containers i.e. } 0.01 \mathrm{ml} \text { (undiluted } \\
\text { product) per operation. A default for mixing and } \\
\text { loading of wall paper remover of } 0.01 \mathrm{ml} \text { per operation } \\
\text { (=10 mg/operation) will be used. }\end{array}$ \\
\hline Surface area & $2 \mathrm{~cm}^{2}$ & $\begin{array}{l}\text { Two fingertips. It is emphasized here that the dermal } \\
\text { exposure results from liquid spills around the opening } \\
\text { of the bottle and spatters of the product i.e. small } \\
\text { product amount. }\end{array}$ \\
\hline \multicolumn{3}{|l|}{ Wall paper remover } \\
\hline Frequency & 0.5 year $^{-1}$ & Estimate \\
\hline \multicolumn{3}{|l|}{ Dermal, instant application } \\
\hline Surface area & $1900 \mathrm{~cm}^{2}$ & $\begin{array}{l}\text { The aqueous liquid easily runs down the hands and } \\
\text { forearms. The dermal exposure area is therefore set at } \\
1900 \mathrm{~cm}^{2} \text {, which equals the surface area of both hands } \\
\text { and forearms }\end{array}$ \\
\hline Weight fraction dilution & $0.2 * W_{f}$ & Dilution factor $=20$ \\
\hline Product amount dilution & $19 \mathrm{~g}$ & $\begin{array}{l}\text { It is assumed that suspension spilled or run off from the } \\
\text { sponge during use forms a layer on the skin of } 1 \mathrm{~mm} \text {. } \\
\text { The volume on the skin is then } 19 \mathrm{~cm}^{3} \text {, which equals a } \\
\text { product amount of } 19 \mathrm{~g} \text { (product density is assumed to } \\
\text { be } 1.0 \mathrm{~g} / \mathrm{cm}^{3} \text { ) }\end{array}$ \\
\hline \multicolumn{3}{|l|}{ Sealant/foam remover } \\
\hline Frequency & 5 year $^{-1}$ & Estimate \\
\hline \multicolumn{3}{|c|}{ Inhalation, evaporation from increasing area } \\
\hline Exposure duration & $180 \mathrm{~min}$ & Estimate; includes the cleaning up after application \\
\hline Application duration & $120 \mathrm{~min}$ & Estimate \\
\hline Product amount & $100 \mathrm{~g}$ & $\begin{array}{l}\text { No information is available on the rate of use of the } \\
\text { product; therefore an estimated use of } 100 \mathrm{ml} \text { (assum- } \\
\text { ing a product density of } 1.0 \mathrm{~g} / \mathrm{cm}^{3} \text { provides } 100 \mathrm{~g} \text { ) }\end{array}$ \\
\hline Room volume & $10 \mathrm{~m}^{3}$ & Bathroom \\
\hline Ventilation rate & $2 \mathrm{~h}^{-1}$ & Bathroom \\
\hline Release area & $250 \mathrm{~cm}^{2}$ & From $5 \mathrm{~m} \times 5 \mathrm{~mm}$ joint \\
\hline \multicolumn{3}{|l|}{ Dermal, instant application } \\
\hline Surface area & $5 \mathrm{~cm}^{2}$ & The fingertips of one hand \\
\hline Product amount & $0.1 \mathrm{~g}$ & $0.1 \%$ of the total amount used \\
\hline \multicolumn{3}{|c|}{ Insulation foam - active agents (PU/MDI) and solvents } \\
\hline Frequency & 0.2 year $^{-1}$ & $\begin{array}{l}\text { Estimate; once per five years. A standard DIY task } \\
\text { concerning insulation foam is the insulation of the attic } \\
\text { to avoid heat loss and prevent draught }\end{array}$ \\
\hline \multicolumn{3}{|l|}{ Inhalation, instant release } \\
\hline Exposure duration & $30 \mathrm{~min}$ & I.e. application duration \\
\hline Application duration & $30 \mathrm{~min}$ & Estimate \\
\hline Total product amount & $825 \mathrm{~g}$ & $\begin{array}{l}\text { The product amount is } 750 \mathrm{ml} \text { which equals to } 825 \mathrm{~g} \\
\text { when } 1.10 \mathrm{~g} / \mathrm{cm}^{3} \text { is considered as product density (prod- } \\
\text { uct information). One spray can provide up to } 40 \text { I of } \\
\text { foam, which is considered sufficient to insulate the attic. }\end{array}$ \\
\hline Room volume & $57.5 \mathrm{~m}^{3}$ & $\begin{array}{l}\text { In the General Fact Sheet (RIVM, 2006a) a surface area } \\
\text { for the attic is provided: } 23 \mathrm{~m}^{2} . \text { Taking } 2.5 \mathrm{~m} \text { for the } \\
\text { height of the attic provides } 57.5 \mathrm{~m}^{3}\end{array}$ \\
\hline Ventilation rate & $1.5 \mathrm{~h}^{-1}$ & Estimate \\
\hline \multicolumn{3}{|l|}{ Dermal, instant application } \\
\hline Surface area & $1900 \mathrm{~cm}^{2}$ & Both hands and forearms \\
\hline Product amount & $0.25 \mathrm{~g}$ & Estimate; minimal amount \\
\hline
\end{tabular}




\begin{tabular}{|c|c|c|}
\hline & Default Values & Comments \\
\hline \multicolumn{3}{|l|}{ Joint colour } \\
\hline Frequency & 1 year $^{-1}$ & Estimate \\
\hline \multicolumn{3}{|c|}{ Inhalation, evaporation from increasing area } \\
\hline Exposure duration & $120 \mathrm{~min}$ & I.e. application duration \\
\hline Application duration & $120 \mathrm{~min}$ & $\begin{array}{l}\text { Application and cleaning (i.e. removing surplus joint } \\
\text { colour) }\end{array}$ \\
\hline Product amount & $100 \mathrm{~g}$ & $\begin{array}{l}\text { Per litre product, } 40-60 \mathrm{~m}^{2} \text { can be treated. A reasona- } \\
\text { ble worst case considered for this product amount is } \\
100 \mathrm{ml}(=100 \mathrm{~g}) \text { for } 4 \mathrm{~m}^{2}\end{array}$ \\
\hline Room volume & $10 \mathrm{~m}^{3}$ & Bathroom \\
\hline Ventilation rate & $2 h^{-1}$ & Bathroom \\
\hline Release area & $4 \mathrm{~m}^{2}$ & Shower cabinet and walls \\
\hline \multicolumn{3}{|c|}{ Dermal, instant application } \\
\hline Surface area & $1900 \mathrm{~cm}^{2}$ & $\begin{array}{l}\text { Both forearms and hands (because water can run down } \\
\text { one's arm) }\end{array}$ \\
\hline Product amount & $0.5 \mathrm{~g}$ & $0.5 \%$ of the product \\
\hline
\end{tabular}

a) Inhalatory exposure is expected to be negligible, because the product does not contain constituents which are likely to evaporate except water.

Source: Modified from the Do-it-yourself Products Fact Sheet, RIVM (2007a).

\subsubsection{Paint products}

In the Paint Products Fact Sheet, RIVM (2007b), paint products are classified into product categories, which are drawn up according to the type product and the application method, that is, "Brush and roller painting" and "Spray painting". According to the Paint Products Fact Sheet, RIVM (2007b), there is a relationship between the type of paint, use duration and product amount. Spray cans with paint are usually applied during a short time. Latex wall paint is usually applied for large surfaces in larger quantities and the application duration will be relatively long. The quantity of paint used per unit of time when painting a chair or a window frame will be much smaller than when painting a lathed wall or varnish a floor. If paint is sprayed, aerosol particles are formed which can be inhaled, which may lead to inhalation exposure to non-volatile compounds present in the paint (RIVM, 2007b).

Several models and parameters have already been discussed in the previous sections. It is assumed that the body weight is $65 \mathrm{~kg}$ (default values for adults (RIVM, 2006a)) and the room temperature is $20^{\circ} \mathrm{C}$. Table 11.8 presents default values for brush and roller painting and Table 11.9 presents default values for spray painting. For description of the parameters included in the spray model, see "cosmetics" and "cleaning products". 
Table 11.8: Default Values for Brush and Roller Painting

\begin{tabular}{|c|c|c|}
\hline & Default value & Comment \\
\hline \multicolumn{3}{|c|}{ Brush / roller painting, solvent rich paint ${ }^{a)}$} \\
\hline Frequency & 1 year $^{-1}$ & $\begin{array}{l}\text { Usage } 2 \text { times a year one or a few days after each other; } \\
\text { once per } 2 \text { years }\end{array}$ \\
\hline \multicolumn{3}{|c|}{ Inhalation, evaporation from increasing area } \\
\hline Exposure duration & $132 \mathrm{~min}$ & $\begin{array}{l}\text { The exposure duration is set at } 1.1 \text { times the application } \\
\text { duration, to account for the clean-up time; it is assumed } \\
\text { that the person leaves the room after application and } \\
\text { cleaning up }\end{array}$ \\
\hline Application duration & $120 \mathrm{~min}$ & Estimate \\
\hline Product amount & $1000 \mathrm{~g}$ & $\begin{array}{l}\text { For painting a wooden wall of } 10 \mathrm{~m}^{2}, 0,833 \mathrm{I} \text { or } 1000 \mathrm{~g} \\
\text { paint is necessary (paint coverage } 12 \mathrm{~m}^{2} / \mathrm{l} \text {, density } \\
1.2 \mathrm{~g} / \mathrm{cm}^{3} \text { ). }\end{array}$ \\
\hline Release area & $10 \mathrm{~m}^{2}$ & $\begin{array}{l}\text { Room: } 20 \mathrm{~m}^{3} ; \mathrm{I} \times \mathrm{w} \times \mathrm{h}=4 \times 2 \times 2.5 \mathrm{~m} \text {. The surface of the } \\
\text { wooden wall is } 4 \times 2.5=10 \mathrm{~m}^{2}\end{array}$ \\
\hline Mol. weight matrix & $300 \mathrm{~g} / \mathrm{mol}$ & $\begin{array}{l}\text { For solvent rich paint ( } 45 \% \text { solvent, } \mathrm{Mw} 140) \text {; the } \\
\text { compound of interest is not the main solvent }\end{array}$ \\
\hline \multicolumn{3}{|l|}{ Dermal, constant rate } \\
\hline Contact rate & $30 \mathrm{mg} / \mathrm{min}$ & $\begin{array}{l}\text { Default value for downward painting and painting } \\
\text { directed to the side; all paint products }\end{array}$ \\
\hline Release duration & $120 \mathrm{~min}$ & I.e. application duration \\
\hline \multicolumn{3}{|c|}{ Brush / roller painting, high solid paint ${ }^{\text {a) }}$} \\
\hline Frequency & 1 year $^{-1}$ & $\begin{array}{l}\text { Usage } 2 \text { times a year one or a few days after each other, } \\
\text { once per } 2 \text { years - based on two studies }\end{array}$ \\
\hline \multicolumn{3}{|c|}{ Inhalation, evaporation from increasing area } \\
\hline Exposure duration & $132 \mathrm{~min}$ & See "Brush / roller painting, solvent rich paint" \\
\hline Application duration & $120 \mathrm{~min}$ & Estimate \\
\hline Product amount & $1300 \mathrm{~g}$ & $\begin{array}{l}\text { For painting a wooden wall of } 10 \mathrm{~m}^{2}, 1 \text { liter or } 1300 \mathrm{~g} \text { paint } \\
\text { is required (paint coverage } 10 \mathrm{~m}^{2} / \mathrm{l} \text {, density } 1.3 \mathrm{~g} / \mathrm{cm}^{3} \text { ) }\end{array}$ \\
\hline Release area & $10 \mathrm{~m}^{2}$ & $\begin{array}{l}\text { Room: } 20 \mathrm{~m}^{3} ; \mathrm{I} \times \mathrm{w} \times \mathrm{h}=4 \times 2 \times 2.5 \mathrm{~m} \text {. The surface of the } \\
\text { wooden wall is } 4 \times 2.5=10 \mathrm{~m}^{2}\end{array}$ \\
\hline Mol weight matrix & $550 \mathrm{~g} / \mathrm{mol}$ & $\begin{array}{l}\text { Default value for high solid paint ( } 25 \% \text { solvent, } M w 140 \text { ); } \\
\text { the compound of interest is not the main solvent }\end{array}$ \\
\hline \multicolumn{3}{|l|}{ Dermal, constant rate } \\
\hline Contact rate & $30 \mathrm{mg} / \mathrm{min}$ & See "Brush / roller painting, solvent rich paint" \\
\hline Release duration & $120 \mathrm{~min}$ & I.e. application duration \\
\hline \multicolumn{3}{|c|}{ Brush / roller painting, waterborne paint ${ }^{a)}$} \\
\hline Frequency & 1 year $^{-1}$ & $\begin{array}{l}\text { Usage } 2 \text { times a year one or a few days after each other, } \\
\text { once per } 2 \text { years - based on two studies }\end{array}$ \\
\hline \multicolumn{3}{|c|}{ Inhalation, evaporation from increasing area } \\
\hline Exposure duration & $132 \mathrm{~min}$ & See "Brush / roller painting, solvent rich paint" \\
\hline Application duration & $120 \mathrm{~min}$ & Estimate \\
\hline Product amount & $1250 \mathrm{~g}$ & $\begin{array}{l}\text { For painting a wooden wall of } 10 \mathrm{~m}^{2}, 1 \text { liter or } 1250 \mathrm{~g} \\
\text { paint is necessary (paint coverage } 10 \mathrm{~m}^{2} / \mathrm{l} \text {, density } \\
1.25 \mathrm{mg} / \mathrm{cm}^{3} \text { ) }\end{array}$ \\
\hline Release area & $10 \mathrm{~m}^{2}$ & $\begin{array}{l}\text { Room: } 20 \mathrm{~m}^{3} ; 1 \times \mathrm{w} \times \mathrm{h}=4 \times 2 \times 2.5 \mathrm{~m} \text {. The surface of the } \\
\text { wooden wall is } 4 \times 2.5=10 \mathrm{~m}^{2}\end{array}$ \\
\hline Mol weight matrix & $45 \mathrm{~g} / \mathrm{mol}$ & $\begin{array}{l}\text { Default value for waterborne paint ( } 40 \% \text { water, } M w 18 \text { ); } \\
\text { the compound of interest is not the main solvent }\end{array}$ \\
\hline \multicolumn{3}{|l|}{ Dermal, constant rate } \\
\hline Contact rate & $30 \mathrm{mg} / \mathrm{min}$ & See "Brush / roller painting, solvent rich paint" \\
\hline Release duration & $120 \mathrm{~min}$ & I.e. application duration \\
\hline \multicolumn{3}{|c|}{ Brush / roller painting, waterborne wall paint ${ }^{a)}$} \\
\hline Frequency & 2 year $^{-1}$ & Estimate based on two studies \\
\hline \multicolumn{3}{|c|}{ Inhalation, evaporation from increasing area } \\
\hline Exposure duration & $132 \mathrm{~min}$ & See "Brush / roller painting, solvent rich paint" \\
\hline Application duration & $120 \mathrm{~min}$ & Estimate \\
\hline Product amount & $3750 \mathrm{~g}$ & $\begin{array}{l}\text { With } 1 \text { liter latex } 6 \mathrm{~m}^{2} \text { can be painted. For painting two } \\
\text { walls of in total } 15 \mathrm{~m}^{2}, 2.5 \text { liter or } 3.75 \mathrm{~kg} \text { paint is neces- } \\
\text { sary (density } 1.5 \mathrm{~g} / \mathrm{cm}^{3} \text { ) }\end{array}$ \\
\hline Mol. weight matrix & $120 \mathrm{~g} / \mathrm{mol}$ & $\begin{array}{l}\text { Default value for wall paint, waterborne ( } 15 \% \text { water, } \mathrm{Mw} \\
18) \text {; the compound of interest is not the main solvent }\end{array}$ \\
\hline \multicolumn{3}{|l|}{ Dermal, constant rate } \\
\hline Contact rate & $30 \mathrm{mg} / \mathrm{min}$ & See "Brush / roller painting, solvent rich paint" \\
\hline Release duration & $120 \mathrm{~min}$ & I.e. application duration \\
\hline
\end{tabular}




\begin{tabular}{|c|c|c|}
\hline & Default value & Comment \\
\hline \multicolumn{3}{|c|}{ Two-component paint: mixing and loading } \\
\hline Frequency & $1 / 3$ year $^{-1}$ & Estimate; once per three years \\
\hline \multicolumn{3}{|c|}{ Inhalation, evaporation constant release area } \\
\hline Exposure duration & $5 \mathrm{~min}$ & Estimate \\
\hline Application duration & $5 \mathrm{~min}$ & Estimate \\
\hline Product amount & NA & $\begin{array}{l}\text { The product amount, the quantity of paint which is } \\
\text { mixed and loaded, depends on the scenario which is }\end{array}$ \\
\hline Room volume & $1 \mathrm{~m}^{3}$ & $\begin{array}{l}\text { "Room volume" is interpreted here as "personal space": } \\
\text { a small volume of } 1 \mathrm{~m}^{3} \text { around the user. A small volume } \\
\text { of the user, for the short use duration in which the } \\
\text { treatment takes place }\end{array}$ \\
\hline Ventilation rate & $0.6 \mathrm{~h}^{-1}$ & $\begin{array}{l}\text { The ventilation rate for an unspecified room is used, } \\
\text { because no information is available on the ventilation } \\
\text { rate near the user. }\end{array}$ \\
\hline Release area & $95 \mathrm{~cm}^{2}$ & Estimate \\
\hline Mol. weight matrix & $3000 \mathrm{~g} / \mathrm{mol}$ & $\begin{array}{l}\text { Default value for paint where the compound of interest } \\
\text { is the main solvent }\end{array}$ \\
\hline \multicolumn{3}{|c|}{ Dermal, instant application } \\
\hline Product amount & $50 \mathrm{mg} /$ event & Based on different studies \\
\hline
\end{tabular}

a) The task is performed in a small, non-specified room of $20 \mathrm{~m}^{3}$ with a low ventilation rate $\left(0.6 \mathrm{~h}^{-1}\right)$.

Source: Modified from the Paint Products Fact Sheet, RIVM (2007b).

Table 11.9: Default Values for Spray Painting

\begin{tabular}{|c|c|c|}
\hline & Default value & Comment \\
\hline \multicolumn{3}{|c|}{ Spraying paint with a spray can } \\
\hline Frequency & 2 year $^{-1}$ & Estimate \\
\hline \multicolumn{3}{|l|}{ Inhalation, spray model } \\
\hline Spray duration & $15 \mathrm{~min}$ & Based on different studies \\
\hline Exposure duration & $20 \mathrm{~min}$ & $\begin{array}{l}\text { It is assumed that the garage is left } 5 \text { minutes after } \\
\text { spraying }\end{array}$ \\
\hline Mass generation rate & $0.33 \mathrm{~g} / \mathrm{sec}$ & $300 \mathrm{~g}$ of paint is sprayed in 15 minutes \\
\hline Airborne fraction & $1 \mathrm{~g} / \mathrm{g}$ & See Table 11.2.1.1. \\
\hline Weight fraction nonvolatile & 0.3 & Based on the composition of paint spray cans \\
\hline Density non-volatile & $1.5 \mathrm{~g} / \mathrm{cm}^{3}$ & Based on the composition - see Table 11.2.1.2. \\
\hline Initial droplet distribution & $30 \mu \mathrm{m}(0.8)$ & See "Cosmetics" \\
\hline \multicolumn{3}{|l|}{ - median (CV) } \\
\hline Inhalation cut-off diameter & $15 \mu \mathrm{m}$ & See "Cosmetics" \\
\hline \multicolumn{3}{|l|}{ Dermal, constant rate } \\
\hline Contact rate & $100 \mathrm{mg} / \mathrm{min}$ & See "Cleaning products - aerosol spray cans" \\
\hline Release duration & $15 \mathrm{~min}$ & I.e. spray duration \\
\hline \multicolumn{3}{|l|}{ Pneumatic spraying of paint } \\
\hline Frequency & 2 year $^{-1}$ & Estimate \\
\hline \multicolumn{3}{|l|}{ Inhalation, spray model } \\
\hline Spray duration & $800 \mathrm{sec}$ & $\begin{array}{l}\text { Spraying } 400 \mathrm{~g} \text { of paint (two radiators) with a mean mass } \\
\text { generation rate of } 0.5 \mathrm{~g} / \mathrm{sec} \text { lasts } 800 \mathrm{sec} \text { ( } 13.3 \text { minutes). }\end{array}$ \\
\hline Exposure duration & $25 \mathrm{~min}$ & $\begin{array}{l}\text { It is assumed that cleaning after spraying lasts circa } 10 \\
\text { minutes and that the garage is left afterwards }\end{array}$ \\
\hline Mass generation rate & $0.5 \mathrm{~g} / \mathrm{sec}$ & $\begin{array}{l}\text { It is assumed that the mean mass generation rate is } \\
50 \% \text { higher than for a spray can, i.e. } 0.5 \mathrm{~g} / \mathrm{sec} \text {. }\end{array}$ \\
\hline Airborne fraction & $0.2 \mathrm{~g} / \mathrm{g}$ & See Table 11.2.1.1. \\
\hline Weight fraction nonvolatile & 0.5 & Based on the composition \\
\hline Density non-volatile & $1.5 \mathrm{~g} / \mathrm{cm}^{3}$ & See Table 11.2.1.2. \\
\hline Initial droplet distribution & $50 \mu \mathrm{m}(0.6)$ & See "Cosmetics" \\
\hline \multicolumn{3}{|l|}{ - median (CV) } \\
\hline Inhalation cut-off diameter & $15 \mu \mathrm{m}$ & See "Cosmetics" \\
\hline \multicolumn{3}{|l|}{ Dermal, constant rate } \\
\hline Contact rate & $110 \mathrm{mg} / \mathrm{min}$ & Default value for pneumatic spraying - surface spraying \\
\hline Release duration & $800 \mathrm{sec}$ & I.e. spray duration \\
\hline
\end{tabular}

Both tasks are assumed to be performed in a garage which has a volume of $34 \mathrm{~m}^{3}$, is $2.25 \mathrm{~m}$ high and has a ventilation rate of $1.5 \mathrm{~h}^{-1}$.

Source: Modified from the Paint Products Fact Sheet, RIVM (2007b). 


\subsubsection{Disinfectant Products}

Disinfectant products are used to control or to prevent growth of microorganisms i.e. bacteria, viruses, and fungi. There is a great diversity in use and application types for the products. There are liquids, granulates, powders, tablets, gases. Some of these products can be used without any preparation, while others have to be processed (mixed and loaded) before use, for example by diluting or cutting up (RIVM, 2006d). In the Disinfectant Products Fact Sheet, RIVM (2006d), the "disinfectant products" are divided into the following product categories: algae, green deposit removers, swimming pool disinfectants, disinfectants for animal accommodations or animal transport vehicles (RIVM, 2006d). All models and parameters have been described earlier in the sections 11.2.111.2.4. Table 11.2.10 presents disinfectant products to be used by consumers. The standard room height is $2.5 \mathrm{~m}$, the uptake fraction is 1 for oral, dermal and inhalation exposure and the room temperature is $20^{\circ} \mathrm{C}$. It is assumed that exposure takes place during light exercise; therefore, the inhalation rate is set at 24.1 litre/min.

Table 11.10: Default Values for Disinfectant Products

\begin{tabular}{|c|c|c|}
\hline & Default Value & Comments \\
\hline \multicolumn{3}{|c|}{ Algae removers: mixing and loading liquid } \\
\hline Frequency & 3 year $^{-1}$ & $\begin{array}{l}\text { It is assumed that the user treats the pavement in the spring, } \\
\text { in the summer and before the winter. }\end{array}$ \\
\hline \multicolumn{3}{|l|}{ Dermal, instant application } \\
\hline Exposed area & $215 \mathrm{~cm}^{2}$ & One palm: $1 / 4$ area hands \\
\hline Product amount & $0.01 \mathrm{~g}$ & $\begin{array}{l}\text { Default value for mixing and loading of a liquid per } \\
\text { operation. Based on different studies. }\end{array}$ \\
\hline Exposure time & $3 \mathrm{~min}$ & $\begin{array}{l}\text { Default value for mixing and loading of a liquid. It is assumed } \\
\text { that the exposure time (i.e. the contact time of the formula- } \\
\text { tion with the skin) is longer than the application duration ( } 1.33 \\
\mathrm{~min} \text { ) and it is set at } 3 \text { minutes. }\end{array}$ \\
\hline \multicolumn{3}{|l|}{ Algae removers: spraying } \\
\hline $\begin{array}{l}\text { Frequency } \\
\text { Inhalation, spray model }\end{array}$ & 3 year $^{-1}$ & See "Algae removers: mixing and loading liquid" \\
\hline Spray duration & $16.7 \mathrm{~min}$ & $\begin{array}{l}\text { The spray duration can be calculated by dividing the volume } \\
\text { of diluted formulation by the mass generation rate i.e. } 5000 \\
\mathrm{ml} \text { divided by } 300 \mathrm{ml} / \mathrm{min} \text { resulting in } 16.7 \text { minutes, which is } \\
\text { set as default value for both spray and exposure duration }\end{array}$ \\
\hline Exposure duration & $16.7 \mathrm{~min}$ & I.e. spray duration \\
\hline Room volume & $62.5 \mathrm{~m}^{3}$ & $\begin{array}{l}\text { ConsExpo is developed for products applied indoors. As the } \\
\text { spraying scenario describes application outdoors, the } \\
\text { "spray" model will overestimate the inhalation exposure. } \\
\text { For the scenario, a "room" volume is defined as } 62.5 \mathrm{~m}^{3} \text {, i.e. } \\
25-\mathrm{m}^{2} \text { surface area multiplied with } 2.5 \mathrm{~m} \text { (standard room) } \\
\text { height. This is a worst-case situation! }\end{array}$ \\
\hline Ventilation rate & $0.6 \mathrm{hr}^{-1}$ & Unspecified room \\
\hline Mass generation rate & $5.0 \mathrm{~g} / \mathrm{s}$ & Estimate. Based on product information \\
\hline Airborne fraction & 0.2 & See Table 11.2.1.1. \\
\hline Weight fraction non-volatile & - & $\begin{array}{l}\text { Calculated. In trigger sprays, the weight fraction of the } \\
\text { non-volatile compounds is anyhow the weight fraction of } \\
\text { the active substance.. }\end{array}$ \\
\hline Density non-volatile & $1.8 \mathrm{~g} / \mathrm{cm}^{3}$ & $\begin{array}{l}\text { Default value for complex mixture of (especially organic) } \\
\text { compounds }\end{array}$ \\
\hline $\begin{array}{l}\text { Initial particle distribution } \\
\text { Median (CV) }\end{array}$ & $50 \mu \mathrm{m}(0.6)$ & Default value (worst-case) for disinfectant trigger sprays \\
\hline Inhalation cut-off diameter & $15 \mu \mathrm{m}$ & \\
\hline
\end{tabular}




\begin{tabular}{|c|c|c|}
\hline & Default Value & Comments \\
\hline \multicolumn{3}{|l|}{ Dermal, constant rate } \\
\hline Contact rate & $540 \mathrm{mg} / \mathrm{min}$ & $\begin{array}{l}\text { The default value for contact rate is } 32.35 \mathrm{~g} / \mathrm{hr} \text { (density } 1 \\
\mathrm{~g} / \mathrm{cm}^{3} \text { ) }\end{array}$ \\
\hline Release duration & $16.7 \mathrm{~min}$ & l.e. spray duration \\
\hline \multicolumn{3}{|c|}{ Algae removers: pouring and brushing } \\
\hline Frequency & 3 year $^{-1}$ & See "Algae removers: mixing and loading liquid" \\
\hline \multicolumn{3}{|c|}{ Dermal, instant application } \\
\hline Exposed area & $3300 \mathrm{~cm}^{2}$ & Area hands and arms \\
\hline Product amount dilution & $5 \mathrm{~g}$ & $\begin{array}{l}\text { During brushing the pavement, droplets of the solution } \\
\text { might settle on the skin. For estimating dermal exposure } \\
\text { due to droplets, } 0.05 \% \text { of the diluted formulation is taken } \\
\text { as default value for amount of spillage. An amount of } 10 \\
\text { litres dilution is required for brushing a pavement surface } \\
\text { area of } 25 \mathrm{~m}^{2} \text {; therefore, the dermal exposure is } 0.05 \% \text { of } \\
10000 \mathrm{ml} \text { i.e. } 5 \mathrm{~g} \text { diluted formulation (density } 1 \mathrm{~g} / \mathrm{cm}^{3} \text { ). }\end{array}$ \\
\hline Exposure time & $25 \mathrm{~min}$ & $\begin{array}{l}\text { The exposure time equals the application duration, which is } \\
\text { estimated at } 25 \text { minutes i.e. one minute for pouring and } \\
\text { brushing per square metre. }\end{array}$ \\
\hline \multicolumn{3}{|l|}{ Mould removers: spraying } \\
\hline Frequency & 1 year $^{-1}$ & $\begin{array}{l}\text { It is assumed that after the winter the user treats the } \\
\text { contaminated surface area i.e. once a year. }\end{array}$ \\
\hline
\end{tabular}

Both registered black mould removers have a content of $500 \mathrm{ml}$ ). Both directions for use state that the content is enough for a surface area of 2 to $3 \mathrm{~m}^{2}$. It is assumed that for $3 \mathrm{~m}^{2}$ an amount of $500 \mathrm{ml}$ (density $1 \mathrm{~g} / \mathrm{cm}^{3}$ ) is used. With a generation rate of $0.75 \mathrm{~g} / \mathrm{sec}$, a spray duration of 11.1 minutes is calculated.

$\begin{array}{ll}\text { Exposure duration } & 13 \mathrm{~min} \\ \text { Room volume } & 10 \mathrm{~m}^{3} \\ \text { Ventilation rate } & 2 \mathrm{hr}^{-1} \\ \text { Mass generation rate } & 0.75 \mathrm{~g} / \mathrm{s}\end{array}$

After spraying, the user leaves the bathroom

Bathroom

Bathroom

The default value for the mass generation rate is given as $1.5 \mathrm{~g}$ formulation/sec. It is assumed that the total duration of the application is twice as long as the actual spraying duration; therefore, the default value for the average mass generation rate is set at $0.75 \mathrm{~g} / \mathrm{s}$.

Airborne fraction

0.2

Weight fraction non-volatile See Table 11.2.1.1.

Calculated. In trigger sprays, the weight fraction of the non-volatile compounds is anyhow the weight fraction of the active substance.

Density non-volatile

Median (CV)

Inhalation cut-off diameter

$15 \mu \mathrm{m}$

Dermal, constant rate

Contact rate

$46 \mathrm{mg} / \mathrm{min}$

Release duration

See "Algae removers: spraying"

Mould removers: rinsing

Frequency

1 year $^{-1}$

I.e. spray duration

Dermal, instant application

Exposed area

$1900 \mathrm{~cm}^{2}$

See "Mould removers: spraying"

Product amount dilution

Skin surface area of forearms and hands

During rinsing the treated surface area, the user has

dermal contact with the diluted formulation. The default value for the thickness of film layer on skin is $0.01 \mathrm{~cm}$. With an exposed area of $1900 \mathrm{~cm}^{2}$ the default value for (diluted) amount is 19 grams

The amount of black mould remover on the surface is estimated at $85 \%$ of $500 \mathrm{~g}$ i.e. $425 \mathrm{~g}$ disinfectant product which is rinsed off. It is assumed that about 4.2 litres water is used to rinse the treated area; thus, the dilution is 10 times

Concentration, diffusion Density $1 \mathrm{~g} / \mathrm{cm}^{3}$

through skin

$13 \mathrm{~min}$

The default value for the cleaning duration is set at 4.35 $\mathrm{min} / \mathrm{m}^{2}$; this means that the exposure time for $3 \mathrm{~m}^{2}$ is 13 minutes. Based on different studies. 


\begin{tabular}{|c|c|c|}
\hline & Default Value & Comments \\
\hline \multicolumn{3}{|c|}{ Disinfectants for use indoors: spraying } \\
\hline \multicolumn{3}{|l|}{ Inhalation, spray model } \\
\hline Spray duration & $0.51 \mathrm{~min}$ & $\begin{array}{l}\text { In the instructions for use of the disinfectant was stated } \\
\text { that the surface must keep wet during the "leaving on" } \\
\text { time. Therefore, the maximum amount is taken and to } \\
\text { spray a kitchen working top of } 1.71 \mathrm{~m}^{2} \text { requires an } \\
\text { amount of } 22.87 \text { grams. Consequently, with a mass } \\
\text { generation rate of } 0.75 \mathrm{~g} / \mathrm{sec} \text {, the spray duration can be } \\
\text { calculated as } 0.51 \text { minutes. }\end{array}$ \\
\hline Exposure duration & $60 \mathrm{~min}$ & $\begin{array}{l}\text { The exposure duration is the sum of spraying time, time of } \\
\text { leaving on, the cleaning time and the time staying in the } \\
\text { specified room after application of the disinfectant products. }\end{array}$ \\
\hline Room volume & $15 \mathrm{~m}^{3}$ & Kitchen \\
\hline Ventilation rate & $2.5 \mathrm{hr}^{-1}$ & Kitchen \\
\hline Mass generation rate & $0.75 \mathrm{~g} / \mathrm{s}$ & $\begin{array}{l}\text { The default value for the mass generation rate is given as } \\
1.5 \mathrm{~g} \text { formulation } / \mathrm{sec} \text {. It is assumed that the total duration } \\
\text { of the application is twice as long as the actual spraying } \\
\text { duration; therefore, the default value for the average mass } \\
\text { generation rate is set at } 0.75 \mathrm{~g} / \mathrm{s} \text {. }\end{array}$ \\
\hline Airborne fraction & 0.2 & See Table 11.2.1.1. \\
\hline Weight fraction non-volatile & - & $\begin{array}{l}\text { Calculated. In trigger sprays, the weight fraction of the } \\
\text { non-volatile compounds is anyhow the weight fraction of } \\
\text { the active substance. }\end{array}$ \\
\hline Density non-volatile & $1.8 \mathrm{~g} / \mathrm{cm}^{3}$ & See "Algae removers: spraying" \\
\hline $\begin{array}{l}\text { Initial particle distribution } \\
\text { Median (CV) }\end{array}$ & $50 \mu \mathrm{m}(0.6)$ & See "Algae removers: spraying" \\
\hline $\begin{array}{l}\text { Inhalation cut-off diameter } \\
\text { Dermal, constant rate }\end{array}$ & $15 \mu \mathrm{m}$ & Default value \\
\hline Contact rate & $46 \mathrm{mg} / \mathrm{min}$ & See "Algae removers: spraying" \\
\hline Release duration & $0.51 \mathrm{~min}$ & I.e. spray duration \\
\hline \multicolumn{3}{|l|}{ Surface disinfectants: wiping } \\
\hline Frequency & 365 year $^{-1}$ & Estimate: 1 day $^{-1}$ \\
\hline \multicolumn{3}{|l|}{ Dermal, instant application } \\
\hline Exposed area & $215 \mathrm{~cm}^{2}$ & One palm; $1 / 4$ area hands \\
\hline Product amount & $0.02 \mathrm{~g}$ & $\begin{array}{l}\text { The worst-case estimate is that } 0.1 \% \text { of the amount on } \\
\text { the surface area (i.e. } 19.4 \mathrm{~g} \text { ) contacts the skin }\end{array}$ \\
\hline Exposure time & $3 \mathrm{~min}$ & $\begin{array}{l}\text { Estimate based on one study and the area of a kitchen } \\
\text { work top }\left(1.71 \mathrm{~m}^{2}\right)\end{array}$ \\
\hline \multicolumn{3}{|c|}{ Algae control liquids: mixing and loading liquid } \\
\hline Frequency & 28 year $^{-1}$ & $\begin{array}{l}\text { For the maintenance ration, the dosage is every } 5 \text { days for } \\
\text { a period of } 4 \text { months. It is assumed that before, during } \\
\text { and after the winter period i.e. during the off-season, a } \\
\text { higher dose is applied (see use). }\end{array}$ \\
\hline \multicolumn{3}{|l|}{ Dermal, instant application } \\
\hline Exposed area & $215 \mathrm{~cm}^{2}$ & One palm: $1 / 4$ area hands \\
\hline Product amount & $0.01 \mathrm{~g}$ & See "Algae removers: mixing and loading liquid" \\
\hline Exposure time & $3 \mathrm{~min}$ & See "Algae removers: mixing and loading liquid" \\
\hline \multicolumn{3}{|c|}{ Application algae control liquid, swimming pool } \\
\hline Frequency & 28 year $^{-1}$ & See "Algae control liquids: mixing and loading liquid" \\
\hline \multicolumn{3}{|l|}{ Dermal, instant application } \\
\hline Exposed area & $1900 \mathrm{~cm}^{2}$ & Area hands and forearms \\
\hline Product amount dilution & $0.5 \mathrm{~g}$ & $\begin{array}{l}\text { The contamination of emptying a } 10 \mathrm{~L} \text { container is } 0.50 \mathrm{ml} \\
\text { formulation per operation. In this case, the contamination } \\
\text { is set at } 0.5 \mathrm{~g} \text { of diluted formulation (density } 1 \mathrm{~g} / \mathrm{cm}^{3} \text { ) } \\
\text { during emptying the bucket }\end{array}$ \\
\hline Weight fraction dilution & $W_{f} / 10$ & $\begin{array}{l}\text { The dilution is set at } 10 \text { times and so, the weight fraction } \\
\text { should be divided by } 10 \text {. }\end{array}$ \\
\hline $\begin{array}{l}\text { Concentration, diffusion } \\
\text { through skin }\end{array}$ & $W_{f} / 10 \mathrm{~g} / \mathrm{cm}^{3}$ & Density $1 \mathrm{~g} / \mathrm{cm}^{3}$ \\
\hline Exposure time & $5 \mathrm{~min}$ & Estimate \\
\hline
\end{tabular}




\begin{tabular}{|c|c|c|}
\hline & Default Value & Comments \\
\hline \multicolumn{3}{|c|}{ Dissolving granules: mixing and loading } \\
\hline Frequency & 122 year $^{-1}$ & Once a day for a period of 4 months \\
\hline \multicolumn{3}{|l|}{ Dermal, constant rate } \\
\hline Contact rate & $0.033 \mathrm{mg} / \mathrm{min}$ & $\begin{array}{l}\text { The dermal exposure for consumers is estimated at } 2 \\
\mathrm{mg} / \mathrm{hr}\end{array}$ \\
\hline Release duration & $1.33 \mathrm{~min}$ & $\begin{array}{l}\text { It is assumed that the release duration has the same value } \\
\text { as for application duration during mixing and loading } \\
\text { liquids i.e. } 1.33 \text { minutes. }\end{array}$ \\
\hline \multicolumn{3}{|c|}{ Application granules, swimming pool } \\
\hline Frequency & 122 year $^{-1}$ & Once a day for a period of 4 months \\
\hline \multicolumn{3}{|l|}{ Dermal, instant application } \\
\hline Exposed area & $1900 \mathrm{~cm}^{2}$ & Area hands and forearms \\
\hline Product amount dilution & $0.5 \mathrm{~g}$ & See "Application algae control liquid, swimming pool" \\
\hline Weight fraction dilution & $W_{f} / 20$ & Estimate \\
\hline $\begin{array}{l}\text { Concentration, diffusion } \\
\text { through skin }\end{array}$ & $W_{f} / 20$ & Density water $1 \mathrm{~g} / \mathrm{cm}^{3}$ \\
\hline Exposure time & $5 \mathrm{~min}$ & Estimate \\
\hline \multicolumn{3}{|c|}{ Dissolving tablets: mixing and loading } \\
\hline Frequency & 122 year $^{-1}$ & Ten tablets of $20 \mathrm{~g}$ is used every days during 4 months \\
\hline \multicolumn{3}{|l|}{ Dermal, constant rate } \\
\hline Contact rate & $0.033 \mathrm{mg} / \mathrm{min}$ & See "Dissolving granules: mixing and loading" \\
\hline Release duration & $1 \mathrm{~min}$ & $\begin{array}{l}10 \text { tablets. The release duration is the time during which the } \\
\text { compound is applied. For loading tablets, the release duration } \\
\text { is estimated at } 6 \text { seconds per tablet }\end{array}$ \\
\hline \multicolumn{3}{|c|}{ Application tablets, swimming pool } \\
\hline Frequency & 122 year-1 & Ten tablets of $20 \mathrm{~g}$ is used every days during 4 months \\
\hline \multicolumn{3}{|l|}{ Dermal, instant application } \\
\hline Exposed area & $1900 \mathrm{~cm}^{2}$ & Area hands and forearms \\
\hline Product amount dilution & $0.5 \mathrm{~g}$ & See "Application algae control liquid, swimming pool" \\
\hline Weight fraction dilution & $W_{f} / 20$ & Estimate \\
\hline $\begin{array}{l}\text { Concentration, diffusion } \\
\text { through skin }\end{array}$ & $W_{f} / 20$ & Density water $1 \mathrm{~g} / \mathrm{cm}^{3}$ \\
\hline Exposure time & $5 \mathrm{~min}$ & Estimate \\
\hline \multicolumn{3}{|c|}{ Post-application swimming pool disinfectants; outdoor private swimming pool } \\
\hline Frequency & 52 year $^{-1}$ & $\begin{array}{l}\text { During a period of } 4 \text { months the user swims } 3 \text { times a } \\
\text { week }\end{array}$ \\
\hline \multicolumn{3}{|l|}{ Dermal, instant application } \\
\hline Exposed area & $17500 \mathrm{~cm}^{2}$ & The body \\
\hline Product amount dilution & $17500 \mathrm{~g}$ & $\begin{array}{l}\text { It is assumed that not the total amount of swim water is in } \\
\text { contact with the skin but only a layer of } 1 \mathrm{~cm} \text { around the } \\
\text { exposed skin; thus, the amount of swim water is } 17500 \mathrm{~cm}^{3} \text {. }\end{array}$ \\
\hline Exposure time & $60 \mathrm{~min}$ & Estimate \\
\hline \multicolumn{3}{|l|}{ Oral, constant rate } \\
\hline Ingestion rate & $830 \mathrm{mg} / \mathrm{min}$ & $\begin{array}{l}\text { The default value for ingestion rate is set at } 50 \mathrm{ml} / \mathrm{hr} \text { or } \\
830 \mathrm{mg} / \mathrm{min} \text { (density } 1 \mathrm{~g} / \mathrm{cm}^{3} \text { ). Based on different studies }\end{array}$ \\
\hline Exposure time & $60 \mathrm{~min}$ & Estimate \\
\hline \multicolumn{3}{|c|}{ Waterbed conditioners: mixing and loading liquid } \\
\hline Frequency & 2 year $^{-1}$ & Estimate \\
\hline \multicolumn{3}{|l|}{ Dermal, instant application } \\
\hline Exposed area & $215 \mathrm{~cm}^{2}$ & One palm: $1 / 4$ area hands \\
\hline Product amount & $0.01 \mathrm{~g}$ & See "Algae removers: mixing and loading liquid" \\
\hline Exposure time & $3 \mathrm{~min}$ & See "Algae removers: mixing and loading liquid" \\
\hline \multicolumn{3}{|c|}{ Disinfectants chemical toilets: mixing and loading liquid } \\
\hline Frequency & 7 year $^{-1}$ & $\begin{array}{l}\text { During a period of } 28 \text { days, the user empties the waste } \\
\text { tank every } 4 \text { days }\end{array}$ \\
\hline \multicolumn{3}{|c|}{ Inhalation, evaporation from a constant surface } \\
\hline Exposure duration & $1.33 \mathrm{~min}$ & Default value for mixing and loading liquid \\
\hline Product amount & $500 \mathrm{~g}$ & See "Algae removers: mixing and loading liquid" \\
\hline Room volume & $1 \mathrm{~m}^{3}$ & $\begin{array}{l}\text { "Room volume" is interpreted here as "personal volume": } \\
\text { a small area of } 1 \mathrm{~m}^{3} \text { around the user. }\end{array}$ \\
\hline Ventilation rate & $0.6 \mathrm{hr}^{-1}$ & Unspecified room \\
\hline Release area & $0.002 \mathrm{~m}^{2}$ & $\begin{array}{l}\text { It is assumed that evaporation takes place from a bottle } \\
\text { with a not-too-small circular opening with a } 5-\mathrm{cm} \text {. diame- } \\
\text { ter which gives a release areaof } 20 \mathrm{~cm}^{2}\end{array}$ \\
\hline Application duration & $1.33 \mathrm{~min}$ & See "exposure duration" \\
\hline Density & $1.0 \mathrm{~g} / \mathrm{cm}^{3}$ & Density water \\
\hline
\end{tabular}




\begin{tabular}{|c|c|c|}
\hline & Default Value & Comments \\
\hline \multicolumn{3}{|l|}{ Dermal, instant application } \\
\hline Exposed area & $215 \mathrm{~cm}^{2}$ & One palm: $1 / 4$ area hands \\
\hline Product amount & $0.01 \mathrm{~g}$ & See "Algae removers: mixing and loading liquid" \\
\hline Exposure time & $3 \mathrm{~min}$ & See "Algae removers: mixing and loading liquid" \\
\hline \multicolumn{3}{|c|}{ Disinfectants rubbish bins: mixing and loading liquid } \\
\hline Frequency & 6 year $^{-1}$ & $\begin{array}{l}\text { It is assumed that the user disinfects the rubbish bin every } \\
\text { month during springtime and summertime, which results in } \\
\text { a frequency of } 6 \text { times a year }\end{array}$ \\
\hline \multicolumn{3}{|l|}{ Dermal, instant application } \\
\hline Exposed area & $215 \mathrm{~cm}^{2}$ & One palm: $1 / 4$ area hands \\
\hline Product amount & $0.01 \mathrm{~g}$ & See "Algae removers: mixing and loading liquid" \\
\hline Exposure time & $3 \min$ & See "Algae removers: mixing and loading liquid" \\
\hline \multicolumn{3}{|c|}{ Disinfectants rubbish bins: application } \\
\hline Frequency & 6 year $^{-1}$ & See "Disinfectants rubbish bins: mixing and loading liquid" \\
\hline \multicolumn{3}{|l|}{ Dermal, instant application } \\
\hline Exposed area & $1900 \mathrm{~cm}^{2}$ & Area hands and forearms \\
\hline Product amount dilution & $5 \mathrm{~g}$ & $\begin{array}{l}\text { For estimating the dermal exposure, a percentage of } 0.1 \text { is } \\
\text { taken as default value for amount of spillage. An amount } \\
\text { of } 5 \text { litres solution is required for disinfecting a dustbin; } \\
\text { the dermal exposure is therefore } 5 \mathrm{ml} \text { i.e. } 5 \mathrm{~g} \text { diluted } \\
\text { formulation (density } 1 \mathrm{~g} / \mathrm{cm}^{3} \text { ). }\end{array}$ \\
\hline Exposure time & $5 \mathrm{~min}$ & $\begin{array}{l}\text { The exposure time is total duration for application, i.e. spread- } \\
\text { ing the diluted formulation and draining off the excess of } \\
\text { water, which is estimated at } 5 \text { minutes. }\end{array}$ \\
\hline \multicolumn{3}{|c|}{ Disinfectants bird accommodations: fumigation } \\
\hline Frequency & 12 year $^{-1}$ & $\begin{array}{l}\text { The use frequency mentioned by pigeon fanciers on the } \\
\text { internet were every fortnight, every month, twice a } \\
\text { quarter, and every quarter. The frequency is set at once a } \\
\text { month. }\end{array}$ \\
\hline \multicolumn{3}{|l|}{ Inhalation, constant rate } \\
\hline Exposure duration & $0.5 \mathrm{~min}$ & $\begin{array}{l}\text { It is assumed that the user leaves the dovecot directly } \\
\text { after lightning the tablet. The default value for exposure } \\
\text { duration for inhalation is set at } 0.5 \text { minute. At re-entry it is } \\
\text { assumed that the air concentration of active substance is } \\
\text { considerably diminished compared to the air concentra- } \\
\text { tion in the beginning of the disinfection. }\end{array}$ \\
\hline Product amount & $42.5 \mathrm{~g}$ & $\begin{array}{l}\text { One package of } 170 \text { grams (one tablet) is sufficient for a } \\
\text { room volume of } 75 \text { to } 100 \mathrm{~m}^{3} \text { that is } 1 / 4 \text { of tablet is suffi- } \\
\text { cient for } 17.5-25 \mathrm{~m}^{3} \text { (directions for use). Consequently, } \\
42.5 \text { grams of product will be applied for a dovecot of } 20 \\
\mathrm{~m}^{3} \text {. }\end{array}$ \\
\hline Room volume & $20 \mathrm{~m}^{3}$ & $\begin{array}{l}\text { The scenario describes a private user who sets fire to a } \\
\text { quarter of a tablet (about } 40 \text { grams) containing paraform- } \\
\text { aldehyde in a dovecot of } 20 \mathrm{~m}^{3} \text { measuring } 4.30 \mathrm{~m} \text { (length) } \\
\times 2.50 \mathrm{~m} \text { (height) } \times 1.85 \mathrm{~m} \text { (depth). }\end{array}$ \\
\hline Ventilation rate & $0.6 \mathrm{hr}^{-1}$ & Unspecified room \\
\hline Emission duration & $30 \mathrm{~min}$ & $\begin{array}{l}\text { It is assumed that it takes } 30 \text { minutes to burn up the } \\
\text { tablet with paraformaldehyde }\end{array}$ \\
\hline \multicolumn{3}{|l|}{ Dermal, constant rate } \\
\hline Contact rate & $0.033 \mathrm{mg} / \mathrm{min}$ & See "Dissolving granules: mixing and loading" \\
\hline Release duration & $0.5 \mathrm{~min}$ & $\begin{array}{l}\text { The default value for the release duration for mixing and } \\
\text { loading tablets is set at } 0.1 \text { minute. In this case, the user } \\
\text { has to break and lighten the tablet; the release duration is } \\
\text { estimated at } 0.5 \text { minute. }\end{array}$ \\
\hline \multicolumn{3}{|c|}{ Disinfectants animal accommodations: mixing and loading powder } \\
\hline Frequency & 2 year $^{-1}$ & Estimate \\
\hline \multicolumn{3}{|l|}{ Dermal, constant rate } \\
\hline Contact rate & $0.033 \mathrm{mg} / \mathrm{min}$ & See "Dissolving granules: mixing and loading" \\
\hline Release duration & $1.33 \mathrm{~min}$ & See "Dissolving granules: mixing and loading" \\
\hline
\end{tabular}




\begin{tabular}{|c|c|c|}
\hline & Default Value & Comments \\
\hline \multicolumn{3}{|c|}{ Disinfectants animal accommodations: mixing and loading tablets } \\
\hline Frequency & 2 year $^{-1}$ & Estimate \\
\hline \multicolumn{3}{|c|}{ Dermal, constant rate } \\
\hline Contact rate & $0.033 \mathrm{mg} / \mathrm{min}$ & See "Dissolving granules: mixing and loading" \\
\hline Release duration & $0.5 \mathrm{~min}$ & $\begin{array}{l}\text { The release duration is the time during which the compound is } \\
\text { applied. According to different instructions for use, } 4,5 \text { or } 10 \\
\text { tablets in } 10 \text { litres should be used for disinfection animal } \\
\text { accommodations; for tablets, the release duration is } 0.1 \\
\text { minute per tablet. It is assumed that } 5 \text { tablets are dissolved in } \\
5 \text { litres of water and the release duration is set at } 0.5 \text { min. }\end{array}$ \\
\hline
\end{tabular}

Disinfectants animal accommodations: wiping

$\begin{array}{ll}\text { Frequency } & 2 \text { year }^{-1} \\ \text { Dermal, instant application } & \\ \text { Exposed area } & 1900 \mathrm{~cm}^{2} \\ \text { Product amount dilution } & 19 \mathrm{~g} \\ \text { Exposure time } & 20 \mathrm{~min}\end{array}$

Estimate

Area hands \& forearms

See "Mould removers: rinsing"

The treated surface area is set at $20 \mathrm{~m}^{2}$. The application

"wiping" is more a question of spreading the diluted

formulation to disinfect the surface than of cleaning. As

default for wiping one minute per square meter is taken which results in an exposure duration of $20 \mathrm{~min}$.

Disinfectants animal transports: mixing and loading powder

$\begin{array}{lll}\begin{array}{l}\text { Frequency } \\ \text { Dermal, constant rate }\end{array} & 6 \text { year }^{-1} & \text { Estimate } \\ \begin{array}{ll}\text { Contact rate } \\ \text { Release duration }\end{array} & 0.033 \mathrm{mg} / \mathrm{min} & \text { See "Dissolving granules: mixing and loading" } \\ 1.33 \mathrm{~min} & \text { See "Dissolving granules: mixing and loading" }\end{array}$

Disinfectants animal transports: mixing and loading tablets

Frequency 6 year $^{-1} \quad$ Estimate

Dermal, constant rate

Contact rate $\quad 0.033 \mathrm{mg} / \mathrm{min}$

Release duration $\quad 1.0 \mathrm{~min}$

See "Dissolving granules: mixing and loading"

The release duration is the time during which the compound is applied. According to the use instruction, 10 tablets in 10 litres should be used for disinfection animal accommodations. For tablets, the release duration is 0.1 minute per tablet. When dissolving 10 tablets in 10 litres water, the total release duration will be 1.0 minute.

$\begin{aligned} & \text { Disinfectants animal transports: spraying } \\ & \text { Frequency } \\ & \text { Inhalation, spray model } \\ & \text { Spray duration }\end{aligned}$
$\begin{aligned} & \text {-1 } \\ & \text { Exposure duration }\end{aligned}$
Room volume

Estimate

The default value for exposure duration equals the duration for spraying which is set at 17 minutes i.e. $5000 \mathrm{ml}$ diluted formulation divided by $300 \mathrm{ml} / \mathrm{min}(5 \mathrm{~g} / \mathrm{sec})$

Room volume

$5 \mathrm{~m}^{3}$

$\begin{array}{ll}\text { Ventilation } & 0.6 \mathrm{hr}^{-1} \\ \text { Mass generation rate } & 5 \mathrm{~g} / \mathrm{s} \\ \text { Airborne fraction } & 0.2 \\ \text { Weight fraction non-volatile } & - \\ & \\ & 1.8 \mathrm{~g} / \mathrm{cm}^{3} \\ \begin{array}{l}\text { Density non-volatile } \\ \text { Initial particle distribution }\end{array} & 50 \mu \mathrm{m} \mathrm{(0.6)} \\ \begin{array}{l}\text { Median (CV) } \\ \text { Inhalation cut-off diameter }\end{array} & 15 \mu \mathrm{m} \\ \begin{array}{l}\text { Dermal, constant rate } \\ \text { Contact rate }\end{array} & 540 \mathrm{mg} / \mathrm{min} \\ \text { Release duration } & 17 \mathrm{~min}\end{array}$

As the spraying scenario describes application outdoors, the "spray" model will overestimate the inhalation exposure. For the scenario, a "room" volume is defined as $50 \mathrm{~m}^{3}$, i.e. $20-\mathrm{m}^{2}$ surface area (i.e. area around the trailer) multiplied with $2.5 \mathrm{~m}$ (standard room) height Unspecified room

Default vale

See Table 11.2.1.1.

Calculated. In trigger sprays, the weight fraction of the non-volatile compounds is anyhow the weight fraction of the active substance.

See "Algae removers: spraying"

See "Algae removers: spraying"

Default value

See "Algae removers: spraying"

I.e. spray duration 


\begin{tabular}{|c|c|c|}
\hline & Default Value & Comments \\
\hline \multicolumn{3}{|c|}{ Disinfectants milking machines: mixing and loading liquid } \\
\hline Frequency & 730 year $^{-1}$ & $\begin{array}{l}\text { The system cleaning of the automatic min-milking ma- } \\
\text { chine is done two times a day }\end{array}$ \\
\hline \multicolumn{3}{|c|}{ Dermal, instant application } \\
\hline Exposed area & $215 \mathrm{~cm}^{2}$ & One palm: $1 / 4$ area hands \\
\hline Product amount & $0.01 \mathrm{~g}$ & See "Algae removers: mixing and loading liquid" \\
\hline Exposure time & $3 \mathrm{~min}$ & See "Algae removers: mixing and loading liquid" \\
\hline \multicolumn{3}{|c|}{ Disinfectants milking machines: mixing and loading powder } \\
\hline Frequency & 730 year $^{-1}$ & $\begin{array}{l}\text { See "Disinfectants milking machines: mixing and loading } \\
\text { liquid" }\end{array}$ \\
\hline \multicolumn{3}{|c|}{ Dermal, constant rate } \\
\hline Contact rate & $0.033 \mathrm{mg} / \mathrm{min}$ & See "Dissolving granules: mixing and loading" \\
\hline Release duration & $1.33 \mathrm{~min}$ & See "Dissolving granules: mixing and loading" \\
\hline
\end{tabular}

Disinfectants milking machines: mixing and loading tablets

\begin{tabular}{|c|c|c|}
\hline Frequency & 730 year $^{-1}$ & $\begin{array}{l}\text { See "Disinfectants milking machines: mixing and loading } \\
\text { liquid" }\end{array}$ \\
\hline \multicolumn{3}{|l|}{ Dermal, constant rate } \\
\hline Contact rate & $0.033 \mathrm{mg} / \mathrm{min}$ & See "Dissolving granules: mixing and loading" \\
\hline Release duration & $0.1 \mathrm{~min}$ & $\begin{array}{l}\text { The release duration is the time during which the com- } \\
\text { pound is applied. According to the use instruction, one } \\
\text { tablet in } 10 \text { ( } 8 \text { ) litres should be used for disinfection } \\
\text { milking equipment. For tablets, the release duration is } 0.1 \\
\text { minute per tablet }\end{array}$ \\
\hline \multicolumn{3}{|c|}{ Disinfectants milking machines: disinfecting peripherals } \\
\hline Frequency & 730 year $^{-1}$ & $\begin{array}{l}\text { The system cleaning of the automatic min-milking ma- } \\
\text { chine is done two times a day }\end{array}$ \\
\hline \multicolumn{3}{|l|}{ Dermal, instant application } \\
\hline Exposed area & $1900 \mathrm{~cm}^{2}$ & Area hands and forearms \\
\hline Weight fraction dilution & - & $\begin{array}{l}\text { Calculated. In trigger sprays, the weight fraction of the } \\
\text { non-volatile compounds is anyhow the weight fraction of } \\
\text { the active substance. }\end{array}$ \\
\hline Product dilution & $19 \mathrm{~g}$ & Default value \\
\hline Exposure time & $5 \mathrm{~min}$ & Estimate \\
\hline
\end{tabular}

Post-application disinfectants drinking water: ingestion

\begin{tabular}{|c|c|c|}
\hline \multicolumn{3}{|c|}{ Post-application disinfectants drinking water: ingestion } \\
\hline Frequency & 14 year $^{-1}$ & $\begin{array}{l}\text { The scenario describes a private user who treats drinking } \\
\text { water during a fortnight while staying on locations with } \\
\text { bacteriological unreliable drinking water. The user drinks } \\
\text { every day } 2 \text { litres of water }\end{array}$ \\
\hline \multicolumn{3}{|l|}{ Oral, direct intake } \\
\hline Amount ingested dilution & $2000 \mathrm{~g}$ & See "frequency" \\
\hline Weight fraction dilution & - & $\begin{array}{l}\text { The weight fraction is the calculated weight fraction of the } \\
\text { diluted formulation }\end{array}$ \\
\hline \multicolumn{3}{|c|}{ Disinfectants water coolers: spraying } \\
\hline Frequency & 4 year $^{-1}$ & Estimate; every three months \\
\hline \multicolumn{3}{|l|}{ Inhalation, spray model } \\
\hline Spray duration & $0.9 \min$ & $\begin{array}{l}\text { With a mass generation rate of } 0.36 \mathrm{~g} / \mathrm{s} \text { and an amount of } \\
20 \mathrm{ml} \text { per application, the spray duration can be calculat- } \\
\text { ed as } 0.9 \mathrm{~min} \text { (density } 1 \mathrm{~g} / \mathrm{cm}^{3} \text { ). }\end{array}$ \\
\hline Exposure duration & $240 \mathrm{~min}$ & Estimate; four hours \\
\hline Room volume & $20 \mathrm{~m}^{3}$ & Unspecified room \\
\hline Ventilation rate & $0.6 \mathrm{hr}^{-1}$ & Unspecified room \\
\hline Mass generation rate & $0.36 \mathrm{~g} / \mathrm{s}$ & $\begin{array}{l}\text { It is assumed that the mass generation rate of the disin- } \\
\text { fectant spray is much smaller compared to trigger sprays } \\
(1.5 \mathrm{~g} / \mathrm{sec}) \text { and twice as large compared to eau de toilette } \\
\text { pump sprays. The default value for mass generation rate } \\
\text { is set at } 0.36 \mathrm{~g} / \mathrm{sec} \text {. }\end{array}$ \\
\hline Airborne fraction & 0.2 & See Table 11.2.1.1. \\
\hline Weight fraction non-volatile & - & $\begin{array}{l}\text { Calculated. In trigger sprays, the weight fraction of the } \\
\text { non-volatile compounds is anyhow the weight fraction of } \\
\text { the active substance. }\end{array}$ \\
\hline Density non-volatile & $1.8 \mathrm{~g} / \mathrm{cm}^{3}$ & See "Algae removers: spraying" \\
\hline Inhalation cut-off diameter & $15 \mu \mathrm{m}$ & Default value \\
\hline
\end{tabular}




\begin{tabular}{|c|c|c|}
\hline & Default Value & Comments \\
\hline \multicolumn{3}{|l|}{ Dermal, constant rate } \\
\hline Contact rate & $46 \mathrm{mg} / \mathrm{min}$ & See "Algae removers: spraying" \\
\hline Release duration & $0.9 \min$ & I.e. spray duration \\
\hline \multicolumn{3}{|c|}{ Post-application disinfectants water coolers: ingestion } \\
\hline Frequency & 4 year $^{-1}$ & \\
\hline \multicolumn{3}{|l|}{ Oral, direct intake } \\
\hline Amount ingested dilution & $1000 \mathrm{~g}$ & Estimate; one litre of water \\
\hline Weight fraction dilution & - & $\begin{array}{l}\text { The weight fraction is the calculated weight fraction of the } \\
\text { diluted formulation }\end{array}$ \\
\hline
\end{tabular}

Source: Modified from the Disinfectant Products Fact Sheets, RIVM (2006d).

\subsubsection{Children's toys}

In the Children's Toys Fact Sheet, RIVM (2002), default values on different toys are classified according to the ways in which children can be exposed instead of by product type.

Default values on mouthing times for children are calculated based on an extensive study by Groot et al. (cited in RIVM, 2002). Results from the study are multiplied by a factor of 1.5 to include the night-time because only daily duration was considered in the study. For mouthing times on a pacifier, only average times are known and no standard deviations. The calculated default values on mouthing times for children are presented in Table 11.11.

With regard to the age category, RIVM has in each case chosen the age category for which the highest exposure is expected. In practice, it means that the youngest children who played with a certain type of toy were chosen for the defaults. The youngest children are the lightest and have the highest uptake per kg of body weight for the same exposure (RIVM, 2002). Moreover, when applying default values, it is not possible to work with time periods, but instead, a point in time must be chosen. For example, a body weight to a child of 3 to 6 months cannot be attributed; you must choose a point in time (4.5 months) (RIVM, 2002).

\begin{tabular}{lrrrrr}
\multicolumn{2}{l}{ Table 11.11: Default Values on Mouthing Times for Children } \\
\hline Period $^{\text {a) }}$ (months) & Age $^{\text {a) }}$ (months) & \multicolumn{4}{c}{ Default mouthing times (min/day) } \\
\cline { 3 - 6 } & & Pacifier & Toys for mouthing $^{\text {b) }}$ & Other toys $^{\text {c) }}$ & Non toys $^{\text {d) }}$ \\
\hline $3-6$ & 4.5 & 285 & 11 & 27 & 8 \\
$6-12$ & 7.5 & 82 & 21 & 63 & 23 \\
$12-18$ & 13.5 & 52 & 0 & 9 & 26 \\
$18-36$ & 18 & 62 & 0 & 3 & 6
\end{tabular}

a) When applying default values, it is not possible to work with time periods. For example, you cannot attribute a body weight to a child of 3 to 6 months; you must choose a point in time ( 4.5 months).

b) Toys meant for mouthing: all kinds of teething rings, some rattles.

c) Other toys: cloth books, plastic books, cuddly toy.

d) Non toys: a piece of cloth, a piece of paper, a book for adults, flatware.

Reference: Modified from the Children's Toys Fact Sheet, RIVM (2002). 
If a child puts an object in its mouth, pieces of the object can break away, and these can be swallowed. When toys are mouthed, pieces can also break off and be swallowed, such as paint from the metal toy car, for example. Apart from putting objects in the mouth, substances can be taken in by hand-mouth contact.

In order to estimate default values on direct ingestion, the following parameters must be taken in to account: the composition of the ingested product, the density of the product, the dilution of the product before it is ingested and the amount of product taken in. However, due to lack of measurements data on the amount ingested, only estimates on a few specific toys are made: modelling clay, paint from a toy car, and a ball pen. When calculating default values on mouth-hand contact the composition of the product ingested and the ingestion rate (in volume-units per unit time) are the only parameters that has to be estimated (RIVM, 2002). Table 11.12 presents default values on direct ingestion of children's toys and Table 11.13 presents default values on hand-mouth contact.

Table 11.12: Default Values on Direct Ingestion of Children's Toys

\begin{tabular}{|c|c|c|}
\hline & Default value & Comments \\
\hline \multicolumn{3}{|l|}{ Modelling clay ${ }^{\mathrm{a})}$} \\
\hline \multicolumn{3}{|l|}{ Contact } \\
\hline Frequency & 52/year & Estimation \\
\hline Total duration & $60 \mathrm{~min}$ & Estimation \\
\hline Use duration & $60 \mathrm{~min}$ & Estimation \\
\hline \multicolumn{3}{|l|}{ Oral } \\
\hline Product volume & $0.5 \mathrm{~cm}^{3}$ & Estimation \\
\hline Density & $2 \mathrm{~g} / \mathrm{cm}^{3}$ & Estimation \\
\hline Absorbed fraction & 1 & Potential dose \\
\hline \multicolumn{3}{|c|}{ Paint from a toy car $^{\text {b) }}$} \\
\hline \multicolumn{3}{|l|}{ Contact } \\
\hline Frequency & 150/year & Estimation, 3x/week \\
\hline Total duration & $3 \min$ & See Table 3.2.1. "other toys" \\
\hline Use duration & $3 \min$ & See Table 3.2.1. "other toys" \\
\hline \multicolumn{3}{|l|}{ Oral } \\
\hline Product volume & $0.05 \mathrm{~cm}^{3}$ & Estimation \\
\hline Density & $2 \mathrm{~g} / \mathrm{cm}^{3}$ & Estimation \\
\hline Absorbed fraction & 1 & Potential dose \\
\hline \multicolumn{3}{|l|}{ Ball pen ${ }^{c)}$} \\
\hline \multicolumn{3}{|l|}{ Contact } \\
\hline Frequency & $365 /$ year & Estimation \\
\hline Total duration & $30 \mathrm{~min}$ & Estimation \\
\hline Use duration & $30 \mathrm{~min}$ & Estimation \\
\hline \multicolumn{3}{|l|}{ Oral } \\
\hline Product volume & $0.2 \mathrm{~cm}^{3}$ & Estimation \\
\hline Density & $1.5 \mathrm{~g} / \mathrm{cm}^{3}$ & Estimation \\
\hline Absorbed fraction & 1 & Potential dose \\
\hline
\end{tabular}

a) For a 4.5 years old child weighting $16.3 \mathrm{~kg}$ - see section 3.2. and Table 3.2.1.

b) For an 18 months old child weighting $9.85 \mathrm{~kg}$ - see section 3.2. and Table 3.2.1.

c) For a 6.5 years old child weighting $20.6 \mathrm{~kg}$ - see section 3.2. and Table 3.2.1.

Source: Modified from the Children's Toys Fact Sheet, RIVM (2002). 
Table 11.13: Default Values on Hand-Mouth Contact

\begin{tabular}{|c|c|c|}
\hline & Default alue & Comments \\
\hline \multicolumn{3}{|l|}{ Piece of chalk ${ }^{\text {a) }}$} \\
\hline \multicolumn{3}{|l|}{ Contact } \\
\hline Frequency & 100/year & Estimate; 2 times per week \\
\hline Total duration & $45 \mathrm{~min}$ & Estimate \\
\hline Use duration & $45 \mathrm{~min}$ & Estimate \\
\hline \multicolumn{3}{|l|}{ Oral } \\
\hline Ingestion rate & $6 \mathrm{mg} / \mathrm{min}$ & $\begin{array}{l}\text { The estimate of the ingestion rate for the piece of chalk is based on } \\
\text { soil, i.e. on the default of } 300 \mathrm{mg} \text { per day (thus an ingestion of } 300 \mathrm{mg} \\
\text { in } 50 \text { minutes). The daily intake is calculated to be } 45 / 50 \times 300=270 \\
\mathrm{mg} \text {. This amount is ingested in } 45 \text { minutes; the ingestion rate is } \\
\text { therefore } 6 \mathrm{mg} / \mathrm{min} \text {. }\end{array}$ \\
\hline Absorbed fraction & 1 & Potential dose \\
\hline \multicolumn{3}{|l|}{ Finger paint ${ }^{b)}$} \\
\hline \multicolumn{3}{|l|}{ Contact } \\
\hline Frequency & 100/year & Estimate; 2 times per week \\
\hline Total duration & $45 \mathrm{~min}$ & Estimate \\
\hline Use duration & $45 \mathrm{~min}$ & Estimate \\
\hline \multicolumn{3}{|l|}{ Oral } \\
\hline Ingestion rate & $30 \mathrm{mg} / \mathrm{min}$ & $\begin{array}{l}\text { Based on the differences between soil and mud, the default value for } \\
\text { products which stick to the skin is first estimated at an amount } 5 \text { times } \\
\text { as large (as soil/chalk), i.e. the ingestion rate for finger paint is estimat- } \\
\text { ed at } 30 \mathrm{mg} / \mathrm{min} \text {. }\end{array}$ \\
\hline Absorbed fraction & 1 & Potential dose \\
\hline \multicolumn{3}{|l|}{ Face paint ${ }^{a)}$} \\
\hline \multicolumn{3}{|l|}{ Contact } \\
\hline Frequency & 12/year & Estimate \\
\hline Total duration & $480 \mathrm{~min}$ & Estimate \\
\hline Use duration & $480 \mathrm{~min}$ & Estimate \\
\hline \multicolumn{3}{|l|}{ Oral } \\
\hline Ingestion rate & $0.44 \mathrm{mg} / \mathrm{min}$ & $\begin{array}{l}\text { For the indirect contact with face paint, it should be noted that it } \\
\text { concerns a substance that sticks to the skin and, in addition to } \\
\text { dermal exposure, is taken in orally via the hands. It is assumed that } \\
\text { the face paint is removed at the end of the day (default: after } 8 \\
\text { hours; } 480 \text { min.). The total amount of face paint on the skin is } 1.4 \mathrm{~g} \\
\text { (see: } \S 5.4 .2 \text { ). We estimate that } 15 \% \text { of this is ingested per day, } \\
\text { which means an ingestion rate of } 210 / 480=0.44 \mathrm{mg} / \mathrm{min} \text {. }\end{array}$ \\
\hline Absorbed fraction & 1 & Potential dose \\
\hline
\end{tabular}

a) For a 4.5 years old child weighting $16.3 \mathrm{~kg}$ - see section 3.2. and Table 3.2.1.

b) For an 18 months old child weighting $9.85 \mathrm{~kg}$ - see section 3.2. and Table 3.2.1.

Source: Modified from the Children's Toys Fact Sheet, RIVM (2002).

With regard to inhalation, the Children's Toys Fact Sheet, RIVM (2002) refers to the more or less volatile organic compounds that evaporate from the toy. The inhalatory exposure to fine particles is described with the "cloud volume" model in section 11.2. This model is developed to estimate exposure to aerosols, but exposure to particles falls within the assumptions of the model. The user of the product that produces particles is assumed, worst case, to have his "nose in the cloud". Default values on inhalation from children's toys are presented in Table 11.14. 
Table 11.14: Default Values on Inhalation from Children's Toys

\begin{tabular}{|c|c|c|}
\hline & Default value & Comments \\
\hline \multicolumn{3}{|l|}{ Dust from chalk ${ }^{a)}$} \\
\hline \multicolumn{3}{|l|}{ Contact } \\
\hline Frequency & 100/year & See Table 12.2.2.3. \\
\hline Total duration & $45 \mathrm{~min}$ & \\
\hline \multicolumn{3}{|l|}{ Inhalation } \\
\hline Emission rate formulation & $11 \mathrm{mg} / \mathrm{min}$ & $\begin{array}{l}\text { It is assumed that } 10 \text { gram of chalk is used. It is estimated } \\
\text { that } 5 \% \text { of the used chalk is released as dust in the air, } \\
\text { resulting in } 500 \mathrm{mg} \text {. If this is released over } 45 \text { minutes, the } \\
\text { average emission rate becomes } 11 \mathrm{mg} / \text { minute. }\end{array}$ \\
\hline Density & $2 \mathrm{~g} / \mathrm{cm}^{3}$ & Estimation \\
\hline Airborne fraction & $0.05 \mathrm{~g} / \mathrm{g}$ & The airborne fraction defines how much of the potential \\
\hline Droplet size & $10 \mu \mathrm{m}$ & $\begin{array}{l}\text { release of particles is actually released into air. No data is } \\
\text { available for the particle size distribution of chalk dust. It } \\
\text { is assumed that the smallest } 10 \% \text { of the dust particles } \\
\text { have an average size of } 25 \mu \mathrm{m} \text {, and that } 5 \% \text { has an aver- } \\
\text { age size of } 10 \mu \mathrm{m} \text {. The default for the airborne fraction is } \\
\text { set to } 0.05 \text {, the default for the particle size to } 10 \mu \mathrm{m} \text { and } \\
\text { the default for the respirable fraction to } 1.7 \% \text {. These } \\
\text { defaults imply that less than } 0.1 \% \text { ( } 0.05 \times 0.017=0.00085 \text { ) } \\
\text { of the chalk dust is available for inhalatory exposure. }\end{array}$ \\
\hline Release height & $100 \mathrm{~cm}$ & Estimation \\
\hline Radius aerosol cloud & $20 \mathrm{~cm}^{3}$ & Estimation \\
\hline Room volume & $20 \mathrm{~m}^{3}$ & \\
\hline Target area & $8 m^{2}$ & Surface room \\
\hline Absorbed fraction & 1 & Potential dose \\
\hline Inhalation rate & $9.2 \mathrm{l} / \mathrm{min}$ & \\
\hline Respirable fraction & $1.7 \%$ & See airborne fraction and droplet size \\
\hline \multicolumn{3}{|l|}{ Dust from blushera) } \\
\hline \multicolumn{3}{|l|}{ Contact } \\
\hline Frequency & 12/year & Estimation \\
\hline Total duration & $480 \mathrm{~min}$ & 8 hours; estimation \\
\hline Use duration & $5 \mathrm{~min}$ & Estimation \\
\hline \multicolumn{3}{|l|}{ Inhalation } \\
\hline Emission rate formulation & $12 \mathrm{mg} / \mathrm{min}$ & $\begin{array}{l}\text { It is estimated that } 300 \mathrm{mg} \text { of blusher is used in } 5 \text { minutes } \\
\text { time. It is assumed that } 20 \% \text { of this amount is released } \\
\text { into air, being } 60 \mathrm{mg} \text {. The emission rate of dust is therefo- } \\
\text { re } 12 \mathrm{mg} / \text { minute. }\end{array}$ \\
\hline Density formulation & $1.8 \mathrm{~g} / \mathrm{cm}^{3}$ & Estimation \\
\hline Airborne fraction & $0.05 \mathrm{~g} / \mathrm{g}$ & See emission rate formulation \\
\hline Droplet size & $10 \mu \mathrm{m}$ & See dust of chalk \\
\hline Release height & $100 \mathrm{~cm}$ & Estimation \\
\hline Radius aerosol cloud & $20 \mathrm{~cm}$ & Estimation \\
\hline Room volume & $20 \mathrm{~m}^{3}$ & \\
\hline Target area & $8 \mathrm{~m}^{2}$ & Surface room \\
\hline Absorbed fraction & 1 & Potential dose \\
\hline Inhalation rate & $9.2 \mathrm{l} / \mathrm{min}$ & \\
\hline Respirable fraction & $1.7 \%$ & See dust of chalk \\
\hline
\end{tabular}

a) For a 4.5 years old child weighting $16.3 \mathrm{~kg}$ - see section 3.2. and Table 3.2.1.

Source: Modified from the Children's Toys Fact Sheet, RIVM (2002).

Children use cosmetics as a game to imitate the behaviour of adults and are therefore exposed to cosmetic products on the skin. The use of cosmetics during play will often take place during dressing up, at children's parties, for example. It will therefore usually occur incidentally and not periodically, as is usual with cosmetics. The sorts of cosmetics usually used are those with which a colour is applied i.e. lipsticks, nail polish, eye shadow and blusher. It is assumed that if cosmetics are used during 
play, these 4 products will be applied one after the other. According to RIVM, it is assumed that the amount that is applied is 3 times as large (unit per surface) as the amount of cosmetics that is usually used (RIVM, 2002). Default values for cosmetics used as children's toy, is presented in Table 11.15. Face paint is described in Table 11.3.

Table 11.15: Default Values for Cosmetics as Children's Toys - Application on Skin

\begin{tabular}{|c|c|c|}
\hline & Default value & Comments \\
\hline \multicolumn{3}{|l|}{ Lipstick $^{\text {a) }}$} \\
\hline \multicolumn{3}{|l|}{ Contact } \\
\hline Frequency & $12 x /$ year & Estimation \\
\hline Use duration & $3 \mathrm{~min}$ & Estimation \\
\hline Total duration & $240 \mathrm{~min}$ & 4 hours; estimation \\
\hline \multicolumn{3}{|l|}{ Oral } \\
\hline Amount product & $0.03 \mathrm{~g}$ & Estimation based on the use of cosmetics by adults - see Table 11.2.1.1. \\
\hline Density & $1.3 \mathrm{~g} / \mathrm{cm}^{3}$ & Estimation \\
\hline Absorbed fraction & 1 & Potential dose \\
\hline \multicolumn{3}{|l|}{ Nail polish $^{\text {a) }}$} \\
\hline \multicolumn{3}{|l|}{ Contact } \\
\hline Frequency & $12 x /$ year & Estimation \\
\hline Use duration & $5 \mathrm{~min}$ & Estimation \\
\hline Total duration & $480 \mathrm{~min}$ & 8 hours; estimation \\
\hline \multicolumn{3}{|l|}{ Dermal } \\
\hline Amount product & $0.75 \mathrm{~g}$ total, $0.25 \mathrm{~g}$ on skin & $1 / 3$ on skin, $2 / 3$ on nail, estimation \\
\hline Density & $0.9 \mathrm{~g} / \mathrm{cm}^{3}$ & Estimation \\
\hline Exposed area & $10 \mathrm{~cm}^{2}$ & On skin, estimation \\
\hline Blood volume & $1 \mathrm{~cm}^{3}$ & \\
\hline Skin blood flow & $0.14 \mathrm{~cm}^{3} / \mathrm{min}$ & \\
\hline \multicolumn{3}{|l|}{ Eye shadow ${ }^{\text {a) }}$} \\
\hline \multicolumn{3}{|l|}{ Contact } \\
\hline Frequency & $12 x /$ year & Estimation \\
\hline Use duration & $5 \mathrm{~min}$ & Estimation \\
\hline Total duration & $480 \mathrm{~min}$ & 8 hours; estimation \\
\hline \multicolumn{3}{|l|}{ Dermal } \\
\hline Amount product & $0.03 \mathrm{~g}$ & $\begin{array}{l}\text { Estimation based on the use of cosmetics by adults - see Table } \\
\text { 11.2.1.1. }\end{array}$ \\
\hline Density & $1.3 \mathrm{~g} / \mathrm{cm}^{3}$ & Estimation \\
\hline Exposed area & $8 \mathrm{~cm}^{2}$ & Estimation \\
\hline Blood volume & $0.8 \mathrm{~cm}^{3}$ & \\
\hline Skin blood flow & $0.11 \mathrm{~cm}^{3} / \mathrm{min}$ & \\
\hline \multicolumn{3}{|l|}{ Blusher $^{a}$ ) } \\
\hline \multicolumn{3}{|l|}{ Contact } \\
\hline Frequency & $12 x /$ year & Estimation \\
\hline Use duration & $5 \mathrm{~min}$ & Estimation \\
\hline Total duration & $480 \mathrm{~min}$ & 8 hours; estimation \\
\hline \multicolumn{3}{|l|}{ Dermal } \\
\hline Amount product & $0.3 \mathrm{~g}$ total & $3 \mathrm{mg} / \mathrm{cm}^{2}$ \\
\hline Density & $1.8 \mathrm{~g} / \mathrm{cm}^{3}$ & Estimation \\
\hline Exposed area & $100 \mathrm{~cm}^{2}$ & Estimation \\
\hline Blood volume & $10 \mathrm{~cm}^{3}$ & \\
\hline Skin blood flow & $1.4 \mathrm{~cm}^{3} / \mathrm{min}$ & \\
\hline
\end{tabular}

a) For a 4.5 years old child weighting $16.3 \mathrm{~kg}$ - see section 3.2. and Table 3.2.1.

Source: Modified from the Children's Toys Fact Sheet, RIVM (2002).

Children are exposed to intensive hand contact via playing with finger paint and modelling clay. In the Children's Toys Fact Sheet, RIVM (2002), it assumed that the product is continuously present on the hands. Since there is intensive hand contact for a prolonged period of time, the total amount of clay is taken as the amount of product. The crucial difference is the age of the users: finger paint will be used by much younger chil- 
dren than for modelling clay. Table 11.16 presents default values on intensive hand contact during play.

Table 11.16: Default Values on Intensive Hand Contact during Play

\begin{tabular}{lrr}
\hline & Default value & Comments \\
\hline Modelling clay ${ }^{\text {a) }}$ & & \\
Contact & & \\
Frequency & $52 \times /$ year & Estimation \\
Use duration & $60 \mathrm{~min}$ & Estimation \\
Total duration & $60 \mathrm{~min}$ & Estimation \\
Dermal & & \\
Amount product & $350 \mathrm{~g}$ & Estimation \\
Density & $2 \mathrm{~g} / \mathrm{cm}^{3}$ & Estimation \\
Exposed area & $390 \mathrm{~cm}^{2}$ & Area hands \\
Blood volume & $39 \mathrm{~cm}^{3}$ & \\
Skin blood flow & $5.5 \mathrm{~cm}^{3} / \mathrm{min}^{2}$ & \\
Finger paint & & \\
Contact & & \\
Frequency & & \\
Use duration & $100 \mathrm{x} / \mathrm{year}$ & Estimation \\
Total duration & $45 \mathrm{~min}$ & Estimation \\
Dermal & $45 \mathrm{~min}$ & Estimation \\
Amount product & & \\
Density & $20 \mathrm{~g}$ & Estimation \\
Exposed area & $1.3 \mathrm{~g} / \mathrm{cm}^{3}$ & Estimation \\
Blood volume & $124 \mathrm{~cm}^{2}$ & $1 / 2$ area hands \\
Skin blood flow & $12 \mathrm{~cm}^{3}$ & \\
& $1.7 \mathrm{~cm}^{3} / \mathrm{min}^{\text {bin }}$ &
\end{tabular}

a) For a 4.5 years old child weighting $16.3 \mathrm{~kg}$ - see section 3.2. and Table 3.2.1.

b) For an 18 months old child weighting $9.85 \mathrm{~kg}$ - see section 3.2. and Table 3.2.1.

Source: Modified from the Children's Toys Fact Sheet, RIVM (2002).

\subsection{US-EPA}

In the Exposure Factors Handbook (US-EPA, 2009), information on the amount of product used, frequency of use, and duration of use for various consumer products typically found in consumer households, is provided. However, due to the large range and variation among consumer products and their exposure pathways, no recommendations on specific exposure values are given.

No data on consumer product from the US will be included in this report. This is because this report is directed primarily towards the exposure factors to be used in exposure assessments in EU in the context of REACH and therefore, the focus will be on the numerous and well validated European data. 


\subsection{WHO}

No recommendations on consumer products are provided by WHO.

\subsection{Conclusion and Recommendations}

RIVM has provided Fact Sheets with default values on several consumer products which are calculated using the ConsExpo software model. The default values in the fact sheets have been collected for consumers (private or non-professional users). They are not aimed at describing exposure for people who professionally work with consumer products, such as hairdressers and in hospitals, for example. These fact sheets, therefore, only describes consumer products which are available to the consumer for private use.

In the fact sheets provided by the RIVM, the basis for the calculation and/or estimation of the default parameter values is a realistic worstcase scenario, and considers consumers who frequently use a certain product under relatively less favourable circumstances. For example, when using a cosmetic product, basic assumptions are: relatively frequent use, application of a relatively large amount in a small room with a low ventilation rate, and a relatively long stay in that room. The parameter values in the fact sheets are aimed at (Dutch) consumers. They are chosen such that a relatively high exposure and uptake are calculated, in the order of magnitude of a 99th percentile of the distribution. To achieve this goal, the $75^{\text {th }}$ or the $25^{\text {th }}$ percentile is calculated (or estimated) for each parameter. The $75^{\text {th }}$ percentile is used for parameters which give a higher exposure for higher values, and the $25^{\text {th }}$ percentile is used in the reverse case (RIVM, 2006a).

Therefore, circumstances are chosen in such a way that it will lead to relatively high exposure. In order to assess the exposed area, RIVM uses the default value for surface areas from the General Fact Sheets, RIVM (2006a) (see section 3.1) and the Exposure Factors Handbook, US-EPA, (1997) (see section 3.2).

No data on consumer product from the US has been included in this report as the primary focus is on the exposure factors to be used in the context of REACH and there are numerous European data.

No recommendations on consumer products are provided by WHO.

In conclusion, the European recommendations are considered as being valid values for consumer products and are therefore, recommended as the default exposure factors for assessments of the European population in the context of REACH. 


\section{Summary, Conclusions and Recommendations}

The aim of an exposure assessment is to determine the nature and extent of contact with chemical substances experienced or anticipated under different conditions. An exposure assessment is the quantitative or qualitative evaluation of the amount of a substance that humans come into contact with and includes consideration of the intensity, frequency and duration of contact, the route of exposure (e.g., dermal, oral or respiratory), rates (chemical intake or uptake rates), the resulting amount that actually crosses the boundary (a dose), and the amount absorbed (internal dose).

In order to perform an exposure assessment, it is necessary to apply various "non-chemical-specific" exposure related parameters such as e.g., body weight, body surface area, activity factors, ventilation rates, ingestion of water/food etc. These parameters are called non-chemicalspecific exposure factors and are generally drawn from the scientific literature or governmental statistics.

The approach to exposure assessment is not as internationally harmonised as hazard assessment. Although broad consistency in the overall approaches used by different bodies and countries in conducting exposure assessment exists, there is variation in the types of approaches and tools used, including the use of exposure factors.

The purpose of this report is to give an overview of non-chemicalspecific exposure factors to be used by the authorities during the process of assessing exposure to both adults and children as well as of risk assessments in the context of the EU chemical regulation "REACH", and to contribute towards a further harmonisation of such exposure factors to be used in exposure assessments. Thus, the process of exposure assessment in itself is not further addressed in this report.

Guidance and recommendations provided by various EU bodies, with the main focus on the information gathered in the REACH "Guidance on Information Requirements and Chemical Safety Assessment" (REACH TGD) published by the European Chemicals Agency (ECHA) (ECHA, 2010), have primarily been addressed as this report is directed primarily towards the exposure factors to be used in exposure assessments in the context of the EU chemical regulation "REACH". The US guidance and recommendations are also addressed as the most recent version of the US-EPA Exposure Factor Handbook, currently (October 2010) available in the form of an external review draft (US-EPA, 2009) provides the most comprehensive overview, considerations, evaluations and recommenda- 
tions in the area of non-chemical-specific exposure factors. In addition, guidance and recommendations provided by the WHO are addressed as this report also is meant to contribute towards a further harmonisation of exposure factors to be used in exposure assessments.

The following non-chemical-specific exposure factors are addressed in this report:

- Body weight

- Body surface areas

- Inhalation rates

- Ingestion of drinking water

- Intake of food

- Ingestion of soil and dust

- Non-dietary ingestion factors

- Lifetime expectancy

- Activity factors

- Consumer products

In general, the data presented in the US-EPA Exposure Factors Handbook (EFH) (US-EPA, 2009) are the most comprehensive, whereas the European data are more limited as are the WHO data. The US guidance and recommendations will be addressed using a recent US-EPA Exposure Factor Handbook, (December 2010) available in the form of an external review draft (US-EPA, 2009), which provides the most comprehensive overview and data basis, considerations, evaluations and recommendations in the area of non-chemical-specific exposure factors. The revised Exposure Factor Handbook was published in September 2011, after the finalisation of the work in this Nordic report.

The US-EPA recommended values are very well validated. The USEPA has assigned a confidence rating of low, medium, or high to each recommended value (US-EPA, 2009). The US-EPA has underscored that this qualitative rating was not intended to represent uncertainty analyses but to represent the US-EPA's judgment on the quality of the underlying data used to derive the recommendations. The judgment was made using five so-called "general assessment factors" (GAFs, described in Section 1.2). The US-EPA noted that there is a continuum from low to high, and that judgment was used to assign a rating to each factor. The recommended values presented in the EFH (US-EPA, 2009) are accompanied by a discussion of the rationale for their rating.

The US-EPA recommendations (US-EPA, 2009) have formed the basis for the recommended values in this report for many of the human exposure factors. One reason is that the US-EPA recommended values generally are based on the most comprehensive and well validated data, whereas the European data are more scarce and limited. Furthermore, the US-EPA recommended values are considered as being the most valid values for most of the human exposure factors as these values in general 
are based on analyses of more recent data than the European data, which in general are based on older references (published more than 10 years ago). In addition, the US-EPA recommended values in general are considered as being representative for Europeans as well for many of the human exposure factors despite that Americans might be different to Europeans in some ways. Finally, harmonisation of default values for exposure factors to be used in exposure assessments would be desirable, i.e., the ideal situation. As this report is also meant to contribute towards a further harmonisation of human exposure factors to be used in exposure assessments, the most comprehensive and well validated data in most cases have been selected as the basis for the recommended values for each exposure factor in this report regardless whether the data are European or American.

In the following sections, the recommended values for the nonchemical-specific exposure factors addressed in this report are presented. Although the focus of this report is to recommend values for nonchemical-specific exposure factors to be used in the context of the EU chemicals regulation "REACH", the recommended values can also be used in the context of other chemical regulations such as those for e.g., biocides, pesticides, cosmetics, toys etc. The percentiles chosen is a decision that has to be made by the assessor depending on the purpose of the assessment. For transparency, the reasoning for the choice of values should always be available in the assessment.

\subsection{Body weight}

The data presented in the US-EPA EFH (US-EPA, 2009) are the most comprehensive. The European data are more limited as are the WHO data.

Americans tend, in average to weigh more than Europeans. Despite this, the US-EPA recommended values for children body weights are considered as being representative for European children as well. Furthermore, the US-EPA recommended values are considered as being the most valid values for body weights as today as these recommendations are based on analyses of the most recent data (NHANES 1999-2006), whereas the European data are predominantly based on older references (ICRP, 1975; US-EPA, 1997; UK Statistics from 1999), or no references have been provided for the recommended values (e.g., REACH TGD Chapter R15). For adults, the currently most used value for average body weight of $70 \mathrm{~kg}$ for men and women combined is probably more representative for Europeans than the average US adult body weight of $80 \mathrm{~kg}$. However, also in Europe, the adult body weight is increasing and therefore, probably approaching the American body weights of today.

In conclusion, the US-EPA recommended values presented in Table 2.4 are considered as being the most valid values for body weight as today and are therefore recommended as default exposure factors for assess- 
ments of European children in the context of REACH. For adults, however, the currently most used value for average body weight of $70 \mathrm{~kg}$ for men and women combined is still recommended as the default value for exposure assessments of the European population in the context of REACH. In addition to the average body weight for men and women combined, a default value of $70 \mathrm{~kg}$ for men and of $60 \mathrm{~kg}$ for women is recommended.

\subsection{Body Surface Area}

The data presented in the US-EPA EFH (US-EPA, 2009) as well as the European data are comprehensive. No recommendations have been provided by the WHO.

Americans tend, in average, to weigh more and thus to have a greater body surface area than Europeans. Despite this, the US-EPA recommended values for body surface areas are considered as being representative for Europeans as well. Furthermore, the US-EPA recommended values are considered as being the most valid values for body surface areas as today as these recommendations are based on analyses of the most recent data (NHANES 1999-2006), whereas the European data are predominantly based on older data, primarily the 1997 version of the EFH (US-EPA, 1997). However, also in Europe, the adult body weight is increasing and thus, also the body surface area and therefore, probably approaching the American body weights and body surface area of today.

In conclusion, the US-EPA recommended values presented in Table 3.2 and 3.3 are considered as being the most valid values for body surface areas as today and are therefore recommended as default exposure factors for assessments of the European population in the context of REACH.

\subsection{Inhalation rates}

The data presented in the US-EPA EFH (US-EPA, 2009) are the most comprehensive. The European data are limited as are the WHO data.

Activity levels might be different among US individuals compared to Europeans. Despite this, the US-EPA recommended values for inhalation rates are considered as being representative for Europeans as well. Furthermore, the US-EPA recommended values are considered as being the most valid values for inhalation rates as today as these recommendations are based on four recent key studies published in the period from 2006-2009. In addition, the US-EPA has noted that these key studies represent an improvement upon those previously used for recommended inhalation rates in previous versions of the EFH. In contrast, the European data are predominantly based on older references (ICRP, 1975; AUH 1995, US-EPA, 1997) or no references have been provided for the recommended values (e.g., REACH TGD Chapter R8, Table R. 8-2). One 
European body, namely EFSA (EFSA; 2010) has based their recommended values on the most recent version of the EFH (US-EPA, 2009). It should also be noted that ECETOC has considered that the American values are probably representative of Europeans as well.

In conclusion, the US-EPA recommended values presented in Table 4.5 and 4.6 are considered as being the most valid values for inhalation rates as today and are therefore recommended as default exposure factors for assessments of the European population in the context of REACH.

\subsection{Drinking water}

The data presented in the US-EPA EFH (US-EPA, 2009) are the most comprehensive. The European data are limited as are the WHO data.

The intake of bottled beverages may be more common today than it was thirty years ago. Therefore, the more recent recommendations from the US-EPA (US-EPA, 2009) may be more representative of the intake of tap water as today compared to the recommended values from ECETOC, which have been based upon a thirty-year old survey as it has been described in the 1997 version of the EFH (US-EPA, 1997). However, it is possible that the US population consumes more bottled beverages than the European population and that the US data therefore underestimate the actual intake of tap water consumed by the European population. Despite this, the US-EPA recommended values are considered as being representative for Europeans as well. Furthermore, the US-EPA recommended values are considered as being the most valid values for intake of drinking water as today as these recommendations are based on two very recent key studies published in 2008. In contrast, the European data are predominantly based on older references (ICRP, 1975; US-EPA, 1997).

In conclusion, the US-EPA recommended values presented in Table 5.2 are considered as being the most valid values for intake of drinking water as today and are therefore recommended as default exposure factors for assessments of the European population in the context of REACH.

\subsection{Food Intake}

The data presented in the US-EPA EFH (US-EPA, 2009) as well as the European data are comprehensive, whereas the WHO data are limited.

The Danish data on dietary habits (DTU-FOOD, 2010) are considered as being the most valid values for food consumption rates as these recommendations are based on analyses of the most recent data and are considered to be representative for Europeans in general.

There might be some differences between US and European infants regarding breast milk intake. However, according to ECETOC (ECETOC, 2001), breast milk consumption values for Swedish infants were similar 
to those of US infants. Thus, the more recent US-EPA recommendations (US-EPA, 2009) are considered as being representative for European infants as well.

In conclusion, the Danish data on dietary habits presented in Table 6.1 are considered as being the most valid values for food consumption rates today and are therefore recommended as default exposure factors for assessments of the European population in the context of REACH. For breast milk intake, the US-EPA recommendations presented in Table 6.9 are considered as being the most valid values as today and are therefore recommended as default exposure factors for assessments of European infants in the context of REACH.

\subsection{Soil and Dust Ingestion}

The data presented in the US-EPA EFH (US-EPA, 2009) are the most comprehensive. There are some European data, but the WHO data are limited.

Data on soil and dust ingestion rates are generally limited and variable, and region specific differences in child play and hygienic patterns will likely affect typical soil and dust ingestion rates. Thus, there might be some differences between the US and European population regarding soil and dust ingestion. Even though the US-EPA recommendations are considered as being the most valid values for soil and dust ingestion rates, the recommended values for house dust ingestion for adults and children proposed from RIVM (RIVM, 2008) are considered as being representative for the European population. For children's dust ingestion the ECHA Guidance on information requirements and chemical assessment. Chapter R. 15: Consumer exposure estimation (ECHA. 2008b) also refers to the Dutch data.

In conclusion, the ECHA recommended values presented in Table 7.1 are considered as being the most valid values for soil and dust ingestion ratesin Europe as today and are therefore recommended as default exposure factors for assessments of the European population in the context of REACH.

\subsection{Non-Dietary Ingestion Factors}

The data presented in the US-EPA EFH (US-EPA, 2009) are the most comprehensive. The European data are limited to single values for specific assessment purposes. No recommendations have been provided by the WHO.

There might be some differences between US and European children regarding hand-to-mouth and hand-to-object frequency and duration. Despite this, the US-EPA recommended values are considered as being representative for European children as well. Furthermore, the US-EPA 
recommendations are considered as being the most valid values for nondietary ingestion factors as today despite the overall rating as low, as these recommendations are based on relatively recent key studies published in the period 2001-2008.

In conclusion, the US-EPA recommended values presented in Table 8.1 are considered as being the most valid values for non-dietary ingestion factors as today and are therefore recommended as default exposure factors for assessments of European children in the context of REACH. No studies have been located on mouthing frequency or duration for adults and therefore, no default exposure factors can be recommended.

\subsection{Lifetime Expectance}

Life expectancy is very different for various countries in the world. The very recent data published by the WHO in 2010 are considered as being the most representative for European lifetime expectancy as today.

In conclusion, the WHO recommended values for the European region presented in Table 9.2 are considered as being the most valid values for lifetime expectancy as today and are therefore recommended as default exposure factors for assessments of the European population in the context of REACH.

\subsection{Activity Factors}

The data presented in the US-EPA EFH (US-EPA, 2009) are the most comprehensive. The European data are limited. No recommendations have been provided by the WHO.

Activity levels might be different among US individuals compared to Europeans. Despite this, the US-EPA recommended values are considered as being representative for Europeans as well. Furthermore, the US-EPA recommendations are considered as being the most valid values for activity factors. It should be noted that the recommended values in the 2009 version of the EFH (US-EPA, 2009) are based on two key studies published in 1991 and 1996 and the ECETOC recommendations are based on different sources published in the 1990'ies.

In conclusion, the US-EPA recommended values presented in Table 10.2 are considered as being the most valid values for activity factors for Europeans as today and are therefore recommended as default exposure factors for assessments of European inhabitants in the context of REACH. No default values for working activities are recommended by the US-EPA as the working environment is not the responsibility of USEPA. For working activities, the recommended values presented in Table 10.1 are recommended as default exposure factors for assessments of European workers in the context of REACH. 


\subsection{Consumer Products}

RIVM has provided Fact Sheets with default values on several consumer products which are calculated using the ConsExpo software model. The default values in the fact sheets have been collected for Dutch consumers (non-professional users). They are not aimed at describing exposure for people who professionally work with consumer products, such as hairdressers and in hospitals, for example. These fact sheets, therefore, only describes consumer products which are available to the consumer for private use. The parameter values in the fact sheets are chosen such that a relatively high exposure and uptake are calculated, in the order of magnitude of a 99th percentile of the distribution. To achieve this goal, the $75^{\text {th }}$ or the $25^{\text {th }}$ percentile is calculated (or estimated) for each parameter. The $75^{\text {th }}$ percentile is used for parameters which give a higher exposure for higher values, and the $25^{\text {th }}$ percentile is used in the reverse case (RIVM, 2006a). In order to assess the exposed area, RIVM uses the default value for surface areas from the General Fact Sheets (RIVM, 2006a) (see Section 3.1) and the Exposure Factors Handbook (US-EPA, 1997) (see Section 3.2).

No data on consumer product from the US has been included in this report as the primary focus is on the exposure factors to be used in the context of REACH and there are numerous European data.

In conclusion, the European recommendations are considered as being valid values for consumer products and are therefore, recommended as the default exposure factors for assessments of the European population in the context of REACH. 


\section{Annex A.}

\section{Body Weight - Supplementary Tables}

\begin{tabular}{|c|c|c|c|}
\hline \multirow[t]{2}{*}{ Region and Country } & Men & Women & Men and Women Combined \\
\hline & \multicolumn{3}{|c|}{ Mean (kg) } \\
\hline \multicolumn{4}{|l|}{ Eastern Europe } \\
\hline Belarus & 75.77 & 69.27 & 72.52 \\
\hline Bulgaria & 61.07 & 53.93 & 57.50 \\
\hline Czech & 75.28 & 65.29 & 70.29 \\
\hline Rep. Hungary & 79.39 & 68.89 & 74.14 \\
\hline Poland & 75.15 & 60.18 & 67.67 \\
\hline Moldova & 75.77 & 69.27 & 72.52 \\
\hline Romania & 61.07 & 53.93 & 57.50 \\
\hline Russia & 75.77 & 69.27 & 72.52 \\
\hline Slovakia & 75.28 & 65.29 & 70.29 \\
\hline Ukraine & 75.77 & 69.27 & 72.52 \\
\hline E. Eur. Average & 74.22 & 66.48 & 70.35 \\
\hline \multicolumn{4}{|l|}{ Northern Europe } \\
\hline Denmark & 83.61 & 68.46 & 76.03 \\
\hline Estonia & 75.77 & 69.27 & 72.52 \\
\hline Finland & 83.61 & 68.46 & 76.03 \\
\hline Iceland & 78.92 & 69.07 & 73.99 \\
\hline Ireland & 77.24 & 67.58 & 72.41 \\
\hline Latvia & 75.77 & 69.27 & 72.52 \\
\hline Lithuania & 75.77 & 69.27 & 72.52 \\
\hline Norway & 78.92 & 69.07 & 73.99 \\
\hline Sweden & 83.61 & 68.46 & 76.03 \\
\hline United Kingdom & 77.24 & 67.58 & 72.41 \\
\hline N. Eur. Average & 78.56 & 67.97 & 73.27 \\
\hline \multicolumn{4}{|l|}{ Southern Europe } \\
\hline Albania & 61.07 & 53.93 & 57.50 \\
\hline Bosnia-Herzegovin & 61.07 & 53.93 & 57.50 \\
\hline Croatia & 61.07 & 53.93 & 57.50 \\
\hline Greece & 76.13 & 66.94 & 71.54 \\
\hline Italy & 73.23 & 62.56 & 67.89 \\
\hline Macedonia FYR & 61.07 & 53.93 & 57.50 \\
\hline Malta & 61.07 & 53.93 & 57.50 \\
\hline Portugal & 61.07 & 53.93 & 57.50 \\
\hline Slovenia & 61.07 & 53.93 & 57.50 \\
\hline Spain & 73.23 & 62.56 & 67.89 \\
\hline Yugoslavia & 75.28 & 60.44 & 67.86 \\
\hline S. Eur. Average & 71.54 & 61.28 & 66.41 \\
\hline \multicolumn{4}{|l|}{ Western Europe } \\
\hline Austria & 79.39 & 68.89 & 74.14 \\
\hline Belgium & 79.78 & 66.38 & 73.08 \\
\hline France & 77.73 & 66.78 & 72.26 \\
\hline Germany & 84.51 & 71.63 & 78.07 \\
\hline Luxemburg & 77.73 & 66.78 & 72.26 \\
\hline Netherlands & 87.80 & 74.37 & 81.08 \\
\hline Switzerland & 79.42 & 67.60 & 73.51 \\
\hline W. Eur. Average & 81.97 & 67.60 & 73.51 \\
\hline Europe Average & 76.46 & 76.46 & 71.51 \\
\hline
\end{tabular}

Source: WHO Global database on Body Mass Index WHO, 1999a; modified from ECETOC, 2001 
Table A.2. Mean and Body Weight from the key study

\begin{tabular}{|c|c|c|c|c|}
\hline \multirow[t]{2}{*}{ Age Group } & \multicolumn{2}{|c|}{ Men } & \multicolumn{2}{|c|}{ Women } \\
\hline & Mean (kg) & $95^{\text {th }}(\mathrm{kg})$ & Mean (kg) & $95^{\text {th }}(\mathrm{kg})$ \\
\hline 0-1 month & 4.9 & 6.8 & 4.6 & 5.9 \\
\hline $1-<3$ months & 6.0 & 7.3 & 5.7 & 7.3 \\
\hline $3-<6$ months & 7.6 & 9.1 & 7.2 & 9.0 \\
\hline $6-<12$ months & 9.4 & 11.5 & 9.0 & 11.2 \\
\hline $1-<2$ years & 11.6 & 14.3 & 11.1 & 13.7 \\
\hline $2-<3$ years & 14.1 & 17.0 & 13.5 & 17.1 \\
\hline $3-<6$ years & 18.8 & 26.2 & 18.3 & 26.2 \\
\hline $6-<11$ years & 31.9 & 51.4 & 31.7 & 53.4 \\
\hline $11-<16$ years & 57.6 & 91.8 & 55.9 & 86.3 \\
\hline $16-<21$ years & 77.3 & 113.0 & 65.9 & 99.7 \\
\hline $21-<30$ years & 84.9 & 123.0 & 71.9 & 109.0 \\
\hline $30-<40$ years & 87.0 & 124.0 & 74.8 & 113.0 \\
\hline $40-<50$ years & 90.5 & 125.0 & 77.1 & 118.0 \\
\hline $50-<60$ years & 89.5 & 123.0 & 77.5 & 117.0 \\
\hline $60-<70$ years & 89.1 & 120.0 & 76.8 & 112.0 \\
\hline $70-<80$ years & 83.9 & 113.0 & 70.8 & 98.2 \\
\hline$\geq 80$ years & 76.1 & 100.0 & 64.1 & 89.1 \\
\hline
\end{tabular}

Source: US-EPA analysis of NHANES, 1999-2006 data, modified from US-EPA, 2009.

Table A.3. A Recent Relevant Body Weight Study Representative for the US Population

\begin{tabular}{lrr}
\hline Age Group & Mean $\mathbf{( k g )}$ & $\mathbf{9 5}^{\text {th }}$ Percentile $^{-}$ \\
\hline $0-1$ month & 4 & 5 \\
$1-<3$ months & 5 & 7 \\
$3-<6$ months & 7 & 10 \\
$6-<12$ months & 9 & 12 \\
$1-<2$ years & 12 & 15 \\
$2-<3$ years & 14 & 19 \\
$3-<6$ years & 18 & 25 \\
$6-<11$ years & 30 & 45 \\
$11-<16$ years & 54 & 82 \\
$16-<18$ years & 67 & 100 \\
$18-<21$ years & 69 & 100 \\
$21+$ & 76 & 107 \\
$65+$ & 72 & 100 \\
\hline
\end{tabular}

Source: Kahn and Stralka, 2008, modified from US-EPA, 2009.

Table A.4. Neonatal Weight by Gestational Age for Men and Women Combined

Gestational Age (weeks)

Weight (g)

$50^{\text {th }}$ Percentile

$95^{\text {th }}$ Percentile

\begin{tabular}{lrr}
\hline 25 & 660 & 968 \\
26 & 760 & 1,103 \\
27 & 875 & 1,257 \\
28 & 1,005 & 1,430 \\
29 & 1,153 & 1,623 \\
30 & 1,319 & 1,836 \\
31 & 1,502 & 2,070 \\
32 & 1,702 & 2,321 \\
33 & 1,918 & 2,587 \\
34 & 2,146 & 2,865 \\
35 & 2,383 & 3,148 \\
36 & 2,622 & 3,428 \\
37 & 2,859 & 3,698 \\
38 & 3,083 & 3,947 \\
39 & 3,288 & 4,164 \\
40 & 3,462 & 4,340 \\
41 & 3,597 & 4,462 \\
42 & 3,685 & 4,523 \\
43 & 3,717 & 4,515 \\
\hline
\end{tabular}

Source: Doubilet et al., 1997, modified from US-EPA, 2009. 


\section{Annex B.}

\section{Body Surface Area - Supplementary Tables}

\begin{tabular}{|c|c|c|c|c|c|c|}
\hline \multirow{3}{*}{$\begin{array}{l}\text { Weight (kg) } \\
\text { Surface Area }\end{array}$} & \multicolumn{2}{|c|}{ Men } & \multicolumn{2}{|c|}{ Women } & \multicolumn{2}{|c|}{ Men and Women Combined } \\
\hline & \multicolumn{2}{|c|}{74} & \multicolumn{2}{|c|}{61} & \multicolumn{2}{|l|}{64} \\
\hline & $m^{2}$ & Percent & $\mathrm{m}^{2}$ & Percent & $\mathrm{m}^{2}$ & Percent \\
\hline Head & 0.116 & 6.6 & 0.122 & 6.4 & 0.113 & 6.7 \\
\hline Trunk & 0.630 & 36 & 0.695 & 36.4 & 0.585 & 34.8 \\
\hline Arms & 0.245 & 14 & 0.273 & 14.3 & 0.232 & 13.8 \\
\hline Hands & 0.086 & 4.9 & 0.094 & 4.9 & 0.082 & 4.9 \\
\hline Legs & 0.560 & 32 & 0.602 & 31.5 & 0.553 & 32.9 \\
\hline Feet & 0.117 & 6.7 & 0.124 & 6.5 & 0.116 & 6.9 \\
\hline Total & 1.75 & 100 & 1.91 & 100 & 1.68 & 100 \\
\hline
\end{tabular}

Source: Modified from RIVM, 2006a

Table B.2. Default values of body weight and body surface of children - as Presented by RIVM

\begin{tabular}{lrrrrrr}
\hline \multirow{2}{*}{ Age } & $\begin{array}{r}\text { Body } \begin{array}{r}\text { Weight } \\
(\mathbf{k g})\end{array} \\
\end{array}$ & & $\begin{array}{r}\text { Body Surface } \\
\left(\mathbf{m}^{2}\right)\end{array}$ & & \multicolumn{3}{l}{ Body Surface in Percent } \\
\cline { 5 - 7 } & & & Head & Trunk & Arms+Hands & Legs+Feet \\
\hline 1.5 months & 4.30 & 0.270 & 20.4 & 32.2 & 16.9 & 30.5 \\
4.5 months & 6.21 & 0.346 & 19.5 & 32.8 & 17.2 & 30.5 \\
7.5 months & 7.62 & 0.398 & 18.5 & 33.5 & 17.4 & 30.6 \\
10.5 months & 8.69 & 0.437 & 17.6 & 34.1 & 17.7 & 30.6 \\
13.5 months & 9.47 & 0.467 & 16.9 & 34.3 & 17.9 & 30.9 \\
1.5 year & 9.85 & 0.480 & 16.2 & 34.0 & 18.15 & 31.65 \\
2.5 years & 12.5 & 0.575 & 14.8 & 33.6 & 18.65 & 32.95 \\
3.5 years & 14.1 & 0.640 & 14.05 & 33.35 & 19.1 & 33.5 \\
4.5 years & 16.3 & 0.709 & 13.4 & 33.05 & 19.5 & 34.05 \\
6.5 years & 20.6 & 0.841 & 12.5 & 33.45 & 19.45 & 34.55 \\
9.5 years & 28.4 & 1.05 & 11.2 & 33.55 & 19.3 & 35.95 \\
12.5 years & 39.3 & 1.31 & 9.8 & 33.15 & 19.6 & 37.4 \\
13.5 years & 43.9 & 1.40 & 9.4 & 32.75 & 20.0 & 37.8 \\
16.5 years & 56.8 & 1.65 & 8.3 & 31.65 & 21.35 & 38.65 \\
17.5 years & 58.2 & 1.67 & 8.05 & 32.1 & 21.0 & 38.8 \\
\hline
\end{tabular}

Source: Modified from RIVM, 2006a.

Table B.3. Default values of body weight and body surface of children - as Presented by RIVM

\begin{tabular}{lrrrrrrrr}
\hline \multirow{2}{*}{ Age } & \multirow{2}{*}{$\begin{array}{r}\text { Body Weight } \\
(\mathbf{k g})\end{array}$} & $\begin{array}{r}\text { Body Surface } \\
\left(\mathbf{m}^{2}\right)\end{array}$ & \multicolumn{5}{c}{ Body surface in Percent } \\
\cline { 5 - 9 } & & & Head & Trunk & Arms & Hands & Legs & Feet \\
\hline 4.5 months & 6.21 & 0.346 & 19.5 & 32.8 & 12.1 & 5.1 & 23.5 & 7.0 \\
7.5 months & 7.62 & 0.398 & 18.5 & 33.5 & 12.2 & 5.2 & 23.6 & 7.0 \\
13.5 months & 9.47 & 0.467 & 16.9 & 34.3 & 12.6 & 5.3 & 23.8 & 7.1 \\
1.5 year & 9.85 & 0.480 & 16.2 & 34.0 & 13.0 & 5.15 & 25.05 & 6.6 \\
4.5 years & 16.3 & 0.709 & 13.4 & 33.05 & 14.0 & 5.5 & 26.95 & 7.1 \\
6.5 years & 20.6 & 0.841 & 12.5 & 33.45 & 13.95 & 5.5 & 27.35 & 7.2 \\
12.5 years & 39.3 & 1.310 & 9.8 & 33.15 & 13.9 & 5.7 & 30.0 & 7.4 \\
\hline
\end{tabular}

Source: Modified from RIVM, 2006a. 
Table B.4. Skin Surface Area Distributions based upon Lognormal Body Weight Distributions

\begin{tabular}{lcr}
\hline Age group and Surface Area Equation & Mean $\left(\mathbf{m}^{2}\right)$ & $\mathbf{9 5}^{\text {th }}$ Percentile $\left(\mathbf{m}^{2}\right)$ \\
\hline Men & & 2.19 \\
Costeff & 1.91 & 2.51 \\
Burmaster & 2.08 & 0.70 \\
Women, 2 years & & 0.70 \\
Costeff & 0.59 & \\
Burmaster & 0.60 & 0.72 \\
Men, 2 years & & 0.74 \\
Costeff & 0.62 & 1.51 \\
Burmaster & 0.64 & 1.49 \\
Women, 10 years & & 1.45 \\
Costeff & 1.20 & 1.48 \\
Burmaster & 1.17 & \\
Men, 10 years & & 1.19 \\
Costeff & 1.12 & \\
Burmaster & & \\
\hline
\end{tabular}

Source: Health Survey for England (HSE) Data (1996 for Adult Males, 1995-1997 for Children), modified from ECETOC, 2001.

Table B.5. Percentage of Total Surface Area that is Exposed for Various Exposure Scenarios

\begin{tabular}{ll}
\hline Scenario & $\begin{array}{l}\text { US-EPA Recommendations } \\
\text { for Percentage Exposed (\%) }\end{array}$ \\
\hline Swimming and bathing & $75-100$ \\
Adult outdoor exposure (only head and hands exposed) & 10 \\
Adult outdoor exposure (head, hands, forearms and lower legs exposed) & 25 \\
Moderate climate, winter & 5 \\
Moderate climate, spring and fall & 10 \\
Moderate climate, summer & 25 \\
\hline
\end{tabular}

Source: US-EPA, 1997, modified from ECETOC, 2001

Table B.6. Default Values of Protection Factor for different Outfits

\begin{tabular}{ll}
\hline Description of clothes & $\begin{array}{l}\text { Default Protection } \\
\text { Factor }\end{array}$ \\
\hline $\begin{array}{ll}\text { Clothing penetration (only for dry substances }{ }^{\text {a) }} \text { ) - for a non-professional wearing: } \\
\text { long-sleeved shirt and trousers or skirt with shoes; no gloves worn (central tendency) }\end{array}$ & $50 \%$ protection \\
Wearing protective gloves & $90 \%$ protection \\
Wearing drya) cotton coveralls & $75 \%$ protection \\
Wearing "impermeable" coveralls & $95 \%$ protection \\
\hline
\end{tabular}

a) Only for dry substances. Dry is introduced here, since wet cotton coveralls will offer little or no protection. 
Table B.7. Percentage of Total Body Surface by Body Part for Children (Genders Combined) and Adults by Gender

\begin{tabular}{|c|c|c|c|c|c|c|}
\hline \multirow[t]{2}{*}{ Age (years) } & Head & Trunk & Arms & Hands & Legs & Feet \\
\hline & \multicolumn{6}{|c|}{ Mean (\%) } \\
\hline \multicolumn{7}{|l|}{ Children } \\
\hline$<1$ & 18.2 & 35.7 & 13.7 & 5.3 & 20.6 & 6.5 \\
\hline $1<2$ & 16.5 & 35.5 & 13.0 & 5.7 & 23.1 & 6.3 \\
\hline $2<3$ & 14.2 & 38.5 & 11.8 & 5.3 & 23.2 & 7.1 \\
\hline $3<4$ & 13.6 & 31.9 & 14.4 & 6.1 & 26.8 & 7.2 \\
\hline $4<5$ & 13.8 & 31.5 & 14.0 & 5.7 & 27.8 & 7.3 \\
\hline $5<6$ & - & - & - & - & - & . \\
\hline $6<7$ & 13.1 & 35.1 & 13.1 & 4.7 & 27.1 & 6.9 \\
\hline $7<8$ & - & - & - & - & - & 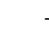 \\
\hline $8<9$ & - & - & - & - & - & 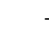 \\
\hline $9<10$ & 12.0 & 34.2 & 12.3 & 5.3 & 28.7 & 7.6 \\
\hline $10<11$ & - & - & - & - & - & 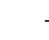 \\
\hline $11<12$ & - & - & - & - & - & . \\
\hline $12<13$ & 8.7 & 34.7 & 13.7 & 5.4 & 30.5 & 7.0 \\
\hline $13<14$ & 10.0 & 32.7 & 12.1 & 5.1 & 32.0 & 8.0 \\
\hline $14<15$ & - & - & - & - & - & . \\
\hline $15<16$ & - & - & - & - & - & . \\
\hline $16<17$ & 8.0 & 32.7 & 13.1 & 5.7 & 33.6 & 6.9 \\
\hline $17<18$ & 7.6 & 31.7 & 17.5 & 5.1 & 30.8 & 7.3 \\
\hline \multicolumn{7}{|l|}{ Adults } \\
\hline Men, 18+ & 7.8 & 35.9 & 14.1 & 5.2 & 31.2 & 7.0 \\
\hline Women, 18+ & 7.1 & 34.8 & 14.0 & 5.1 & 32.4 & 6.5 \\
\hline
\end{tabular}

Table B.8. Surface Area of Adults (21+ Years) in Square Meters

\begin{tabular}{|c|c|c|c|c|}
\hline \multirow[t]{2}{*}{ Body Part } & \multicolumn{2}{|c|}{ Men } & \multicolumn{2}{|c|}{ Women } \\
\hline & Mean $\left(\mathrm{m}^{2}\right)$ & $95^{\text {th }}$ Percentile $\left(\mathrm{m}^{2}\right)$ & Mean $\left(\mathrm{m}^{2}\right)$ & $95^{\text {th }}$ Percentile $\left(\mathrm{m}^{2}\right)$ \\
\hline Head & 0.136 & 0.149 & 0.114 & 0.121 \\
\hline Trunk (incl. Neck) & 0.827 & 1.020 & 0.654 & 0.850 \\
\hline Upper Extremities & 0.393 & 0.456 & 0.304 & 0.354 \\
\hline Arms & 0.314 & 0.379 & 0.237 & 0.266 \\
\hline Upper Arms & 0.172 & 0.205 & - & - \\
\hline Forearms & 0.148 & 0.181 & - & - \\
\hline Hands & 0.107 & 0.124 & 0.089 & 0.106 \\
\hline Lower Extremities & 0.802 & 0.936 & 0.707 & 0.875 \\
\hline Legs & 0.682 & 0.811 & 0.598 & 0.764 \\
\hline Thighs & 0.412 & 0.495 & 0.364 & 0.479 \\
\hline Lower Legs & 0.268 & 0.312 & 0.233 & 0.286 \\
\hline Feet & 0.137 & 0.156 & 0.122 & 0.146 \\
\hline Total & 2.060 & 2.410 & 1.850 & 2.33 \\
\hline
\end{tabular}

Source: Based on US-EPA, 1985 and NHANES 2005-2006, modified from US-EPA, 2009. 


\section{Annex C.}

\section{Inhalation of air - Supplementary Tables}

Table C.1. Mean and $95^{\text {th }}$ Percentile Inhalation Rate Values $\left(\mathrm{m}^{3} /\right.$ day) for Free-living Normal-weight Men and Women

\begin{tabular}{|c|c|c|c|c|}
\hline \multicolumn{5}{|c|}{ Daily Inhalation Rate - Free-Living Normal-Weight } \\
\hline & \multicolumn{2}{|c|}{ Mean $\mathrm{m}^{3} /$ day } & \multicolumn{2}{|c|}{$95^{\text {th }}$ Percentile $\mathrm{m}^{3} /$ day } \\
\hline & Men & Women & Men & Women \\
\hline \multicolumn{5}{|l|}{ Infants: } \\
\hline $1-<3$ months & 3.38 & 3.26 & 4.57 & 4.36 \\
\hline $3-<6$ months & 3.38 & 3.26 & 4.57 & 4.36 \\
\hline $6-<12$ months & 4.22 & 3.96 & 5.51 & 5.14 \\
\hline Birth to $<1$ year & 3.85 & 3.64 & 5.09 & 4.78 \\
\hline \multicolumn{5}{|l|}{ Children: } \\
\hline $1-<2$ years & 5.12 & 4.78 & 6.56 & 6.36 \\
\hline $2-<3$ years & 7.60 & 7.06 & 9.71 & 8.97 \\
\hline $3-<6$ years & 7.60 & 7.06 & 9.71 & 8.97 \\
\hline $6-<11$ years & 10.59 & 9.84 & 13.87 & 12.61 \\
\hline $11-<16$ years & 17.23 & 13.28 & 23.26 & 17.56 \\
\hline $16-<21$ years & 17.23 & 13.28 & 23.26 & 17.56 \\
\hline \multicolumn{5}{|c|}{ Adults (over 21 years): } \\
\hline $21-<31$ years & 17.36 & 13.45 & 22.65 & 17.50 \\
\hline $31-<41$ years & 16.88 & 13.68 & 21.00 & 16.58 \\
\hline $41-<51$ years & 16.24 & 12.31 & 20.64 & 15.71 \\
\hline $51-<61$ years & 16.24 & 12.31 & 20.64 & 15.71 \\
\hline $61-<71$ years & 14.26 & 11.21 & 18.47 & 14.69 \\
\hline $71-<81$ years & 12.96 & 9.80 & 17.03 & 13.37 \\
\hline$\geq 81$ years & 12.96 & 9.80 & 17.03 & 13.37 \\
\hline
\end{tabular}

Source: Brochu et al., 2006a, modified from US-EPA, 2009.

Table C.2. Physiological Daily Inhalation Rates ( $\mathrm{m}^{3} /$ day) for Free-Living Normal-Weight- Unadjusted by Body Weight

\begin{tabular}{|c|c|c|c|c|c|c|}
\hline \multirow[t]{3}{*}{ Age group (years) } & \multicolumn{2}{|c|}{ Body Weight ${ }^{\mathrm{a}}(\mathrm{kg})$ Mean } & \multicolumn{4}{|c|}{ Physiological Daily Inhalation Rates ${ }^{b}\left(m^{3} /\right.$ day) } \\
\hline & \multirow[b]{2}{*}{ Men } & \multirow[b]{2}{*}{ Women } & \multicolumn{2}{|c|}{ Mean $\left(\mathrm{m}^{3} /\right.$ day) } & \multicolumn{2}{|c|}{$95^{\text {th }}$ Percentile $\left(\mathrm{m}^{3} /\right.$ day $)$} \\
\hline & & & Men & Women & Men & Women \\
\hline $0.22-<0.5$ & 6.7 & 6.5 & 3.38 & 3.26 & 4.57 & 4.36 \\
\hline $0.5-<1$ & 8.8 & 8.5 & 4.22 & 3.96 & 5.51 & 5.14 \\
\hline $1-<2$ & 10.6 & 10.6 & 5.12 & 4.78 & 6.56 & 6.36 \\
\hline $2-<5$ & 15.3 & 14.4 & 7.60 & 7.06 & 9.71 & 8.97 \\
\hline $5-<7$ & 19.8 & 19.7 & 8.64 & 8.22 & 10.66 & 10.38 \\
\hline $7-<11$ & 28.9 & 28.3 & 10.59 & 9.84 & 13.87 & 12.61 \\
\hline $11-<23$ & 58.6 & 50.0 & 17.23 & 13.28 & 23.26 & 17.56 \\
\hline $23-<30$ & 70.9 & 59.2 & 17.48 & 13.67 & 22.11 & 17.42 \\
\hline $30-<40$ & 71.5 & 58.7 & 16.88 & 13.68 & 21.00 & 16.58 \\
\hline $40-<65$ & 71.1 & 58.8 & 16.24 & 12.31 & 20.64 & 15.71 \\
\hline $65-\leq 96$ & 68.9 & 57.2 & 12.96 & 9.80 & 17.03 & 13.37 \\
\hline
\end{tabular}

a) Measured body weight. Normal-weight individuals defined according to the BMI cut-offs.

b) Physiological daily inhalation rates were calculated using the following equation: (TDEE + ECG) ${ }^{*} H^{*}(V E / V O 2) * 10-3$, where $\mathrm{H}=0.21 \mathrm{~L}$ of O2/Kcal, VE/VO2 = 27 (Layton, 1993), TDEE = total daily energy expenditure ( $\mathrm{kcal} / \mathrm{day})$ and $E C G$ = stored daily energy cost for growth (kcal/day). Source: Brochu et al., 2006a, modified from US-EPA, 2009. 
Table C.3. Physiological Daily Inhalation Rates $\left(\mathrm{m}^{3} /\right.$ day) for Free-living Normal-weight and Overweight/obese - Unadjusted for Body Weight

\begin{tabular}{|c|c|c|c|c|c|c|}
\hline \multirow{3}{*}{$\begin{array}{l}\text { Age group } \\
\text { (years) }\end{array}$} & \multicolumn{2}{|c|}{ Body Weight $^{\mathrm{a}}(\mathrm{kg})$ Mean } & \multicolumn{4}{|c|}{ Physiological Daily Inhalation ${ }^{b}\left(m^{3} /\right.$ day) } \\
\hline & \multirow[b]{2}{*}{$\begin{array}{r}\text { Normal- } \\
\text { weight }\end{array}$} & \multirow[b]{2}{*}{$\begin{array}{r}\text { Overweight/ } \\
\text { Obese }\end{array}$} & \multicolumn{2}{|c|}{ Mean $\left(\mathrm{m}^{3} /\right.$ day $)$} & \multicolumn{2}{|c|}{$95^{\text {th }}$ Percentile $\left(\mathrm{m}^{3} /\right.$ day $)$} \\
\hline & & & $\begin{array}{r}\text { Normal- } \\
\text { weight }\end{array}$ & $\begin{array}{r}\text { Oveweight/ } \\
\text { Obese }\end{array}$ & $\begin{array}{r}\text { Normal- } \\
\text { weight }\end{array}$ & $\begin{array}{r}\text { Overweight/ } \\
\text { Obese }\end{array}$ \\
\hline \multicolumn{7}{|l|}{ Men } \\
\hline $4-<5.1$ & 19.0 & 26.5 & 7.90 & 9.59 & 9.50 & 11.66 \\
\hline $5.1-<9.1$ & 22.6 & 32.5 & 9.14 & 10.88 & 11.51 & 14.98 \\
\hline $9.1-<18.1$ & 41.4 & 55.8 & 13.69 & 14.52 & 20.19 & 17.78 \\
\hline $18.1-<40.1$ & 71.3 & 98.1 & 17.41 & 20.39 & 21.85 & 26.35 \\
\hline $40.1-<70.1$ & 70.0 & 93.2 & 15.60 & 17.96 & 20.34 & 24.06 \\
\hline $70.1-\leq 96$ & 68.9 & 82.3 & 12.69 & 14.23 & 16.53 & 19.06 \\
\hline \multicolumn{7}{|l|}{ Women } \\
\hline $4-<5.1$ & 18.7 & 26.1 & 7.41 & 8.70 & 8.90 & 10.56 \\
\hline $5.1-<9.1$ & 25.5 & 34.6 & 9.39 & 10.55 & 12.05 & 14.22 \\
\hline $9.1-<18.1$ & 42.7 & 59.2 & 12.04 & 14.27 & 16.74 & 18.71 \\
\hline $18.1-<40.1$ & 59.1 & 84.4 & 13.73 & 15.66 & 17.04 & 19.13 \\
\hline $40.1-<70.1$ & 59.1 & 81.7 & 11.93 & 13.01 & 15.48 & 17.64 \\
\hline $70.1-\leq 96$ & 54.8 & 69.0 & 8.87 & 10.00 & 11.81 & 12.93 \\
\hline
\end{tabular}

a) Measured body weight. Normal-weight and overweight/obese males defined according to the BMI cut-offs.

b) Physiological daily inhalation rates were calculated using the following equation: (TDEE + ECG)*H*(VE/VO2)*10-3, where $\mathrm{H}=0.21 \mathrm{~L}$ of O2/Kcal, VE/VO2 = 27 (Layton, 1993), TDEE = total daily energy expenditure (kcal/day) and ECG = stored daily energy cost for growth (kcal/day).

Source: Brochu et al., 2006a, modified from US-EPA, 2009.

Table C.4. Physiological Daily Inhalation Rates ( $\mathrm{m}^{3} /$ day) for Free-Living Normal-Weight- Adjusted by Body Weight

\begin{tabular}{|c|c|c|c|c|}
\hline \multirow[t]{3}{*}{ Age group (years) } & \multicolumn{4}{|c|}{ Physiological Daily Inhalation Rates ${ }^{\mathrm{b}}$ ( $\mathrm{m}^{3} / \mathrm{kg}$-day) } \\
\hline & \multicolumn{2}{|c|}{ Mean $\left(\mathrm{m}^{3} / \mathrm{kg}\right.$-day) } & \multicolumn{2}{|c|}{$95^{\text {th }}$ Percentile $\left(\mathrm{m}^{3} / \mathrm{kg}\right.$-day $)$} \\
\hline & Men & Women & Men & Women \\
\hline $0.22-<0.5$ & 0.51 & 0.50 & 0.66 & 0.66 \\
\hline $0.5-<1$ & 0.48 & 0.46 & 0.60 & 0.57 \\
\hline $1-<2$ & 0.48 & 0.45 & 0.58 & 0.58 \\
\hline $2-<5$ & 0.44 & 0.44 & 0.51 & 0.56 \\
\hline $5-<7$ & 0.42 & 0.40 & 0.49 & 0.47 \\
\hline $7-<11$ & 0.37 & 0.35 & 0.47 & 0.45 \\
\hline $11-<23$ & 0.30 & 0.27 & 0.38 & 0.35 \\
\hline $23-<30$ & 0.25 & 0.23 & 0.31 & 0.30 \\
\hline $30-<40$ & 0.24 & 0.24 & 0.29 & 0.29 \\
\hline $40-<65$ & 0.23 & 0.21 & 0.30 & 0.27 \\
\hline $65-\leq 96$ & 0.19 & 0.17 & 0.24 & 0.23 \\
\hline
\end{tabular}

a) Measured body weight. Normal-weight individuals defined according to the BMI cut-offs.

b) Physiological daily inhalation rates were calculated using the following equation: (TDEE + $\mathrm{ECG})^{*} \mathrm{H}^{*}\left(\mathrm{VE} / \mathrm{VO}_{2}\right) * 10-3$, where $\mathrm{H}=0.21 \mathrm{~L}$ of $\mathrm{O}_{2} / \mathrm{Kcal}, \mathrm{VE} / \mathrm{VO}_{2}=27$ (Layton, 1993), TDEE = total daily energy expenditure (kcal/day) and ECG = stored daily energy cost for growth (kcal/day).

Source: Brochu et al., 2006a, modified from US-EPA, 2009. 
Table C.5. Physiological Daily Inhalation Rates ( $\mathrm{m}^{3} /$ day) for Free-living Normalweight and Overweight/obese - Adjusted for Body Weight

\begin{tabular}{|c|c|c|c|c|}
\hline \multirow[t]{3}{*}{ Age group (years) } & \multicolumn{4}{|c|}{ Physiological Daily Inhalation Rates ${ }^{b}$ ( $^{3} / \mathrm{kg}$-day) } \\
\hline & \multicolumn{2}{|c|}{ Mean (m³/kg-day) } & \multicolumn{2}{|c|}{$95^{\text {th }}$ Percentile ( $\mathrm{m}^{3} / \mathrm{kg}$-day) } \\
\hline & Normalweight & Overweight/Obese & Normalweight & Overweight/Obese \\
\hline \multicolumn{5}{|l|}{ Men } \\
\hline $4-<5.1$ & 0.42 & 0.37 & 0.49 & 0.44 \\
\hline $5.1-<9.1$ & 0.41 & 0.35 & 0.50 & 0.47 \\
\hline $9.1-<18.1$ & 0.33 & 0.27 & 0.41 & 0.33 \\
\hline $18.1-<40.1$ & 0.25 & 0.21 & 0.31 & 0.27 \\
\hline $40.1-<70.1$ & 0.22 & 0.19 & 0.29 & 0.25 \\
\hline $70.1-\leq 96$ & 0.19 & 0.17 & 0.24 & 0.22 \\
\hline \multicolumn{5}{|l|}{ Women } \\
\hline $4-<5.1$ & 0.40 & 0.34 & 0.48 & 0.41 \\
\hline $5.1-<9.1$ & 0.37 & 0.32 & 0.47 & 0.43 \\
\hline $9.1-<18.1$ & 0.29 & 0.25 & 0.38 & 0.33 \\
\hline $18.1-<40.1$ & 0.23 & 0.19 & 0.30 & 0.23 \\
\hline $40.1-<70.1$ & 0.20 & 0.16 & 0.27 & 0.21 \\
\hline $70.1-\leq 96$ & 0.16 & 0.15 & 0.22 & 0.19 \\
\hline
\end{tabular}

a) Measured body weight. Normal-weight individuals defined according to the BMI cut-offs.

b) Physiological daily inhalation rates were calculated using the following equation: (TDEE + $\mathrm{ECG})^{*} \mathrm{H}^{*}\left(\mathrm{VE} / \mathrm{VO}_{2}\right) * 10-3$, where $\mathrm{H}=0.21 \mathrm{~L}$ of $\mathrm{O}_{2} / \mathrm{Kcal}, \mathrm{VE} / \mathrm{VO}_{2}=27$ (Layton, 1993), TDEE = total daily energy expenditure $(\mathrm{kcal} / \mathrm{day})$ and $\mathrm{ECG}=$ stored daily energy cost for growth $(\mathrm{kcal} /$ day).

Source: Brochu et al., 2006a, modified from US-EPA, 2009.

Table C.6. Physiological Daily Inhalation Rates for Newborns

\begin{tabular}{|c|c|c|c|}
\hline \multirow[t]{2}{*}{ Age Group } & \multirow{2}{*}{$\begin{array}{l}\text { Body Weight (kg) } \\
\text { Mean } \pm \text { SD }\end{array}$} & \multicolumn{2}{|c|}{ Physiological Daily Inhalation Rates } \\
\hline & & Mean \pm SD $\left(\mathrm{m}^{3} /\right.$ day $)$ & Mean $\pm S D\left(m^{3} / k g-\right.$ day $)$ \\
\hline 21 days $(2 \text { weeks })^{a, c}$ & $1.2 \pm 0.2$ & $0.85 \pm 0.17^{f}$ & $0.74 \pm 0.09^{f}$ \\
\hline 32 days ( 1 month) ${ }^{b, d}$ & $4.7 \pm 0.7$ & $2.45 \pm 0.59^{g}$ & $0.53 \pm 0.10^{g}$ \\
\hline 33 days ( 1 month) ${ }^{a, d}$ & $4.8 \pm 0.3$ & $2.99 \pm 0.47^{g}$ & $0.62 \pm 0.09^{g}$ \\
\hline
\end{tabular}

a) Formula-fed infants.

b) Breast-fed infants.

c) Healthy infants with very low birth weight.

d) Infants evaluated as being clinically healthy and neither underweight or overweight.

e) Physiological daily inhalation rates were calculated using the following equation: (TDEE + $\mathrm{ECG}) * \mathrm{H}^{*}(\mathrm{VE} / \mathrm{VO} 2) * 10-3$, where $\mathrm{H}=0.21 \mathrm{~L}$ of O2/Kcal, VE/VO2 = 27 (Layton, 1993), TDEE = total daily energy expenditure ( $\mathrm{kcal} / \mathrm{day}$ ) and ECG = stored daily energy cost for growth (kcal/day).

f) TDEEs based on nutritional balance measurements during 3-day periods.

g) TDEEs based on $2 \mathrm{H} 2 \mathrm{O}$ and $\mathrm{H} 2180$ disappearance rates from urine.

Source: Brochu et al., 2006a, modified from US-EPA, 2009. 
Table C.7. Daily Average Inhalation Rate for Men and Women, by Age Category ${ }^{\text {a }}$ - Unadjusted by Body Weight

\begin{tabular}{|c|c|c|c|c|}
\hline \multicolumn{5}{|c|}{ Daily Inhalation Rate - Unadjusted for Body Weight } \\
\hline & \multicolumn{2}{|c|}{ Mean $\mathrm{m}^{3} /$ day } & \multicolumn{2}{|c|}{$95^{\text {th }}$ Percentile $\mathrm{m}^{3} /$ day } \\
\hline & Men & Women & Men & Women \\
\hline \multicolumn{5}{|l|}{ Children: } \\
\hline Birth to $<1$ year & 8.76 & 8.52 & 12.69 & 12.66 \\
\hline $1-<2$ years & 13.49 & 13.13 & 17.90 & 18.62 \\
\hline $2-<3$ years & 13.23 & 12.74 & 17.71 & 16.36 \\
\hline $3-<6$ years & 12.64 & 12.17 & 15.41 & 14.93 \\
\hline $6-<11$ years & 13.42 & 12.41 & 17.73 & 16.34 \\
\hline $11-<16$ years & 15.32 & 13.44 & 21.21 & 17.41 \\
\hline $16-<21$ years & 17.21 & 13.59 & 23.37 & 18.29 \\
\hline \multicolumn{5}{|c|}{ Adults (over 21 years): } \\
\hline $21-<31$ years & 18.82 & 14.57 & 27.13 & 21.14 \\
\hline $31-<41$ years & 20.29 & 14.98 & 28.90 & 20.45 \\
\hline $41-<51$ years & 20.94 & 16.20 & 28.37 & 21.34 \\
\hline $51-<61$ years & 20.91 & 16.19 & 29.09 & 21.21 \\
\hline $61-<71$ years & 17.94 & 12.99 & 23.50 & 16.14 \\
\hline $71-<81$ years & 16.34 & 12.04 & 20.42 & 15.19 \\
\hline$\geq 81$ years & 15.15 & 11.15 & 18.69 & 13.94 \\
\hline
\end{tabular}

a) Individual daily averages are weighted by their 4-year sampling weights as assigned within NHANES 1999-2002 when calculating the statistics in this Table. Inhalation rate was estimated using a multiple linear regression model.

Source: US-EPA, 2009, modified from US-EPA, 2009.

Table C.8. Daily Average Inhalation Rate for Men and Women, by Age Category ${ }^{\mathrm{a}}$ - Adjusted by Body Weight

\begin{tabular}{|c|c|c|c|c|}
\hline \multicolumn{5}{|c|}{ Daily Inhalation Rate - Adjusted for Body Weight } \\
\hline & \multicolumn{2}{|c|}{ Mean $\mathrm{m}^{3} /$ day-kg } & \multicolumn{2}{|c|}{$95^{\text {th }}$ Percentile $\mathrm{m}^{3} /$ day-kg } \\
\hline & Men & Women & Men & Women \\
\hline \multicolumn{5}{|l|}{ Children: } \\
\hline Birth to $<1$ year & 1.09 & 1.14 & 1.29 & 1.38 \\
\hline $1-<2$ years & 1.19 & 1.20 & 1.48 & 1.46 \\
\hline $2-<3$ years & 0.95 & 0.95 & 1.13 & 1.10 \\
\hline $3-<6$ years & 0.70 & 0.69 & 0.92 & 0.92 \\
\hline $6-<11$ years & 0.44 & 0.43 & 0.58 & 0.58 \\
\hline $11-<16$ years & 0.29 & 0.25 & 0.38 & 0.34 \\
\hline $16-<21$ years & 0.23 & 0.21 & 0.30 & 0.28 \\
\hline \multicolumn{5}{|c|}{ Adults (over 21 years): } \\
\hline $21-<31$ years & 0.23 & 0.21 & 0.32 & 0.28 \\
\hline $31-<41$ years & 0.24 & 0.21 & 0.34 & 0.30 \\
\hline $41-<51$ years & 0.24 & 0.22 & 0.34 & 0.31 \\
\hline $51-<61$ years & 0.24 & 0.22 & 0.34 & 0.30 \\
\hline $61-<71$ years & 0.21 & 0.18 & 0.25 & 0.22 \\
\hline $71-<81$ years & 0.20 & 0.18 & 0.24 & 0.23 \\
\hline$\geq 81$ years & 0.20 & 0.18 & 0.25 & 0.22 \\
\hline
\end{tabular}

a) Individual daily averages are weighted by their 4-year sampling weights as assigned within NHANES 1999-2002 when calculating the statistics in this Table. Inhalation rate was estimated using a multiple linear regression model.

Source: US-EPA, 2009, modified from US-EPA, 2009. 
Table C.9. Short Term Exposure Inhalation Rates, by Activity Level - Unadjusted for Body Weight

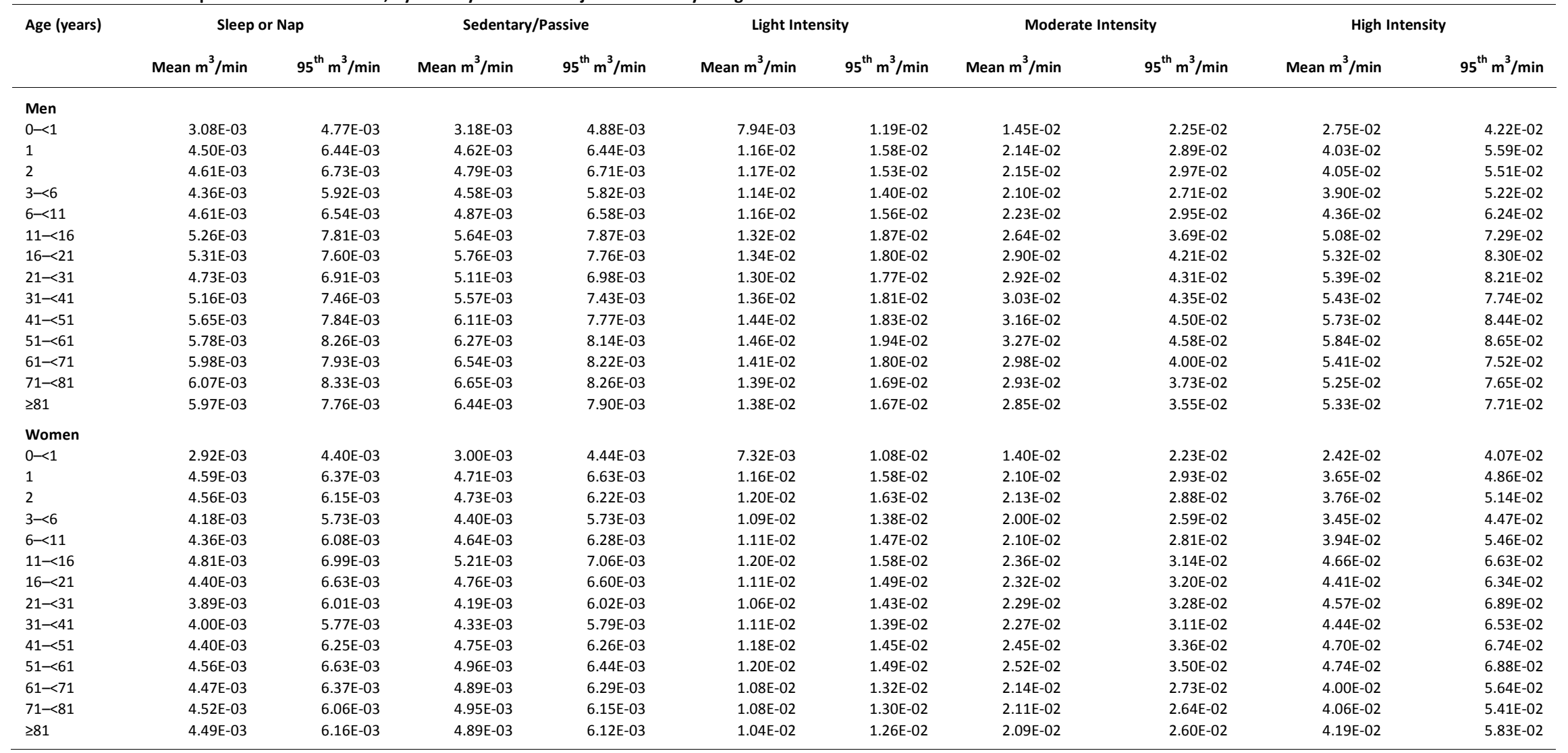

a) An individual's ventilation rate for the given activity category equals the weighted average of the individual's activity-specific ventilation rates for activities falling within the category, estimated using a multiple linear regression model, with weights corresponding to the number of minutes spent performing the activity. Numbers in these two columns represent averages, calculated across individuals in the specified age category, of these weighted averages. These are weighted averages, with the weights corresponding to the 4-year sampling weights assigned within NHANES 1999-2002.

Source: US-EPA, 2009, modified from US-EPA, 2009. 
Table C.10. Short Term Exposure Inhalation Rates, by Activity Level - Adjusted for Body Weight

\begin{tabular}{|c|c|c|c|c|c|c|c|c|c|c|}
\hline \multirow{2}{*}{$\begin{array}{l}\text { Age } \\
\text { (years) }\end{array}$} & \multicolumn{2}{|c|}{ Sleep or Nap } & \multicolumn{2}{|c|}{ Sedentary/Passive } & \multicolumn{2}{|c|}{ Light Intensity } & \multicolumn{2}{|c|}{ Moderate Intensity } & \multicolumn{2}{|c|}{ High Intensity } \\
\hline & Mean $\mathrm{m}^{3} / \mathrm{min}$ & $95^{\text {th }} \mathrm{m}^{3} / \mathrm{min}$ & Mean $\mathrm{m}^{3} / \mathrm{min}$ & $95^{\text {th }} \mathrm{m}^{3} / \mathrm{min}$ & Mean $\mathrm{m}^{3} / \mathrm{min}$ & $95^{\text {th }} \mathrm{m}^{3} / \mathrm{min}$ & Mean $\mathrm{m}^{3} / \mathrm{min}$ & $95^{\text {th }} \mathrm{m}^{3} / \mathrm{min}$ & Mean $\mathrm{m}^{3} / \mathrm{min}$ & $95^{\text {th }} \mathrm{m}^{3} / \mathrm{min}$ \\
\hline \multicolumn{11}{|l|}{ Men } \\
\hline $0-<1$ & $3.85 \mathrm{E}-04$ & $5.03 \mathrm{E}-04$ & 3.97E-04 & $4.98 \mathrm{E}-04$ & $9.88 \mathrm{E}-04$ & $1.20 \mathrm{E}-03$ & $1.80 \mathrm{E}-03$ & $2.18 \mathrm{E}-03$ & $3.48 \mathrm{E}-03$ & 4.14E- 03 \\
\hline 1 & $3.95 \mathrm{E}-04$ & $5.24 \mathrm{E}-04$ & $4.06 \mathrm{E}-04$ & $5.25 \mathrm{E}-04$ & $1.02 \mathrm{E}-03$ & $1.30 \mathrm{E}-03$ & $1.88 \mathrm{E}-03$ & $2.34 \mathrm{E}-03$ & $3.52 \mathrm{E}-03$ & 4.11E-03 \\
\hline 2 & 3.30E- 04 & 4.42E-04 & $3.43 \mathrm{E}-04$ & 4.46E-04 & $8.37 \mathrm{E}-04$ & $1.03 \mathrm{E}-03$ & $1.55 \mathrm{E}-03$ & $1.84 \mathrm{E}-03$ & $2.89 \mathrm{E}-03$ & $3.43 \mathrm{E}-03$ \\
\hline $3-<6$ & $2.43 \mathrm{E}-04$ & $3.50 \mathrm{E}-04$ & $2.55 \mathrm{E}-04$ & $3.46 \mathrm{E}-04$ & $6.33 \mathrm{E}-04$ & $8.71 \mathrm{E}-04$ & $1.17 \mathrm{E}-03$ & $1.56 \mathrm{E}-03$ & 2.17E- 03 & $2.73 \mathrm{E}-03$ \\
\hline $6-<11$ & $1.51 \mathrm{E}-04$ & $2.15 \mathrm{E}-04$ & $1.60 \mathrm{E}-04$ & $2.18 \mathrm{E}-04$ & $3.84 \mathrm{E}-04$ & $5.29 \mathrm{E}-04$ & $7.36 \mathrm{E}-04$ & $9.58 \mathrm{E}-04$ & $1.41 \mathrm{E}-03$ & $1.83 \mathrm{E}-03$ \\
\hline $11-<16$ & $9.80 \mathrm{E}-05$ & $1.41 \mathrm{E}-04$ & $1.05 \mathrm{E}-04$ & $1.42 \mathrm{E}-04$ & $2.46 \mathrm{E}-04$ & $3.32 \mathrm{E}-04$ & $4.91 \mathrm{E}-04$ & $6.35 \mathrm{E}-04$ & 9.50E-04 & $1.27 \mathrm{E}-03$ \\
\hline $16-<21$ & 7.10E-05 & $9.80 \mathrm{E}-05$ & $7.70 \mathrm{E}-05$ & $1.02 \mathrm{E}-04$ & $1.79 \mathrm{E}-04$ & $2.30 \mathrm{E}-04$ & 3.87E-04 & $4.86 \mathrm{E}-04$ & 7.11E-04 & $9.17 \mathrm{E}-04$ \\
\hline $21-<31$ & $5.80 \mathrm{E}-05$ & $8.30 \mathrm{E}-05$ & $6.20 \mathrm{E}-05$ & $8.20 \mathrm{E}-05$ & $1.58 \mathrm{E}-04$ & 2.07E-04 & 3.57E-04 & $4.68 \mathrm{E}-04$ & $6.60 \mathrm{E}-04$ & 8.55E-04 \\
\hline $31-<41$ & $6.10 \mathrm{E}-05$ & $8.60 \mathrm{E}-05$ & $6.60 \mathrm{E}-05$ & $8.60 \mathrm{E}-05$ & $1.61 \mathrm{E}-04$ & $2.09 \mathrm{E}-04$ & 3.57E-04 & $4.71 \mathrm{E}-04$ & $6.44 \mathrm{E}-04$ & $8.53 \mathrm{E}-04$ \\
\hline $41-<51$ & $6.50 \mathrm{E}-05$ & $9.20 \mathrm{E}-05$ & 7.10E-05 & $9.10 \mathrm{E}-05$ & $1.66 \mathrm{E}-04$ & $2.14 \mathrm{E}-04$ & $3.66 \mathrm{E}-04$ & $4.69 \mathrm{E}-04$ & $6.55 \mathrm{E}-04$ & 8.56E-04 \\
\hline $51-<61$ & $6.60 \mathrm{E}-05$ & $9.30 \mathrm{E}-05$ & $7.20 \mathrm{E}-05$ & $9.20 \mathrm{E}-05$ & $1.67 \mathrm{E}-04$ & $2.16 \mathrm{E}-04$ & $3.76 \mathrm{E}-04$ & $4.82 \mathrm{E}-04$ & $6.75 \mathrm{E}-04$ & $9.13 \mathrm{E}-04$ \\
\hline $61-<71$ & $6.90 \mathrm{E}-05$ & $9.30 \mathrm{E}-05$ & $7.60 \mathrm{E}-05$ & $9.40 \mathrm{E}-05$ & $1.64 \mathrm{E}-04$ & $1.95 \mathrm{E}-04$ & $3.44 \mathrm{E}-04$ & 3.99E-04 & $6.24 \mathrm{E}-04$ & $7.88 \mathrm{E}-04$ \\
\hline $71-<81$ & 7.50E-05 & $9.90 \mathrm{E}-05$ & $8.20 \mathrm{E}-05$ & 9.80E-05 & $1.71 \mathrm{E}-04$ & $2.03 E-04$ & 3.60E-04 & $4.18 \mathrm{E}-04$ & 6.46E-04 & $8.49 \mathrm{E}-04$ \\
\hline$\geq 81$ & 8.00E-05 & $1.11 \mathrm{E}-04$ & $8.60 \mathrm{E}-05$ & $1.06 \mathrm{E}-04$ & $1.85 \mathrm{E}-04$ & 2.24E-04 & 3.83E-04 & 4.47E-04 & 7.16E-04 & $9.42 \mathrm{E}-04$ \\
\hline \multicolumn{11}{|l|}{ Women } \\
\hline $0-<1$ & $3.91 \mathrm{E}-04$ & $5.17 \mathrm{E}-04$ & $4.02 \mathrm{E}-04$ & $5.19 \mathrm{E}-04$ & $9.78 \mathrm{E}-04$ & $1.23 \mathrm{E}-03$ & $1.87 \mathrm{E}-03$ & $2.40 \mathrm{E}-03$ & $3.26 \mathrm{E}-03$ & $4.08 \mathrm{E}-03$ \\
\hline 1 & 4.14E- 04 & $5.36 \mathrm{E}-04$ & $4.25 \mathrm{E}-04$ & $5.43 \mathrm{E}-04$ & $1.05 \mathrm{E}-03$ & $1.27 \mathrm{E}-03$ & $1.90 \mathrm{E}-03$ & $2.37 \mathrm{E}-03$ & $3.38 \mathrm{E}-03$ & 4.87E-03 \\
\hline 2 & $3.42 \mathrm{E}-04$ & $4.53 \mathrm{E}-04$ & $3.55 \mathrm{E}-04$ & $4.42 \mathrm{E}-04$ & $8.97 \mathrm{E}-04$ & $1.10 \mathrm{E}-03$ & $1.60 \mathrm{E}-03$ & $2.02 \mathrm{E}-03$ & $2.80 \mathrm{E}-03$ & $3.48 \mathrm{E}-03$ \\
\hline $11-<16$ & $9.00 \mathrm{E}-05$ & $1.30 \mathrm{E}-04$ & $9.70 \mathrm{E}-05$ & $1.33 \mathrm{E}-04$ & $2.25 \mathrm{E}-04$ & $3.05 E-04$ & $4.41 \mathrm{E}-04$ & $6.11 \mathrm{E}-04$ & $8.79 \mathrm{E}-04$ & $1.31 \mathrm{E}-03$ \\
\hline $16-<21$ & $6.90 \mathrm{E}-05$ & $1.02 \mathrm{E}-04$ & $7.50 \mathrm{E}-05$ & $1.04 \mathrm{E}-04$ & $1.74 \mathrm{E}-04$ & $2.24 \mathrm{E}-04$ & $3.65 \mathrm{E}-04$ & 4.94E-04 & 6.96E-04 & 1.00E-03 \\
\hline $21-<31$ & $5.50 \mathrm{E}-05$ & $8.20 \mathrm{E}-05$ & $6.00 \mathrm{E}-05$ & $8.00 \mathrm{E}-05$ & $1.49 \mathrm{E}-04$ & $1.90 \mathrm{E}-04$ & $3.25 \mathrm{E}-04$ & $4.52 \mathrm{E}-04$ & $6.50 \mathrm{E}-04$ & $9.39 \mathrm{E}-04$ \\
\hline $31-<41$ & $5.60 \mathrm{E}-05$ & $8.20 \mathrm{E}-05$ & $6.00 \mathrm{E}-05$ & $8.30 \mathrm{E}-05$ & $1.54 \mathrm{E}-04$ & $2.02 \mathrm{E}-04$ & $3.16 \mathrm{E}-04$ & $4.60 \mathrm{E}-04$ & $6.13 \mathrm{E}-04$ & $9.05 \mathrm{E}-04$ \\
\hline $41-<51$ & $6.00 \mathrm{E}-05$ & $9.00 \mathrm{E}-05$ & $6.50 \mathrm{E}-05$ & $9.10 \mathrm{E}-05$ & $1.61 \mathrm{E}-04$ & $2.16 \mathrm{E}-04$ & $3.33 \mathrm{E}-04$ & $4.88 \mathrm{E}-04$ & $6.35 \mathrm{E}-04$ & 9.50E-04 \\
\hline $51-<61$ & $6.10 \mathrm{E}-05$ & $8.80 \mathrm{E}-05$ & $6.70 \mathrm{E}-05$ & $9.00 \mathrm{E}-05$ & $1.61 \mathrm{E}-04$ & $2.10 \mathrm{E}-04$ & $3.39 \mathrm{E}-04$ & $4.86 \mathrm{E}-04$ & $6.34 \mathrm{E}-04$ & $9.28 \mathrm{E}-04$ \\
\hline $61-<71$ & $6.10 \mathrm{E}-05$ & $8.10 \mathrm{E}-05$ & $6.60 \mathrm{E}-05$ & $8.40 \mathrm{E}-05$ & $1.47 \mathrm{E}-04$ & $1.82 \mathrm{E}-04$ & $2.92 \mathrm{E}-04$ & $3.71 \mathrm{E}-04$ & $5.44 \mathrm{E}-04$ & $8.03 \mathrm{E}-04$ \\
\hline $71-<81$ & $6.60 \mathrm{E}-05$ & $9.00 \mathrm{E}-05$ & $7.20 \mathrm{E}-05$ & $9.20 \mathrm{E}-05$ & $1.58 \mathrm{E}-04$ & $2.02 E-04$ & $3.08 \mathrm{E}-04$ & 4.07E-04 & $5.94 \mathrm{E}-04$ & $8.29 \mathrm{E}-04$ \\
\hline$\geq 81$ & $7.20 \mathrm{E}-05$ & $9.60 \mathrm{E}-05$ & $7.80 \mathrm{E}-05$ & $9.60 \mathrm{E}-05$ & $1.67 \mathrm{E}-04$ & $2.08 \mathrm{E}-04$ & $3.35 \mathrm{E}-04$ & $4.20 \mathrm{E}-04$ & $6.66 \mathrm{E}-04$ & $9.72 \mathrm{E}-04$ \\
\hline
\end{tabular}

a) An individual's ventilation rate for the given activity category equals the weighted average of the individual's activity-specific ventilation rates for activities falling within the category, estimated using a multiple linear regression model, with weights corresponding to the number of minutes spent performing the activity. Numbers in these two columns represent averages, calculated across individuals in the specified age category, of these weighted averages. These are weighted averages, with the weights corresponding to the 4-year sampling weights assigned within NHANES 1999-2002.

Source: US-EPA, 2009, modified from US-EPA, 2009. 


\section{Annex D.}

\section{Drinking Water - Supplementary Tables}

\begin{tabular}{lrr} 
Table D.1. Plain Tap Water and Total Water Consumption by Age and Sex \\
\hline Variable & Plain Tap Water (mL/kg-day) Mean & Total Water (mL/kg-day) Mean \\
\hline Infants & 11 & 130 \\
$<12$ months & 18 & 108 \\
$12-24$ months & & 116 \\
Sex & 15 & 119 \\
Men & 15 & \\
Women & & \\
\hline
\end{tabular}

Source: Heller et al., 2000, modified from US-EPA, 2009. 


\section{Annex E. Food Intake - Supplementary Tables}

Table E.1. Dietary Habits in Denmark 2003-2008

\begin{tabular}{|c|c|c|}
\hline Group (years) & Mean & $90^{\text {th }}$ percentiles \\
\hline \multicolumn{3}{|c|}{ Cheese and cheese products (g/day) } \\
\hline Boys 4-5 & 16 & 31 \\
\hline Boys $6-9$ & 22 & 47 \\
\hline Boys 4-9 & 20 & 41 \\
\hline Boys $10-13$ & 27 & 54 \\
\hline Boys $14-17$ & 34 & 56 \\
\hline Boys $10-17$ & 30 & 59 \\
\hline Men 18-24 & 48 & 95 \\
\hline Men 25-34 & 40 & 73 \\
\hline Men 35-44 & 39 & 72 \\
\hline Men 45-54 & 41 & 78 \\
\hline Men 55-64 & 37 & 71 \\
\hline Men 65-75 & 32 & 62 \\
\hline Men 18-75 & 39 & 74 \\
\hline Total (boys and men) & 35 & 70 \\
\hline Girls 4-5 & 16 & 31 \\
\hline Girls 6-9 & 17 & 34 \\
\hline Girls 4-9 & 17 & 32 \\
\hline Girls $10-13$ & 23 & 45 \\
\hline Girls $14-17$ & 22 & 47 \\
\hline Girls $10-17$ & 23 & 45 \\
\hline Women $18-24$ & 29 & 51 \\
\hline Women 25-34 & 30 & 26 \\
\hline Women 35-44 & 34 & 62 \\
\hline Women 45-54 & 32 & 57 \\
\hline Women 55-64 & 29 & 55 \\
\hline Women 65-75 & 25 & 47 \\
\hline Women 18-75 & 30 & 54 \\
\hline Total (girls and women) & 28 & 56 \\
\hline All children 4-9 & 19 & 37 \\
\hline All children $10-17$ & 26 & 52 \\
\hline All adults $18-75$ & 34 & 65 \\
\hline Total (all) & 31 & 62 \\
\hline \multicolumn{3}{|c|}{ Cereals including bread (g/day) } \\
\hline Boys $4-5$ & 193 & 248 \\
\hline Boys $6-9$ & 220 & 310 \\
\hline Boys 4-9 & 211 & 290 \\
\hline Boys $10-13$ & 236 & 334 \\
\hline Boys $14-17$ & 242 & 350 \\
\hline Boys $10-17$ & 238 & 334 \\
\hline Men 18-24 & 252 & 373 \\
\hline Men 25-34 & 259 & 362 \\
\hline Men 35-44 & 264 & 377 \\
\hline Men 45-54 & 245 & 368 \\
\hline Men 55-64 & 229 & 332 \\
\hline Men 65-75 & 223 & 328 \\
\hline Men 18-75 & 245 & 356 \\
\hline Total (boys and men) & 240 & 349 \\
\hline
\end{tabular}




\begin{tabular}{|c|c|c|}
\hline Group (years) & Mean & $90^{\text {th }}$ percentiles \\
\hline Girls 4-5 & 170 & 227 \\
\hline Girls 6-9 & 193 & 262 \\
\hline Girls 4-9 & 186 & 249 \\
\hline Girls $10-13$ & 194 & 266 \\
\hline Girls $14-17$ & 185 & 282 \\
\hline Girls $10-17$ & 190 & 270 \\
\hline Women 18-24 & 187 & 289 \\
\hline Women 25-34 & 196 & 274 \\
\hline Women 35-44 & 204 & 295 \\
\hline Women 45-54 & 180 & 267 \\
\hline Women 55-64 & 173 & 254 \\
\hline Women 65-75 & 171 & 250 \\
\hline Women 18-75 & 187 & 273 \\
\hline Total (girls and women) & 187 & 272 \\
\hline All children 4-9 & 199 & 276 \\
\hline All children 10-17 & 212 & 298 \\
\hline All adults $18-75$ & 214 & 320 \\
\hline Total (all) & 212 & 314 \\
\hline \multicolumn{3}{|c|}{ Vegetables exclusive potatoes (g/day) } \\
\hline Boys 4-5 & 113 & 201 \\
\hline Boys $6-9$ & 134 & 230 \\
\hline Boys 4-9 & 127 & 219 \\
\hline Boys $10-13$ & 137 & 257 \\
\hline Boys $14-17$ & 137 & 238 \\
\hline Boys $10-17$ & 137 & 251 \\
\hline Men 18-24 & 158 & 251 \\
\hline Men 25-34 & 170 & 285 \\
\hline Men 35-44 & 152 & 254 \\
\hline Men 45-54 & 153 & 251 \\
\hline Men 55-64 & 145 & 249 \\
\hline Men 65-75 & 125 & 223 \\
\hline Men 18-75 & 150 & 253 \\
\hline Total (boys and men) & 145 & 249 \\
\hline Girls 4-5 & 117 & 205 \\
\hline Girls 6-9 & 115 & 201 \\
\hline Girls 4-9 & 116 & 205 \\
\hline Girls $10-13$ & 126 & 220 \\
\hline Girls $14-17$ & 127 & 215 \\
\hline Girls $10-17$ & 126 & 217 \\
\hline Women 18-24 & 172 & 292 \\
\hline Women 25-34 & 182 & 307 \\
\hline Women 35-44 & 190 & 343 \\
\hline Women $45-54$ & 168 & 277 \\
\hline Women 55-64 & 166 & 293 \\
\hline Women $65-75$ & 136 & 252 \\
\hline Women $18-75$ & 172 & 297 \\
\hline Total (girls and women) & 160 & 280 \\
\hline All children 4-9 & 122 & 207 \\
\hline All children $10-17$ & 131 & 230 \\
\hline All adults $18-75$ & 162 & 278 \\
\hline Total (all) & 153 & 265 \\
\hline \multicolumn{3}{|c|}{ Potatoes and potato products (g/day) } \\
\hline Boys $4-5$ & 57 & 109 \\
\hline Boys 6-9 & 64 & 133 \\
\hline Boys 4-9 & 74 & 140 \\
\hline Boys $10-13$ & 84 & 176 \\
\hline Boys $14-17$ & 111 & 222 \\
\hline Boys $10-17$ & 101 & 215 \\
\hline Men 18-24 & 113 & 240 \\
\hline Men 25-34 & 111 & 225 \\
\hline Men 35-44 & 114 & 218 \\
\hline Men 45-54 & 123 & 241 \\
\hline Men 55-64 & 144 & 270 \\
\hline Men 65-75 & 149 & 263 \\
\hline Men 18-75 & 126 & 242 \\
\hline Total (boys and men) & 115 & 231 \\
\hline
\end{tabular}




\begin{tabular}{|c|c|c|}
\hline Group (years) & Mean & $90^{\text {th }}$ percentiles \\
\hline Girls 4-5 & 45 & 104 \\
\hline Girls 6-9 & 59 & 114 \\
\hline Girls 4-9 & 73 & 137 \\
\hline Girls $10-13$ & 65 & 147 \\
\hline Girls $14-17$ & 70 & 141 \\
\hline Girls $10-17$ & 91 & 184 \\
\hline Women 18-24 & 67 & 126 \\
\hline Women 25-34 & 63 & 125 \\
\hline Women 35-44 & 68 & 126 \\
\hline Women 45-54 & 81 & 158 \\
\hline Women 55-64 & 91 & 172 \\
\hline Women 65-75 & 107 & 182 \\
\hline Women 18-75 & 100 & 196 \\
\hline Total (girls and women) & 74 & 146 \\
\hline All children 4-9 & 74 & 139 \\
\hline All children 10-17 & 95 & 198 \\
\hline All adults $18-75$ & 112 & 219 \\
\hline Total (all) & 94 & 189 \\
\hline \multicolumn{3}{|c|}{ Fruits and fruit products (g/day) } \\
\hline Boys 4-5 & 197 & 312 \\
\hline Boys $6-9$ & 189 & 382 \\
\hline Boys 4-9 & 274 & 553 \\
\hline Boys $10-13$ & 176 & 410 \\
\hline Boys $14-17$ & 136 & 301 \\
\hline Boys $10-17$ & 234 & 539 \\
\hline Men 18-24 & 104 & 242 \\
\hline Men 25-34 & 186 & 405 \\
\hline Men 35-44 & 160 & 333 \\
\hline Men 45-54 & 196 & 393 \\
\hline Men 55-64 & 195 & 422 \\
\hline Men 65-75 & 201 & 389 \\
\hline Men 18-75 & 251 & 511 \\
\hline Total (boys and men) & 180 & 379 \\
\hline Girls 4-5 & 173 & 296 \\
\hline Girls 6-9 & 175 & 302 \\
\hline Girls 4-9 & 245 & 456 \\
\hline Girls $10-13$ & 177 & 343 \\
\hline Girls $14-17$ & 168 & 358 \\
\hline Girls $10-17$ & 282 & 541 \\
\hline Women $18-24$ & 187 & 354 \\
\hline Women 25-34 & 228 & 437 \\
\hline Women 35-44 & 227 & 436 \\
\hline Women 45-54 & 241 & 447 \\
\hline Women 55-64 & 280 & 510 \\
\hline Women 65-75 & 241 & 431 \\
\hline Women 18-75 & 311 & 575 \\
\hline Total (girls and women) & 222 & 431 \\
\hline All children 4-9 & 260 & 469 \\
\hline All children $10-17$ & 274 & 541 \\
\hline All adults $18-75$ & 283 & 548 \\
\hline Total (all) & 280 & 541 \\
\hline \multicolumn{3}{|c|}{ Meat and meat products (g/day) } \\
\hline Boys $4-5$ & 81 & 117 \\
\hline Boys 6-9 & 94 & 139 \\
\hline Boys 4-9 & 90 & 134 \\
\hline Boys $10-13$ & 112 & 173 \\
\hline Boys $14-17$ & 132 & 212 \\
\hline Boys $10-17$ & 119 & 192 \\
\hline Men 18-24 & 147 & 224 \\
\hline Men 25-34 & 151 & 234 \\
\hline Men 35-44 & 152 & 261 \\
\hline Men $45-54$ & 140 & 223 \\
\hline Men 55-64 & 135 & 223 \\
\hline Men 65-75 & 115 & 178 \\
\hline Men 18-75 & 140 & 225 \\
\hline Total (boys and men) & 131 & 213 \\
\hline
\end{tabular}




\begin{tabular}{|c|c|c|}
\hline Group (years) & Mean & $90^{\text {th }}$ percentiles \\
\hline Girls 4-5 & 67 & 102 \\
\hline Girls 6-9 & 81 & 121 \\
\hline Girls 4-9 & 76 & 117 \\
\hline Girls $10-13$ & 83 & 128 \\
\hline Girls $14-17$ & 73 & 126 \\
\hline Girls $10-17$ & 79 & 127 \\
\hline Women $18-24$ & 78 & 127 \\
\hline Women 25-34 & 82 & 128 \\
\hline Women 35-44 & 87 & 136 \\
\hline Women $45-54$ & 84 & 140 \\
\hline Women 55-64 & 80 & 125 \\
\hline Women 65-75 & 75 & 123 \\
\hline Women 18-75 & 82 & 133 \\
\hline Total (girls and women) & 81 & 130 \\
\hline All children 4-9 & 83 & 126 \\
\hline All children 10-17 & 97 & 162 \\
\hline All adults $18-75$ & 109 & 188 \\
\hline Total (all) & 105 & 179 \\
\hline \multicolumn{3}{|c|}{ Poultry and poultry products (g/day) } \\
\hline Boys 4-5 & 14 & 35 \\
\hline Boys 6-9 & 17 & 40 \\
\hline Boys 4-9 & 16 & 39 \\
\hline Boys $10-13$ & 23 & 56 \\
\hline Boys $14-17$ & 23 & 58 \\
\hline Boys $10-17$ & 23 & 56 \\
\hline Men 18-24 & 33 & 77 \\
\hline Men 25-34 & 32 & 74 \\
\hline Men 35-44 & 28 & 66 \\
\hline Men 45-54 & 25 & 59 \\
\hline Men 55-64 & 20 & 50 \\
\hline Men 65-75 & 20 & 56 \\
\hline Men 18-75 & 26 & 62 \\
\hline Total (boys and men) & 24 & 58 \\
\hline Girls 4-5 & 13 & 30 \\
\hline Girls 6-9 & 17 & 39 \\
\hline Girls 4-9 & 16 & 37 \\
\hline Girls $10-13$ & 21 & 46 \\
\hline Girls $14-17$ & 22 & 51 \\
\hline Girls $10-17$ & 21 & 47 \\
\hline Women $18-24$ & 23 & 52 \\
\hline Women 25-34 & 24 & 53 \\
\hline Women 35-44 & 23 & 48 \\
\hline Women $45-54$ & 21 & 47 \\
\hline Women 55-64 & 18 & 46 \\
\hline Women 65-75 & 20 & 42 \\
\hline Women 18-75 & 21 & 49 \\
\hline Total (girls and women) & 21 & 47 \\
\hline All children 4-9 & 16 & 37 \\
\hline All children 10-17 & 22 & 51 \\
\hline All adults $18-75$ & 23 & 55 \\
\hline Total (all) & 22 & 53 \\
\hline \multicolumn{3}{|c|}{ Fish and fish products (g/day) } \\
\hline Boys $4-5$ & 13 & 30 \\
\hline Boys 6-9 & 13 & 32 \\
\hline Boys 4-9 & 13 & 32 \\
\hline Boys $10-13$ & 13 & 33 \\
\hline Boys $14-17$ & 14 & 31 \\
\hline Boys $10-17$ & 13 & 32 \\
\hline Men $18-24$ & 14 & 35 \\
\hline Men 25-34 & 18 & 42 \\
\hline Men 35-44 & 21 & 49 \\
\hline Men 45-54 & 23 & 49 \\
\hline Men 55-64 & 30 & 63 \\
\hline Men $65-75$ & 26 & 54 \\
\hline Men 18-75 & 23 & 51 \\
\hline Total (boys and men) & 21 & 46 \\
\hline
\end{tabular}




\begin{tabular}{|c|c|c|}
\hline Group (years) & Mean & $90^{\text {th }}$ percentiles \\
\hline Girls 4-5 & 12 & 32 \\
\hline Girls 6-9 & 11 & 28 \\
\hline Girls 4-9 & 11 & 30 \\
\hline Girls $10-13$ & 10 & 23 \\
\hline Girls 14-17 & 10 & 24 \\
\hline Girls 10-17 & 10 & 23 \\
\hline Women 18-24 & 16 & 38 \\
\hline Women 25-34 & 18 & 39 \\
\hline Women 35-44 & 19 & 45 \\
\hline Women 45-54 & 20 & 43 \\
\hline Women 55-64 & 23 & 50 \\
\hline Women $65-75$ & 25 & 52 \\
\hline Women 18-75 & 20 & 46 \\
\hline Total (girls and women) & 18 & 42 \\
\hline All children 4-9 & 12 & 31 \\
\hline All children 10-17 & 11 & 28 \\
\hline All adults $18-75$ & 22 & 47 \\
\hline Total (all) & 19 & 44 \\
\hline \multicolumn{3}{|l|}{ Eggs (g/day) } \\
\hline Boys 4-5 & 13 & 22 \\
\hline Boys 6-9 & 13 & 26 \\
\hline Boys 4-9 & 13 & 26 \\
\hline Boys $10-13$ & 14 & 31 \\
\hline Boys $14-17$ & 12 & 27 \\
\hline Boys $10-17$ & 14 & 30 \\
\hline Men 18-24 & 13 & 31 \\
\hline Men 25-34 & 16 & 37 \\
\hline Men 35-44 & 18 & 37 \\
\hline Men 45-54 & 18 & 36 \\
\hline Men 55-64 & 22 & 45 \\
\hline Men 65-75 & 22 & 48 \\
\hline Men 18-75 & 19 & 40 \\
\hline Total (boys and men) & 17 & 36 \\
\hline Girls 4-5 & 12 & 23 \\
\hline Girls 6-9 & 12 & 27 \\
\hline Girls 4-9 & 12 & 26 \\
\hline Girls $10-13$ & 12 & 24 \\
\hline Girls 14-17 & 11 & 23 \\
\hline Girls $10-17$ & 11 & 24 \\
\hline Women 18-24 & 14 & 32 \\
\hline Women 25-34 & 16 & 34 \\
\hline Women 35-44 & 15 & 30 \\
\hline Women $45-54$ & 15 & 32 \\
\hline Women 55-64 & 17 & 32 \\
\hline Women $65-75$ & 17 & 34 \\
\hline Women 18-75 & 16 & 32 \\
\hline Total (girls and women) & 15 & 31 \\
\hline All children 4-9 & 13 & 26 \\
\hline All children $10-17$ & 12 & 28 \\
\hline All adults $18-75$ & 17 & 35 \\
\hline Total (all) & 16 & 33 \\
\hline \multicolumn{3}{|l|}{ Fats (g/day) } \\
\hline Boys $4-5$ & 34 & 53 \\
\hline Boys 6-9 & 35 & 57 \\
\hline Boys 4-9 & 35 & 55 \\
\hline Boys $10-13$ & 33 & 56 \\
\hline Boys $14-17$ & 35 & 59 \\
\hline Boys $10-17$ & 34 & 57 \\
\hline Men 18-24 & 39 & 60 \\
\hline Men 25-34 & 43 & 71 \\
\hline Men 35-44 & 50 & 87 \\
\hline Men 45-54 & 42 & 77 \\
\hline Men 55-64 & 40 & 73 \\
\hline Men 65-75 & 41 & 75 \\
\hline Men 18-75 & 43 & 76 \\
\hline Total (boys and men) & 41 & 71 \\
\hline
\end{tabular}




\begin{tabular}{|c|c|c|}
\hline Group (years) & Mean & $90^{\text {th }}$ percentiles \\
\hline Girls 4-5 & 30 & 53 \\
\hline Girls 6-9 & 32 & 53 \\
\hline Girls 4-9 & 32 & 53 \\
\hline Girls $10-13$ & 28 & 48 \\
\hline Girls $14-17$ & 25 & 41 \\
\hline Girls $10-17$ & 27 & 46 \\
\hline Women 18-24 & 29 & 49 \\
\hline Women 25-34 & 31 & 54 \\
\hline Women 35-44 & 32 & 52 \\
\hline Women 45-54 & 27 & 45 \\
\hline Women 55-64 & 26 & 45 \\
\hline Women 65-75 & 28 & 48 \\
\hline Women 18-75 & 29 & 50 \\
\hline Total (girls and women) & 29 & 49 \\
\hline All children 4-9 & 33 & 55 \\
\hline All children 10-17 & 30 & 52 \\
\hline All adults $18-75$ & 35 & 63 \\
\hline Total (all) & 34 & 60 \\
\hline \multicolumn{3}{|l|}{ Sugar and candy (g/day) } \\
\hline Boys 4-5 & 35 & 62 \\
\hline Boys 6-9 & 40 & 72 \\
\hline Boys 4-9 & 38 & 69 \\
\hline Boys $10-13$ & 43 & 82 \\
\hline Boys $14-17$ & 41 & 93 \\
\hline Boys $10-17$ & 42 & 85 \\
\hline Men 18-24 & 32 & 73 \\
\hline Men 25-34 & 42 & 85 \\
\hline Men 35-44 & 42 & 84 \\
\hline Men 45-54 & 33 & 73 \\
\hline Men 55-64 & 29 & 64 \\
\hline Men 65-75 & 30 & 61 \\
\hline Men 18-75 & 35 & 74 \\
\hline Total (boys and men) & 36 & 75 \\
\hline Girls 4-5 & 35 & 63 \\
\hline Girls 6-9 & 41 & 64 \\
\hline Girls 4-9 & 39 & 64 \\
\hline Girls $10-13$ & 45 & 80 \\
\hline Girls $14-17$ & 38 & 63 \\
\hline Girls $10-17$ & 42 & 74 \\
\hline Women $18-24$ & 39 & 68 \\
\hline Women 25-34 & 39 & 74 \\
\hline Women 35-44 & 38 & 68 \\
\hline Women $45-54$ & 29 & 60 \\
\hline Women 55-64 & 25 & 51 \\
\hline Women 65-75 & 28 & 59 \\
\hline Women $18-75$ & 33 & 64 \\
\hline Total (girls and women) & 35 & 66 \\
\hline All children 4-9 & 39 & 67 \\
\hline All children 10-17 & 42 & 78 \\
\hline All adults $18-75$ & 34 & 69 \\
\hline Total (all) & 36 & 70 \\
\hline \multicolumn{3}{|l|}{ Energy (MJ/day) } \\
\hline Boys $4-5$ & 7.7 & 9.4 \\
\hline Boys 6-9 & 8.8 & 11.3 \\
\hline Boys 4-9 & 8.5 & 11.0 \\
\hline Boys $10-13$ & 9.3 & 11.8 \\
\hline Boys $14-17$ & 10.1 & 13.4 \\
\hline Boys $10-17$ & 9.6 & 12.5 \\
\hline Men 18-24 & 11.1 & 15.4 \\
\hline Men 25-34 & 11.3 & 14.8 \\
\hline Men 35-44 & 11.1 & 15.0 \\
\hline Men 45-54 & 10.3 & 13.8 \\
\hline Men 55-64 & 9.9 & 13.4 \\
\hline Men 65-75 & 9.5 & 13.0 \\
\hline Men 18-75 & 10.4 & 14.3 \\
\hline Total (boys and men) & 10.1 & 13.8 \\
\hline
\end{tabular}




\begin{tabular}{lrr}
\hline Group (years) & Mean & 90 $^{\text {th }}$ percentiles \\
\hline Girls 4-5 & 7.0 & 8.8 \\
Girls 6-9 & 7.8 & 9.8 \\
Girls 4-9 & 7.5 & 9.7 \\
Girls 10-13 & 7.9 & 10.5 \\
Girls 14-17 & 7.4 & 10.5 \\
Girls 10-17 & 7.7 & 10.5 \\
Women 18-24 & 8.2 & 11.0 \\
Women 25-34 & 8.3 & 11.1 \\
Women 35-44 & 8.3 & 11.1 \\
Women 45-54 & 7.6 & 9.8 \\
Women 55-64 & 7.5 & 9.9 \\
Women 65-75 & 7.4 & 9.7 \\
Women 18-75 & 7.9 & 10.5 \\
Total (girls and women) & 7.9 & 10.4 \\
All children 4-9 & 8.0 & 10.6 \\
All children 10-17 & 8.6 & 11.6 \\
All adults 18-75 & 9.1 & 12.9 \\
Total (all) & 8.9 & 12.5 \\
\hline
\end{tabular}

Source: Modified from Dietary habits in Denmark 2003-2008 - Main Results, National Food Institute, Technical University of Denmark, (2010). 


\section{Annex F.}

\section{Soil and Dust ingestion - Supplementary Tables}

\begin{tabular}{|c|c|c|c|c|}
\hline Age (years) & Ingestion medium & Mean & P95 & Reference \\
\hline $0.1-<1$ & Soil & $0-30^{a}$ & - & Van Wijnen et al., 1990 \\
\hline $1-<5$ & Soil & $0-200^{a}$ & - & \\
\hline \multirow[t]{2}{*}{$2-<8$} & Soil & $39-246$ & - & Davis et al., 1990 \\
\hline & Soil and dust & $65-268$ & - & \\
\hline \multirow[t]{3}{*}{$1-<4$} & Soil & $-294-+459$ & $106-1,903$ & Calabrese et al., 1989 \\
\hline & Dust & $-1,289-+964$ & $160-2,916$ & \\
\hline & Soil and dust & $-496-+483$ & $159-3,174$ & \\
\hline Adult & Soil & $23-625$ & $138-2,899$ & Davis and Mirick, 2006 \\
\hline $3-<8$ & Soil & $37-207$ & $95-808$ & Davis and Mirick, 2006 \\
\hline $1-<4$ & Soil & $-544-+270$ & $69-1,378$ & Calabrese et al., 1997a \\
\hline$<1-<7$ & Soil and dust & 113 & - & Hogan et al., 1998 \\
\hline Adult & Soil & $50,000^{b}$ & - & Vermeer and Frate, 1979 \\
\hline $1-13+$ & Soil & $50,000^{b}$ & - & Vermeer and Frate, 1979 \\
\hline $0.3-14$ & Soil & - & $\sim 4,000$ & Wong, 1988/Calabrese and Stanek, 1993 \\
\hline
\end{tabular}

a) Geometric mean

b) Average includes adults and children.

Source: Modified from US-EPA, 2009. 


\section{Annex G.}

\section{Life Expectancy - Supplementary Tables}

\begin{tabular}{|c|c|c|c|}
\hline \multirow[t]{2}{*}{ Country } & \multicolumn{2}{|c|}{ Life Expectancy in Years } & \multirow[b]{2}{*}{ Men and Women Combined } \\
\hline & Men & Women & \\
\hline Albania & 70 & 76 & 73 \\
\hline Armenia & 67 & 74 & 71 \\
\hline Austria & 74 & 80 & 77 \\
\hline Azerbaijan & 66 & 74 & 70 \\
\hline Belarus & 62 & 74 & 68 \\
\hline Belgium & 74 & 81 & 78 \\
\hline Bosnia and Herzegovina & 71 & 76 & 74 \\
\hline Bulgaria & 68 & 75 & 72 \\
\hline Croatia & 69 & 77 & 73 \\
\hline Czech Republic & 70 & 77 & 74 \\
\hline Denmark & 73 & 78 & 76 \\
\hline Estonia & 63 & 75 & 69 \\
\hline Finland & 73 & 81 & 77 \\
\hline France & 74 & 82 & 78 \\
\hline Georgia & 69 & 77 & 73 \\
\hline Germany & 74 & 80 & 77 \\
\hline Greece & 76 & 81 & 79 \\
\hline Hungary & 67 & 75 & 71 \\
\hline Iceland & 77 & 81 & 79 \\
\hline Ireland & 74 & 79 & 77 \\
\hline Israel & 76 & 80 & 78 \\
\hline Italy & 75 & 81 & 78 \\
\hline Kazakhstan & 63 & 72 & 68 \\
\hline Kyrgyzstan & 63 & 72 & 68 \\
\hline Latvia & 62 & 74 & 68 \\
\hline Lithuania & 64 & 76 & 70 \\
\hline Luxembourg & 73 & 80 & 77 \\
\hline Malta & 75 & 79 & 77 \\
\hline Netherlands & 75 & 81 & 78 \\
\hline Norway & 75 & 81 & 78 \\
\hline Poland & 68 & 77 & 73 \\
\hline Portugal & 72 & 79 & 76 \\
\hline Republic of Moldova & 64 & 72 & 68 \\
\hline Romania & 66 & 74 & 70 \\
\hline Russian Federation & 61 & 73 & 67 \\
\hline Slovakia & 69 & 77 & 73 \\
\hline Slovenia & 71 & 78 & 75 \\
\hline Spain & 75 & 82 & 79 \\
\hline Sweden & 76 & 81 & 79 \\
\hline Switzerland & 75 & 82 & 79 \\
\hline Tajikistan & 64 & 70 & 67 \\
\hline The Former Yugoslav Republic of Macedonia & 71 & 75 & 73 \\
\hline Turkey & 67 & 72 & 70 \\
\hline Turkmenistan & 62 & 69 & 66 \\
\hline Ukraine & 64 & 74 & 69 \\
\hline United Kingdom & 75 & 80 & 78 \\
\hline Uzbekistan & 64 & 71 & 68 \\
\hline Yugoslavia & 70 & 75 & 73 \\
\hline
\end{tabular}

Source: WHO, 1999b; modified from ECETOC, 2001 
Table G.2. Expectation of Life at Birth (in 1996) and from Selected Ages in England

\begin{tabular}{lrrrrrr}
\hline Age (years) & $\mathbf{1 9 8 2}$ & $\mathbf{1 9 9 2}$ & $\mathbf{1 9 9 3}$ & $\mathbf{1 9 9 4}$ & $\mathbf{1 9 9 5}$ & $\mathbf{1 9 9 6}$ \\
\hline Men & & & & & & \\
Birth (0) & 71.3 & 73.7 & 74.0 & 74.1 & 74.4 & 74.6 \\
5 & 67.3 & 69.3 & 69.6 & 69.7 & 70.0 & 70.2 \\
20 & 52.7 & 54.6 & 54.9 & 55.0 & 55.3 & 55.5 \\
30 & 43.1 & 45.0 & 45.3 & 45.5 & 45.7 & 45.9 \\
50 & 24.5 & 26.3 & 26.6 & 26.7 & 27.0 & 27.1 \\
60 & 16.5 & 18.0 & 18.2 & 18.3 & 18.5 & 18.7 \\
70 & 10.2 & 11.2 & 11.4 & 11.4 & 11.6 & 11.7 \\
80 & 5.8 & 6.4 & 6.5 & 6.6 & 6.6 & 6.7 \\
Women & & & & & 79.6 & 79.7 \\
Birth (0) & 77.3 & 79.1 & 79.3 & 79.4 & 75.1 & 75.2 \\
5 & 73.1 & 74.6 & 74.9 & 74.9 & 60.3 & 60.3 \\
20 & 58.3 & 59.8 & 60.0 & 60.1 & 50.5 & 50.5 \\
30 & 48.5 & 50.0 & 50.2 & 50.3 & 31.4 & 31.4 \\
50 & 29.6 & 30.9 & 31.1 & 31.2 & 22.5 & 22.6 \\
60 & 21.0 & 22.1 & 22.3 & 22.4 & 14.7 & 14.7 \\
70 & 13.5 & 14.5 & 14.6 & 14.6 & 8.6 & 8.6 \\
80 & 7.6 & 8.4 & 8.5 & 8.5 & & \\
\hline
\end{tabular}

Source: UK Government Actuary's Department, 1998, modified from ECETOC, 2001

Table G.3. Average Life Expectancy at Birth in 2008 According to WHO, European Countries

\begin{tabular}{|c|c|c|c|}
\hline \multirow[t]{2}{*}{ Country } & \multicolumn{3}{|c|}{ Life Expectancy in Years } \\
\hline & Men & Women & Men and Women Combined \\
\hline Albania & 71 & 74 & 73 \\
\hline Armenia & 66 & 73 & 70 \\
\hline Austria & 78 & 83 & 80 \\
\hline Azerbaijan & 66 & 70 & 68 \\
\hline Belarus & 64 & 76 & 70 \\
\hline Belgium & 77 & 82 & 80 \\
\hline Bosnia and Herzegovina & 73 & 78 & 75 \\
\hline Bulgaria & 70 & 77 & 73 \\
\hline Croatia & 72 & 79 & 76 \\
\hline Czech Republic & 74 & 80 & 77 \\
\hline Denmark & 77 & 81 & 79 \\
\hline Estonia & 69 & 79 & 74 \\
\hline Finland & 76 & 83 & 80 \\
\hline France & 78 & 85 & 81 \\
\hline Georgia & 67 & 76 & 72 \\
\hline Germany & 77 & 83 & 80 \\
\hline Greece & 78 & 83 & 80 \\
\hline Hungary & 70 & 78 & 74 \\
\hline Iceland & 80 & 83 & 82 \\
\hline Ireland & 78 & 82 & 80 \\
\hline Israel & 79 & 83 & 81 \\
\hline Italy & 79 & 84 & 82 \\
\hline Kazakhstan & 59 & 70 & 64 \\
\hline Kyrgyzstan & 62 & 69 & 66 \\
\hline Latvia & 66 & 77 & 71 \\
\hline Lithuania & 66 & 78 & 72 \\
\hline Luxembourg & 77 & 83 & 80 \\
\hline Malta & 78 & 82 & 80 \\
\hline Netherlands & 78 & 82 & 80 \\
\hline Norway & 78 & 83 & 81 \\
\hline Poland & 71 & 80 & 76 \\
\hline Portugal & 76 & 83 & 79 \\
\hline Republic of Moldova & 65 & 73 & 69 \\
\hline Romania & 70 & 77 & 73 \\
\hline Russian Federation & 62 & 74 & 68 \\
\hline Slovakia & 71 & 79 & 75 \\
\hline Slovenia & 75 & 82 & 79 \\
\hline Spain & 78 & 84 & 81 \\
\hline Sweden & 79 & 83 & 81 \\
\hline Switzerland & 80 & 84 & 82 \\
\hline
\end{tabular}




\begin{tabular}{lrrr}
\hline \multirow{2}{*}{ Country } & \multicolumn{3}{c}{ Life Expectancy in Years } \\
\cline { 2 - 4 } & Men & Women & Men and Women Combined \\
\hline Tajikistan & 66 & 69 & 67 \\
The Former Yugoslav Republic of Macedonia & 72 & 76 & 74 \\
Turkey & 72 & 77 & 74 \\
Turkmenistan & 60 & 67 & 63 \\
Ukraine & 62 & 74 & 68 \\
United Kingdom & 78 & 82 & 80 \\
Uzbekistan & 66 & 71 & 68
\end{tabular}

Source: WHO, 2010. 


\section{References}

AUH. 1995). Standards zur Expositionsabschätzung. Arbeitsgemeinschaft leitender Medizinalbeamtinnen und -beamten der Länder, Arbeitsausschuss Umwelthygiene $(\mathrm{AUH})$, Bericht des Ausschusses für Umwelthygiene, Behörde für Arbeit, Gesundheit und Soziales (ed), Hamburg.

Brochu P, Ducré-Robitaille J, Brodeur J. 2006. Physiological daily inhalation rates for free-living individuals aged 1 month to 96 years, using data from doubly labeled water measurements: a proposal for air quality criteria, standard calculations and health risk assessment. Human and Ecological Risk Assessment 12:675-701.

Burmaster DE. 1998. Lognormal distributions for skin area as a function of body weight. Risk Analysis 18:27-32.

Calabrese EJ, Stanek EJ, Pekow P, Barnes RM. 1997. Soil ingestion estimates for children residing on a Superfund site. Ecotox Environ Saf 36:358-268.

Commission of the European Communities. 2001. Report from the Commission on Dietary Food Additive Intake in the European Union. Brussels, 01.10.2001, 542 final.

Costeff H. 1966. A simple empirical formula for calculating approximate surface area in children. Arch Dis Child 41:681-683.

D-EPA. 2006. Metoder til fastsættelse af kvalitetskriterier for kemiske stoffer i jord, luft og drikkevand med henblik på at beskytte sundheden. Guidance from the Environmental Protection Agency No. 5, 2006. Danish Ministry of the Environment. In Danish.

Doubilet PM, Benson CB, Nadel AS, Ringer SA. 1997. Improved birth weight table for neonates developed from gestations dated by early ultrasonography. J Ultrasound Med 16:241-249.

DTU-FOOD. 2010. Dietary habits in Denmark 2003-2008. Main results. The National Food Institute, Technical University of Denmark. 2010. www.food.dtu.dk

EC. 2003. Technical Guidance Document on Risk Assessment in Support of Commission Directive 93/67/EEC on Risk Assessment for New Notified Substances, Commission Regulation (EC) No 1488/94 on Risk Assessment for Existing Substances and Directive 98/8/EC of the European Parliament and of the Council Concerning the Placing of Biocidal Products on the Market. http://ecb.jrc.it/tgdoc/

ECB. 2010. The European Union system for the evaluation of substances (EUSES). http://ecb.jrc.it/euses/

ECETOC. 2001. Exposure Factors Sourcebook for European Populations (with Focus on UK Data). Technical Report No. 79. Brussels.

ECHA. 2008a. Guidance on information requirements and chemical safety assessment Chapter R.8: Characterisation of dose [concentration]-response for human health. European Chemicals Agency, 2008 and update 2010.

ECHA. 2008b. Guidance on information requirements and chemical safety assessment. Chapter R.15: Consumer exposure estimation. European Chemicals Agency, 2008 and update 2010.

ECHA. 2010. Guidance on information requirements and chemical safety assessment. European Chemicals Agency,.

http://guidance.echa.europa.eu/docs/guidance_document/information_requireme nts_en.htm?time $=1285273635$

EFSA. 2010. Scientific Opinion on Preparation of a Guidance Document on Pesticide; Exposure Assessment for Workers, Operators, Bystanders and Residents. European Food Safety Authority (EFSA), Parma, Italy. 
EFSA. 2011. DRAFT Guidance on default assumptions used by the EFSA Scientific Panels and Committee, and EFSA Units in the absence of actual measured data. European Food Safety Authority (EFSA), Parma, Italy.

Health and Welfare Canada. 1992. Determination of "toxic" under paragraph 11c) of the Canadian Environmental Protection Act, 1st ed. Ottawa, Ontario, Bureau of Chemical Hazards, Environmental Health Directorate.

Heller K, Sohn W, Burt B, Feigal R. 2000. Water consumption and nursing characteristics of infants by race and ethnicity. J Public Health Dent 60:140-146.

Hopkins SM, Ellis JC. 1980. Drinking water consumption in Great Britain: a survey of drinking habits with special reference to tap-water-based beverages. Technical Report 137, Water Research Centre, Wiltshire Great Britain.

ICRP. 1974. International Commission on Radiological Protection: Report of the Task Group on Reference Man. Oxford, Pergamon Press (ICRP Publication No. 23). Remark: Reference as cited in WHO/IPCS. 1994.

ICRP. 1975. Snyder WS, Cook MJ, Nasset ES, Karhausen LR, Parry Howells G, Tipton IH. 1975. Report of the task group on reference man: a report prepared by a task group of committee 2 of the International Commission on Radiological Protection. ICRP Report No. 23. Pergamon Press, Oxford, UK. Remark: Reference as cited in ECHA. 2008a.

ICRP. 1992. Report of the "Task group on reference man”, International Commission on Radiological Protection, No. 23. Remark: Reference as cited in RIVM. 2006 a.

JRC. 2010. The ExpoFacts Homepage. http://expofacts.jrc.ec.europa.eu/

Kahn H. 2008. Letter from Henry Kahn to Jacqueline Moya, EPA, providing supplemental data to "Estimated daily average per capita water ingestion by child and adult age categories based on USDA's 1994-96 and 1998 continuing survey of food intakes by individuals" (September 18, 2008).

Kahn H, Stralka K. 2008a. Estimated daily average per capita water ingestion by child and adult age categories based on USDA's 1994-96 and 1998 continuing survey of food intakes by individuals. J Exp Sci Environ Epid19:296-404.

Kahn H, Stralka K. 2008b. Estimates of water ingestion for women in pregnant, lactating and non-pregnant and non-lactating child bearing age groups based on USDA's 1994-96, 1998 continuing survey of food intake by individuals. J Human Ecol Risk Assess 14: 1273-1290.

Layton DW. 1993. Metabolically consistent breathing rates for use in dose assessments. Health Phys 64:23-36.

Magré ECC., 2005. Consumer use of Do-it-yourself products - An observational study. RIVM/SIR, Report on a period of work experience. 10936.

RIVM. 2002. Children's Toys Fact Sheet. To assess the risks for the consumer. BA Bilthoven. The National Institute for Public Health and the Environment. RIVM report 612810012/2002. http://www.rivm.nl/bibliotheek/rapporten/ 612810012.pdf

RIVM. 2006a. General Fact Sheet. Limiting conditions and reliability, ventilation, room size, body surface area. Updated version for ConsExpo 4. BA Bilthoven. The National Institute for Public Health and the Environment. RIVM Report 320104002/2006. http://www.rivm.nl/bibliotheek/rapporten/320104002.pdf

RIVM. 2006b. Cosmetics Fact Sheet. To assess the risks for the consumer. Updated version for ConsExpo 4. BA Bilthoven. The National Institute for Public Health and the Environment. RIVM report 320104001/2006. http://www.rivm.nl/ bibliotheek/rapporten/320104001.pdf

RIVM. 2006c. Cleaning Products Fact Sheet. To assess the risks for the consumer. L.C.H. BA Bilthoven. The National Institute for Public Health and the Environment. RIVM report 320104003/2006. http://www.rivm.nl/bibliotheek/ rapporten/320104003.pdf 
RIVM. 2006d. Disinfectant Products Fact Sheet. To assess the risks for the consumer. BA Bilthoven. National Institute for Public Health and the Environment. RIVM report 320005003/2006. http://www.rivm.nl/bibliotheek/rapporten/ 320005003.pdf

RIVM. 2006e. Pest Control Products Fact Sheet. To assess the risks for the consumer. BA Bilthoven. National Institute for Public Health and the Environment. RIVM report 320005002/2006. http://www.rivm.nl/bibliotheek/rapporten/ 320005002.pdf

RIVM. 2007a. Do-It-Yourself Products Fact Sheet. To assess the risks for the consumer. BA Bilthoven. National Institute for Public Health and the Environment. RIVM report 320104007/2007. http://www.rivm.nl/bibliotheek/rapporten/ 320104007.pdf

RIVM. 2007b. Paint Products Fact Sheet. To assess the risks for the consumer. Updated version for ConsExpo 4. BA Bilthoven. The National Institute for Public Health and the Environment. RIVM report 320104008/2007. http://www.rivm.nl/ bibliotheek/rapporten/320104008.pdf

RIVM. 2008. Exposure to chemicals via house dust. BA Bilthoven. The National Institute for Public Health and the Environment. RIVM Report 609021064/2008. http://www.rivm.nl/bibliotheek/rapporten/609021064.pdf

RIVM. 2010. ConsExpo. http://www.rivm.nl/en/healthanddisease/productsafety/ ConsExpo.jsp\#tcm:13-42793

Stanek EJ, Calabrese EJ, Barnes R, Pekow P. 1997. Soil ingestion in adults: results of a second pilot study. Ecotox Environ Saf 36:249-257.

Statistics Netherlands. Participatie vrije tijd. 2005. http://statline.cbs.nl/StatWeb/ .(Centraal Bureau voor de statistiek, www.cbs.nl)

TNsG. 2007. Human Exposure to Biocidal Products; Technical Notes for Guidance (TNsG). June 2007.

http://ecb.jrc.ec.europa.eu/documents/Biocides/TECHNICAL_NOTES_FOR_GUIDA NCE/TNsG_ON_HUMAN_EXPOSURE/TNsG\%20-Human-Exposure-2007.pdf

UK Government Actuary's Department. 1998. The Nations Health, Tables A1 and A2, from the Health and Personal Social Services Statistics information site. www.doh.gov.uk/HPSSS/TBL_A1.HTM and A2.HTM.

UK Office for National Statistics. 1998. Table 7.8 from the Health Survey for England 96, Adult's weight, by age and sex. www.official-documents.co.uk /document/doh/survey96/tab7-8.htm.

UK Office for National Statistics. 1999. Table 3.3 from The Health of Young People '95-97, Weight, by age and sex. www.official-documents.co.uk/ document/doh/survey97/hst3-3.htm.

USDA. 2000. 1994-96, 1998 Continuing Survey of Food Intakes by Individuals (CSFII). CD-ROM. Agricultural Research Service, Beltsville Human Nutrition Research Center, Beltsville, MD. The National Technical Information Service, Springfield, VA; PB2000-500027.

US-EPA. 1985. Development of statistical distributions or ranges of standard factors used in exposure assessments. Washington, DC: Office of Research and Development, Office of Health and Environmental Assessment. EPA 600/8-85-010. NTIS, Springfield, VA. PB85-242667.

US-EPA. 1989. Risk assessment guidance for Superfund. Volume I Human health evaluation manual: Part A. Interim Final. Office of Solid Waste and Emergency Response, Washington, DC. EPA/540-1-89/002.

http://www.epa.gov/oswer/riskassessment/ragsa/index.htm.

US-EPA. 1992. Guidelines for exposure assessment. Washington, DC: U.S. Environmental Protection Agency, Office of Research and Development, Office of Health and Environmental Assessment. EPA/600/Z-92/001. 
US-EPA. 1997. Exposure Factors Handbook. Washington, DC: National Center for Environmental Assessment. Office of Research and Development. http://www.epa.gov/ncea/efh/

US-EPA. 2000. Methodology for Deriving Ambient Water Quality Criteria for the Protection of Human Health (2000). U.S. Environmental Protection Agency, Office of Water, Washington, D.C., EPA-822-00-004. October 2000.

US-EPA. 2002. Estimated Per Capita Fish Intake in the United States. Washington, DC. Office of Water. EPA/821/C-02/003.

US-EPA. 2005. Guidance on selecting age groups for monitoring and assessing childhood exposures to environmental contaminants (2005). Washington, D.C.: U.S. Environmental Protection Agency, EPA/630/P-03/003F.

US-EPA. 2007a. Consolidated Human Activity Database. http://www.epa.gov/ chadnet1/

US-EPA. 2007b. Analysis of fat intake based on the U.S. Department of Agriculture's 1994-96, 1998 Continuing Survey of Food Intakes by Individuals (CSFII). National Center for Environmental Assessment, Washington, DC; EPA/600/R-05/021F. Available from the National Technical Information Service, Springfield, VA. http://www.epa.gov/ncea.

US-EPA. 2008. Child Specific Exposure Factors Handbook 2008. Washington, DC: National Center for Environmental Assessment. Office of Research and Development. http://cfpub.epa.gov/ncea/cfm/recordisplay.cfm?deid=199243

US-EPA. 2009. Exposure Factors Handbook 2009 Update (External Review Draft). Washington, DC: National Center for Environmental Assessment. Office of Research and Development. http://cfpub.epa.gov/ncea/cfm/recordisplay.cfm?deid=209866

US-EPA. 2011. Exposure Factors Handbook 2011 Update (External Review Draft). Washington, DC: National Center for Environmental Assessment. Office of Research and Development. http://www.epa.gov/ncea/efh/pdfs/efh-complete.pdf

US National Center for Health Statistics (NCHS). 2008. National Vital Statistics Reports (NVSR), Deaths: Final Data for 2005, Vol. 56, No. 10, April 24, 2008. http://www.cdc.gov/nchs/data/nvsr/nvsr56/nvsr56_10.pdf.

WHO. 1999a. WHO Global Database on Body Mass Index (BMI).

WHO. 1999b. The World Health Report 1999. Basic Health Indicators, Annex Table 1. http://www-nt.who.int/whosis/basic/ basicqueryprocess.cfm.

WHO. 2008. Guidelines for drinking-water quality. Incorporating $1^{\text {st }}$ and $2^{\text {nd }}$ addenda, Vol.1, Recommendations. - 3rd ed. Geneva: WHO. http://www.who.int/ water_sanitation_health/dwq/fulltext.pdf

WHO. 2010. Part II, Global Health Indicators, Table 1. Mortality and Burden of Disease. World Health Statistics 2010. Geneva: WHO. http://www.who.int/ whosis/whostat/EN_WHS10_Part2.pdf

WHO/IPCS. 1994. Assessing Human Health Risks of Chemicals: Derivation of Guidance Values for Health-based Exposure Limits. Environmental Health Criteria 170. Geneva: WHO. http://www.inchem.org/documents/ehc/ehc/ehc170.htm

WHO/IPCS. 1999. Principles for the Assessment of Risks to Human Health from Exposure to Chemicals. Environmental Health Criteria 210. Geneva: WHO. http://www.inchem.org/documents/ehc/ehc/ehc210.htm

WHO/IPCS. 2000. Human Exposure Assessment. Environmental Health Criteria 214. Geneva: WHO. http://www.inchem.org/documents/ehc/ehc/ehc214.htm

WHO/IPCS. 2001. Glossary of exposure assessment-related terms: a compilation. IPCS Exposure Terminology Subcommittee, International Programme on Chemical Safety, World Health Organization. http://www.who.int/ipcs/publications/ methods/harmonization/en/compilation_nov2001.pdf

WHO/IPCS. 2004. IPCS glossary of key exposure assessment terminology. In: IPCS Risk Assessment Terminology. Harmonization Project Document No. 1. Geneva: WHO. http://www.inchem.org/documents/sids/sids/risk_assess.pdf 
WHO/IPCS. 2005. Principles of Characterizing and Applying Human Exposure Models. IPCS Harmonization Project Document No. 3. Geneva: WHO.

http://whqlibdoc.who.int/publications/2005/9241563117_eng.pdf

WHO/IPCS. 2008. Guidance Document on Characterizing and Communicating Uncertainty in Exposure Assessment. IPCS Harmonization Project Document No. 6. Geneva: WHO. http://www.who.int/ipcs/methods/harmonization/areas/ uncertainty\%20.pdf

WHO/IPCS. 2010. IPCS Harmonization Project. Harmonization of approaches to the assessment of risk from exposure to chemicals website. http://www.who.int/ipcs/ methods/harmonization/en/ 
Nordic Council of Ministers

Ved Stranden 18

DK-1061 Copenhagen K

www.norden.org

\section{Existing Default Values and Recommendations for Exposure Assessment}

Default values are often used in exposure assessments e.g. in modelling because of lack of actually measured data. The quality of the exposure assessment outcome is therefore heavily dependent on the validity and representativeness this input data. Today the used default factors consist of a wide range of more or less well-documented values originating from many different sources.

The purpose of this report is to give an overview and to evaluate exposure factors that are currently used by the authorities and industry in the exposure assessments for both adults (occupational and consumer exposure) and children in relation to REACH. Another important purpose of the report is to contribute towards a further harmonisation of exposure factors by giving recommendations of most valid and representative defaults. These recommendations can be used besides REACH also in biocide's and plant protection product's exposure assessments. The exposure default values were collected from the relevant European sources (ECHA, Consexpo, EUSES, Biocide TNsG, ECETOC, ExpoFacts) as well as from WHO and US-EPA. The following key default factors selected to the evaluation: body weight, body surface area, inhalation rate, soil and dust ingestion, drinking water, food intake, non-dietary ingestion factors, lifetime expectancy, activity factors and consumer products 